\author{
UNIVERSIDADE DE SÃO PAULO \\ ESCOLA DE ENGENHARIA DE SÃO CARLOS
}

Péricles Rafael Pavão Carvalho

\begin{abstract}
Análise numérica bidimensional de sólidos com comportamento visco-elasto-plástico em grandes deformações e situações de contato
\end{abstract}

São Carlos 

Péricles Rafael Pavão Carvalho

\title{
Análise numérica bidimensional de sólidos com comportamento visco-elasto-plástico em grandes deformações e situações de contato
}

\author{
VERSÃO CORRIGIDA \\ (A versão original encontra-se na Escola de Engenharia de São Carlos)
}

Dissertação apresentada ao Departamento de Engenharia de Estruturas da Escola de Engenharia de São Carlos, Universidade de São Paulo, como parte dos quesitos necessários para a obtenção do título de Mestre em Engenharia Civil (Estruturas).

Área de concentração: Estruturas

Orientador: Prof. Dr. Rodolfo André Kuche Sanches

São Carlos 
AUTORIZO A REPRODUÇÃO TOTAL OU PARCIAL DESTE TRABALHO, POR QUALQUER MEIO CONVENCIONAL OU ELETRONNICO, PARA FINS DE ESTUDO E PESQUISA, DESDE QUE CITADA A FONTE.

Ficha catalográfica elaborada pela Biblioteca Prof. Dr. Sérgio Rodrigues Fontes da EESC/USP com os dados inseridos pelo(a) autor(a).

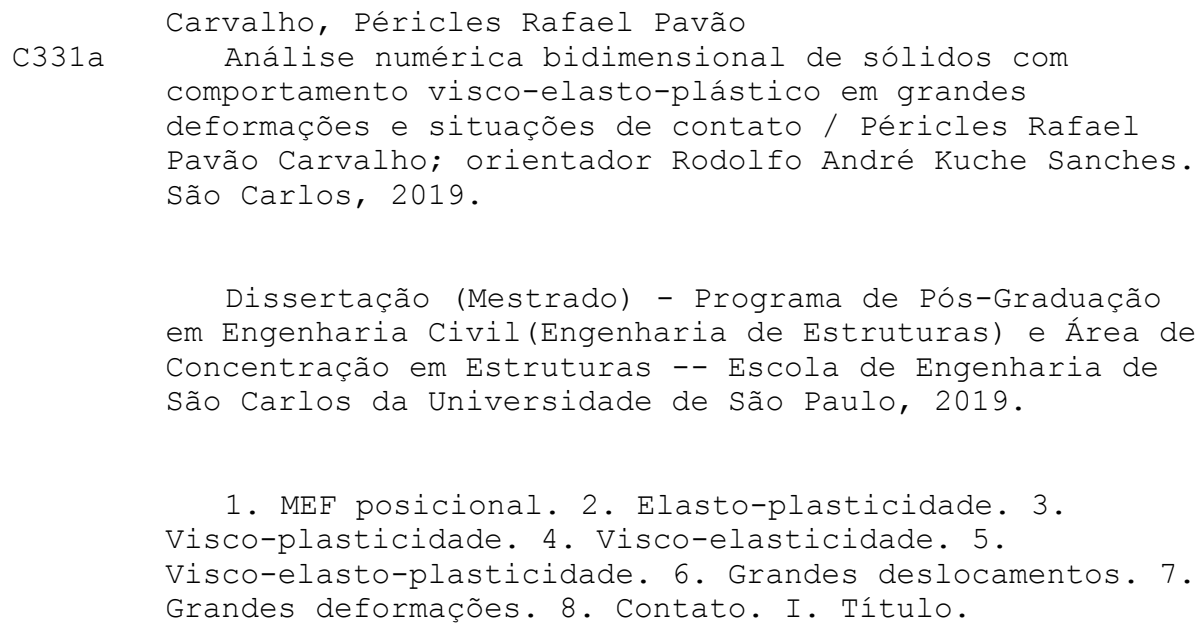

Eduardo Graziosi Silva - CRB - 8/8907 


\section{FOLHA DE JULGAMENTO}

Candidato: Engenheiro PÉRICLES RAFAEL. PAVÃO CARVALHO.

Título da dissertação: "Análise numérica bidimensional de sólidos com comportamento visco-elasto-plástico em grandes deformações e situações de contato".

Data da defesa: 26/03/2019.

Comissão Julgadora:

Resultado:

Prof. Dr. Rodolfo André Kuche Sanches

APROVADO (Orientador)

(Escola de Engenharia de São Carlos/EESC)

Prof. Dr. José Benqque Rubert

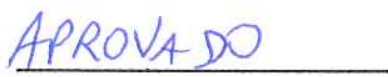

(Universidade Federal de São Carlos/UFSCar)

Prof. Dr. João Paulo Pascon

APROVADO

(Escola de Engenharia de Lorena/EEL-USP)

Coordenador do Programa de Pós-Graduação em Engenharia Civil (Engenharia de Estruturas):

Prof. Titular Humberto Breves Coda

Presidente da Comissão de Pós-Graduação:

Prof. Titular Murilo Araujo Romero 



\section{Agradecimentos}

Aos meus pais, pelo apoio incondicional, e por todo o suporte dado ao longo da minha vida, especialmente nestes últimos dois anos. Vocês são a minha inspiração!

À mulher da minha vida, Wanessa, por ser minha fonte de alegria, e estar ao meu lado em todos os momentos, mesmo no período em que estivemos fisicamente distantes.

Ao professor Rodolfo, pela excelente orientação, e por ter me apresentado a um mundo novo de conhecimento, sem o qual o mestrado seria muito menos interessante.

A todo o corpo de docentes e funcionários do SET, que estiveram sempre de portas abertas para ajudar no que fosse necessário. Em especial ao professor Coda, pelas valiosas contribuições a este trabalho; ao Dorival, pelos excelentes cursos de programação, e pelas inúmeras ajudas e dicas dadas; aos brilhantes professores com quem tive oportunidade de ter aula, em especial Paccola e Proença.

Aos professores do IFMA que me motivaram a seguir a área de estruturas: Rodrigo Neves e André Müller. À minha orientadora da matemática, Vanessa Ribeiro Ramos, pelo seu apoio e paciência.

Aos meus companheiros de sala, Henrique, Caio e Bernardo, pela companhia, pelas conversas, e pelo espírito de cooperação, estando sempre dispostos a parar o que estavam fazendo para ajudar, seja para tentar achar um erro no código ou melhorar algum parágrafo no texto.

Aos demais colegas de turma, em especial Dani, Murilo, Alex, Lucas, Mari e Antônio, que são uns "caminhões carregados de coisas boas".

Aos meus irmãos de orientador, Jeferson e Giovane, que serviram como verdadeiros guias, especialmente no período em que nosso orientador esteve fora do Brasil.

À galera do cafézinho, pelos momentos de descontração e pelas trocas de ideias muitas vezes fundamental para o prosseguimento do trabalho.

A todos que me ajudaram ao longo destes dois anos. 



\section{RESUMO}

\section{CARVAlHO, P. R. P. Análise numérica bidimensional de sólidos com}

comportamento visco-elasto-plástico em grandes deformações e situações de contato. 2019. 177p. Dissertação (Mestrado) - Escola de Engenharia de São Carlos, Universidade de São Paulo, São Carlos, 2019.

Motivado por diversos processos de manufatura, tais como conformação de metais a frio ou mesmo manufatura aditiva, este trabalho consiste no desenvolvimento de um código computacional para a simulação numérica de problemas bidimensionais que abordam três tipos de não-linearidade: a geométrica, presente em situações de grandes deslocamentos; a física, presente no modelo constitutivo do material; e a de contato. Na primeira etapa, desenvolve-se um programa para análise dinâmica bidimensional de sólidos elásticos, utilizando a abordagem posicional do método dos elementos finitos, que engloba naturalmente a não-linearidade geométrica em sua formulação. Em seguida, implementam-se modelos constitutivos não-elásticos para problemas com grandes deformações. No modelo elastoplástico, adota-se o critério de von Mises com encruamento cinemático baseado na lei de Armstrong-Frederick. Essa formulação é então generalizada para o caso visco-plástico, onde é considerado o modelo de Perzyna em conjunto com a lei de Norton. No caso visco-elástico, utiliza-se uma formulação que parte do modelo reológico de Zener. Por fim, apresenta-se um modelo visco-elasto-plástico que consiste no acoplamento dos modelos visco-elástico e visco-plástico descritos anteriormente. Em todos os casos, utiliza-se a decomposição multiplicativa do gradiente da função mudança de configuração. Com respeito à aplicação 2D, consideram-se as hipóteses de estado plano de deformações e estado plano de tensões, onde a última é resolvida numericamente por um procedimento local de Newton-Raphson. Para o problema de contato, aplica-se a estratégia Nó-a-Segmento, sendo as condições de não-penetração impostas com a introdução de multiplicadores de Lagrange. A formulação é testada em cada uma das etapas por meio de exemplos numéricos de verificação. Além disso, para mostrar as potencialidades do código desenvolvido, são propostos diversos exemplos numéricos, sendo alguns inspirados por processos de manufatura existentes. Nesses exemplos, são estudados os efeitos de diferentes parâmetros dos materiais e diferentes taxas de deformação na resposta numérica, permitindo uma análise do comportamento dissipativo decorrente da plastificação e da viscosidade, incluindo a influência desses sobre o amortecimento dinâmico.

Palavras-chave: MEF posicional. Elasto-plasticidade. Visco-plasticidade. Visco-elasticidade Visco-elasto-plasticidade. Grandes deslocamentos. Grandes deformações. Contato. 



\begin{abstract}
CARVALHO, P. R. P. Two-dimensional numerical analysis of solids with visco-elasto-plastic behavior under large strains and contact situations. 2019. 177p. Dissertação (Mestrado) - Escola de Engenharia de São Carlos, Universidade de São Paulo, São Carlos, 2019.

Motivated by several manufacturing processes, such as cold metal forming or even additive manufacturing, in this work we develop a computational code for numerical simulation of two-dimensional problems addressing three types of nonlinearities: geometric nonlinearity, present in large displacements situations; physical non-linearity, present in the material constitutive model; and contact non-linearity. In the first step, we develop a computational program for dynamic analysis of two-dimensional elastic solids using the positional finite element method, which naturally takes into account geometric non-linearity in its formulation. Following, we implement inelastic constitutive models for large strain problems. In the elasto-plastic model, we adopt von Mises yeld criteria and kinematic hardening based on the Armstrong-Frederick law. The formulation is then generalized to the visco-plastic case, where we consider Perzyna model associated with Norton's law. In the visco-elastic case, Zener's rheological model is employed. Finally, we present a visco-elasto-plastic model by coupling the visco-elastic and visco-plastic models described previously. In every case, the multiplicative decomposition of the deformation gradient is employed. Regarding the 2D application, we consider both plane strain and plane stress hypothesis, where the latter is solved numerically by a local Newton-Raphson procedure. For the contact problem, we employ the Node-to-Segment strategy, imposing non-penetration conditions with the introduction of Lagrange multipliers. The resulting computational code is tested in each step by means of numerical verification examples. In addition, to show the potentialities of the developed code, several numerical examples are proposed, some of which inspired by existing manufacturing processes. On these examples, we study the effects of different material parameters and strain rates on the numerical response, allowing an analysis of the dissipative behavior due to plasticity and viscosity, including the influence of these on the dynamic damping.
\end{abstract}

Keywords: Positional FEM. Elasto-plasticity. Visco-plasticity. Visco-elasticity. Viscoelasto-plasticity. Large displacements. Large strains. Contact. 



\section{LISTA DE FIGURAS}

Figura 1 - Mudanças de configuração de um sólido deformável . . . . . . . . . . . 33

Figura 2 - Configurações indeformada e deformada de um cubo infinitesimal . . . 35

Figura 3 - Configurações indeformada e deformada de volume gerado por área infinitesimal . . . . . . . . . . . . . . . . 36

Figura 4 - Corte imaginário na configuração equilibrada de um sólido . . . . . . . 37

Figura 5 - Cubo infinitesimal sujeito a um estado de tensões . . . . . . . . . . . 38

Figura 6 - Gráficos de energia e tensão para uma barra de treliça com modelo SVK 45

Figura 7 - Elementos finitos triangulares em coordenadas adimensionais . . . . . . 49

Figura 8 - Elementos finitos de linha curva em coordenadas adimensionais . . . . 49

Figura 9 - Mapeamento das configurações iniciais e finais . . . . . . . . . . . . 50

Figura 10 - Relação tensão-deformação em modelos elasto-plásticos unidimensionais, onde (I) representa a etapa de carregamento elástico, (II) a etapa a partir do escoamento, e (III) o descarregamento . . . . . . . . . . . 58

Figura 11 - Modelo reológico de material elasto-plástico com encruamento cinemático, adaptado de Vladimirov, Pietryga e Reese (2007) . . . . . . . . . 59

Figura 12 - Superfície de von Mises no espaço das tensões principais considerando encruamento . . . . . . . . . . . . . . . . . 6 61

Figura 13 - Representação dos tipos de encruamento . . . . . . . . . . . . . . 62

Figura 14 - Representação de $\mathbf{N}^{p}$ no espaço das tensões principais . . . . . . . . . . 64

Figura 15 - Esquema de configurações intermediárias do modelo elasto-plástico . 66

Figura 16 - Algoritmo de previsão e correção do modelo elasto-plástico . . . . . . . 74

Figura 17 - Representação do retorno para algoritmos explícitos e implícitos . . . . 75

Figura 18 - Chapa sob tensão uniaxial . . . . . . . . . . . . . . 76

Figura 19 - Diagramas tensão-deformação para o exemplo de tensão uniaxial . . . . 76

Figura 20 - Chapa sob cisalhamento simples . . . . . . . . . . . . . . 77

Figura 21 - Diagramas tensão-deformação para o exemplo de deformação cisalhante simples . . . . . . . . . . . . . . . . . 77

Figura 22 - Evolução dos deslocamentos para cisalhamento simples em material visco-plástico . . . . . . . . . . . . . . . . . . . . . . 81

Figura 23 - Diagramas tensão-deformação para cisalhamento simples em material visco-plástico . . . . . . . . . . . . . . . . . . . . 82

Figura 24 - Evolução do deslocamento para chapa visco-plástica sob tensão uniaxial 82

Figura 25 - Tensões para chapa visco-plástica sob tensão uniaxial . . . . . . . . . . 83

Figura 26 - Deslocamento prescrito em chapa visco-plástica sob cisalhamento simples 84

Figura 27 - Deformação-tensão para chapa visco-plástica sob cisalhamento simples 84

Figura 28 - Tensões de Cauchy ao longo do tempo para chapa visco-plástica sob cisalhamento simples . . . . . . . . . . . . . . . 84 
Figura 29 - Deformações cisalhantes plásticas ao longo do tempo para chapa viscoplástica sob cisalhamento simples . . . . . . . . . . . . . . . 85

Figura 30 - Configurações intermediárias finais para chapa visco-plástica sob cisalhamento simples . . . . . . . . . . . . . . . . . 8 85

Figura 31 - Modelo reológico de Zener, adaptado de Pascon e Coda (2017) . . . . . 88

Figura 32 - Gráficos para o ensaio de fluência em chapa sob tensão uniaxial . . . . 93

Figura 33 - Gráficos para o ensaio de relaxamento em chapa sob tensão uniaxial . . 93

Figura 34 - Evolução de variáveis ao longo do tempo para $\eta=200$. . . . . . . . . 94

Figura 35 - Chapa sob tensão uniaxial com carregamento progressivo . . . . . . . . 95

Figura 36 - Chapa sob tensão uniaxial compressiva com material visco-elástico II . 95

Figura 37 - Gráfico de tempo e deslocamento para o exemplo de cisalhamento simples 96

Figura 38 - Verificações do exemplo de cisalhamento simples em material viscoelástico 96

Figura 39 - Esquema de configurações intermediárias do modelo visco-elasto-plástico 98

Figura 40 - Algoritmo de previsão e correção do modelo visco-elasto-plástico . . . . 103

Figura 41 - Evolução do deslocamento prescrito aplicado em ensaio de relaxamento para chapa com modelo visco-elasto-plástico . . . . . . . . . . . . . 106

Figura 42 - Tensões de Cauchy e alongamentos plásticos para ensaio de relaxamento em chapa com material visco-elasto-plástico . . . . . . . . . . . . . 106

Figura 43 - Alongamentos para ensaio de relaxamento em chapa com material visco-elasto-plástico . . . . . . . . . . . . . . . . . . . . 107

Figura 44 - Evolução da força aplicada em ensaio de fluência para modelo viscoelasto-plástico . . . . . . . . . . . . . . . . . . 107

Figura 45 - Deslocamentos no vértice superior direito da chapa para ensaio de fluência108 Figura 46 - Alongamentos plásticos e viscosos da chapa para ensaio de fluência . . 108 Figura 47 - Evolução dos alongamentos e tensões para ensaio de fluência com $t_{1}=5$ s108

Figura 48 - Deslocamento prescrito em chapa visco-elasto-plástica sob cisalhamento simples . . . . . . . . . . . . . . . . . . 109

Figura 49 - Tensão-deformação para chapa visco-elasto-plástica sob cisalhamento simples . . . . . . . . . . . . . . . . . . . 109

Figura 50 - Tensões de Cauchy ao longo do tempo para chapa visco-elasto-plástica sob cisalhamento simples . . . . . . . . . . . . . . . . . . . . 110

Figura 51 - Deformações cisalhantes plásticas ao longo do tempo para chapa viscoelasto-plástica sob cisalhamento simples . . . . . . . . . . . . . . . . 110

Figura 52 - Tipos de orientações dos elementos finitos de linha curva . . . . . . . . 112

Figura 53 - Projeção normal de ponto projétil sobre segmento alvo . . . . . . . . . 112

Figura 54 - Exemplo do arco deslizante, extraído de Piedade Neto (2013) . . . . . . 116

Figura 55 - Configurações deformadas do arco deslizante e tensão de von Mises . . 116

Figura 56 - Gráficos de deslocamento vertical e tensão horizontal no ponto A . . . 117

Figura 57 - Geometria e malha para exemplo de bloco parcialmente carregado . . . 119

Figura 58 - Diagrama de força-deslocamento para o bloco visco-elástico . . . . . . 120 
Figura 59 - Configurações deformadas na etapa de carga máxima em bloco viscoelástico . . . . . . . . . . . . . . . . . . . 120

Figura 60 - Deslocamento ao longo do tempo para o bloco visco-elástico . . . . . . 121

Figura 61 - Diagrama de força-deslocamento para o caso visco-plástico . . . . . . . 121

Figura 62 - Configurações deformadas para o caso visco-plástico . . . . . . . . . . . 122

Figura 63 - Análise de convergência para jacobianos plásticos e viscosos . . . . . . 122

Figura 64 - Diagrama de força vs. deslocamento para o bloco visco-elasto-plástico . 123

Figura 65 - Deslocamento ao longo do tempo para o bloco visco-elasto-plástico . . 123

Figura 66 - Distribuição de deformação viscosa sobre a configuração deformada em diferentes instantes . . . . . . . . . . . . . . . . . . . . . 124

Figura 67 - Geometria e malha para exemplo dinâmico de viga em balanço . . . . . 124

Figura 68 - Verificação do exemplo dinâmico de viga em balanço com o trabalho de Bathe, Ramm e Wilson (1975) para o caso hiperelástico . . . . . . . . 125

Figura 69 - Comportamento dinâmico de viga visco-elástica para valores extremos

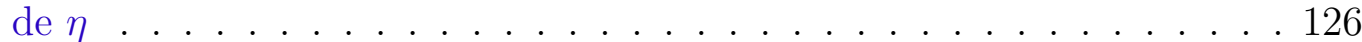

Figura 70 - Comportamento dinâmico de viga visco-elástica para diferentes valores

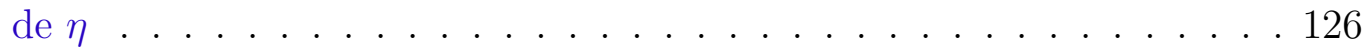

Figura 71 - Gráficos de tempo de estabilização e frequência de viga visco-elástica para diferentes valores de $\eta$, em escala logarítmica na base 2 . . . . . 127

Figura 72 - Comportamento dinâmico de viga visco-plástica para diferentes valores

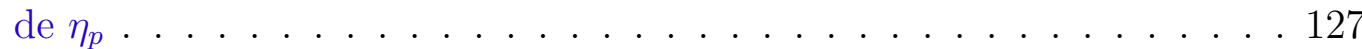

Figura 73 - Comportamento dinâmico de viga visco-elasto-plástica para $\eta_{p}=10^{-3}$ s e diferentes valores de $\eta$

Figura 74 - Gráficos de tempo de estabilização e deslocamento final de viga viscoelasto-plástica para $\eta_{p}=10^{-3} \mathrm{~s}$ diferentes valores de $\eta$, em escala logarítmica na base $2 \ldots \ldots$. . . . . . . . . . . . . . . . . . . . . .

Figura 75 - Resposta de viga visco-elasto-plástica para $\eta=8$ s e diferentes valores de $\eta_{p} \ldots \ldots \ldots \ldots \ldots \ldots \ldots \ldots \ldots \ldots \ldots$

Figura 76 - Configurações finais de viga visco-elasto-plástica para $\eta=8$ s e diferentes valores de $\eta_{p}$, apresentando componentes $\sigma_{11}$ da tensão de Cauchy em mapas de cores . . . . . . . . . . . . . . . . . . . . . 130

Figura 77 - Diagramas de tensão na seção engastada da viga após estabilização para modelo visco-elasto-plástico com $\eta=8$ s e diferentes valores de $\eta_{p} \ldots 130$

Figura 78 - Geometria e malha para exemplo de dobramento simples em chapa metálica . . . . . . . . . . . . . . . . . . . . 130

Figura 79 - Configuração deformada para o exemplo de dobramento simples, com valores de $E_{11}^{p}$ apresentados em mapa de cores . . . . . . . . . . . . . . 131

Figura 80 - Geometria e malha para exemplo de dobramento direcionado em chapa metálica . . . . . . . . . . . . . . . . . . 132

Figura 81 - Configuração deformada para o exemplo de dobramento direcionado, com valores de $E_{11}^{p}$ apresentados em mapa de cores . . . . . . . . . . 132 
Figura 82 - Springback manifestado nos exemplos de dobramento . . . . . . . . . . 132

Figura 83 - Geometria e malha para exemplo de extrusão . . . . . . . . . . . . . 133

Figura 84 - Gráficos de deslocamento para o exemplo de extrusão em material metálico133

Figura 85 - Configurações deformadas para exemplo de extrusão em chapa metálica elasto-plástica, valores de $E_{11}^{p}$ apresentados em mapas de cores . . . . . 134

Figura 86 - Gráficos de deslocamento para o exemplo de extrusão em material polimérico . . . . . . . . . . . . . . . . . . . . . 134

Figura 87 - Configurações deformadas para exemplo de extrusão em chapa polimérica no caso $t_{\max }=2 \mathrm{~s}$, com valores de $E_{11}^{p}$ apresentados em mapas de cores . . . . . . . . . . . . . . . . . . 135

Figura 88 - Geometria e malha para exemplo de impacto entre corpos deformáveis 136

Figura 89 - Gráficos de deslocamento para o exemplo de impacto com modelo hiperelástico . . . . . . . . . . . . . . . . . . . . 137

Figura 90 - Análise de convergência para deslocamentos finais no exemplo de impacto com modelo hiperelástico, em escala logarítmica na base 2 . . . . 137

Figura 91 - Tensão de Cauchy $\left(\sigma_{22}\right)$ no ponto C para impacto com modelo hiperelástico138

Figura 92 - Configurações deformadas de problema de impacto com 112000 passos, mostrando a componente $\sigma_{22}$ das tensões de Cauchy em mapas de cores 138

Figura 93 - Deslocamentos para o exemplo de impacto com modelo visco-elastoplástico . . . . . . . . . . . . . . . . . . . . . . . . 139

Figura 94 - Tensão de Cauchy para o exemplo de impacto com modelo visco-elastoplástico . . . . . . . . . . . . . . . . . . . . . . . 140

Figura 95 - Deformações plásticas para o exemplo de impacto com modelo viscoelasto-plástico . . . . . . . . . . . . . . . . . . . . . . . . . 140

Figura 96 - Configurações deformadas de problema de impacto com material viscoelasto-plástico, apresentando valores de $E_{22}^{p}$ em mapas de cores . . . . . 140

Figura 97 - Geometria e malha para exemplo de abatimento em material altamente viscoso . . . . . . . . . . . . . . . . . . . . . . 141

Figura 98 - Deslocamentos para o exemplo de abatimento . . . . . . . . . . . . . . 142

Figura 99 - Deformações e tensões para o exemplo de abatimento . . . . . . . . . . 142

Figura 100-Configurações deformadas de problema de abatimento, apresentando valores de $\sigma_{22}$ em mapas de cores . . . . . . . . . . . . . . . . . . . 142

Figura 101 - Geometria e malha para exemplo de extrusão dinâmica . . . . . . . . . 143

Figura 102 -Deformações e tensões para o exemplo de abatimento . . . . . . . . . . 143

Figura 103-Deformações para o exemplo de extrusão dinâmica com a Malha 3 . . . 144

Figura 104-Configurações deformadas de problema de extrusão dinâmica com a Malha 3, apresentando valores de $E_{22}^{p}$ em mapas de cores . . . . . . . . 144 


\section{LISTA DE TABELAS}

Tabela 1 - Leis de encruamento isotrópico . . . . . . . . . . . . . 62

Tabela 2 - Parâmetros dos materiais utilizados nos exemplos de validação . . . . 75

Tabela 3 - Parâmetros do material, extraídos de Heeres, Suiker e Borst (2002) . . 81

Tabela 4 - Parâmetros de Perzyna e Norton para o Mild Steel Ck15 . . . . . . . 83

Tabela 5 - Parâmetros de Perzyna e Norton para o material polimérico . . . . . . 83

Tabela 6 - Parâmetros do material, extraídos de Pascon e Coda (2017) . . . . . 92

Tabela 7 - Leis hiperelásticas associadas ao modelo visco-elástico . . . . . . . 94

Tabela 8 - Parâmetros alternativos para material visco-elástico . . . . . . . . 95

Tabela 9 - Parâmetros para material visco-elasto-plástico do tipo polimérico . . . 105

Tabela 10 - Termos da matriz hessiana local de contato usando a técnica Nó-aSegmento e multiplicadores de Lagrange . . . . . . . . . . . . . 114

Tabela 11 - Parâmetros para material visco-elástico do tipo polimérico . . . . . . 120

Tabela 12 - Material visco-elástico para exemplo dinâmico de viga em balanço . . . 125

Tabela 13 - Material visco-plástico para exemplo dinâmico de viga em balanço . . . 127

Tabela 14 - Material visco-elasto-plástico para exemplo dinâmico de viga em balanço128

Tabela 15 - Parâmetros de material visco-elasto-plástico para exemplo de impacto . 139

Tabela 16 - Parâmetros de material visco-elasto-plástico para exemplo de abatimento141 



\section{SUMÁRIO}

INTRODUÇÃO ......................... 21

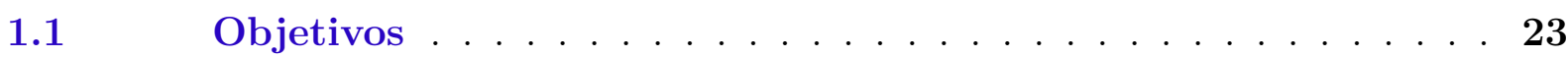

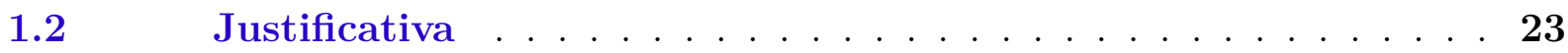

$1.3 \quad$ Estado da arte . . . . . . . . . . . . . . 24

1.3.1 Mecânica dos sólidos computacional . . . . . . . . . . . . 24

1.3.2 Modelos constitutivos . . . . . . . . . . . . . . 25

1.3.2.1 Modelos elasto-plásticos e visco-plásticos . . . . . . . . . . . 26

1.3.2.2 Modelos visco-elásticos . . . . . . . . . . . . . . . . 27

1.3.2.3 Modelos visco-elasto-plásticos . . . . . . . . . . . . . . . . . 29

1.3.3 Modelos numéricos de contato . . . . . . . . . . . . 29

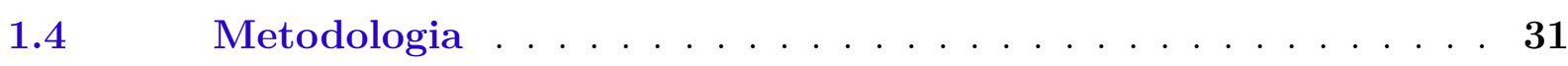

$2 \quad$ MECÂNICA DOS SÓLIDOS . . . . . . . . . 33

2.1 Cinemática dos corpos deformáveis . . . . . . . . . . . 33

2.1.1 Medidas de deformação . . . . . . . . . . . . . . 34

2.1.2 Taxas de deformação . . . . . . . . . . . . . . . . 34

2.1.3 Mudança de volume . . . . . . . . . . . . . . 35

2.1.4 Fórmula de Nanson . . . . . . . . . . . . . . . . . 35

2.1.5 Princípio da conservação da massa . . . . . . . . . . . . . . 36

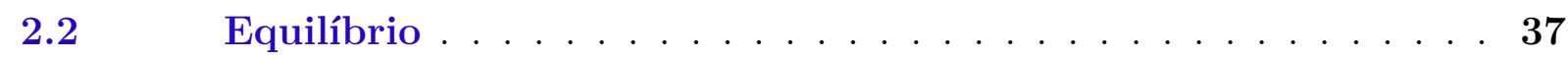

2.2.1 Equilíbrio Euleriano . . . . . . . . . . . . . . 37

$2.2 .2 \quad$ Equilíbrio Lagrangeano . . . . . . . . . . . . . . . . . . 39

2.2.3 Equilíbrio Lagrangeano por abordagem energética . . . . . . . . . . 39

2.2.3.1 Energia cinética . . . . . . . . . . . . . . . . 40

2.2.3.2 Energia potencial das forças externas . . . . . . . . . . . . . 40

2.2.3.3 Energia de deformação . . . . . . . . . . . . . . . 41

2.3 Invariantes dos tensores de tensão e deformação . . . . . . . . . 41

2.4 Tensões hidrostráticas e desviadoras . . . . . . . . . . . 42

2.5 Modelos constitutivos hiperelásticos . . . . . . . . . . . 42

2.5.1 Lei de Saint Venant-Kirchhoff . . . . . . . . . . . . . . . 43

2.5.1.1 Representação Matricial . . . . . . . . . . . . . . . . . . . . . . . . . . . . . . . . . . .

2.5.1.2 Prova da isotropia . . . . . . . . . . . . . . . 44

2.5.1.3 Condições de crescimento . . . . . . . . . . . . . . . . 44

2.5.2 Modelo Neo-Hookeano . . . . . . . . . . . . . . . . . 45

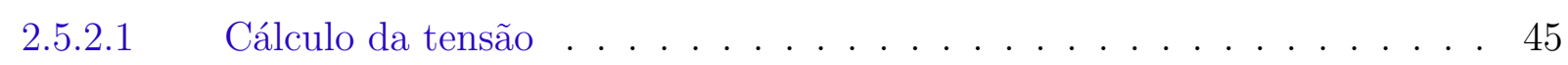

2.5.2.2 Cálculo do operador tangente consistente hiperelástico . . . . . . . . . 46

2.5.3 Estado plano de deformações e de tensões . . . . . . . . . . . . . 46 
$3.2 \quad$ Método de Newton-Raphson . . . . . . . . . . . . 52

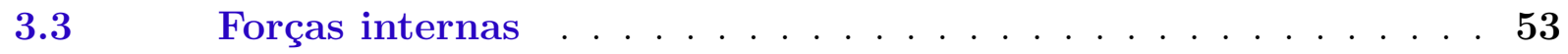

$3.4 \quad$ Forças externas $\ldots \ldots \ldots \ldots \ldots \ldots \ldots \ldots$

$3.5 \quad$ Forças inerciais $\ldots \ldots \ldots \ldots \ldots \ldots \ldots$

$3.6 \quad$ Matriz Hessiana . . . . . . . . . . . . . . . . 55

3.7 Implementação computacional . . . . . . . . . . . 55

4 MODELO ELASTO-PLÁSTICO E VISCO-PLÁSTICO . . . . 57

4.1 Modelo elasto-plástico em pequenas deformações . . . . . . . 57

4.1.1 Concepção unidimensional . . . . . . . . . . . . . . . 58

4.1.2 Decomposição de Green-Naghdi . . . . . . . . . . . . . . . . . 59

4.1 .3 Energia, tensão e dissipação $\ldots \ldots \ldots \ldots \ldots$

4.1.4 Critério de plastificação . . . . . . . . . . . . . . 60

$4.1 .5 \quad$ Encruamento . . . . . . . . . . . . . . . . . 61

4.1.6 Leis de evolução . . . . . . . . . . . . . . . . . . . . . . 62

4.1.7 Condições de complementaridade e consistência . . . . . . . . . . . . 64

$4.2 \quad$ Modelo elasto-plástico em grandes deformações . . . . . . . . 65

4.2 .1 Cinemática dos corpos sob regime elasto-plástico . . . . . . . . . . 65

4.2.1.1 Decomposição de Kröner-Lee . . . . . . . . . . . . . . . . . . . 65

4.2.1.2 Deformações elásticas e plásticas . . . . . . . . . . . . . . 66

4.2.1.3 Taxas de deformação . . . . . . . . . . . . . . . . . 67

$4.2 .2 \quad$ Energia, tensão e dissipação $\ldots \ldots \ldots \ldots$

$4.2 .3 \quad$ Critério de plastificação . . . . . . . . . . . . . . . . . . 69

$4.2 .4 \quad$ Leis de evolução . . . . . . . . . . . . . . . . . . 70

$4.2 .5 \quad$ Operador tangente consistente elasto-plástico . . . . . . . . . . 72

4.2 .6 Implementação computacional . . . . . . . . . . . . . . . . 72

4.2 .7 Exemplos de verificação . . . . . . . . . . . . . . . 75

4.2.7.1 Chapa sob tensão uniaxial . . . . . . . . . . . . . . . 76

4.2.7.2 Chapa sob cisalhamento simples . . . . . . . . . . . . . 77

$4.3 \quad$ Modelo visco-plástico de Perzyna . . . . . . . . . . . . 77

4.3 .1 Leis de evolução . . . . . . . . . . . . . . . . . . 78

4.3.2 Aplicação de algoritmo implícito . . . . . . . . . . . . . . 79

4.3.3 Operador tangente consistente visco-plástico . . . . . . . . . . . . 79

$4.3 .4 \quad$ Exemplos numéricos . . . . . . . . . . . . . . . . 80

4.3.4.1 Chapa sob cisalhamento simples em pequenas deformações . . . . . 80

4.3.4.2 Chapa sob tensão uniaxial . . . . . . . . . . . . . . . . 82

4.3.4.3 Chapa sob cisalhamento simples . . . . . . . . . . . . . . 83

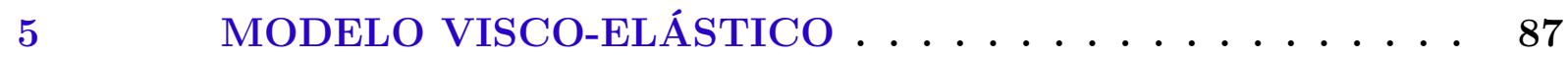

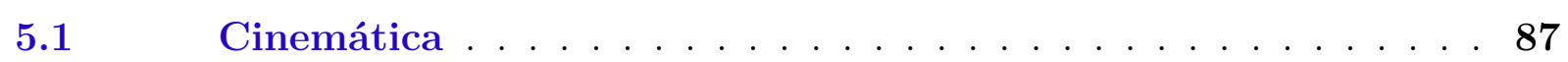


$5.2 \quad$ Energia, tensão e dissipação . . . . . . . . . . . . . . . 88

5.3 Lei de evolução . . . . . . . . . . . . . . . . . . . 90

5.4 Implementação computacional . . . . . . . . . . . . . . . . 91

$5.5 \quad$ Operador tangente consistente visco-elástico . . . . . . . . . . . 91

5.6 Exemplos de verificação . . . . . . . . . . . . . . . 92

5.6.1 Chapa sob tensão uniaxial . . . . . . . . . . . . . . . 92

5.6.1.1 Ensaio de fluência . . . . . . . . . . . . . . . . . . . . 92

5.6.1.2 Ensaio de relaxamento . . . . . . . . . . . . . . 93

5.6.1.3 Carregamento crescente . . . . . . . . . . . . . . . 94

5.6.2 Chapa sob cisalhamento simples . . . . . . . . . . . . . . 96

6 MODELO VISCO-ELASTO-PLÁSTICO . . . . . . . . 97

$6.1 \quad$ Cinemática . . . . . . . . . . . . . . . . 97

6.2 Energia, tensão e dissipação . . . . . . . . . . . . 99

6.3 Critério de plastificação e leis de evolução . . . . . . . . . . . . . 101

6.4 Implementação computacional . . . . . . . . . . . . . . . . . . . 102

6.5 Operador tangente consistente visco-elasto-plástico . . . . . . 104

6.6 Exemplos numéricos . . . . . . . . . . . . . . . . 105

6.6.1 Ensaio de relaxamento . . . . . . . . . . . . . 106

$6.6 .2 \quad$ Ensaio de fluência . . . . . . . . . . . . . . . 107

6.6.3 Chapa sob cisalhamento simples . . . . . . . . . . . . . . 109

7 A ESTRATÉGIA NÓ-A-SEGMENTO PARA PROBLEMAS DE CONTATO ....................... 111

7.1 Detecção do contato . . . . . . . . . . . . . 112

7.2 Imposição das condições de contato . . . . . . . . . . . . . 113

7.2.1 Forças de contato . . . . . . . . . . . . . . . . 113

$7.2 .2 \quad$ Matriz Hessiana . . . . . . . . . . . . . . . . . 114

$7.3 \quad$ Algoritmo . . . . . . . . . . . . . . . . . 114

7.4 Exemplo de verificação: Arco deslizante . . . . . . . . . . . 115

8 EXEMPLOS NUMÉRICOS PROPOSTOS . . . . . . . 119

8.1 Bloco parcialmente carregado . . . . . . . . . . . . . 119

8.1.1 Modelo visco-elástico . . . . . . . . . . . . . . . . 120

8.1.2 Modelo visco-plástico . . . . . . . . . . . . . . . . 121

8.1 .3 Modelo visco-elasto-plástico . . . . . . . . . . . . . . . . . 122

8.2 Análise dinâmica de viga em balanço . . . . . . . . . . . . 124

8.2.1 Modelo visco-elástico . . . . . . . . . . . . . . . . 125

8.2.2 Modelo visco-plástico . . . . . . . . . . . . . . . . . 126

8.2.3 Modelo visco-elasto-plástico . . . . . . . . . . . . . 127

8.3 Dobramento simples de chapa metálica . . . . . . . . . . . . 130

8.4 Dobramento direcionado (Draw Bending) de chapa metálica . 131 


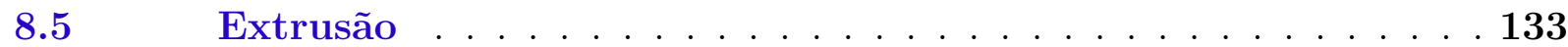

8.5.1 Material metálico com modelo elasto-plástico . . . . . . . . . . . 133

8.5.2 Material polimérico com modelo visco-elasto-plástico . . . . . . . . . . . 134

8.6 Impacto entre corpos deformáveis . . . . . . . . . . . . . . 135

8.6.1 Modelo hiperelástico . . . . . . . . . . . . . 136

8.6.2 Modelo visco-elasto-plástico . . . . . . . . . . . . . . . 138

8.7 Abatimento em material altamente viscoso . . . . . . . . . . . . 141

8.8 Extrusão dinâmica em material altamente viscoso . . . . . . . 143

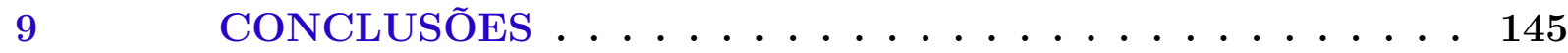

9.1 Modelos constitutivos . . . . . . . . . . . . . . 145

9.2 Exemplos numéricos . . . . . . . . . . . . 146

9.3 Continuidade da pesquisa e sugestões para trabalhos futuros $\quad 147$

REFERENCIAS .................. 149

APÊNDICE A ÁLGEBRA TENSORIAL . . . . . . . . . 161

A.1 Contrações e produtos tensoriais . . . . . . . . . . . 161

A.2 Tensores identidade . . . . . . . . . . . . . 162

A.3 Tensor inverso de quarta ordem . . . . . . . . . . . 162

A.4 Derivadas tensoriais . . . . . . . . . . . . . . . 162

A.5 Relações tensoriais . . . . . . . . . . . . . . . . . 164

APÊNDICE B SOLUÇÃO DOS ALGORITMOS DE RETORNO

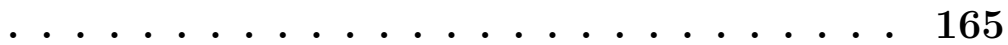

B.1 Modelo elasto-plástico e visco-plástico . . . . . . . . . . . 165

B.2 Modelo visco-elástico . . . . . . . . . . . . . . . . 168

B.3 Modelo visco-elasto-plástico . . . . . . . . . . . . . . 169

B.3.1 Previsão visco-elástica . . . . . . . . . . . . . . . . . . . . . . 169

B.3.2 Correção plástica . . . . . . . . . . . . . . . . . . . . . 169

APÊNDICE C CÁLCULO DO OPERADOR TANGENTE CONSISTENTE ............. 173

C.1 Modelo elasto-plástico e visco-plástico . . . . . . . . . . . 173

C.1.1 Caso não ocorra plastificação . . . . . . . . . . . . . . . . . . . 173

C.1.2 Caso ocorra plastificação . . . . . . . . . . . . . . . . 173

C.2 Modelo visco-elástico . . . . . . . . . . . . . . . . . 174

C.3 Modelo visco-elasto-plástico . . . . . . . . . . . . . 175

C.3.1 Caso não ocorra plastificação . . . . . . . . . . . . . . . . 175

C.3.2 Caso ocorra plastificação . . . . . . . . . . . . . . . . . 176 


\section{Capítulo 1}

\section{Introdução}

As análises estruturais lineares, embora adequadas a muitos problemas de engenharia, ficam restritas a casos em que ocorrem pequenos deslocamentos e pequenas deformações. Para problemas fora desse escopo, tais como os que motivam esse trabalho, faz-se necessária uma análise que leve em consideração efeitos não-lineares, como: não-linearidade geométrica, presente em problemas com grandes deslocamentos, não-linearidade física, presente na maioria dos materiais após atingidos determinados níveis de tensões, e não-linearidade por contato.

Nos problemas com pequenos deslocamentos, a configuração final do corpo pode ser considerada próxima o suficiente da configuração inicial, de forma que o campo de deslocamentos desenvolvido para atingir a configuração de equilíbrio dependa apenas da geometria inicial, das propriedades do material e das forças externas. Já nos casos em que a hipótese de pequenos deslocamentos não é válida, o campo de deslocamentos necessário para que seja atingido o equilíbrio depende também dos próprios deslocamentos, configurando a não-linearidade geométrica.

O efeito de não-linearidade física, por sua vez, ocorre quando não existe proporcionalidade direta ente tensões e deformações, demandando relações constitutivas mais complexas a depender de como o material se comporta durante o carregamento e descarregamento. Um exemplo de modelo constitutivo não-linear é o elasto-plástico, que caracteriza-se por distinguir as deformações ocorridas no sólido entre elásticas, que são reversíveis, e plásticas, que permanecem após o descarregamento. Esse comportamento se deve ao caráter dissipativo do fenômeno, e pode ser observado em diversos materiais de interesse para a indústria, como os metais e polímeros.

Já a não-linearidade de contato resulta da condição de não-penetração entre os corpos, que pode provocar mudanças nas condições de contorno do problema ao longo do tempo.

Esses três tipos de não-linearidades podem ser encontrados, por exemplo, em processos clássicos de manufatura de elementos estruturais, tais como extrusão, conformação, 
laminação e fundição de metais. Outro desenvolvimento mais recente e que vem ganhando cada vez mais espaço é a manufatura aditiva, ou impressão 3D, onde diversos tipos de objetos, incluindo elementos estruturais, são construídos através da deposição por camadas, sendo o processo mais comum o Fused Deposition Modeling, ou FDM (GIBSON; ROSEN; STUCKER, 2014), onde também estão presentes os problemas mencionados acima, além de outros que não pertencem ao escopo deste trabalho, como efeitos termo-mecânicos e mudança de fase.

Nota-se que, com a simulação computacional dos processos de manufatura, é possível fazer previsões sobre as formas e dimensões reais do produto final, magnitude de forças e velocidades de execução necessárias para melhor desempenho e qualidade do produto, além de previsão de tensões residuais resultantes nas peças. Tendo isso como principal motivação, embora as aplicações não se resumam aos processos de manufatura, o presente trabalho consiste no estudo de modelos constitutivos e no desenvolvimento de um código computacional para análise $2 \mathrm{D}$ visco-elasto-plástica de sólidos em situação de contato. Para tal, emprega-se uma formulação do método dos elementos finitos baseada em posições, a qual naturalmente considera os efeitos da não linearidade geométrica e tem se mostrado didaticamente simples.

Com o código computacional desenvolvido foram simulados exemplos como conformação mecânica de metais por meio de dobramento simples e Draw Bending, extrusão de materiais metálicos e poliméricos, problemas de impacto, entre outros. Outra importante contribuição deste trabalho foi o desenvolvimento de um modelo constitutivo visco-elastoplástico para sólidos em grandes deformações, que mostrou resultados satisfatórios e condizentes com o comportamento esperado para esse tipo de material. Deve ser ressaltado que o código desenvolvido ainda não é capaz de simular todos os processos que o motivaram, servindo, no entanto, como base para desenvolvimentos futuros que possam incluir efeitos mais complexos, como, por exemplo, anisotropia e análises termo-mecânicas.

A disposição do texto é a seguinte: neste capítulo são discutidas algumas generalidades do trabalho, objetivos, justificativa, estado da arte e metodologia; no Capítulo 2 é apresentada a base teórica da mecânica dos sólidos, sendo o método numérico utilizado para sua resolução (método dos elementos finitos posicional) abordado no Capítulo 3; nos Capítulos 4, 5 e 6 são descritos os modelos constitutivos utilizados; No Capítulo 7 , discute-se acerca do modelo numérico de contato adotado; finalmente, no Capítulo 8 são apresentados alguns exemplos numéricos simulados com o código desenvolvido, e no Capítulo 9 são feitas as conclusões deste estudo. Além disso, nos Apêndices A, B e C estão alguns tópicos de importância para a escrita ou para o desenvolvimento do trabalho, que, contudo, não caberiam ao longo do texto principal, como conceitos de álgebra tensorial e procedimentos extensos de cálculo. 


\subsection{Objetivos}

O objetivo geral deste trabalho é o desenvolvimento de uma ferramenta computacional, utilizando a abordagem posicional do Método dos Elementos Finitos, para a análise bidimensional do problema não linear de contato/impacto entre sólidos com materiais visco-elásticos, visco-plásticos e visco-elasto-plásticos sujeitos a grandes deslocamentos e grandes deformações. Já os objetivos específicos são:

1. Desenvolvimento de código computacional para análise dinâmica de sólidos com não-linearidades geométricas pelo método dos elementos finitos posicional;

2. Implementação de modelo constitutivo elasto-plástico válido em regime de grandes deformações;

3. Generalização do modelo elasto-plástico para o caso visco-plástico, tomando como base o modelo de Perzyna;

4. Implementação de modelo constitutivo visco-elástico válido em regime de grandes deformações;

5. Desenvolvimento e implementação de modelo constitutivo visco-elasto-plástico por meio de acoplamento entre modelos visco-elástico e visco-plástico;

6. Implementação de modelo numérico de contato no programa desenvolvido, pela estratégia Nó-a-Segmento;

7. Simulação de exemplos numéricos, com análise e verificação dos resultados obtidos.

\subsection{Justificativa}

Com relação à análise de problemas estruturais, sabe-se que abordagens experimentais, apesar de necessárias, demandam um alto custo de materiais e mão-de-obra. Soluções analíticas, por outro lado, quando não impraticáveis, estão restritas a casos muito específicos. Dessa forma, a alternativa numérica se destaca pela sua generalidade e praticidade. Com os recursos computacionais em crescente desenvolvimento, tais análises se tornam viáveis para aplicações cada vez mais complexas, possibilitando um aumento de rapidez e exatidão na solução de problemas das mais diversas áreas da engenharia.

No contexto de materiais visco-elasto-plásticos submetidos a grandes deformações e contato, uma variedade de aplicações podem ser encontradas, por exemplo, em processos de manufatura nas diversas áreas da indústria. Um tipo de material largamente aplicado em tais processos é o metálico, que pode apresentar comportamento visco-elasto-plástico quando submetido a altas temperaturas. Já em processos de conformação a frio, seu comportamento pode ser simplificado por um modelo elasto-plástico ou visco-plástico. Análises numéricas de processos como dobramento, laminação e extrusão podem ajudar a prever o comportamento do Springback, isto é, o recuo elástico sofrido pela peça após o procedimento de conformação. A aplicação de um modelo adequado pode representar até mesmo fenômenos decorrentes de carregamentos cíclicos, aprimorando a qualidade da previsão. A análise de contato nesses casos se justifica pela interação entre o maquinário e a peça sendo manipulada. 
Outro desenvolvimento que vem sendo impulsionado nos últimos anos é a tecnologia de manufatura aditiva, ou impressão 3D. Essa técnica vem revolucionando o mercado pela sua grande versatilidade, sendo utilizada para fabricar desde objetos pequenos até estruturas altamente complexas. Um exemplo de material muito utilizado nesse processo, que apresenta comportamento visco-elasto-plástico, é o polimérico.

Embora ainda poucos dos processos de manufatura que motivaram esse trabalho possam ser simulados realisticamente com o modelo numérico desenvolvido, esse serve como base para futuros trabalhos que possam ampliar o seu leque de aplicações. É importante ainda destacar que, embora a motivação principal seja a modelagem de processos de manufatura, as aplicações deste modelo não se restrigem a isso, uma vez que os efeitos considerados estão presentes nos mais diversos campos da engenharia.

\subsection{Estado da arte}

Para possibilitar o desenvolvimento deste trabalho, torna-se indispensável uma boa base teórica acerca dos fundamentos da mecânica dos sólidos, dos problemas de contato entre corpos deformáveis, e dos modelos constitutivos em grandes deformações, em todos os casos com ênfase no contexto da análise numérica. Tendo isso em vista, apresenta-se nesta seção uma breve revisão bibliográfica desses tópicos, sendo abordados alguns aspectos históricos, principais avanços e referências consultadas.

\subsubsection{Mecânica dos sólidos computacional}

Atualmente, a ferramenta numérica mais difundida não apenas em análises de sólidos e estruturas, como também em diversos âmbitos científicos, é o Método dos Elementos Finitos (MEF). A evolução do método está intimamente relacionada com o advento da computação. Em meados da década de 1950, engenheiros já executavam análises numéricas a partir da discretização do sistema, como pode ser visto em Turner et al. (1956) e Turner, Dill e Martin (1960). Entretanto, de acordo com Bathe (2006), o termo "Método dos elementos finitos" foi cunhado apenas em Clough (1960).

Desde então, o MEF se difundiu amplamente, passando a ser universalmente utilizado e proporcionando resultados satisfatórios nas mais diversas áreas do conhecimento. Entre os trabalhos que contribuíram para o seu desenvolvimento na área da mecânica dos sólidos e das estruturas, podem ser citados, por exemplo: Argyris et al. (1979), Crisfield (1997), Ogden (1997), Bonet e Wood (1997) e Zienkiewicz e Taylor (2005), onde o método é aplicado tanto em casos lineares quanto não-lineares.

Em Coda (2003), é apresentada uma formulação posicional do MEF, assim denominada por utilizar como parâmetros nodais as posições dos nós a partir de um eixo de coordenadas fixo, ao invés dos deslocamentos, como é feito tradicionalmente. Uma formulação semelhante pode ser encontrada em Bonet et al. (2000), aplicada na análise de estruturas pneumáticas de membrana. 
O MEF posicional utiliza uma descrição Lagrangeana total, isto é, toma como referência a posição não-deformada e não-deslocada do corpo em todas as etapas de análise. Sua formulação se destaca por ser didaticamente simples, proporcionando ainda resultados satisfatórios. Além disso, considera intrinsecamente a não-linearidade geométrica, sendo portanto ideal para problemas de grandes deslocamentos. Uma descrição detalhada da formulação pode ser encontrada em Coda (2018).

Vários trabalhos que atestam a confiabilidade do método podem ser citados. Em Coda e Greco (2004), o MEF posicional é aplicado na análise estática de pórticos bidimensionais sob grandes deslocamentos. Já em Greco et al. (2006), utilizou-se elementos de treliças espaciais, em conjunto com um modelo constitutivo elasto-plástico. A análise dinâmica foi introduzida por Greco e Coda (2006) no contexto de pórticos planos, empregando o algoritmo de Newmark para integração no tempo.

Vale ser ressaltado que o algoritmo de Newmark exige conservação da quantidade de movimento, o que é válido no MEF posicional por ser utilizada a descrição Lagrangeana total, que resulta em matriz de massa constante. Uma simples prova dessa passagem pode ser encontrada em Coda e Paccola (2009) e em Sanches e Coda (2013), onde faz-se também considerações sobre a conservação da energia, a qual mostra-se suficiente para os problemas usuais de engenharia quando combina-se MEF posicional e integrador de Newmark.

Em Marques (2006), o método dos elementos finitos posicional é utilizado para resolução de problemas dinâmicos de sólidos bidimensionais elásticos com não-linearidade geométrica, além da consideração de contato com anteparos rígidos. Em Maciel (2008), o método é aplicado na análise de pórticos planos e sólidos tridimensionais.

O método também foi utilizado para análise de elementos de casca, como pode ser visto em Coda e Paccola (2007), Coda e Paccola (2009) e Pascon (2008). Entre outros trabalhos com aplicações dinâmicas, podem ser citados os de Sanches e Coda (2013), Sanches e Coda (2014), Sanches e Coda (2017) e Avancini (2018), onde o método foi aplicado na análise de interação fluido-estrutura. Para aplicações incluindo não-linearidades físicas, podem ser citados os trabalhos de Pascon (2012), Pascon e Coda (2013a), Pascon e Coda (2013b), Pascon e Coda (2015) e Rigobello (2011).

\subsubsection{Modelos constitutivos}

Nesta subseção é feita uma breve revisão bibliográfica acerca de modelos constitutivos elasto-plásticos, visco-plásticos, visco-elásticos e visco-elasto-plásticos. A fim de evitar ambiguidades, é importante uma breve discussão acerca da nomenclatura utilizada neste trabalho: a denominação "elasto-plástico" refere-se tradicionalmente a modelos que apresentam elasticidade e plasticidade independentes de taxas. Já modelos visco-plásticos, também denominados elasto-viscoplásticos na literatura, apresentam elasticidade independente de taxa e comportamento viscoso (dependência temporal) associado à plasticidade. Modelos 
visco-elásticos, por sua vez, apresentam viscosidade associada ao comportamento elástico do material. Por fim, modelos visco-elasto-plásticos, também denominados viscoelásticoviscoplásticos na literatura, apresentam viscosidade associada tanto ao comportamento elástico quanto ao comportamento plástico do material.

\subsubsection{Modelos elasto-plásticos e visco-plásticos}

De acordo com Hill (1998), o estudo científico da plasticidade teve início por volta de 1864, quando Tresca estabeleceu experimentalmente que materiais metálicos sofrem escoamento a partir de um valor crítico de tensão cisalhante. Desde então, diversos pesquisadores contribuíram significativamente para desenvolver essa linha de pesquisa, como Saint-Venant, Levy, von Mises, Hencky, Prandtl e Taylor (KHAN; HUANG, 1995).

Entre os trabalhos que abordam a teoria clássica da plasticidade em pequenas deformações, podem ser citados os de Johnson e Mellor (1983), Chakrabarty (1987), Khan e Huang (1995), Crisfield (1997) e Hill (1998). Nos trabalhos de Coda e Paccola (2014) e Coda, Sampaio e Paccola (2015), modelos elasto-plásticos são aplicados em conjunto com o método dos elementos finitos posicional, sendo adotada a decomposição de Green-Naghdi, em que o tensor deformação de Green-Lagrange é expresso pela soma de suas parcelas elástica e plástica. No entanto, conforme discutido por Eterovic e Bathe (1991), essa decomposição apresenta incoerências que limitam seu uso tradicionalmente ao regime de pequenas deformações.

Para o caso de grandes deformações, podem ser consultados os trabalhos de Simo (1992), Khan e Huang (1995) e Simo e Hughes (2000). No contexto do departamento de estruturas, destacam-se os trabalhos de Rubert (1997) e Pascon (2012). Em grande parte das formulações, utiliza-se a bem aceita decomposição de Kröner-Lee (KRÖNER, 1960; LEE, 1969; HAUPT, 1985), onde o gradiente da função mudança de configuração é decomposto de forma multiplicativa entre suas parcelas elástica e plástica. Quanto à lei constitutiva, a representação pode ser feita por meio de uma abordagem termodinâmica, isto é, partindo da energia de dissipação (PASCON; CODA, 2013a). Uma das maneiras de representar tal dissipação é pela inequação de Clausius-Duhem (HOLZAPFEL, 2000), que resulta, para abordagens Lagrangeanas, em uma relação de conjugação termodinâmica entre as taxas de deformação plástica e o denominado tensor de Mandel (MANDEL, 1973). Entre os trabalhos que utilizam esse conceito, podem ser citados os de Svendsen (1998), Svendsen et al. (1998), Dettmer e Reese (2004), Pascon e Coda (2013a), Pascon e Coda (2013b). Tal abordagem pode ser vista também em modelos que incluem anisotropia, como o apresentado em Vladimirov, Pietryga e Reese (2010).

Dois fenômenos importantes que são observados nos problemas de elasto-plasticidade são os chamados efeitos de Bauschinger e Ratcheting (CHABOCHE, 1986; MOLLICA; RAJAGOPAL; SRINIVASA, 2001; OLIVEIRA et al., 2007). O primeiro está relacionado ao fato das deformações em uma direção influenciarem na tensão de escoamento da direção 
oposta, enquanto o segundo está relacionado ao aumento da deformação média devido à aplicação de tensões cíclicas (VLADIMIROV; PIETRYGA; REESE, 2007). Nesse sentido, a lei de encruamento cinemático de Armstrong-Frederick (ARMSTRONG; FREDERICK, 1966) é amplamente utilizada, porém, apresenta limitações fora do contexto de pequenas deformações, uma vez que a objetividade da lei de evolução é comprometida.

Estudos para extensão da lei de Armstrong-Frederick ao contexto de grandes deformações foram realizados por Dettmer e Reese (2004) e Vladimirov, Pietryga e Reese (2007), onde foi utilizada uma estratégia denominada "tipo Chaboche", que parte da utilização de taxas temporais objetivas, como a derivada de Jaumann (DOGUI; SIDOROFF, 1985; PASCON; CODA, 2013a), para escrever a lei de evolução. Nesse caso utiliza-se um tensor denominado tensão de retorno (Back Stress) como variável interna representando o encruamento cinemático. Já nos trabalhos de Vladimirov, Pietryga e Reese (2010) e Brepols, Vladimirov e Reese (2014) é utilizada uma estratégia que parte da decomposição das deformações plásticas entre parcelas que representam diferentes efeitos na estrutura cristalina (LION, 2000). A lei de evolução resultante nesse caso é naturalmente objetiva, dispensando a utilização de taxas temporais alternativas.

Para o caso visco-plástico, alguns modelos clássicos podem ser mencionados, como o de Perzyna (PERZYNA, 1966) e o de Duvaut-Lions (DUVAUT; LIONS, 1976), esse último diferenciando-se por definir múltiplas superfícies de plastificação para o domínio elástico (SIMO; HUGHES, 2000). Uma generalização desses modelos ao caso de grandes deformações é feita no trabalho de Ibrahimbegović e Chorfi (2000), por meio da decomposição multiplicativa e abordagem termodinâmica, conforme mencionado previamente, porém, utilizando uma descrição espacial (Euleriana). Já no trabalho de Mähler, Ekh e Runesson (2001), é utilizada uma descrição Lagrangeana, sendo adotado um formato generalizado do modelo de Perzyna, considerando o conceito de admissibilidade visco-plástica. Mais aplicações do modelo de Perzyna no regime de grandes deformações podem ser vistas, por exemplo, nos trabalhos de Ponthot (2002), Garino et al. (2013) e Careglio et al. (2016). É importante ser dito que tal modelo caracteriza-se por permitir um descumprimento (controlado) do critério de plastificação. Como alternativa a isso, Wang, Sluys e Borst (1997) propuseram um modelo visco-plástico denominado "consistente", que introduz a dependência temporal no próprio critério de plastificação. Um estudo mostrando uma comparação entre os dois modelos pode ser visto em Heeres, Suiker e Borst (2002). Uma revisão mais completa sobre modelos visco-plásticos pode ser encontrada, por exemplo, em Chaboche (2008).

\subsubsection{Modelos visco-elásticos}

De acordo com Christensen (2013), o avanço no estudo de modelos constitutivos visco-elásticos se deve principalmente à produção e utilização em larga escala de materiais poliméricos. Para simular esse comportamento, diversos modelos reológicos foram desenvolvidos, como, por exemplo, o de Kelvin-Voigt, que pode ser representado pelo arranjo 
em paralelo de uma mola (comportamento elástico) com um amortecedor (comportamento viscoso), e o de Maxwell, representado pelo arranjo em série entre os mesmos (LEMAITRE; CHABOCHE, 1985). A partir desses, derivam os denominados modelos de três parâmetros, discutidos, por exemplo, em Findley, Lai e Onaran (1989) e Huber e Tsakmakis (2000). Dentre esses, os mais comuns são os de Boltzmann e Zener, representados, respectivamente, pelo arranjo em série de uma mola com o modelo de Kelvin-Voigt e pelo arranjo em paralelo de uma mola com o modelo de Maxwell. Em Huber e Tsakmakis (2000), é demonstrado que esses modelos apresentam propriedades mecânicas idênticas para o caso de pequenas deformações, desde que feita a devida associação de seus parâmetros. Já no caso de deformações finitas, os modelos se diferenciam essencialmente por efeitos de segunda ordem, conforme mostrado pelo mesmo autor.

Uma das formas mais comuns de tratar a viscosidade é pelo modelo de integral de convolução (CIM), que baseia-se no conceito de funções de relaxação (SIMO, 1987; LEMAITRE; CHABOCHE, 1985; HOLZAPFEL, 1996; LEMAITRE, 2001). Nesse caso, o comportamento constitutivo é descrito por meio de integrais hereditárias e, portanto, sua aplicação requer o conhecimento de todo o histórico de deformação do material.

Dessa forma, tornam-se vantajosas as formulações que se baseiam no conceito de variáveis internas (IVM), iniciadas com o trabalho de Green e Tobolsky (1946), e amplamente utilizadas até os dias de hoje (ver, por exemplo, Holzapfel e Simo (1996) e Lion (1997)). Nesse contexto, a viscosidade é tratada por meio de leis de evolução em forma diferencial, que podem ser resolvidas numericamente por integradores temporais. Uma comparação entre as abordagens CIM e IVM pode ser vista no trabalho de Petiteau et al. (2013), onde são destacadas as vantagens e desvantagens dos métodos para cada tipo de situação.

Aproveitando a ideia introduzida por Kröner (1960) e Lee (1969) no contexto da elasto-plasticidade, diversos autores começaram a utilizar o conceito de variável interna por meio da decomposição das deformações entre parcelas elásticas e viscosas. Isso pode ser visto, por exemplo, nos trabalhos de Sidoroff (1974) e Lubliner (1985), onde foi utilizada a decomposição multiplicativa do gradiente da função mudança de configuração. Tal decomposição também foi aplicada nos trabalhos de Tallec, Rahier e Kaiss (1993), Reese e Govindjee (1997), Huber e Tsakmakis (2000), Hasanpour, Ziaei-Rad e Mahzoon (2009), Petiteau et al. (2013) e Pascon e Coda (2017), em conjunto com formulações termodinâmicas. Estratégias diferenciais para tratar a viscosidade podem ser vistas também nos trabalhos de Mesquita e Coda (2002), Mesquita e Coda (2003), Mesquita e Coda (2007) e Madeira e Coda (2016) para formulações baseadas no modelo reológico de KelvinVoigt. Com relação à aplicações de modelos visco-elásticos em análises bidimensionais considerando estado plano de tensões, os trabalhos de Kroon (2011) e Pascon (2018) podem ser citados. 


\subsubsection{Modelos visco-elasto-plásticos}

Embora os estudos de visco-elasticidade e visco-plasticidade já tenham sido bem consolidados na literatura, ainda são relativamente escassos os modelos que consideram um acoplamento de ambos. Materiais como os polímeros, por exemplo, apresentam dependência temporal tanto na etapa após a plastificação quanto na anterior. No trabalho de Lammens et al. (2017) podem ser vistas as curvas experimentais demonstrando tal comportamento no material Poliamida 12, muito utilizado em processos de manufatura aditiva (impressão 3D), sendo também observados efeitos anisotrópicos. Outros resultados experimentais podem ser vistos em Lai e Bakker (1995) para o polietileno de alta densidade (PEAD).

No contexto de pequenas deformações, alguns modelos desenvolvidos para os materiais poliméricos podem ser citados, como os de Kim e Muliana (2009) e Miled, Doghri e Delannay (2011). No primeiro, foi utilizada uma abordagem integral para a parcela viscoelástica, enquanto no segundo uma abordagem diferencial, sendo em seguida generalizado para o caso de grandes deformações nos trabalhos de Nguyen et al. (2016) e Gudimetla e Doghri (2017). Esse último se difere do primeiro pelo fato de que foi utilizada uma formulação termodinâmica. Ambos, no entanto, fazem uso da decomposição multiplicativa entre as parcelas visco-elásticas e visco-plásticas do gradiente da função mudança de configuração. Outros modelos para materiais poliméricos em grandes deformações são apresentados em Holmes, Loughran e Suehrcke (2006) e Abdul-Hameed et al. (2014). O comportamento visco-elasto-plástico também é estudado em materiais do tipo asfáltico, como pode ser visto nos trabalhos de Drescher, Kringos e Scarpas (2010) e Darabi et al. (2011), materiais com self-healing (SHAHSAVARI et al., 2016), e até mesmo em materiais metálicos sobre altas temperaturas (BENAARBIA; ROUSE; SUN, 2018).

\subsubsection{Modelos numéricos de contato}

Os primeiros estudos sobre contato remontam à 1881, tendo sido conduzidos pelo físico alemão Heinrich Hertz, motivado por experimentos de interferência ótica em lentes de vidro (JOHNSON, 1987). Entre os trabalhos que moldaram o conhecimento nessa linha de pesquisa, valem ser citados os de Hughes et al. (1976), Hallquist, Goudreau e Benson (1985), Bathe e Chaudhary (1985), Chaudhary e Bathe (1986) e Benson e Hallquist (1990).

Do ponto de vista numérico, a modelagem do contato entre corpos deformáveis pode ser dividida em dois sub-problemas: a detecção da intersecção e a imposição das condições de não-penetração. Para ambos, a técnica de discretização adotada influencia diretamente no procedimento da solução.

Em problemas onde se pode garantir a coincidência das malhas na região do contato, uma das formulações mais simples é a Nó-a-Nó (WRIGGERS, 2006), na qual o modelo é definido por pares de nós devidamente alinhados. No entanto, para o caso geral, a estratégia mais comumente adotada é a do tipo Nó-a-Segmento, introduzida em Hughes et al. (1976) e Hallquist (1979), onde uma das interfaces de contato é discretizada por 
elementos nodais, denominados nós projéteis, enquanto a segunda interface é discretizada por elementos de linhas curvas (no caso 2D), denominados segmentos alvos. Explicações detalhadas sobre a formulação podem ser encontradas em Wriggers (2006), Piedade Neto (2009) e Yastrebov (2013). Outros exemplos de trabalhos que utilizam essa abordagem são Bathe e Chaudhary (1985), Hallquist, Goudreau e Benson (1985), Wriggers e Simo (1985), Simo, Wriggers e Taylor (1985), Wriggers, Van e Stein (1990), Papadopoulos e Taylor (1992) e Zhong (1993).

Conforme apontado por Zavarise e Lorenzis (2009), alguns problemas podem ser encontrados em casos especiais do algoritmo Nó-a-Segmento, mais especificamente na etapa de detecção, devido à descontinuidade do vetor normal entre segmentos vizinhos. Embora o uso de elementos de ordem superior diminua a frequência de tais problemas, o trabalho previamente citado menciona a necessidade de cuidados especiais para evitar resultados inconsistentes ou problemas de convergência.

Outra desvantagem do método Nó-a-Segmento é que apenas os nós projéteis são controlados, não sendo detectados os casos em que os nós da interface alvo penetrem os segmentos do sólido oposto. Em outras palavras, o método é eficaz em detectar o contato e aplicar restrições nos nós, mas não é capaz de generalizar a restrição para toda a superfície de contorno. Em determinadas situações essa desvantagem pode proporcionar erros consideráveis, sendo uma das soluções a aplicação dos chamados métodos de dois passes, conforme discutido em Puso e Laursen (2004). Outra implicação desse fato é que a superfície de contato deve ter a discretização bem refinada para que sejam proporcionados bons resultados.

Outro método atualmente muito difundido para modelagem de contato é o Mortar, que teve início com o trabalho de Bernardi, Debit e Maday (1990) e foi aperfeiçoado em Bernardi, Maday e Patera (1994), apenas como uma formulação matemática que proporciona uma técnica de compatibilização de domínios. O método começou a ser utilizado em problemas de contato com o trabalho de Belgacem, Hild e Laborde (1998). Essa técnica, assim como o Nó-a-Segmento, fornece uma alternativa para simular contato entre malhas não-coincidentes, porém baseia-se em uma abordagem Segmento-a-Segmento, como pode ser visto, por exemplo, em Puso e Laursen (2004).

No método Mortar, todos os elementos de contorno passíveis de contato são discretizados como elementos de linha curva (segmentos) no caso 2D, ou de superfície no caso 3D, e o contato é determinado por integração numérica ao longo do domínio da interface. Dessa forma, tanto a deteç̧ão quanto a restrição são realizadas nos pontos de integração. Uma de suas vantagens é garantir a não-penetrabilidade nas regiões entre os nós, permitindo portanto uma discretização menos refinada na região de contato quando comparado com a abordagem Nó-a-Segmento. Entre outros trabalhos que utilizam o método, podem ser citados Fischer e Wriggers (2005), Yang, Laursen e Meng (2005), Fischer e Wriggers (2006), Hartmann e Ramm (2008), Popp, Gee e Wall (2009), Tur et al. 
(2012), Piedade Neto (2009), Piedade Neto (2013).

Para o caso de domínios particionados, uma técnica comumente adotada baseia-se na troca de condições de contorno Dirichlet-Neumann. Essa é especialmente utilizada no contexto de interação fluido-estrutura (BAZILEVS; TAKIZAWA; TEZDUYAR, 2013; FERNANDES; CODA; SANCHES, 2018), onde os domínios são naturalmente particionados por apresentarem diferentes comportamentos físicos, mas o método também pode ser aplicado em contato entre sólidos (WRIGGERS, 2006).

Também valem ser mencionados outros tipos de abordagem baseados em áreas de influência, como a técnica de "território" apresentada em Zhong e Nilsson (1996), e a de "pinball”, apresentada em Belytschko e Neal (1991) e Belytschko e Yeh (1993).

Quanto à aplicação de modelos de contato em análise dinâmica, outros desafios surgem, uma vez que a forte não-linearidade do problema provoca mudanças repentinas nos valores de aceleração e velocidade, que podem levar à instabilidade da solução quando utilizado o algoritmo de Newmark com os parâmetros tradicionais, como atestado no trabalho de Chaudhary e Bathe (1986). Técnicas para resolver essa inconformidade podem ser vistas, por exemplo, em Carpenter, Taylor e Katona (1991), Taylor e Papadopoulos (1993), Solberg e Papadopoulos (1998) e Hu (1997). O último propõe novos parâmetros para o algoritmo de Newmark que levam a resultados estáveis e precisos mesmo para problemas de impacto com altas frequência. No entanto, esta alteração provoca um amortecimento numérico altamente sensível à discretização temporal adotada, sendo, portanto, necessário que sejam utilizados intervalos de tempo suficientemente pequenos para diminuir os erros provocados. Entre outros trabalhos que confirmam a eficiência desse estudo, podem ser citados os de Greco (2004), Marques (2006) e Minski (2008).

\subsection{Metodologia}

Para fins de organização, o desenvolvimento do trabalho é dado em três etapas principais, em cada qual são desenvolvidas respectivamente a não-linearidade geométrica, a não-linearidade física e a não-linearidade por contato.

Na primeira etapa, desenvolve-se um programa computacional para análise dinâmica de sólidos sob grandes deslocamentos utilizando o Método dos Elementos Finitos posicional, partindo de uma abordagem energética para obtenção das equações de equilíbrio e adotandose, inicialmente, a lei constitutiva de Saint Venant-Kirchhoff. Utilizam-se os métodos de Newton-Raphson e Newmark- $\beta$ para resolução do sistema não-linear e integração temporal, respectivamente. $\mathrm{O}$ código é escrito em linguagem Fortran, em conjunto com a ferramenta OpenMP (DAGUM; MENON, 1998) para multi-processamento. O sistema linear resultante em cada iteração é resolvido pela rotina HSL MA67 (HSL, 2013).

Na segunda etapa, são implementados modelos constitutivos visco-plástico, viscoelástico e visco-elasto-plástico para regimes de grandes deformações no código desenvolvido, todos baseados em abordagens termodinâmicas e utilizando como estratégia a decomposição 
multiplicativa do gradiente da função mudança de configuração. O modelo elasto-plástico é baseado nos trabalhos de Svendsen (1998), Dettmer e Reese (2004) e Pascon e Coda (2013a), com encruamento cinemático de Armstrong-Frederick seguindo a forma descrita no trabalho de Vladimirov, Pietryga e Reese (2010). A generalização para o caso visco-plástico é feita por meio do modelo de Perzyna em conjunto com a lei de Norton. Para o modelo visco-elástico, segue-se o trabalho de Pascon e Coda (2017), onde é utilizado o modelo reológico de Zener. Por fim, desenvolve-se um modelo visco-elasto-plástico por meio do acoplamento dos modelos visco-elástico e visco-plástico descritos. Tal acoplamento é feito por meio da substituição da parcela elástica do modelo visco-plástico por uma parcela visco-elástica. Para todos os casos, no processo de evolução da lei constitutiva emprega-se método implícito de Euler para integração temporal.

Em seguida, é implementado um modelo numérico de contato utilizando a abordagem Nó-a-Segmento. Para a deteç̧ão do contato, emprega-se a técnica do ponto de mínima distância, sendo aplicado um procedimento de Newton-Raphson local para a resolução do sistema não linear que define as coordenadas de tal ponto. Quanto à imposição das condições de contato, adota-se o método dos multiplicadores de Lagrange.

Em cada estágio de implementação, verifica-se o código através do estudo de modelos simples, e ao final, o programa desenvolvido é aplicado para diversas simulações numéricas a fim de tornar claro o comportamento dos modelos constitutivos e do modelo numérico frente a diferentes discretizações espaciais e temporais. Para a geração de malhas, visualização de pós-processamento e geração de gráficos, são utilizados os softwares OpenSource Gmsh (GEUZAINE; REMACLE, 2009), ParaView (AYACHIT, 2015) e Gnuplot (WILLIAMS; KELLEY; many others, 2013), respectivamente. 


\section{Capítulo 2}

\section{Mecânica dos sólidos}

A mecânica dos sólidos possui duas formas principais de descrição matemática: Lagrangeana (ou referencial) e Euleriana (ou espacial). Na primeira, toma-se como referência a configuração inicial ou indeformada do corpo, enquanto na segunda utiliza-se a configuração final ou deformada. Neste capítulo, é dada ênfase à descrição Lagrangeana, empregada no presente trabalho. Maiores detalhes sobre os temas abordados aqui podem ser encontrados em Ogden (1997), Bonet e Wood (1997), Holzapfel (2000) e Coda (2018).

\subsection{Cinemática dos corpos deformáveis}

Seja um corpo com configuração inicial ou indeformada $\Omega^{0}$, e configuração atual $\Omega^{1}$. É de interesse da cinemática dos sólidos deformáveis descrever as mudanças de posições e forma às quais tal corpo foi submetido. Para tal, define-se a função mudança de configuração $\boldsymbol{\zeta}$ como sendo uma aplicação vetorial que mapeia as posições atuais (denotadas por $\mathbf{y}$ ) a partir das iniciais (denotadas por $\mathbf{x}$ ), conforme ilustra a Figura 1. Além disso, denota-se por $\mathbf{A}$ o gradiente da função mudança de configuração, isto é:

$$
\mathbf{A}=\nabla_{\mathbf{x}}(\boldsymbol{\zeta}) \quad \text { ou } \quad A_{i j}=\zeta_{i, j}=\frac{\partial \zeta_{i}}{\partial x_{j}}
$$

Figura 1 - Mudanças de configuração de um sólido deformável

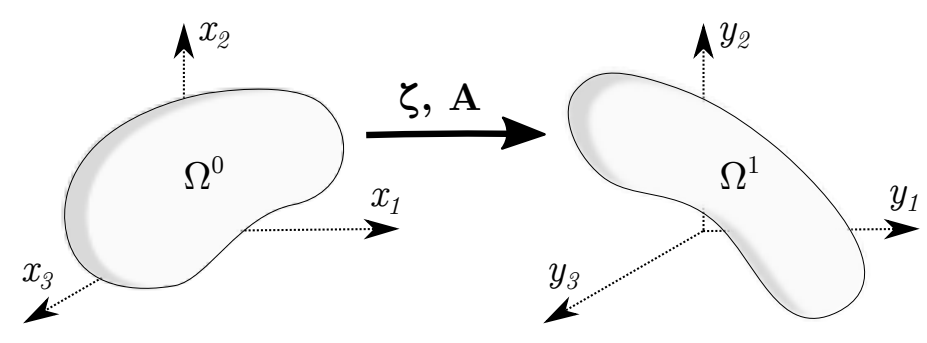

Fonte: Elaborada pelo autor ${ }^{1}$

Um resultado fundamental da mecânica do contínuo é o teorema da decomposição

1 Uma vez que todas as figuras e tabelas deste trabalho são elaboradas pelo autor, optou-se por omitir a fonte nos casos posteriores, sendo informado na legenda caso essa tenha sido adaptada de outro trabalho 
polar (BONET; WOOD, 1997), que afirma que A pode ser unicamente decomposto nas formas

$$
\mathbf{A}=\mathbf{R U}=\mathbf{v R},
$$

chamadas, respectivamente, decomposição polar à direita e decomposição polar à esquerda. O tensor $\mathbf{R}$ é denominado tensor rotação, pois representa apenas os movimentos de corpo rígido sofridos pelo corpo, isto é, movimentos de rotação sem que haja mudança de forma propriamente dita. Por consequência, $\operatorname{det}(\mathbf{R})=1$. Outra importante propriedade de $\mathbf{R}$ é a sua ortogonalidade, isto é, $\mathbf{R}^{T} \mathbf{R}=\mathbf{I}$, onde $\mathbf{I}$ é a identidade.

Já os tensores $\mathbf{U}$ e $\mathbf{v}$, chamados respectivamente de alongamento à direita (ou material) e alongamento à esquerda (ou espacial), representam a parcela de deformação pura de $\mathbf{A}$, e são simétricos, ou seja, $\mathbf{U}=\mathbf{U}^{T}$ e $\mathbf{v}=\mathbf{v}^{T}$.

\subsubsection{Medidas de deformação}

Uma medida de deformação é uma grandeza que permite mensurar pontualmente a mudança de forma ocorrida entre as configurações inicial e atual. Essa deve ser objetiva, isto é, independente de movimentos de corpo rígido ou da escolha de eixos adotada. Com isso em vista, define-se o alongamento à direita de Cauchy-Green:

$$
\mathbf{C}=\mathbf{A}^{T} \mathbf{A} \text {. }
$$

Da decomposição polar à direita, segue que

$$
\mathbf{C}=(\mathbf{R U})^{T}(\mathbf{R U})=\mathbf{U}^{T} \mathbf{R}^{T} \mathbf{R U}=\mathbf{U I U}=\mathbf{U U}=\mathbf{U}^{2},
$$

ou seja, $\mathbf{C}$ depende apenas das parcelas de deformações puras de $\mathbf{A}$, provando a sua objetividade. Outra consequência de (2.4) é o fato de que fenômenos como movimentos de corpo rígido, que não provocam deformações, resultam em $\mathbf{C}=\mathbf{I}$.

Outra medida particularmente conveniente, cuja objetividade decorre imediatamente de sua relação com o tensor $\mathbf{C}$, é a deformação de Green-Lagrange, definida por:

$$
\mathbf{E}=\frac{1}{2}(\mathbf{C}-\mathbf{I})
$$

Diz-se que E é normalizada, pois resulta no tensor nulo quando o corpo é sujeito apenas a movimentos de corpo rígido. Além disso, é possível verificar que essa se aproxima da clássica deformação linear de engenharia $(\varepsilon)$ à medida em que $\mathbf{A}$ se aproxima da identidade.

\subsubsection{Taxas de deformação}

O gradiente da velocidade da mudança de configuração em relação às coordenadas Eulerianas é dado por:

$$
\mathbf{L}=\nabla_{\mathbf{y}}(\dot{\boldsymbol{\zeta}})=\frac{\partial \dot{\boldsymbol{\zeta}}}{\partial \mathbf{y}}=\frac{d}{d t}\left(\frac{\partial \boldsymbol{\zeta}}{\partial \mathbf{x}}\right) \frac{\partial \mathbf{x}}{\partial \mathbf{y}}=\dot{\mathbf{A}} \mathbf{A}^{-1}
$$

onde o ponto sobrescrito $(\cdot)$ indica derivada no tempo. Pode-se ainda decompor $\mathbf{L}$ em suas 
parcelas simétrica e antissimétrica, isto é, $\mathbf{L}=\mathbf{D}+\mathbf{W}$, onde

$$
\begin{aligned}
& \mathbf{D}=\operatorname{sim}(\mathbf{L})=\frac{1}{2}\left(\mathbf{L}+\mathbf{L}^{T}\right), \quad \mathrm{e} \\
& \mathbf{W}=\operatorname{ant}(\mathbf{L})=\frac{1}{2}\left(\mathbf{L}-\mathbf{L}^{T}\right) .
\end{aligned}
$$

O tensor $\mathbf{D}$ é conhecido como taxa de deformação de engenharia, enquanto $\mathbf{W}$ é denominado tensor taxa de rotação, ou vorticidade. Aplicando (2.6) em (2.7), chega-se à relação:

$$
\mathbf{D}=\frac{1}{2}\left(\dot{\mathbf{A}} \mathbf{A}^{-1}+\mathbf{A}^{-T} \dot{\mathbf{A}}^{T}\right)=\mathbf{A}^{-T}\left[\frac{1}{2}\left(\mathbf{A}^{T} \dot{\mathbf{A}}+\dot{\mathbf{A}}^{T} \mathbf{A}\right)\right] \mathbf{A}^{-1}=\mathbf{A}^{-T} \dot{\mathbf{E}} \mathbf{A}^{-1} .
$$

Logo, a taxa de deformação de Green-Lagrange pode ser escrita como

$$
\dot{\mathbf{E}}=\mathbf{A}^{T} \mathbf{D A} .
$$

\subsubsection{Mudança de volume}

Supondo que a partir da configuração indeformada de um sólido seja extraído um cubo infinitesimal cujas dimensões são definidas por vetores $d \mathbf{x}^{1}, d \mathbf{x}^{2}$ e $d \mathbf{x}^{3}$, conforme a Figura 2. Então sabe-se que o volume do cubo é dado por

$$
d V^{0}=d \mathbf{x}^{1}\left(d \mathbf{x}^{2} \times d \mathbf{x}^{3}\right)=\operatorname{det}\left(d \mathbf{x}^{1}, d \mathbf{x}^{2}, d \mathbf{x}^{3}\right) .
$$

Suponha que o mesmo cubo tenha sofrido uma mudança de configuração de forma que venha a ser definido pelos vetores $d \mathbf{y}^{1}=\mathbf{A} d \mathbf{x}^{1}, d \mathbf{y}^{2}=\mathbf{A} d \mathbf{x}^{2}$ e $d \mathbf{y}^{3}=\mathbf{A} d \mathbf{x}^{3}$. Então o volume deformado é dado por

$$
d V=\operatorname{det}\left(d \mathbf{y}^{1}, d \mathbf{y}^{2}, d \mathbf{y}^{3}\right)=\operatorname{det}(\mathbf{A}) \operatorname{det}\left(d \mathbf{x}^{1}, d \mathbf{x}^{2}, d \mathbf{x}^{3}\right)=J d V^{0},
$$

onde

$$
J=\operatorname{det}(\mathbf{A})=\frac{d V}{d V^{0}}>0
$$

é chamado de jacobiano, ou deformação volumétrica. Partindo da hipótese de que o material não pode ser invertido ou desaparecer, isto é, que o volume $d V$ não pode vir a ser negativo ou nulo, a condição $J>0$ deve ser levada em conta no modelo do material.

Figura 2 - Configurações indeformada e deformada de um cubo infinitesimal

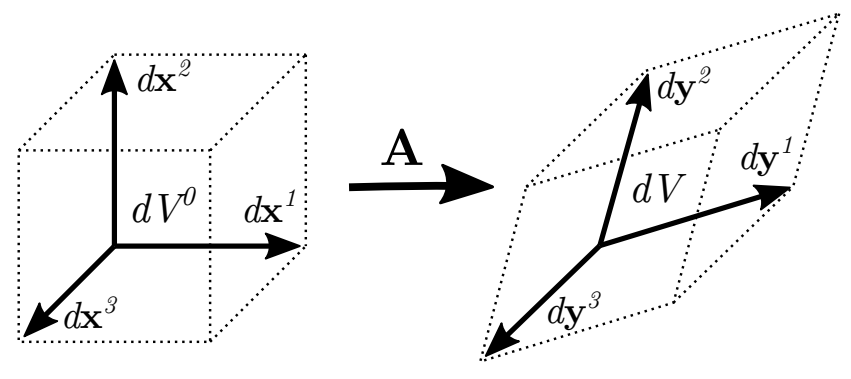

\subsubsection{Fórmula de Nanson}

Seja $d V^{0}$ um volume infinitesimal na configuração inicial definido pela projeção de uma área infinitesimal $d S^{0}$ através de um vetor $d \mathbf{x}$, e seja $\mathbf{n}^{0}$ o vetor unitário normal à 
face $d S^{0}$, conforme a Figura 3. Assim, pode-se escrever:

$$
d V^{0}=d \mathbf{x} \mathbf{n}^{0} d S^{0} .
$$

Suponha que esse corpo sofra uma mudança de configuração tal que venha a ser definido por uma nova área infinitesimal $d S$, com vetor normal unitário $\mathbf{n}$, projetada através de um vetor $d \mathbf{y}$. Então o novo volume é dado por

$$
d V=d \mathbf{y} \mathbf{n} d S=\mathbf{A} d \mathbf{x} \mathbf{n} d S .
$$

Logo, aplicando-se a fórmula da mudança de volume (2.12), resulta:

$$
\mathbf{A} d \mathbf{x} \mathbf{n} d S=d V=J d V^{0}=J d \mathbf{x} \mathbf{n}^{0} d S^{0},
$$

e, como $d \mathbf{x}$ pode ser tomado arbitrariamente, podemos escrever

$$
\begin{aligned}
& \mathbf{A} \mathbf{n} d S=J \mathbf{n}^{0} d S^{0}, \quad \text { ou } \\
& \mathbf{n} d S=J \mathbf{A}^{-1} \mathbf{n}^{0} d S^{0},
\end{aligned}
$$

que é conhecida como fórmula de Nanson, e pode ser utilizada para relacionar as áreas entre as configurações deformada e indeformada de um sólido.

Figura 3 - Configurações indeformada e deformada de volume gerado por área infinitesimal

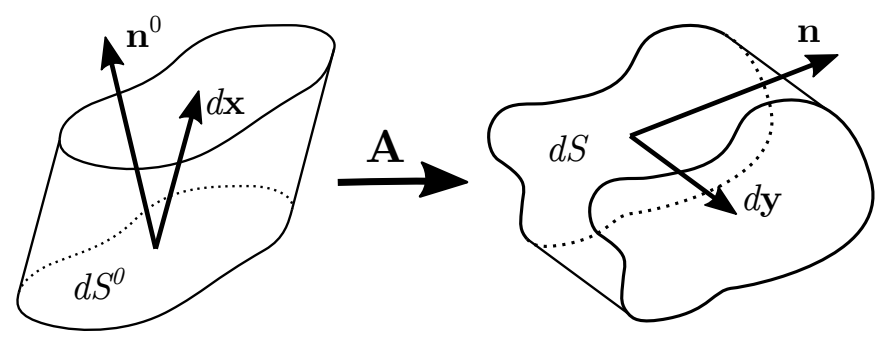

\subsubsection{Princípio da conservação da massa}

Uma propriedade fundamental para a conversão da descrição Euleriana para a Lagrangeana, bem como futuras aplicações em análises numéricas, é a conservação da massa, ou seja, a propriedade do corpo que garante que a massa $M$, escrita como

$$
M=\int_{\Omega(t)} \rho d V
$$

é constante ao longo da análise. Observe que, como o domínio $\Omega(t)$ varia com o tempo, para que a equação (2.19) permaneça constante, a massa específica $\rho$ também deve variar ao longo do tempo. Em particular,

$$
M=\int_{\Omega(t)} \rho d V=\int_{\Omega^{0}} \rho_{0} d V^{0}
$$

onde $\Omega^{0}$ é o domínio em sua configuração indeformada e $\rho_{0}$ é a massa específica inicial do sólido. Substituindo a fórmula da mudança de volume em (2.20), escreve-se:

$$
M=\int_{\Omega^{0}} J \rho d V^{0}=\int_{\Omega^{0}} \rho_{0} d V
$$


ou seja, $\rho_{0}$ e $\rho$ relacionam-se pela expressão

$$
\rho_{0}=J \rho .
$$

Logo, sendo $f$ uma função qualquer dependente do tempo, tem-se que

$$
\int_{\Omega(t)} \rho f d V=\int_{\Omega^{0}} \rho f J d V^{0}=\int_{\Omega^{0}} \rho_{0} f d V^{0}
$$

e, consequentemente,

$$
\frac{d}{d t} \int_{\Omega(t)} \rho f d V=\frac{d}{d t} \int_{\Omega^{0}} \rho_{0} f d V^{0}=\int_{\Omega^{0}} \rho_{0} \dot{f} d V^{0} .
$$

A expressão (2.24), que parte apenas do princípio da conservação de massa, será de particular utilidade na obtenção de equilíbrio dinâmico, bem como em demais passagens deste trabalho.

\subsection{Equilíbrio}

Conforme mencionado anteriormente, a formulação adotada neste trabalho utiliza uma descrição Lagrangeana total. Entretanto, deve-se observar que o estado de equilíbrio está diretamente relacionado com a configuração atual do corpo. Dessa forma, seguindo Coda (2018), inicia-se introduzindo as equações de equilíbrio em sua forma Euleriana, e, em seguida, utilizam-se as fórmulas da mudança de volume e de Nanson para obter as formas Lagrangeanas.

\subsubsection{Equilíbrio Euleriano}

Seja um corpo $\Omega^{1}$ em sua configuração atual equilibrada, sujeito a um corte imaginário conforme a Figura 4. Pela segunda lei de Newton, devem haver esforços na seção capazes de manter o equilíbrio, resultando nas chamadas tensões.

Figura 4 - Corte imaginário na configuração equilibrada de um sólido

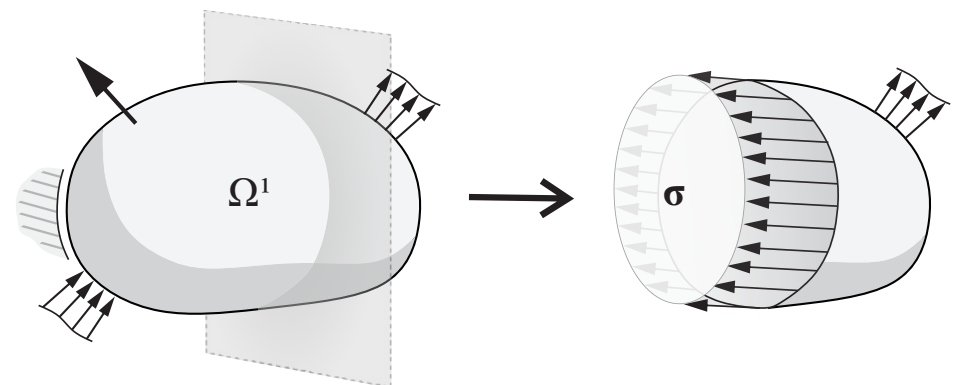

Suponha agora que esse corpo é submetido a seis cortes ortogonais aos eixos coordenados, com distâncias infinitesinais entre si, de forma que resulte um cubo infinitesimal conforme a Figura 5. Cada face do cubo está sujeita a uma tensão que pode ser decomposta em três componentes, denotadas por $\sigma_{i j}$, onde $i$ representa o eixo ortogonal a essa face e $j$ representa a direção da componente.

Nessa decomposição, as tensões ortogonais à face do cubo são chamadas tensões 
Figura 5 - Cubo infinitesimal sujeito a um estado de tensões

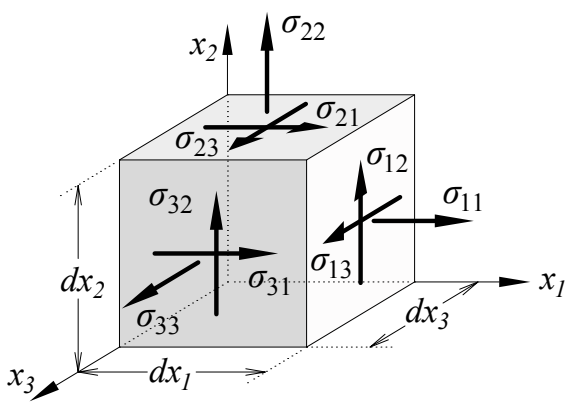

normais, enquanto aquelas tangentes ao cubo são chamadas tensões cisalhantes. Uma representação conveniente do estado de tensão dado pelo procedimento exposto é o chamado tensor de tensões de Cauchy, dado por

$$
\boldsymbol{\sigma}=\left[\begin{array}{lll}
\sigma_{11} & \sigma_{12} & \sigma_{13} \\
\sigma_{21} & \sigma_{22} & \sigma_{23} \\
\sigma_{31} & \sigma_{32} & \sigma_{33}
\end{array}\right]
$$

A tensão de Cauchy é muitas vezes chamada de tensão real, por ser aquela que efetivamente equilibra as forças externas que atuam sobre o corpo deformado. Esse aspecto deve ser frisado pois ao decorrer do texto serão utilizadas outras medidas de tensão que não apresentam significado físico explícito, embora também sirvam como ferramentas para obtenção do equilíbrio.

Realizando o equilíbrio de momentos no cubo infinitesimal, obtem-se o chamado teorema de Cauchy, que implica na simetria do tensor de tensões de Cauchy, isto é,

$$
\boldsymbol{\sigma}=\boldsymbol{\sigma}^{T}
$$

A componente $\sigma_{i j}$ denota a tensão na direção $j$ atuante em uma face ortogonal ao eixo $i$. Já na face oposta, escreve-se a tensão como $\sigma_{i j}+\sigma_{i j, i} d y_{i}$, onde $\sigma_{i j, i} d y_{i}$ é a variação infinitesimal de tensão que ocorre ao longo do cubo. Seja $\mathbf{b}$ a força de volume ao longo do elemento infinitesimal. Pela segunda lei de Newton, escreve-se:

$$
\sigma_{i j} d S_{i}-\left(\sigma_{i j}+\sigma_{i j, i} d y_{i}\right) d S_{i}+b_{j} d V=\rho \ddot{y}_{j} d V,
$$

onde $d S_{i}$ é o valor da área da face ortogonal ao eixo $i, d V=d y_{1} d y_{2} d y_{3}$ é o volume do cubo, $\rho$ é a massa específica e $\ddot{\mathbf{y}}$ é a aceleração. Desenvolvendo algebricamente a expressão (2.27), obtem-se o equilíbrio local Euleriano:

$$
\nabla_{\mathbf{y}} \cdot \boldsymbol{\sigma}+\mathbf{b}=\rho \ddot{\mathbf{y}} .
$$

onde $\nabla_{\mathbf{y}}$ denota o gradiente com relação à configuração deformada. Integrando (2.28) no domínio $\Omega^{1}$, obtém-se

$$
\int_{\Omega^{1}} \nabla_{\mathbf{y}} \cdot \boldsymbol{\sigma} d V+\int_{\Omega^{1}} \mathbf{b} d V=\int_{\Omega^{1}} \rho \ddot{\mathbf{y}} d V,
$$

de onde, utilizando o teorema de Gauss na primeira parcela, escreve-se

$$
\int_{\Gamma^{1}} \boldsymbol{\sigma} \cdot \mathbf{n} d S+\int_{\Omega^{1}} \mathbf{b} d V=\int_{\Omega^{1}} \rho \ddot{\mathbf{y}} d V,
$$


no qual $\Gamma^{1}$ representa o contorno de $\Omega^{1}$, e $\mathbf{n}$ o versor normal a $\Gamma^{1}$ no ponto analisado. $\mathrm{O}$ termo $\boldsymbol{\sigma} \cdot \mathbf{n}$ também pode ser visto como uma força de superfície, sendo denotado por $\mathbf{p}$. Logo, o equilíbrio global Euleriano pode ser escrito como:

$$
\int_{\Gamma^{1}} \mathbf{p} d S+\int_{\Omega^{1}} \mathbf{b} d V=\int_{\Omega^{1}} \rho \ddot{\mathbf{y}} d V .
$$

\subsubsection{Equilíbrio Lagrangeano}

Aplicando a fórmula de Nanson (2.18) na primeira parcela de (2.30) e a fórmula da mudança de volume (2.12) nas demais parcelas, obtém-se

$$
\int_{\Gamma^{0}}\left(J \boldsymbol{\sigma} \mathbf{A}^{-1}\right) \cdot \mathbf{n}^{0} d S^{0}+\int_{\Omega^{0}} J \mathbf{b} d V^{0}=\int_{\Omega^{0}} J \rho \ddot{\mathbf{y}} d V^{0},
$$

onde $\Gamma^{0}$ representa o contorno de $\Omega^{0}$. Pelo princípio da conservação da massa, podemos substituir $J \rho$ na terceira parcela por $\rho_{0}$. Supondo ainda que as forças de volume sejam conservativas, podemos utilizar um argumento análogo ao princípio da conservação da massa e substituir $J \mathbf{b}$ por uma força de volume Lagrangeana, denotada por $\mathbf{b}^{0}$. Além disso, define-se o tensor de tensões de Piola-Kirchhoff de primeira espécie como

$$
\mathbf{P}=J \mathbf{A}^{-T} \boldsymbol{\sigma} .
$$

Dessa forma, a equação (2.32) pode ser escrita como

$$
\int_{\Gamma^{0}} \mathbf{P}^{T} \cdot \mathbf{n}^{0} d S^{0}+\int_{\Omega^{0}} J \mathbf{b}^{0} d V^{0}=\int_{\Omega^{0}} \rho_{0} \ddot{\mathbf{y}} d V^{0}
$$

que representa o equilíbrio global Lagrangeano. Aplicando o teorema de Gauss e a arbitrariedade do volume, pode-se obter o equilíbrio local Lagrangeano pela expressão

$$
\nabla_{\mathbf{x}} \cdot \mathbf{P}^{T}+\mathbf{b}^{0}=\rho_{0} \ddot{\mathbf{y}}
$$

mostrando que o equilíbrio pode ser obtido em termos do tensor de Piola-Kirchhoff de primeira espécie.

\subsubsection{Equilíbrio Lagrangeano por abordagem energética}

Neste trabalho, utiliza-se como base para obtenção do equilíbrio a abordagem energética, na qual escreve-se o funcional da energia mecânica total do sistema como

$$
\Pi=\Pi_{d e f}+\Pi_{e x t}+\Pi_{c i n}
$$

onde $\Pi_{\text {def }}$ é a energia de deformação, $\Pi_{e x t}$ é a energia potencial das forças externas e $\Pi_{\text {cin }}$ é a energia cinética. O equilíbrio é então obtido pelo princípio da energia mecânica estacionária total, que estabelece que a primeira variação do funcional deve ser nula, isto é,

$$
\delta \Pi=\delta \Pi_{d e f}+\delta \Pi_{e x t}+\delta \Pi_{c i n}=0 .
$$

Com base em (2.35), escreve-se:

$$
\delta \Pi=\left(\rho_{0} \ddot{\mathbf{y}}-\nabla_{\mathbf{x}} \cdot \mathbf{P}^{T}-\mathbf{b}^{0}\right) \delta \mathbf{y},
$$

onde $\delta \mathbf{y}$ representa uma variação arbitrária no campo de posições y. Integrando (2.38) no volume inicial e realizando algumas manipulações algébricas, é possível mostrar que essa 
pode ser escrita na forma (CODA, 2018):

$$
\delta \Pi=\int_{\Omega^{0}} \rho_{0} \ddot{\mathbf{y}} \delta \mathbf{y} d V^{0}+\int_{\Omega^{0}} \mathbf{P}^{T}: \delta \mathbf{A} d V^{0}-\int_{\Gamma^{0}} \mathbf{p}^{0} \delta \mathbf{y} d S^{0}-\int_{\Omega^{0}} \mathbf{b}^{0} \delta \mathbf{y} d V^{0}=0,
$$

onde $\mathbf{p}^{0}$ representa a força de superfície Lagrangeana, também assumida conservativa.

Diz-se, portanto, que $\mathbf{P}^{T}$ é conjugado energético de $\mathbf{A}$. A equação (2.39), no entanto, ainda não é a forma ideal da equação de equilíbrio, uma vez que A não é uma medida de deformação objetiva, e $\mathbf{P}$ apresenta diversas desvantagens enquanto medida de tensão, incluindo o fato de ser não-simétrico. Dessa forma, define-se o tensor de tensões de Piola-Kirchhoff de segunda espécie:

$$
\mathbf{S}=\mathbf{A}^{-1} \mathbf{P}^{T}
$$

Combinando (2.40) e (2.33), segue que o tensor $\mathbf{S}$ relaciona-se com a tensão de Cauchy pela expressão

$$
\boldsymbol{\sigma}=\frac{1}{J} \mathbf{A S A}^{T}
$$

sendo, portanto, simétrico. Aplicando (2.40) e a relação tensorial (A.32), pode-se escrever

$$
\mathbf{P}^{T}: \delta \mathbf{A}=(\mathbf{A} \mathbf{S}): \delta \mathbf{A}=\mathbf{S}:\left(\mathbf{A}^{T} \delta \mathbf{A}\right) .
$$

Da simetria de $\mathbf{S}$, e pela relação tensorial (A.40), conclui-se que:

$$
\mathbf{S}:\left(\mathbf{A}^{T} \delta \mathbf{A}\right)=\mathbf{S}: \operatorname{sim}\left(\mathbf{A}^{T} \delta \mathbf{A}\right)=\mathbf{S}: \frac{1}{2}\left(\mathbf{A}^{T} \delta \mathbf{A}+\mathbf{A} \delta \mathbf{A}^{T}\right)=\mathbf{S}: \delta \mathbf{E},
$$

logo, S é conjugado energético da deformação de Green-Lagrange, E, e a equação (2.39) pode ser escrita como

$$
\delta \Pi=\int_{\Omega^{0}} \rho_{0} \ddot{\mathbf{y}} \delta \mathbf{y} d V^{0}+\int_{\Omega^{0}} \mathbf{S}: \delta \mathbf{E} d V^{0}-\int_{\Gamma^{0}} \mathbf{p}^{0} \delta \mathbf{y} d S^{0}-\int_{\Omega^{0}} \mathbf{b}^{0} \delta \mathbf{y} d V^{0}=0 .
$$

Resta, então, definir $\Pi$ de forma que a equação (2.44) seja atendida.

\subsubsection{Energia cinética}

Escreve-se a energia cinética como

$$
\Pi_{c i n}=\frac{1}{2} \int_{\Omega(t)} \rho(t) \dot{\mathbf{y}} \dot{\mathbf{y}} d V=\frac{1}{2} \int_{\Omega^{0}} \rho_{0} \dot{\mathbf{y}} \dot{\mathbf{y}} d V^{0}
$$

onde utilizou-se o teorema da conservação da massa na segunda passagem. Disso resulta

$$
\delta \Pi_{\text {cin }}=\frac{d \Pi_{\text {cin }}}{d t} d t=\frac{1}{2} \int_{\Omega^{0}} \rho_{0}(2 \ddot{\mathbf{y}} \dot{\mathbf{y}}) d t d V^{0}=\int_{\Omega^{0}} \rho_{0} \ddot{\mathbf{y}}(\dot{\mathbf{y}} d t) d V^{0}=\int_{\Omega^{0}} \rho_{0} \ddot{\mathbf{y}} \delta \mathbf{y} d V^{0},
$$

que equivale à primeira parcela da equação (2.44).

\subsubsection{Energia potencial das forças externas}

Escreve-se a energia potencial das forças externas como

$$
\Pi_{\text {ext }}=-\int_{\Gamma^{0}} \mathbf{p}^{0} \cdot \mathbf{y} d S^{0}-\int_{\Omega^{0}} \mathbf{b}^{0} \cdot \mathbf{y} d V^{0},
$$

onde a primeira parcela refere-se às forças de superfície, e a segunda às forças de volume. 
Segue de imediato que

$$
\delta \Pi_{e x t}=-\int_{\Gamma^{0}} \mathbf{p}^{0} \cdot \delta \mathbf{y} d S^{0}-\int_{\Omega^{0}} \mathbf{b}^{0} \cdot \delta \mathbf{y} d V^{0} .
$$

Portanto, a energia potencial das forças externas resulta na terceira e quarta parcela de (2.44), levando à conclusão de que, para haver equivalência entre a abordagem energética e o equilíbrio Lagrangeano, a segunda parcela deve ser equivalente a $\delta \Pi_{d e f}$.

\subsubsection{Energia de deformação}

Escreve-se a energia de deformação como

$$
\Pi_{d e f}=\int_{\Omega^{0}} \Psi d V^{0}
$$

onde $\Psi$, denominada energia específica de deformação ou energia livre de Helmholtz, estabelece a relação constitutiva do material, isto é, a lei que relaciona tensões e deformações. Para que a equação (2.49) resulte na segunda parcela de (2.39) ou (2.44), deve-se ter

$$
\delta \Pi_{d e f}=\int_{\Omega^{0}} \delta \Psi d V^{0}=\int_{\Omega^{0}} \mathbf{P}^{T}: \delta \mathbf{A} d V^{0}=\int_{\Omega^{0}} \mathbf{S}: \delta \mathbf{E} d V^{0}=\frac{1}{2} \int_{\Omega^{0}} \mathbf{S}: \delta \mathbf{C} d V^{0},
$$

ou, pela arbitrariedade do volume,

$$
\delta \Psi=\mathbf{P}^{T}: \delta \mathbf{A}=\mathbf{S}: \delta \mathbf{E}=\frac{1}{2} \mathbf{S}: \delta \mathbf{C} .
$$

Dessa forma, as medidas de tensão $\mathbf{P}$ e $\mathbf{S}$ podem ser calculadas, respectivamente, pelas expressões

$$
\begin{gathered}
\mathbf{P}=\frac{\partial \Psi}{\partial \mathbf{A}^{T}}, \quad \mathrm{e} \\
\mathbf{S}=\frac{\partial \Psi}{\partial \mathbf{E}}=2 \frac{\partial \Psi}{\partial \mathbf{C}} .
\end{gathered}
$$

Logo, escrevendo $\Psi$ em função das deformações, e sabendo que essas dependem apenas das posições, pode-se definir a energia mecânica total $\Pi$ e, consequentemente, o equilíbrio, totalmente em função de $\mathbf{y}, \dot{\mathbf{y}}$ e $\ddot{\mathbf{y}}$.

\subsection{Invariantes dos tensores de tensão e deformação}

Seja T um tensor genérico representando o estado de deformações ou tensões em um determinado ponto. Sabe-se que T não é a única representação desse estado, pois depende da escolha dos eixos coordenados (isso pode ser visto, por exemplo, na forma como a tensão de Cauchy é definida na subseção 2.2.1). Logo, a fim de caracterizar o estado de tensões de maneira independente à essa escolha de eixos, utilizam-se os chamados invariantes:

$$
\left\{\begin{array}{l}
I_{1}=\operatorname{tr}(\mathbf{T}) \\
I_{2}=\frac{1}{2}\left[\operatorname{tr}(\mathbf{T})^{2}-\operatorname{tr}\left(\mathbf{T}^{2}\right)\right], \\
I_{3}=\operatorname{det}(\mathbf{T})
\end{array}\right.
$$


onde $\operatorname{tr}(\bullet)$ simboliza o traço de um tensor, isto é, a soma dos termos da diagonal principal:

$$
\operatorname{tr}(\mathbf{T})=\mathbf{I}: \mathbf{T}=T_{11}+T_{22}+T_{33}
$$

É possível mostrar que $I_{1}, I_{2}$ e $I_{3}$ independem de rotações, e, portanto, possuem o mesmo valor para diferentes representações do mesmo estado.

\subsection{Tensões hidrostráticas e desviadoras}

Seja $\mathbf{T}$ um tensor de tensões genérico e $I_{1}$ o seu primeiro invariante, isto é, $I_{1}=\operatorname{tr}(\mathbf{T})$. Definem-se as tensões hidrostáticas e desviadoras, respectivamente, como (BONET; WOOD, 1997):

$$
\begin{gathered}
\mathbf{T}^{H}=\frac{I_{1}}{3} \mathbf{I}, \quad \mathrm{e} \\
\mathbf{T}^{D}=\mathbf{T}-\mathbf{T}^{H}=\mathbf{T}-\frac{I_{1}}{3} \mathbf{I} .
\end{gathered}
$$

A tensão hidrostática está diretamente associada com a mudança de volume, enquanto a tensão desviadora está associada à distorção pura. Dessa forma, a decomposição $\mathbf{T}=\mathbf{T}^{H}+\mathbf{T}^{D}$ é conveniente em diversos contextos onde é necessária uma distinção entre os efeitos volumétricos e isocóricos.

\subsection{Modelos constitutivos hiperelásticos}

Conforme estabelecido na subseção 2.2.3.3, os modelos constitutivos são definidos pela energia livre de Helmholtz, $\Psi$. Diz-se que o modelo é hiperelástico quando, além de ser elástico, sua expressão para $\Psi$ é definida em função apenas do estado de deformação atual, e independe de fatores como histórico e taxa de deformação. $\mathrm{O}$ valor de $\Psi$ nesse caso pode ser escrito tanto em função de $\mathbf{E}$ quanto de $\mathbf{C}$, uma vez que a equação (2.53) pode ser utilizada para relacionar as duas situações. Entretanto, em leis ditas isotrópicas, $\Psi$ deve ser definida de forma que independa da escolha dos eixos coordenados. Para garantir que isso ocorra, é conveniente escrever as leis hiperelásticas em função dos invariantes de E ou C, isto é,

$$
\Psi(\mathbf{E})=\Psi(\mathbf{C})=\Psi\left(I_{1}, I_{2}, I_{3}\right) .
$$

Além disso, espera-se que $\Psi$ seja normalizada, ou seja,

$$
\mathbf{E}=\mathbf{0} \Rightarrow \Psi=0
$$

e, em caso de grandes deformações, respeite as condições de crescimento

$$
\lim _{J \rightarrow 0^{+}} \Psi=\lim _{J \rightarrow+\infty} \Psi=+\infty,
$$

onde $J=\sqrt{I_{3}}=\sqrt{\operatorname{det}(\mathbf{C})}$ é o jacobiano, ou deformação volumétrica, definido em (2.13). 


\subsubsection{Lei de Saint Venant-Kirchhoff}

A lei constitutiva de Saint-Vennant Kirchhoff (SVK) é considerada o caso mais simples de lei hiperelástica, sendo aplicável apenas em casos de pequenas deformações. Essa lei é definida pela energia livre de Helmholtz:

$$
\Psi=\frac{1}{2} \mathbf{E}: \mathfrak{C}^{e}: \mathbf{E},
$$

que resulta em uma relação linear entre tensões e deformações:

$$
\mathbf{S}=\mathfrak{C}^{e}: \mathbf{E}
$$

onde $\mathfrak{C}^{e}$ é um tensor constitutivo de quarta ordem, que depende apenas dos parâmetros do material. No caso de sólidos tridimensionais isótropos, $\mathfrak{C}^{e}$ é dado por

$$
\mathfrak{C}^{e}=2 G \mathbf{I I}+\lambda \mathbf{I} \otimes \mathbf{I},
$$

onde $G$ e $\lambda$ são o módulo de elasticidade transversal e a constante de Lamé, que podem ser calculados a partir do módulo de Young $E$ e coeficiente de poisson $\nu$ pelas expressões:

$$
\lambda=\frac{\nu E}{(1+\nu) \cdot(1-2 \nu)} \quad \text { e } \quad G=\frac{E}{2(1+\nu)} .
$$

Aplicando (2.63) em (2.62), obtém-se a seguinte relação entre tensão de PiolaKirchhoff de segunda espécie e a deformação de Green-Lagrange:

$$
\mathbf{S}=2 G \mathbf{E}+\lambda \operatorname{tr}(\mathbf{E}) \mathbf{I} .
$$

\subsubsection{Representação Matricial}

A representação (2.65) traz vantagens no equacionamento, porém, para fins de facilitar a visualização, pode ser empregada a notação de Voigt, onde as tensões e deformações são representadas por vetores, e o operador constitutivo linear por uma matriz. Nesse contexto, a lei de Saint Venant-Kirchhoff pode ser expressa como

$$
\left\{\begin{array}{l}
S_{11} \\
S_{22} \\
S_{33} \\
S_{12} \\
S_{13} \\
S_{23}
\end{array}\right\}=\left[\begin{array}{cccccc}
2 G+\lambda & \lambda & \lambda & 0 & 0 & 0 \\
\lambda & 2 G+\lambda & \lambda & 0 & 0 & 0 \\
\lambda & \lambda & 2 G+\lambda & 0 & 0 & 0 \\
0 & 0 & 0 & 2 G & 0 & 0 \\
0 & 0 & 0 & 0 & 2 G & 0 \\
0 & 0 & 0 & 0 & 0 & 2 G
\end{array}\right]\left\{\begin{array}{l}
E_{11} \\
E_{22} \\
E_{33} \\
E_{12} \\
E_{13} \\
E_{23}
\end{array}\right\} .
$$

Para sólidos bidimensionais sob estado plano de deformações, a relação constitutiva é representada por

$$
\left\{\begin{array}{l}
S_{11} \\
S_{22} \\
S_{12}
\end{array}\right\}=\left[\begin{array}{ccc}
2 G+\lambda & \lambda & 0 \\
\lambda & 2 G+\lambda & 0 \\
0 & 0 & 2 G
\end{array}\right]\left\{\begin{array}{l}
E_{11} \\
E_{22} \\
E_{12}
\end{array}\right\}
$$


e, para o estado plano de tensões,

$$
\left\{\begin{array}{l}
S_{11} \\
S_{22} \\
S_{12}
\end{array}\right\}=\left[\begin{array}{ccc}
2 G+\bar{\lambda} & \bar{\lambda} & 0 \\
\bar{\lambda} & 2 G+\bar{\lambda} & 0 \\
0 & 0 & 2 G
\end{array}\right]\left\{\begin{array}{l}
E_{11} \\
E_{22} \\
E_{12}
\end{array}\right\},
$$

onde

$$
\bar{\lambda}=\frac{\nu E}{1-\nu^{2}}
$$

\subsubsection{Prova da isotropia}

Na expressão (2.61) a energia livre de Helmholtz foi escrita em função da deformação E, e portanto, a princípio, não é garantida a isotropia da lei. Para prova-la, escreve-se $\Psi$ em termos dos invariantes $I_{1}$ e $I_{2}$ de $\mathbf{E}$, a partir da expressão

$$
\Psi=G I_{2}+\frac{\lambda-G}{2} I_{1}^{2}=G \operatorname{tr}\left(\mathbf{E}^{2}\right)+\frac{\lambda}{2} \operatorname{tr}(\mathbf{E})^{2} .
$$

Observando-se que (ver (A.20) para detalhes sobre as operações tensoriais):

$$
\frac{\partial \operatorname{tr}(\mathbf{E})^{2}}{\partial \mathbf{E}}=2 \operatorname{tr}(\mathbf{E}) \frac{\partial \operatorname{tr}(\mathbf{E})}{\partial \mathbf{E}}=2 \operatorname{tr}(\mathbf{E}) \mathbf{I},
$$

e, considerando-se a simetria de $\mathbf{E}(\operatorname{ver}($ A.16) e (A.20)):

$$
\frac{\partial \operatorname{tr}\left(\mathbf{E}^{2}\right)}{\partial E_{i j}}=\frac{\partial \operatorname{tr}\left(\mathbf{E}^{2}\right)}{\partial E_{k l}^{2}} \frac{\partial\left(E_{k l}^{2}\right)}{\partial E_{i j}}=\delta_{k l}\left(\delta_{k i} E_{j l}+E_{k i} \delta_{l j}\right)=E_{j i}+E_{j i}=2 E_{i j} .
$$

temos que a tensão pode ser calculada como

$$
\mathbf{S}=\frac{\partial \Psi}{\partial \mathbf{E}}=G \frac{\partial \operatorname{tr}\left(\mathbf{E}^{2}\right)}{\partial \mathbf{E}}+\frac{\lambda}{2} \frac{\partial \operatorname{tr}(\mathbf{E})^{2}}{\partial \mathbf{E}}=2 G \mathbf{E}+\lambda \operatorname{tr}(\mathbf{E}) \mathbf{I}
$$

que equivale à expressão (2.65), mostrando que (2.61) e (2.70) representam a mesma lei, definida em termos dos invariantes e, portanto, isotrópica.

\subsubsection{Condições de crescimento}

Embora a lei de Saint Venant-Kirchhoff seja isotrópica e claramente normalizada, ela não atende às condições de crescimento. Para demonstrar tal fato, considere uma barra de treliça alinhada ao eixo 1 , com comprimento inicial $\ell_{0}$, deformada de maneira a assumir o comprimento $\ell$. Nesse caso pode-se verificar que em cada ponto da barra temos

$$
\begin{gathered}
J=A_{11}=\frac{\ell}{\ell_{0}}, \quad \mathrm{e} \\
E_{11}=\frac{1}{2}\left(\frac{\ell^{2}}{\ell_{0}^{2}}-1\right)=\frac{1}{2}\left(J^{2}-1\right),
\end{gathered}
$$

e a lei de Saint Venant-Kirchhoff se resume a

$$
\begin{aligned}
& \Psi=\frac{E}{2} E_{11}^{2}=\frac{E}{8}\left(J^{2}-1\right)^{2}, \quad \mathrm{e} \\
& S_{11}=E E_{11}=\frac{E}{2}\left(J^{2}-1\right) .
\end{aligned}
$$

Os gráficos de $\Psi$ e $S_{11}$ em função do jacobiano $J$ são apresentados na Figura 6 
para $E=1$. Nesses gráficos, é evidente que a condição de crescimento não é atendida, uma vez que $\Psi$ tende para 0.125 quando $J$ tende a zero. Uma consequência disso é que o modelo SVK permite a "inversão" ou "desaparecimento" do material, como pode ser visto no gráfico da tensão: quando $S_{11}$ tende ao valor finito -0.5 , o jacobiano tende a 0 .

Figura 6 - Gráficos de energia e tensão para uma barra de treliça com modelo SVK
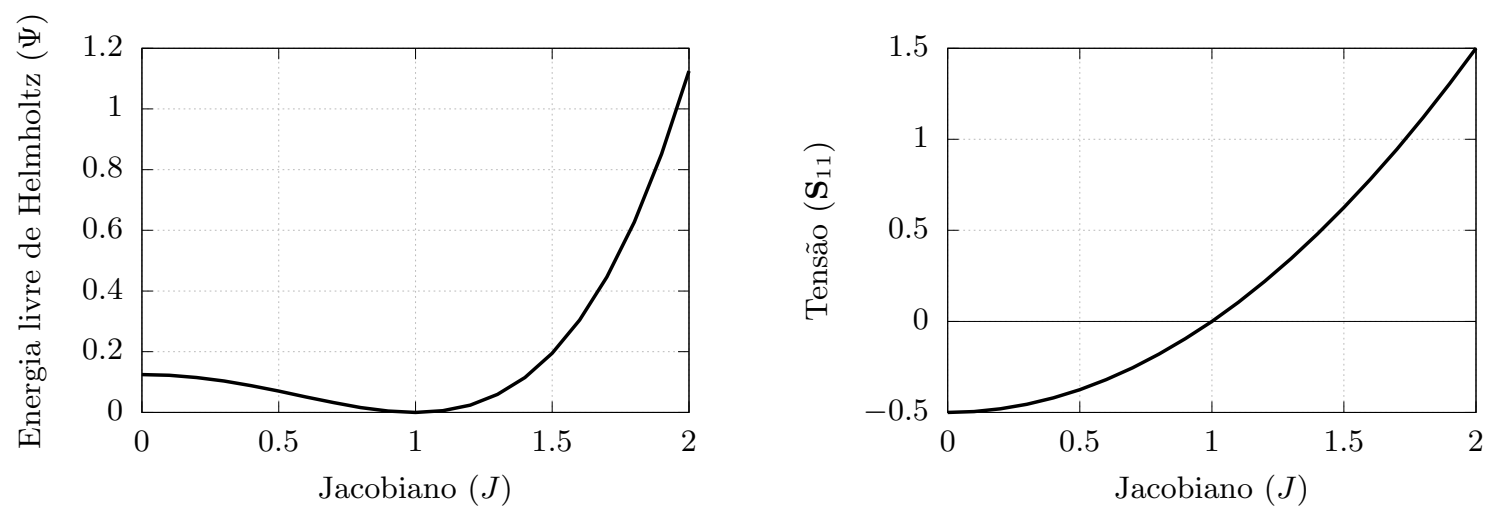

Assim, conclui-se que o modelo de Saint Venant-Kirchhoff não é adequado em casos de grandes deformações, especialmente quando há níveis elevados de compressão. Para essas situações, torna-se necessária a definição de uma lei hiperelástica que atenda às condições de crescimento. Um exemplo de modelo que relaciona-se com o de SVK no caso de pequenas deformações porém respeita as condições de crescimento é a lei Neo-Hookeana, definida na próxima seção.

\subsubsection{Modelo Neo-Hookeano}

O modelo Neo-Hookeano adotado é definido pela expressão:

$$
\Psi=\frac{\Lambda}{2} \ln (J)^{2}+\frac{\mu}{2}[\operatorname{tr}(\mathbf{C})-3-2 \ln (J)]
$$

na qual $\Lambda$ e $\mu$ são parâmetros que podem ser associados com a constante de Lamé e o módulo de elasticidade transversal, respectivamente. Observa-se $\Psi$ é isotrópica, pois é definida em função dos invariantes $I_{1}=\operatorname{tr}(\mathbf{C})$ e $J=\sqrt{\operatorname{det}(\mathbf{C})}=\sqrt{I_{3}}$. Além disso, analisando a expressão (2.78), pode-se perceber que o parâmetro $\Lambda$ serve como um penalizador para $\ln (J)$, sendo, portanto, responsável por controlar a proximidade de $J$ a 1 , ou seja, a incompressibilidade do material.

\subsubsection{Cálculo da tensão}

Para as parcelas de $\Psi$ escritas em função de $\mathbf{C}$, a expressão (2.53) pode ser utilizada para o cálculo da tensão. Para as parcelas de $\Psi$ escritas em função de $J$, utiliza-se o seguinte artifício:

$$
\mathbf{S}=2 \frac{\partial \Psi(\mathbf{C})}{\partial \mathbf{C}}=2 \frac{\partial \Psi(J)}{\partial J} \frac{\partial J}{\partial \mathbf{C}}=\frac{\partial \Psi(J)}{\partial J} J \mathbf{C}^{-1}
$$

onde na última passagem foi utilizada a equação (A.24) e o fato que $J=\sqrt{\operatorname{det}(\mathbf{C})}$. Tem-se, 
portanto:

$$
\begin{aligned}
\mathbf{S} & =\frac{\partial}{\partial \mathbf{E}}\left[\frac{\mu}{2} \operatorname{tr}(\mathbf{C})-\frac{3}{2} \mu-\mu \ln (J)+\frac{\Lambda}{2} \ln (J)^{2}\right] \\
& =\mu \frac{\partial \operatorname{tr}(\mathbf{C})}{\partial \mathbf{C}}-\mu \frac{\partial \ln (J)}{\partial J} J \mathbf{C}^{-1}+\frac{\Lambda}{2} \frac{\partial\left[\ln (J)^{2}\right]}{\partial J} J \mathbf{C}^{-1} \\
& =\mu\left(\mathbf{I}-\mathbf{C}^{-1}\right)+\Lambda \ln (J) \mathbf{C}^{-1} .
\end{aligned}
$$

\subsubsection{Cálculo do operador tangente consistente hiperelástico}

O operador tangente consistente hiperelástico, $\mathfrak{C}^{e}$, definido como

$$
\mathfrak{C}^{e}=\frac{\partial \mathbf{S}}{\partial \mathbf{E}}=2 \frac{\partial \mathbf{S}}{\partial \mathbf{C}}
$$

assume particular importância na solução numérica do problema. No caso da lei de Saint Venant-Kirchhoff esse é constante e definido previamente pelos parâmetros do material. Já no modelo Neo-Hookeano, o valor de $\mathfrak{C}^{e}$ pode mudar conforme o nível de deformação. Dessa forma, partindo de (2.80), tem-se que

$$
\begin{aligned}
\mathfrak{C}^{e} & =\frac{\partial}{\partial \mathbf{E}}\left[\mu\left(\mathbf{I}-\mathbf{C}^{-1}\right)+\Lambda \ln (J) \mathbf{C}^{-1}\right] \\
& =-2 \mu \frac{\partial \mathbf{C}^{-1}}{\partial \mathbf{C}}+\Lambda \frac{d \ln (J)}{d J} J \mathbf{C}^{-1} \otimes \mathbf{C}^{-1}+2 \Lambda \ln (J) \frac{\partial \mathbf{C}^{-1}}{\partial \mathbf{C}} \\
& =-2 \mu \frac{\partial \mathbf{C}^{-1}}{\partial \mathbf{C}}+\Lambda \mathbf{C}^{-1} \otimes \mathbf{C}^{-1}+2 \Lambda \ln (J) \frac{\partial \mathbf{C}^{-1}}{\partial \mathbf{C}}
\end{aligned}
$$

onde o tensor $\partial \mathbf{C}^{-1} / \partial \mathbf{C}$ pode ser obtido pela equação (A.18). Nota-se que, quando as deformações tendem a zero,

$$
\frac{\partial \mathbf{C}^{-1}}{\partial \mathbf{C}} \rightarrow \mathbf{I I}, \quad \mathbf{C}^{-1} \otimes \mathbf{C}^{-1} \rightarrow \mathbf{I} \otimes \mathbf{I}, \quad \text { e } \quad \ln (J) \rightarrow 0,
$$

mostrando que, quando $\Lambda=\lambda, \mu=G$, e as deformações forem suficientemente pequenas, as equações (2.82) e (2.63) podem ser associadas.

\subsubsection{Estado plano de deformações e de tensões}

Nota-se que a lei neo-Hookeana (2.78), tal como as leis apresentadas em seções posteriores, é definida para o caso 3D, e portanto, requer certas aproximações para que seja aplicada em problemas bidimensionais. Neste trabalho, utilizam-se as clássicas hipóteses de estado plano de deformações (EPD) e estado plano de tensões (EPT).

No EPD, assume-se que $E_{13}=E_{23}=E_{33}=0$, o que equivale a manter as equações (2.80) e (2.82), porém utilizando tensores $2 \times 2$ ao invés de $3 \times 3$.

Já no EPT, assume-se que $S_{13}=S_{23}=S_{33}=0$. Para materiais isotrópicos, as condições $S_{13}=S_{23}=0$ são automaticamente atendidas se $E_{13}=E_{23}=0$. Logo, resta encontrar o valor de $E_{33}$ tal que $S_{33}=0$. No caso da lei de Saint Venant-Kirchhoff, essa equação é linear, o que resulta em um valor explícito para $E_{33}$ que já é considerado na lei dada em (2.68). Já no caso neo-Hookeano, tal equação é não-linear, e, portanto, requer 
métodos de resolução mais sofisticados. Neste trabalho, utiliza-se o método numérico de Newton-Raphson, de acordo com os passos:

1. Inicialmente, assume-se um valor tentativa para $E_{33}$. Tal valor pode ser 0 ou algum valor de $E_{33}$ calculado anteriormente que pode estar próximo da solução;

2. Para essa tentativa, calcula-se $\mathbf{S}$ e $\mathfrak{C}_{3333}^{e}$;

3. Corrige-se a deformação tentativa somando o valor $\Delta E_{33}=-S_{33} / \mathfrak{C}_{3333}^{e}$;

4. Se o erro $\left|\Delta E_{33}\right|$ ou $\left|S_{33}\right|$ for menor que uma tolerância pré-estabelecida, a tentativa é dada como solução. Caso contrário, retorna-se ao Item 2.

Apesar do método apresentado ter um custo computacional maior, esse é vantajoso devido à sua generalidade, uma vez que pode ser aplicado em qualquer lei constitutiva (ZIENKIEWICZ; TAYLOR, 2005). Já a solução exata, se não impraticável, requer uma formulação diferente para cada variação do modelo. 



\section{Capítulo 3}

\section{O método dos elementos finitos posicional}

A ideia básica do MEF é a divisão do domínio em subdomínios menores, denominados elementos finitos. Tais elementos são compostos por nós, aos quais se associam as chamadas funções de forma e os parâmetros das variáveis do problema. Os campos das variáveis são então representados por uma combinação linear das funções de forma cujos coeficientes são os respectivos parâmetros nodais. Dessa maneira, um problema contínuo é aproximado pela combinação linear de funções discretas, cuja precisão da resposta depende do refinamento da malha de elementos adotada.

Adotam-se neste trabalho elementos finitos triangulares (ver Figura 7) para discretizar os sólidos bidimensionais, devido à sua melhor adequação com geometrias curvas quando comparado a outros tipos de elementos. Os elementos de 3 nós (T3), 6 nós (T6) e 10 nós (T10) possuem como funções de forma polinômios de Lagrange de ordem linear, quadrática e cúbica, respectivamente, sendo o T10 utilizado na maior parte dos exemplos. Tal escolha se justifica pelos bons resultados que tal elemento tem apresentado em trabalhos

Figura 7 - Elementos finitos triangulares em coordenadas adimensionais
(a) $\mathrm{T} 3$
(b) T6
(c) $\mathrm{T} 10$
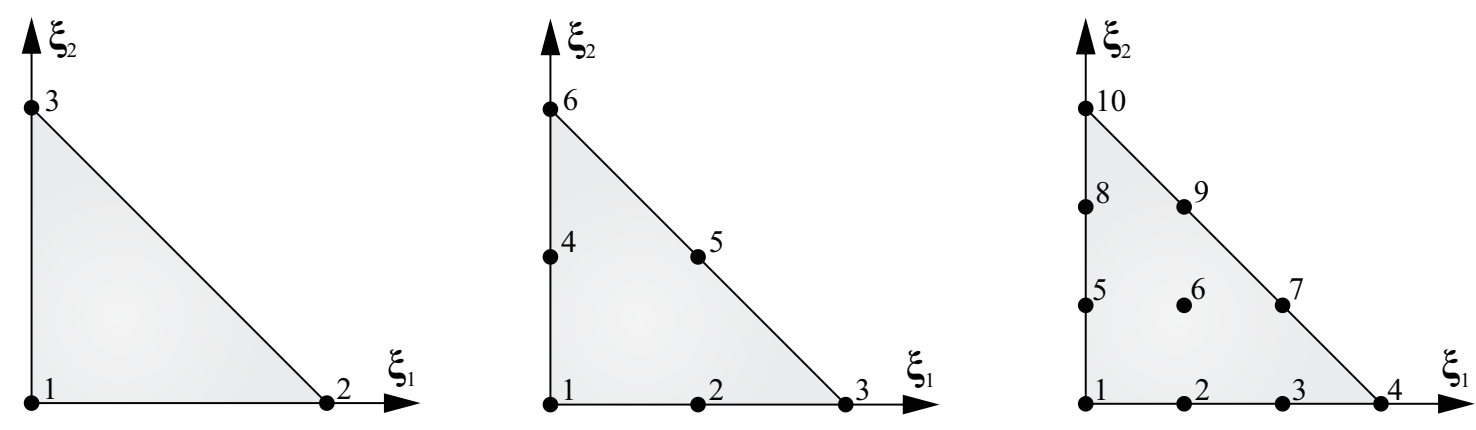

Figura 8 - Elementos finitos de linha curva em coordenadas adimensionais

(a) L2

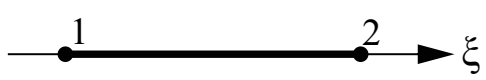

(b) L3

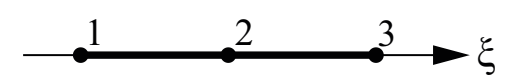

(c) L4

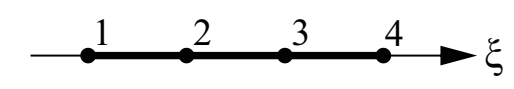


que utilizam a abordagem adotada, sendo superior a elementos de aproximação quadrática pela sua capacidade de apresentar pontos de inflexão e pela representação geométrica mais adequada de sólidos cujo contorno seja curvilíneo ou circular. Para discretizar o contorno dos sólidos, aplicam-se os elementos de linha curva de grau equivalente, conforme a Figura 8.

Neste trabalho, assim como em grande parte dos trabalhos voltados para análise de problemas dependentes do tempo, a técnica de elementos finitos é utilizada apenas para discretização espacial, sendo aplicado um processo de marcha no tempo para discretização temporal. Isso ocorre mesmo em casos onde a análise é estática ou quase-estática (efeitos inerciais desprezíveis), para que se possa levar em consideração dependências temporais do carregamento, e/ou para que o processo de solução não-linear seja facilitado, no que tange à evolução do modelo constitutivo. Nesse caso, bem como em análises dinâmicas, a precisão da resposta depende do refinamento dos passos de tempo.

Em abordagens tradicionais do MEF aplicado à mecânica dos sólidos, as variáveis nodais a serem calculadas são os deslocamentos. Já na abordagem posicional, elaborada por Bonet et al. (2000) e Coda (2003), utilizam-se como variáveis as posições dos nós com relação a um sistema de referência. A formulação descrita neste capítulo é baseada em Coda (2018). Para mais detalhes, o mesmo pode ser consultado.

Denota-se por $\mathbf{x}^{\alpha}$ o vetor contendo as posições iniciais do nó $\alpha$, e por $\mathbf{y}^{\alpha}$ o vetor que contém as posições atuais do mesmo nó, cujo conhecimento é de interesse na análise. As posições iniciais e atuais ao longo do elemento podem ser mapeadas pelas funções $\boldsymbol{\zeta}^{0}$ e $\zeta^{1}$ a partir de um domínio paramétrico adimensional auxiliar de um elemento finito (ver Figura 9), pelas expressões:

$$
\begin{aligned}
& \boldsymbol{\zeta}^{0}(\boldsymbol{\xi})=\varphi^{\alpha}(\boldsymbol{\xi}) \mathbf{x}^{\alpha}, \quad \mathrm{e} \\
& \boldsymbol{\zeta}^{1}(\boldsymbol{\xi})=\varphi^{\alpha}(\boldsymbol{\xi}) \mathbf{y}^{\alpha},
\end{aligned}
$$

Figura 9 - Mapeamento das configurações iniciais e finais

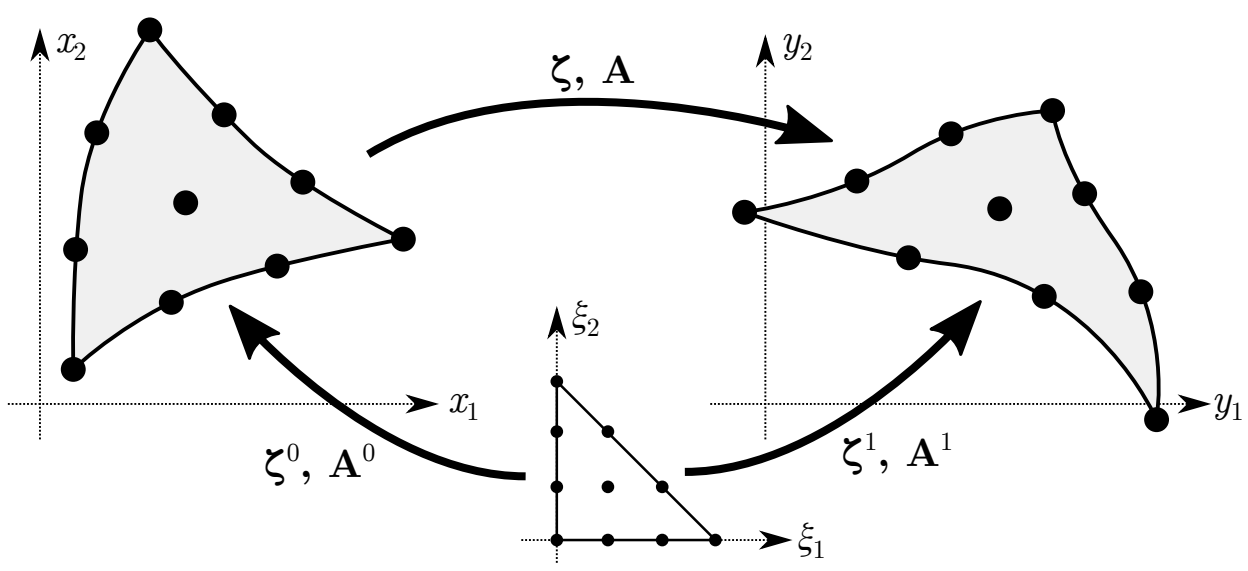

onde $\boldsymbol{\xi}$ é o vetor de coordenadas adimensionais, e $\varphi^{\alpha}$ é a função de forma associada ao nó $\alpha$, definida no elemento finito de coordenadas adimensionais. Dessa forma, calcula-se o 
gradiente da função mudança de configuração (2.1) por

$$
\mathbf{A}=\nabla_{\mathbf{x}}\left(\boldsymbol{\zeta}^{1} \circ\left(\boldsymbol{\zeta}^{0}\right)^{-1}\right)=\mathbf{A}^{1}\left(\mathbf{A}^{0}\right)^{-1}
$$

onde

$$
\begin{aligned}
& A_{i j}^{0}=\zeta_{i, j}^{0}=\frac{\partial \varphi^{\alpha}}{\partial \xi_{j}} x_{i}^{\alpha}, \quad \mathrm{e} \\
& A_{i j}^{1}=\zeta_{i, j}^{1}=\frac{\partial \varphi^{\alpha}}{\partial \xi_{j}} y_{i}^{\alpha},
\end{aligned}
$$

a partir do qual são obtidos o Jacobiano (2.13), o alongamento à direita de Cauchy-Green (2.3) e a deformação de Green-Lagrange (2.5), bem como o tensor das tensões de PiolaKirchhoff de segunda espécie, $\mathbf{S}$, calculado por meio da lei constitutiva do material. Para a posterior aplicação no processo de integração numérica, calcula-se também o Jacobiano da configuração inicial:

$$
J^{0}=\operatorname{det}\left(\mathbf{A}^{0}\right) .
$$

Aplicando o princípio da energia mecânica estacionária (2.37) no MEF posicional, tem-se para cada nó $\alpha$ da estrutura discretizada a seguinte equação de equilíbrio:

$$
\frac{\partial \Pi}{\partial \mathbf{y}^{\alpha}}=\mathbf{f}_{i n t}^{\alpha}+\mathbf{f}_{e x t}^{\alpha}+\mathbf{f}_{i n e r}^{\alpha}=\mathbf{0}
$$

onde os vetores

$$
\begin{aligned}
\mathbf{f}_{i n t}^{\alpha} & =\frac{\partial \Pi_{d e f}}{\partial \mathbf{y}^{\alpha}}, \\
\mathbf{f}_{e x t}^{\alpha} & =\frac{\partial \Pi_{e x t}}{\partial \mathbf{y}^{\alpha}}, \quad \mathrm{e} \\
\mathbf{f}_{i n e r}^{\alpha} & =\frac{\partial \Pi_{c i n}}{\partial \mathbf{y}^{\alpha}}
\end{aligned}
$$

representam, respectivamente, a força interna, externa e inercial do nó $\alpha$. A equação (3.7) representa a forma semi-discreta (apenas discretização espacial) do problema.

\subsection{Integração temporal pelo método de Newmark- $\beta$}

Para a resolução do problema dinâmico, algumas grandezas como velocidade $(\dot{\mathbf{y}})$ e

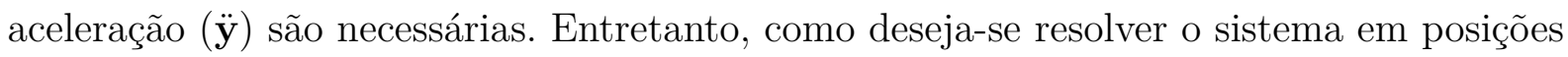
$(\mathbf{y})$, é preciso adotar um algoritmo de integração temporal que permita escrever as variáveis dinâmicas em termos das posições no passo atual e de variáveis conhecidas no passo anterior. Nesse sentido, adota-se o algoritmo de Newmark- $\beta$, que parte das aproximações

$$
\begin{gathered}
\mathbf{y}_{s+1}=\mathbf{y}_{s}+\dot{\mathbf{y}}_{s} \Delta t+\left[(1-2 \beta) \ddot{\mathbf{y}}_{s}+2 \beta \ddot{\mathbf{y}}_{s+1}\right] \frac{\Delta t^{2}}{2}, \quad \mathrm{e} \\
\dot{\mathbf{y}}_{s+1}=\dot{\mathbf{y}}_{s}+(1-\gamma) \Delta t \ddot{\mathbf{y}}_{s}+\gamma \Delta t \ddot{\mathbf{y}}_{s+1},
\end{gathered}
$$

onde $\beta$ e $\gamma$ são os parâmetros de Newmark, os índices $s+1$ e $s$ referem-se, respectivamente, aos valores da variável no passo atual e anterior, e $\Delta t$ é o intervalo de tempo ocorrido entre os passos. 
O método de Newmark- $\beta$ é um integrador temporal generalizado cujos valores especificados para $\beta$ e $\gamma$ permitem realizar tanto integração explícita como implícita (incondicionalmente estável) e atingir convergência de primeira ou de segunda ordem, o que faz que este seja amplamente empregado (NEWMARK, 1959; BAKER, 1976). Os parâmetros mais utilizados na literatura são $\beta=1 / 4$ e $\gamma=1 / 2$, resultando em integração de segunda ordem incondicionalmente estável. Nesse caso as aproximações recaem na regra do trapézio, isto é, considera-se um valor intermediário de aceleração para representar as variações de posição e velocidade. Tais parâmetros são adequados para a maioria das aplicações, com algumas exceções, como problemas de impacto, onde as mudanças bruscas de acelerações podem provocar instabilidade numérica, dificultando a convergência.

Rearranjando as equações (3.11) e (3.12), pode-se escrever as velocidades e acelerações no passo atual como

$$
\begin{gathered}
\dot{\mathbf{y}}_{s+1}=\frac{\gamma}{\beta \Delta t} \mathbf{y}_{s+1}+\mathbf{r}_{s}-\gamma \Delta t \mathbf{q}_{s}, \quad \mathrm{e} \\
\ddot{\mathbf{y}}_{s+1}=\frac{1}{\beta \Delta t^{2}} \mathbf{y}_{s+1}-\mathbf{q}_{s},
\end{gathered}
$$

onde $\mathbf{r}_{s}$ e $\mathbf{q}_{s}$ são grandezas que dependem apenas das variáveis no passo anterior:

$$
\begin{gathered}
\mathbf{r}_{s}=\dot{\mathbf{y}}_{s}+\Delta t(1-\gamma) \ddot{\mathbf{y}}_{s}, \quad \mathrm{e} \\
\mathbf{q}_{s}=\frac{1}{\beta \Delta t^{2}} \mathbf{y}_{s}+\frac{1}{\beta \Delta t} \dot{\mathbf{y}}_{s}+\left(\frac{1}{2 \beta}-1\right) \ddot{\mathbf{y}}_{s} .
\end{gathered}
$$

\subsection{Método de Newton-Raphson}

Suponha que as variáveis nodais $\mathbf{x}^{\alpha}, \mathbf{y}^{\alpha}, \dot{\mathbf{y}}^{\alpha}, \ddot{\mathbf{y}}^{\alpha}, \mathbf{f}_{\text {int }}^{\alpha}, \mathbf{f}_{\text {ext }}^{\alpha}, \mathbf{f}_{\text {iner }}^{\alpha}, \mathbf{q}_{s}$ e $\mathbf{r}_{s}$ sejam arranjadas, respectivamente, em vetores ${ }^{1}\{\mathbf{x}\},\{\mathbf{y}\},\{\dot{\mathbf{y}}\},\{\ddot{\mathbf{y}}\},\{\mathbf{f}\}^{\text {int }},\{\mathbf{f}\}^{\text {ext }},\{\mathbf{f}\}^{\text {iner }},\{\mathbf{q}\}_{s}$ e $\{\mathbf{r}\}_{s}$ de dimensões $N \cdot n_{\text {nós }}$, onde $N$ é o número de dimensões do problema (no caso, 2) e $n_{\text {nós }}$ é o número de nós da malha, a partir da seguinte incidência cinemática:

$$
\{(\bullet)\}_{N(\alpha-1)+i}=(\bullet)_{i}^{\alpha} .
$$

Sendo assim, o sistema não-linear dado por (3.7) pode ser arranjado em um vetor resíduo $\{\mathbf{g}\}$, escrito como:

$$
\{\mathbf{g}\}=\{\mathbf{f}\}^{\text {int }}+\{\mathbf{f}\}^{\text {ext }}+\{\mathbf{f}\}^{\text {iner }}=\{\mathbf{0}\} .
$$

Seja $\{\mathbf{y}\}^{t}$ uma configuração "tentativa" qualquer de posições e denote por $(\bullet)^{t}$ o valor da grandeza genérica $(\bullet)$ aplicada em $\{\mathbf{y}\}^{t}$. Então, expandindo $\{\mathbf{g}\}$ em série de Taylor, e desprezando-se os termos de ordem 2 ou superior, temos

$$
\{\mathbf{g}\}^{t}=-[\mathbf{H}]^{t}\{\Delta \mathbf{y}\}
$$

onde $[\mathbf{H}]=\partial\{\mathbf{g}\} / \partial\{\mathbf{y}\}$ é denominada Matriz Hessiana, ou rigidez tangente, e $\{\Delta \mathbf{y}\}=$ $\{\mathbf{y}\}-\{\mathbf{y}\}^{t}$.

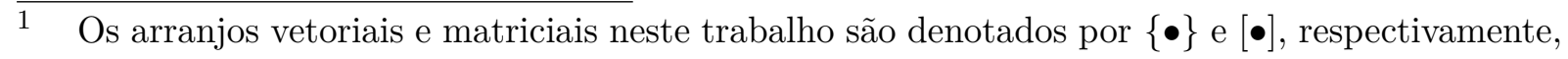
de forma que esses possam ser diferenciados dos vetores e matrizes originais. 
Inicialmente, o vetor $\{\mathbf{y}\}^{t}$ pode assumir os valores do passo anterior ou $\{\mathbf{x}\}$, caso seja o primeiro passo. A partir desse, calcula-se o vetor resíduo tentativa $\{\mathbf{g}\}^{t}$, a hessiana $[\mathbf{H}]^{t}$, e resolve-se o sistema linear dado pela equação (3.19), de onde obtem-se a correção $\{\Delta \mathbf{y}\}$. Essa correção é então somada em $\{\mathbf{y}\}^{t}$, e repete-se o procedimento até que um critério de convergência seja atendido. Tal critério pode ser dado, por exemplo, por

$$
\text { erro }=\frac{\|\{\Delta \mathbf{y}\}\|}{\|\{\mathbf{x}\}\|} \leq \text { tol, }
$$

onde $\|(\bullet)\|$ denota a norma de $(\bullet)$ e tol é um número positivo suficientemente pequeno. Neste trabalho, adota-se tol $=10^{-6}$.

\subsection{Forças internas}

Aplicando a equação (2.50) em (3.8), pode-se determinar o valor nodal equivalente de força interna do nó $\alpha$ pela expressão:

$$
\mathbf{f}_{i n t}^{\alpha}=\int_{\Omega^{0}} \mathbf{S}: \frac{\partial \mathbf{E}}{\partial \mathbf{y}^{\alpha}} d V^{0}
$$

onde, partindo das equações (2.5), (2.3) e (3.3), calcula-se

$$
\frac{\partial E_{i j}}{\partial y_{k}^{\alpha}}=\frac{1}{2}\left(A^{0}\right)_{l i}^{-1} \frac{\partial A_{m l}^{1}}{\partial y_{k}^{\alpha}} A_{m n}^{1}\left(A^{0}\right)_{n j}^{-1}+\frac{1}{2}\left(A^{0}\right)_{l i}^{-1} A_{m l}^{1} \frac{\partial A_{m n}^{1}}{\partial y_{k}^{\alpha}}\left(A^{0}\right)_{n j}^{-1},
$$

e, partindo da equação (3.5),

$$
\frac{\partial A_{i j}^{1}}{\partial y_{k}^{\alpha}}=\frac{\partial \varphi^{\alpha}}{\partial \xi_{j}} \delta_{i k}
$$

onde $\delta$ é a função delta de Kronecker, dada pela equação (A.13). A integral em (3.21) é calculada sobre todos os elementos que contêm o nó $\alpha$, por meio de integração numérica, onde no presente trabalho utiliza-se a quadratura dada por Dunavant (1985).

\subsection{Forças externas}

Aplicando (2.48) em (3.9) e usando a interpolação $\mathbf{y}=\varphi^{\alpha} \mathbf{y}^{\alpha}$, pode-se escrever a força externa associada a cada nó $\alpha$ como:

$$
\mathbf{f}_{e x t}^{\alpha}=-\int_{\Omega^{0}} \varphi^{\alpha} \mathbf{b} d V^{0}-\int_{\Gamma^{0}} \varphi^{\alpha} \mathbf{p} d S^{0}
$$

onde, a fim de reduzir a notação, os vetores de carregamento $\mathbf{b}^{0}$ e $\mathbf{p}^{0}$ foram representados simplesmente por $\mathbf{b}$ e $\mathbf{p}$, e subentende-se que esses são definidos na configuração inicial.

Caso o problema apresente cargas pontuais, essas devem ser somadas diretamente na equação (3.24). Neste trabalho, foram considerados apenas casos onde $\mathbf{p}$ e $\mathbf{b}$ são constantes ao longo do elemento. Portanto, a integral (3.24), resolvida também por integração numérica, é polinomial da ordem de $\varphi^{\alpha}$, e deve ser resolvida sobre todos os elementos que contêm o nó $\alpha$. Caso se deseje incluir forças variáveis, pode-se escrever as expressões para $\mathbf{p}$ e b nas integrais, ou interpolar os carregamentos por valores nodais, 
isto é, $\mathbf{p}=\varphi^{\beta} \mathbf{p}^{\beta}$ e $\mathbf{b}=\varphi^{\beta} \mathbf{b}^{\beta}$, de forma que a equação (3.24) se torne:

$$
\mathbf{f}_{e x t}^{\alpha}=-\int_{\Omega^{0}} \varphi^{\alpha} \varphi^{\beta} \mathbf{p}^{\beta} d V^{0}-\int_{\Gamma^{0}} \varphi^{\alpha} \varphi^{\beta} \mathbf{b}^{\beta} d S^{0} .
$$

\subsection{Forças inerciais}

Aplicando (2.46) em (3.10) e usando as interpolações $\mathbf{y}=\varphi^{\alpha} \mathbf{y}^{\alpha}$ e $\ddot{\mathbf{y}}=\varphi^{\alpha} \ddot{\mathbf{y}}^{\alpha}$, a força inercial associada a cada nó $\alpha$ é dada por:

$$
\mathbf{f}_{\text {iner }}^{\alpha}=\int_{\Omega^{0}} \rho_{0} \varphi^{\alpha} \varphi^{\beta} \ddot{\mathbf{y}}^{\beta} d V^{0}
$$

isto é,

$$
\{\mathbf{f}\}^{\text {iner }}=[\mathbf{M}]\{\ddot{\mathbf{y}}\},
$$

onde $[\mathbf{M}]$ é denominada matriz de massa, com dimensões $\left(N \cdot n_{\text {nós }}\right) \times\left(N \cdot n_{\text {nós }}\right)$. Para cada conjunto de nós $\alpha, \beta$ da malha e direção $i$, corresponde-se um termo da linha $m=N(\alpha-1)+i$ e coluna $n=N(\beta-1)+i$ da matriz $[\mathbf{M}]$, dado pela expressão

$$
[M]_{m n}=\int_{\Omega^{0}} \rho_{0} \varphi^{\alpha} \varphi^{\beta} d V^{0}
$$

onde a integral é calculada sobre todos os elementos que contêm os nós $\alpha$ e $\beta$. Para facilitar a visualização, apresenta-se a matriz de massa de um sólido bidimensional com apenas um elemento de 3 nós:

$$
[\mathbf{M}]=\int_{\Omega^{0}} \rho_{0}\left[\begin{array}{cccccc}
\varphi^{1} \varphi^{1} & 0 & \varphi^{1} \varphi^{2} & 0 & \varphi^{1} \varphi^{3} & 0 \\
0 & \varphi^{1} \varphi^{1} & 0 & \varphi^{1} \varphi^{2} & 0 & \varphi^{1} \varphi^{3} \\
\varphi^{2} \varphi^{1} & 0 & \varphi^{2} \varphi^{2} & 0 & \varphi^{2} \varphi^{3} & 0 \\
0 & \varphi^{2} \varphi^{1} & 0 & \varphi^{2} \varphi^{2} & 0 & \varphi^{2} \varphi^{3} \\
\varphi^{3} \varphi^{1} & 0 & \varphi^{3} \varphi^{2} & 0 & \varphi^{3} \varphi^{3} & 0 \\
0 & \varphi^{3} \varphi^{1} & 0 & \varphi^{3} \varphi^{2} & 0 & \varphi^{3} \varphi^{3}
\end{array}\right] d V^{0}
$$

Adicionalmente, caso se deseje incluir amortecimento de Rayleigh:

$$
\{\mathbf{f}\}^{\text {iner }}=[\mathbf{M}]\{\ddot{\mathbf{y}}\}+[\mathbf{C}]\{\dot{\mathbf{y}}\}
$$

onde $[\mathbf{C}]$ é a matriz de amortecimento, que pode ser adotada, por exemplo, como combinação linear das matrizes de massa e de rigidez.

Aplicando as equações (3.11) e (3.12) do algoritmo de Newmark- $\beta$ nos vetores $\{\ddot{\mathbf{y}}\}$ e $\{\dot{\mathbf{y}}\}$ da equação (3.30), pode-se expressa-la na forma:

$$
\{\mathbf{f}\}^{\text {iner }}=\frac{1}{\beta \Delta t^{2}}[\mathbf{M}]\{\mathbf{y}\}-[\mathbf{M}]\{\mathbf{q}\}_{s}+\frac{\gamma}{\beta \Delta t}[\mathbf{C}]\{\mathbf{y}\}+[\mathbf{C}]\{\mathbf{r}\}_{s}-\gamma \Delta t[\mathbf{C}]\{\mathbf{q}\}_{s},
$$

lembrando que $\{\mathbf{q}\}_{s}$ e $\{\mathbf{r}\}_{s}$ dependem apenas das posições, velocidades e acelerações do passo anterior, que são conhecidas.

Caso seja o primeiro passo e os valores iniciais de força interna $\{\mathbf{f}\}_{0}^{\text {int }}$, força externa $\{\mathbf{f}\}_{0}^{e x t}$ e velocidade $\{\dot{\mathbf{y}}\}_{0}$ sejam dados, a aceleração inicial $\{\ddot{\mathbf{y}}\}_{0}$ pode ser calculada pelo sistema linear

$$
[\mathbf{M}]\{\ddot{\mathbf{y}}\}_{0}=-\{\mathbf{f}\}_{0}^{\text {int }}-\{\mathbf{f}\}_{0}^{e x t}-[\mathbf{C}]\{\dot{\mathbf{y}}\}_{0}
$$




\subsection{Matriz Hessiana}

Considerando-se as forças externas conservativas, isto é, independentes de mudanças de posições, resulta a seguinte expressão para a matriz hessiana:

$$
[\mathbf{H}]=\frac{\partial\{\mathbf{g}\}}{\partial\{\mathbf{y}\}}={\frac{\partial\{\mathbf{f}\}^{\text {int }}}{\partial\{\mathbf{y}\}}+\frac{\partial\{\mathbf{f}\}^{\text {iner }}}{\partial\{\mathbf{y}\}}}^{\text {. }}
$$

onde, das equações (3.31) e (3.21), resultam

$$
\begin{gathered}
\frac{\partial\{\mathbf{f}\}^{\text {iner }}}{\partial\{\mathbf{y}\}}=\frac{1}{\beta \Delta t^{2}}[\mathbf{M}]+\frac{\gamma}{\beta \Delta t}[\mathbf{C}], \quad \mathrm{e} \\
\frac{\partial \mathbf{f}_{i n t}^{\alpha}}{\partial \mathbf{y}^{\beta}}=\int_{\Omega^{0}}\left(\frac{\partial \mathbf{S}}{\partial \mathbf{y}^{\beta}}: \frac{\partial \mathbf{E}}{\partial \mathbf{y}^{\alpha}}+\mathbf{S}: \frac{\partial^{2} \mathbf{E}}{\partial \mathbf{y}^{\alpha} \partial \mathbf{y}^{\beta}}\right) d V^{0}
\end{gathered}
$$

e, da equação (3.22),

$$
\frac{\partial^{2} E_{i j}}{\partial y_{i}^{\alpha} \partial y_{j}^{\beta}}=\frac{1}{2}\left(A^{0}\right)_{l i}^{-1} \frac{\partial A_{m l}^{1}}{\partial y_{i}^{\alpha}} \frac{\partial A_{m n}^{1}}{\partial y_{j}^{\beta}}\left(A^{0}\right)_{n j}^{-1}+\frac{1}{2}\left(A^{0}\right)_{l i}^{-1} \frac{\partial A_{m l}^{1}}{\partial y_{j}^{\beta}} \frac{\partial A_{m n}^{1}}{\partial y_{i}^{\alpha}}\left(A^{0}\right)_{n j}^{-1},
$$

lembrando que $\partial \mathbf{A}^{1} / \partial \mathbf{y}$ é dado na Equação (3.23). O valor de $\partial \mathbf{E} / \partial \mathbf{y}$, necessário na equação (3.35), também já apresentado em (3.22). Além disso,

$$
\frac{\partial \mathbf{S}}{\partial \mathbf{y}}=\frac{\partial \mathbf{S}}{\partial \mathbf{E}}: \frac{\partial \mathbf{E}}{\partial \mathbf{y}}
$$

onde $\partial \mathbf{S} / \partial \mathbf{E}$, já abordado anteriormente, é o operador tangente consistente da lei constitutiva, denotado, no caso hiperelástico, por $\mathfrak{C}^{e}$.

O valor obtido em (3.35) é aplicado a (3.33) utilizando a seguinte incidência cinemática:

$$
\frac{\partial\{f\}_{N(\alpha-1)+i}^{\text {int }}}{\partial\{y\}_{N(\beta-1)+j}}=\frac{\partial\left(f_{i n t}^{\alpha}\right)_{i}}{\partial y_{j}^{\beta}}
$$

\subsection{Implementação computacional}

No algoritmo 1 ilustra-se o processo descrito por meio de um esboço do código computacional desenvolvido, sendo abordados alguns detalhes não discutidos previamente, como por exemplo imposição de posições prescritas.

O algoritmo é implementado em linguagem Fortran 95. O loop iniciado na linha 12 é paralelizado, isto é, executado em vários núcleos de processamento simultaneamente, por meio da ferramenta OpenMP (DAGUM; MENON, 1998), o que ajudou a diminuir consideravelmente o tempo de execução do programa.

Vale ser dito que, embora o código tenha sido desenvolvido e apresentado para problemas dinâmicos, esse também pode ser utilizado para realizar análises puramente estáticas, sendo tal limitante definido pelo usuário nos dados de entrada. Nesse caso, o programa irá apenas assumir que $\{\mathbf{f}\}^{\text {iner }}=\{\mathbf{0}\}, \partial\{\mathbf{f}\}^{\text {iner }} / \partial\{\mathbf{y}\}=[\mathbf{0}]$, e ignorar o cálculo das variáveis dinâmicas $[\mathbf{M}],[\mathbf{C}],\{\ddot{\mathbf{y}}\}_{0},\{\mathbf{q}\}_{s},\{\mathbf{r}\}_{s},\{\dot{\mathbf{y}}\}_{s+1}$ e $\{\ddot{\mathbf{y}}\}_{s+1}$ (linhas 3, 5 e 39).

Algumas variáveis calculadas em pontos de integração, tais como deformações 


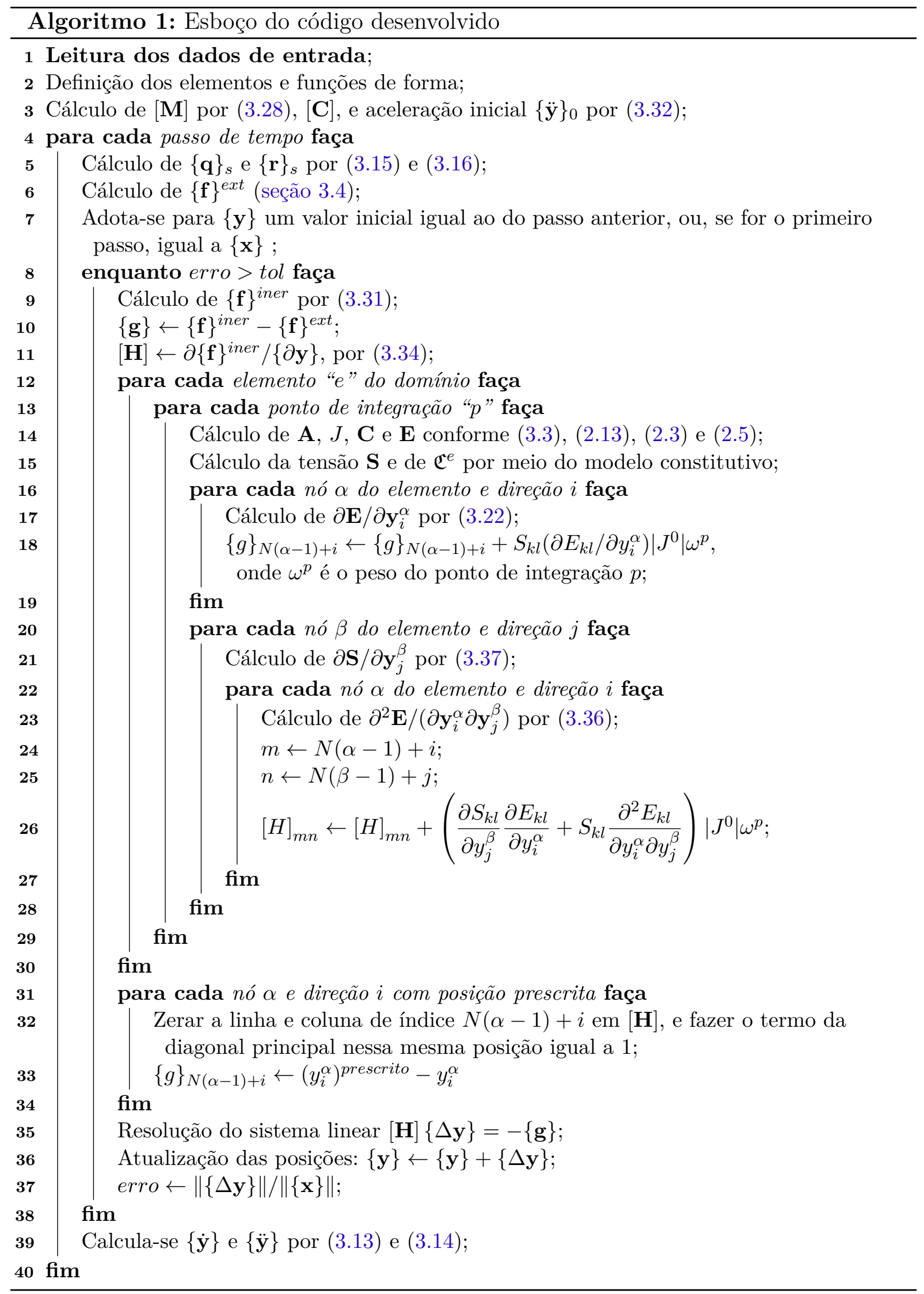

e tensões, precisam ser projetadas aos nós para que possam ser visualizadas em pósprocessadores comuns. Nesse sentido, foi utilizada uma estratégia de interpolação baseada no método dos mínimos quadrados, tal como descrito em Coda (2018). 


\section{Capítulo 4}

\section{Modelo elasto-plástico e visco-plástico}

Em contraste aos modelos elásticos, onde há o retorno à configuração inicial após o descarregamento, modelos constitutivos plásticos são caracterizados por sua natureza dissipativa, apresentando deformações permanentes. Isso ocorre devido ao movimento irreversível de discordâncias, que provocam deslizamentos na estrutura microscópica do material.

Quando a lei constitutiva apresenta características simultaneamente elásticas e plásticas, essa é denominada elasto-plástica, sendo tal nomenclatura utilizada principalmente para modelos independentes do tempo. Quando a parcela plástica depende explicitamente das taxas de deformações às quais o material é sujeito, o modelo é denominado elasto-visco-plástico, ou, simplesmente, visco-plástico.

Nas seções 4.1 e 4.2, são descritos dois modelos elasto-plásticos baseados na energia de dissipação, que diferenciam-se essencialmente pela forma de decomposição adotada para as deformações plásticas e elásticas. O primeiro parte da decomposição de Green-Naghdi (ou aditiva), sendo válido somente para o caso de pequenas deformações. Já o segundo, bem aceito no regime de grandes deformações, parte da decomposição de Kröner-Lee (ou multiplicativa), sendo baseado nos trabalhos de Svendsen (1998), Dettmer e Reese (2004) e Pascon e Coda (2013a). Esse último é generalizado ainda para o caso visco-plástico na seção 4.3, onde se utiliza o clássico modelo de Perzyna.

\subsection{Modelo elasto-plástico em pequenas deformações}

Com o intuito de facilitar a visualização de certos conceitos básicos referentes à elasto-plasticidade, apresenta-se nesta seção um modelo simplificado baseado na decomposição aditiva do tensor de deformações de Green-Lagrange. Tal decomposição permite uma notável redução nos cálculos quando comparada à multiplicativa, porém, apresenta determinadas incoerências que limitam seu uso, tradicionalmente, ao regime de pequenas deformações. 


\subsubsection{Concepção unidimensional}

No modelo elasto-plástico, o limite entre o regime puramente elástico e o início do escoamento deve ser regido por um critério de plastificação (ou de escoamento). Para exemplificar, considera-se uma barra de treliça submetida à uma deformação linear $\varepsilon$, que provoca uma tensão uniaxial $\sigma$. Então, o escoamento tem início quando o valor de $\sigma$ atinge uma tensão limite de escoamento, $\sigma_{Y}$. No modelo elasto-plástico perfeito, o valor de $\sigma_{Y}$ é constante, e toda deformação ocorrida durante o escoamento é considerada plástica, isto é, permanece mesmo após a barra ser descarregada. A Figura 10a ilustra uma possível relação tensão-deformação ocorrida nesse caso. Assim, pode-se estabelecer a decomposição aditiva da deformação:

$$
\varepsilon=\varepsilon^{e}+\varepsilon^{p}
$$

onde $\varepsilon^{e}$ é a parcela elástica, que, nesse caso, relaciona-se linearmente com a tensão $\sigma$, enquanto $\varepsilon^{p}$ é a parcela plástica da deformação.

Em geral, podemos escrever o critério de plastificação por meio de uma função $\Phi$, denominada aqui função critério de plastificação. No caso elasto-plástico perfeito, temos

$$
\Phi(\sigma)=|\sigma|-\sigma_{Y} \leq 0 .
$$

Quando as condições de plastificação mudam ao longo da análise, diz-se que há encruamento. No caso com encruamento isotrópico, temos

$$
\Phi(\sigma, \kappa)=|\sigma|-\sigma_{\kappa}(\kappa) \leq 0
$$

onde $\kappa$ é denominado parâmetro de encruamento isotrópico e $\sigma_{\kappa}(\kappa)$ é a função de escoamento. Nesse sentido, um modelo comumente adotado parte de

$$
\dot{\kappa}=\left|\dot{\varepsilon}^{p}\right| \quad \text { e } \quad \sigma_{\kappa}=\sigma_{Y}+H \kappa,
$$

onde $\sigma_{Y}$ e $H$ são constantes do material. Esse caso é ilustrado na Figura 10b, onde nota-se que o escoamento, iniciado a partir do momento que $|\sigma|$ alcança $\sigma_{Y}$, é composto não apenas por deformações plásticas, mas pela composição entre deformações elásticas e plásticas, caracterizando o regime como elasto-plástico.

Figura 10 - Relação tensão-deformação em modelos elasto-plásticos unidimensionais, onde (I) representa a etapa de carregamento elástico, (II) a etapa a partir do escoamento, e (III) o descarregamento

(a) Modelo elasto-plástico perfeito

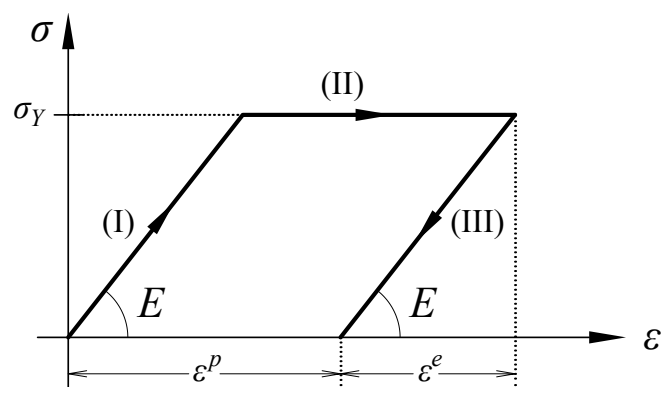

(b) Modelo com encruamento isotrópico

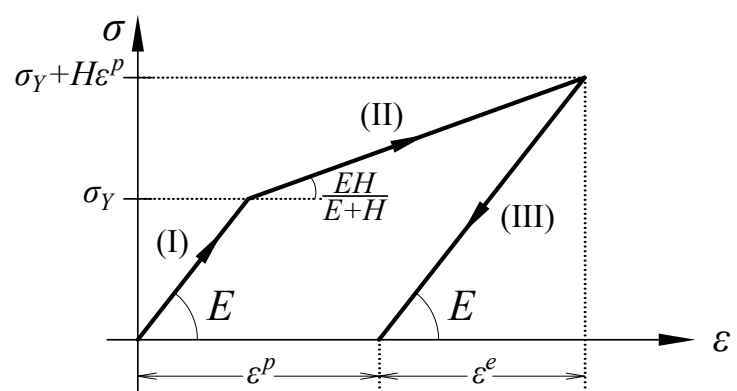




\subsubsection{Decomposição de Green-Naghdi}

Expandindo-se a expressão (4.1) para um caso com mais dimensões, obtém-se:

$$
\mathbf{E}=\mathbf{E}^{e}+\mathbf{E}^{p}
$$

onde $\mathbf{E}^{e}$ e $\mathbf{E}^{p}$ são, respectivamente, as parcelas elástica e plástica do tensor de deformação de Green-Lagrange. A Equação (4.5) é chamada de decomposição aditiva, ou decomposição de Green-Naghdi. Conforme observado no trabalho de Eterovic e Bathe (1991), tal decomposição faz com que a resposta elástica seja, de maneira indesejável, dependente das deformações inelásticas anteriores. Portanto, de maneira geral, essa não é bem aceita na literatura, especialmente no contexto de grandes deformações.

Como consequência direta da Equação (4.5), a taxa de deformação de Green também pode ser decomposta aditivamente, isto é:

$$
\dot{\mathbf{E}}=\dot{\mathbf{E}}^{e}+\dot{\mathbf{E}}^{p}
$$

Adicionalmente, a fim de representar os efeitos do encruamento, a parcela de deformação plástica de Green-Lagrange e sua respectiva taxa podem ser escritas por meio de uma segunda decomposição:

$$
\begin{aligned}
\mathbf{E}^{p} & =\mathbf{E}_{e}^{p}+\mathbf{E}_{i}^{p}, \quad \mathrm{e} \\
\dot{\mathbf{E}}^{p} & =\dot{\mathbf{E}}_{e}^{p}+\dot{\mathbf{E}}_{i}^{p},
\end{aligned}
$$

onde $\mathbf{E}_{e}^{p}$ representa as deformações plásticas elásticas, induzidas por discordâncias, e $\mathbf{E}_{i}^{p}$ as deformações plásticas irreversíveis devidas a escorregamentos nos cristais (LION, 2000). Na Figura 11 é apresentado o modelo reológico adotado, onde é possível observar cada parcela de deformação apresentada.

Figura 11 - Modelo reológico de material elasto-plástico com encruamento cinemático, adaptado de Vladimirov, Pietryga e Reese (2007)

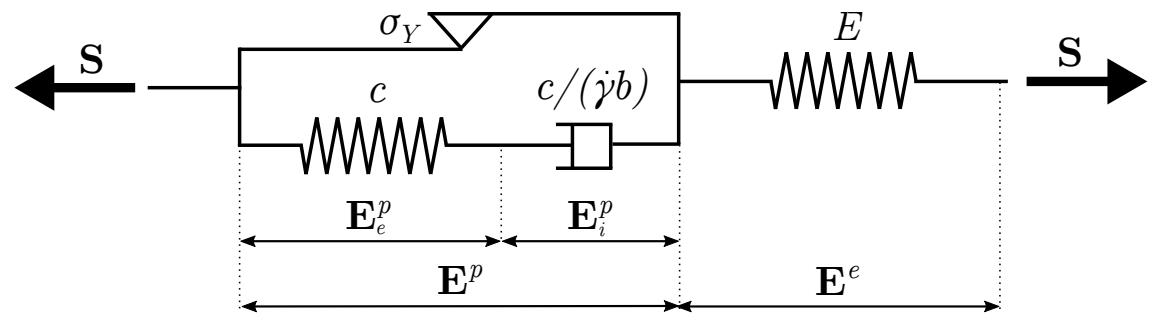

\subsubsection{Energia, tensão e dissipação}

Baseado em modelos reológicos, é possível decompor a energia livre de Helmholtz de forma aditiva em uma parcela elástica $\Psi^{e}$, que depende apenas de $\mathbf{E}^{e}$, e outra parcela plástica $\Psi^{p}$. Essa última, por sua vez, possui uma parcela cinemática, dependente de $\mathbf{E}_{e}^{p}$, e uma isotrópica, dependente do parâmetro de encruamento $\kappa$. Dessa forma, escreve-se:

$$
\Psi\left(\mathbf{E}^{e}, \mathbf{E}_{e}^{p}, \kappa\right)=\Psi^{e}\left(\mathbf{E}^{e}\right)+\Psi_{c i n}^{p}\left(\mathbf{E}_{e}^{p}\right)+\Psi_{i s o}^{p}(\kappa) .
$$


Com base nisso, deriva-se a tensão elástica, a tensão de retorno (back stress), e a tensão de escoamento, respectivamente, pelas expressões (COLEMAN; NOLL, 1963):

$$
\mathbf{S}^{e}=\frac{\partial \Psi^{e}}{\partial \mathbf{E}^{e}}, \quad \mathbf{X}=\frac{\partial \Psi_{c i n}^{p}}{\partial \mathbf{E}_{e}^{p}} \quad \text { e } \quad \sigma_{\kappa}=\frac{\partial \Psi_{i s o}^{p}}{\partial \kappa}
$$

As parcelas $\Psi^{e}$ e $\Psi_{\text {cin }}^{p}$ devem ser tratadas como leis hiperelásticas em função de $\mathbf{E}^{e}$ e $\mathbf{E}_{e}^{p}$ respectivamente, e podem ser tomadas com base nos modelos discutidos na seção 2.5. Para a lei de Saint Vennant-Kirchhoff, por exemplo, temos:

$$
\Psi^{e}=G \operatorname{tr}\left(\mathbf{E}^{e} \cdot \mathbf{E}^{e}\right)+\frac{\lambda}{2} \operatorname{tr}\left(\mathbf{E}^{e}\right)^{2} \quad \text { e } \quad \Psi_{e}^{p}=\frac{c}{2} \operatorname{tr}\left(\mathbf{E}_{e}^{p} \cdot \mathbf{E}_{e}^{p}\right),
$$

de onde resultam

$$
\mathbf{S}^{e}=2 G \mathbf{E}^{e}+\lambda \operatorname{tr}\left(\mathbf{E}^{e}\right) \mathbf{I} \quad \text { e } \quad \mathbf{X}=c \mathbf{E}_{e}^{p}
$$

onde $\lambda$ é a constante de Lamé, $G$ é o módulo de elasticidade transversal, e $c$ é o coeficiente de rigidez do encruamento cinemático. As leis para $\sigma_{\kappa}$ serão discutidas posteriormente.

Para calcular a energia de dissipação interna, utiliza-se a segunda lei da termodinâmica, expressa pela inequação de Clausius-Duhem (HOLZAPFEL, 2000):

$$
d_{\text {int }}=\mathbf{S}: \dot{\mathbf{E}}-\dot{\Psi} \geq 0
$$

de onde, aplicando (4.6) e (4.8), resulta:

$$
\begin{aligned}
d_{i n t} & =\mathbf{S}: \dot{\mathbf{E}}-\left(\frac{\partial \Psi^{e}}{\partial \mathbf{E}^{e}}: \dot{\mathbf{E}}^{e}+\frac{\partial \Psi_{c i n}^{p}}{\partial \mathbf{E}_{e}^{p}}: \dot{\mathbf{E}}_{e}^{p}+\frac{\partial \Psi_{i s o}^{p}}{\partial \kappa}: \dot{\kappa}\right) \\
& =\mathbf{S}: \dot{\mathbf{E}}-\mathbf{S}^{e}:\left(\dot{\mathbf{E}}-\dot{\mathbf{E}}^{p}\right)-\mathbf{X}:\left(\dot{\mathbf{E}}^{p}-\dot{\mathbf{E}}_{i}^{p}\right)-\sigma_{\kappa} \dot{\kappa} \\
& =\left(\mathbf{S}-\mathbf{S}^{e}\right): \dot{\mathbf{E}}+\left(\mathbf{S}^{e}-\mathbf{X}\right): \dot{\mathbf{E}}^{p}+\mathbf{X}: \dot{\mathbf{E}}_{i}^{p}-\sigma_{\kappa} \dot{\kappa} \geq 0 .
\end{aligned}
$$

Para garantir a não-negatividade da energia de dissipação para valores arbitrários de $\dot{\mathbf{E}}$, define-se a tensão de Piola-Kirchhoff de segunda espécie como (HOLZAPFEL, 2000):

$$
\mathbf{S}=\mathbf{S}^{e},
$$

de forma que a energia de dissipação é dada por:

$$
d_{i n t}=(\mathbf{S}-\mathbf{X}): \dot{\mathbf{E}}^{p}+\mathbf{X}: \dot{\mathbf{E}}_{i}^{p}-\sigma_{\kappa} \dot{\kappa} \geq 0
$$

Diz-se então que $\mathbf{S}-\mathbf{X}$ é uma medida de tensão termodinamicamente conjugada a $\dot{\mathbf{E}}^{p}$ (SVENDSEN, 1998) e, portanto, é utilizada para definir o critério de plastificação do modelo (OTTOSEN; RISTINMAA, 2005).

\subsubsection{Critério de plastificação}

Para o caso em maiores dimensões, há diversas formas de formular o critério de plastificação. Entre os mais conhecidos critérios, podem ser citados os de Mohr-Coulomb, Drucker-Prager, Tresca e von Mises. Neste trabalho opta-se pelo critério de von Mises, que baseia-se na máxima energia de distorção e é amplamente aplicado a mateirais de comportamento dúctil. No contexto da formulação descrita, a função critério de plastificação 
pode ser expressa como:

$$
\Phi\left(\mathbf{S}, \mathbf{X}, \sigma_{\kappa}\right)=\left\|(\mathbf{S}-\mathbf{X})^{D}\right\|-\sqrt{\frac{2}{3}} \sigma_{\kappa} \leq 0,
$$

onde o subscrito $D$ representa a parcela desviadora do tensor (seção 2.4) e a constante $\sqrt{2 / 3}$ tem o propósito de normalizar o critério de forma que esse seja equivalente ao caso unidimensional.

Representando a Equação (4.17) no espaço das tensões principais, temos a chamada superfície de plastificação, que delimita $\mathbf{S}$ a um cilindro no espaço das tensões principais $\left(\mathbf{S}_{1}^{p}, \mathbf{S}_{2}^{p}, \mathbf{S}_{3}^{p}\right)$ cujo eixo passa pela tensão de retorno $\mathbf{X}$ e cujo raio é definido por $\sigma_{\kappa}$, conforme ilustrado na Figura 12.

Figura 12 - Superfície de von Mises no espaço das tensões principais considerando encruamento
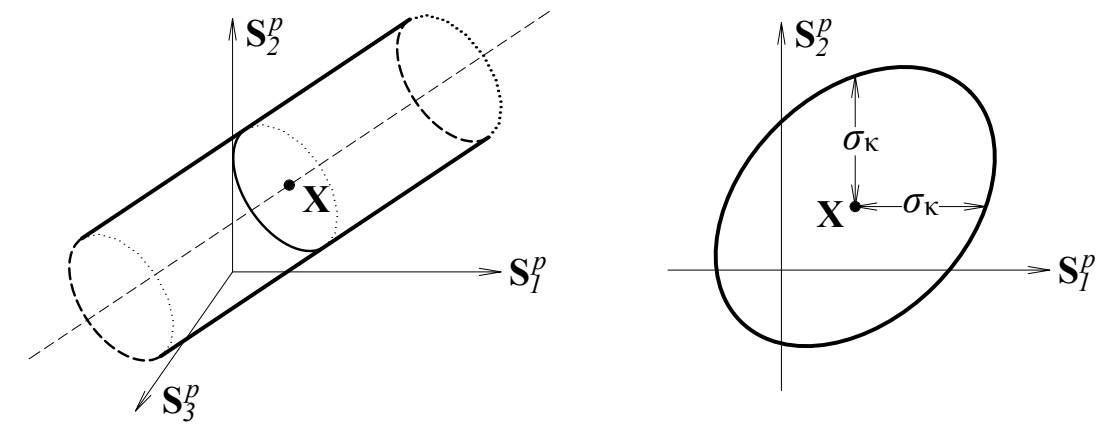

\subsubsection{Encruamento}

Extrapolando o conceito abordado no caso unidimensional, diz-se que há encruamento quando ocorrem alterações na superfície de plastificação ao longo do processo. De acordo com Lemaitre (2001), esse fenômeno pode estar relacionado ao acúmulo de discordâncias (caso isotrópico) ou à presença de tensões residuais internas no nível cristalino (caso cinemático).

No encruamento isotrópico, a superfície é redimensionada, porém não há translação, isso é, seu centro permanece fixo (Figura 13a). Tal fenômeno é controlado pela função $\sigma_{\kappa}$ por meio da variável $\kappa$, e depende do modelo utilizado para o material em estudo, comumente obtido por meio de análises experimentais. Na Tabela 1 alguns modelos são apresentados, sendo o caso mais simples possível aquele no qual a função de escoamento é uma constante, onde diz-se que não há encruamento isotrópico. As leis de Swift e Polinomial, na forma como são apresentadas, foram extraídas do trabalho de Pascon e Coda (2013a), e a lei de Voce do trabalho de Simo e Hughes (2000). Observa-se que, com paramêtros convenientes, as leis Polinomial e de Voce podem retornar o modelo sem encruamento isotrópico. Além disso, a lei polinomial resulta na Equação (4.4) quando $n=1, a_{0}=\sigma_{Y}$ e $a_{1}=H$. Na Tabela 1 , apresentam-se ainda as derivadas de cada lei apresentada, pois essas são utilizadas em futuras aplicações numéricas.

Já no encruamento cinemático, ocorre translação da superfície de plastificação, 
Tabela 1 - Leis de encruamento isotrópico

\begin{tabular}{l|l|l|l}
\hline Lei & Parâmetros & Expressão & Derivada \\
\hline- & $\sigma_{Y}$ & $\sigma_{\kappa}(\kappa)=\sigma_{Y}$ & $\sigma_{\kappa}^{\prime}(\kappa)=0$ \\
\hline Lei de Swift & $k, \varepsilon_{0}$ e $n$ & $\sigma_{\kappa}(\kappa)=k\left(\varepsilon_{0}+\kappa\right)^{n}$ & $\sigma_{\kappa}^{\prime}(\kappa)=k n\left(\varepsilon_{0}+\kappa\right)^{n-1}$ \\
\hline Lei de Voce & $\sigma_{Y}, Q$ e $\beta$ & $\sigma_{\kappa}(\kappa)=\sigma_{Y}+Q\left(1-e^{-\beta \kappa}\right)$ & $\sigma_{\kappa}^{\prime}(\kappa)=\beta Q e^{-\beta \kappa}$ \\
\hline Lei polinomial & $\begin{array}{l}n \text { (inteiro) e } a_{i} \\
(\text { para } i=1, \ldots, n)\end{array}$ & $\sigma_{\kappa}(\kappa)=\sum_{i=0}^{n} a_{i} \kappa^{i}$ & $\sigma_{\kappa}^{\prime}(\kappa)=\sum_{i=1}^{n} i a_{i} \kappa^{i-1}$ \\
\hline
\end{tabular}

porém não há redimensionamento (Figura 13b). Na formulação adotada, esse processo é controlado pela tensão de retorno $\mathbf{X}$. No presente trabalho, para representar o encruamento cinemático utiliza-se a lei de Armstrong-Frederick (ARMSTRONG; FREDERICK, 1966), discutida com detalhes em seções posteriores. Outras leis comumente adotadas podem ser vistas, por exemplo, em Pascon (2012).

O encruamento isotrópico, individualmente, é suficiente para modelar uma grande variedade de exemplos, especialmente os que apresentam carregamento em uma única direção. Entretanto, em casos de carregamentos cíciclos, onde podem ser manifestados os efeitos de Bauschinger e fluência cíclica (ratcheting), a consideração do encruamento cinemático é fundamental, conforme discutido em Vladimirov, Pietryga e Reese (2007).

Figura 13 - Representação dos tipos de encruamento

(a) Encruamento isotrópico

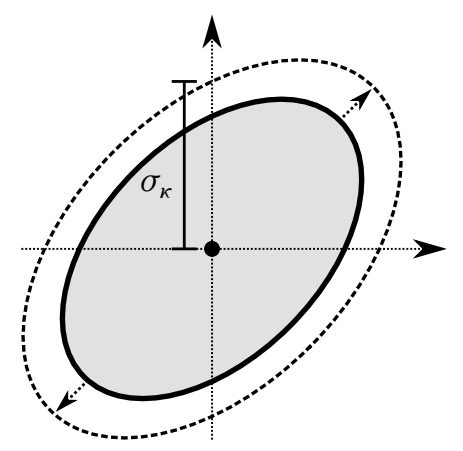

(b) Encruamento cinemático

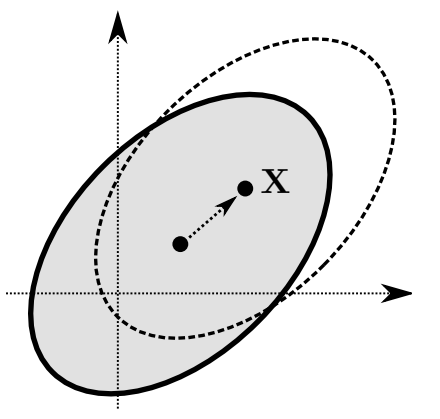

\subsubsection{Leis de evolução}

Pelo princípio da máxima dissipação da energia, deve-se maximizar o valor de $d_{\text {int }}$, isto é, minimizar o valor de $-d_{\text {int }}$, quando restrito à condição de plastificação $\Phi \leq 0$. Para aplicar tal condição, pode-se utilizar o método dos multiplicadores de Lagrange adicionando ao funcional de energia tal condição subsidiária, de forma que o funcional a ser minimizado é dado por:

$$
\ell\left(\mathbf{S}, \mathbf{X}, \sigma_{\kappa}, \dot{\gamma}\right)=-d_{i n t}+\dot{\gamma} \Phi=-(\mathbf{S}-\mathbf{X}): \dot{\mathbf{E}}^{p}-\mathbf{X}: \dot{\mathbf{E}}_{i}^{p}+\sigma_{\kappa} \dot{\kappa}+\dot{\gamma} \Phi .
$$

onde $\dot{\gamma}$ é o multiplicador de Lagrange.

Para que a restrição não seja aplicada nos casos em que $\Phi<0$, utiliza-se uma 
equação adicional, denominada condição de complementaridade (ou de Kuhn-Tucker):

$$
\dot{\gamma} \Phi=0 \text {. }
$$

Dessa forma, caso $\Phi<0$, segue automaticamente que $\dot{\gamma}=0$. Caso contrário, deverá ser calculado o valor de $\dot{\gamma}$ (positivo) tal que $\Phi=0$. As implicações dessa condição serão abordadas com mais detalhes na subseção 4.1.7.

Minimizando $\ell$ com relação a $\mathbf{S}, \sigma_{\kappa}$ e $\mathbf{X}$, temos, respectivamente,

$$
\begin{gathered}
\frac{\partial \ell}{\partial \mathbf{S}}=-\dot{\mathbf{E}}^{p}+\dot{\gamma} \frac{\partial \Phi}{\partial \mathbf{S}}=\mathbf{0} \\
\frac{\partial \ell}{\partial \sigma_{\kappa}}=\dot{\kappa}+\dot{\gamma} \frac{\partial \Phi}{\partial \sigma_{\kappa}}=0, \quad \mathrm{e} \\
\frac{\partial \ell}{\partial \mathbf{X}}=\dot{\mathbf{E}}^{p}-\dot{\mathbf{E}}_{i}^{p}+\dot{\gamma} \frac{\partial \Phi}{\partial \mathbf{X}}=\dot{\mathbf{E}}_{e}^{p}+\dot{\gamma} \frac{\partial \Phi}{\partial \mathbf{X}}=\frac{1}{c} \dot{\mathbf{X}}+\dot{\gamma} \frac{\partial \Phi}{\partial \mathbf{X}}=\mathbf{0},
\end{gathered}
$$

onde na última passagem são aplicadas as equações (4.8) e (4.12). Disso resultam as leis de evolução:

$$
\dot{\mathbf{E}}^{p}=\dot{\gamma} \mathbf{N}^{p}, \quad \dot{\kappa}=\dot{\gamma} n_{\kappa} \quad \text { e } \quad \dot{\mathbf{X}}=\dot{\gamma} \mathbf{N}_{\mathbf{X}}
$$

onde $\mathbf{N}^{p}, n_{\kappa}$ e $\mathbf{N}_{\mathbf{X}}$, responsáveis por definir as direções de evolução, são dados por:

$$
\mathbf{N}^{p}=\frac{\partial \Phi}{\partial \mathbf{S}}, \quad n_{\kappa}=-\frac{\partial \Phi}{\partial \sigma_{\kappa}} \quad \text { e } \quad \mathbf{N}_{\mathbf{X}}=-c \frac{\partial \Phi}{\partial \mathbf{X}} .
$$

Considerando-se agora uma lei de potencial plástico $\Omega$, tal que:

$$
\mathbf{N}^{p}=\frac{\partial \Omega}{\partial \mathbf{S}}, \quad n_{\kappa}=-\frac{\partial \Omega}{\partial \sigma_{\kappa}} \quad \text { e } \quad \mathbf{N}_{\mathbf{X}}=-c \frac{\partial \Omega}{\partial \mathbf{X}},
$$

nota-se que o princípio da máxima dissipação será respeitado apenas se $\Omega=\Phi$, caso no qual a lei é dita associativa. Entretanto, muitos modelos, incluindo o apresentado neste trabalho, utilizam leis não-associativas para representar o comportamento dos materiais. Nesses casos, o princípio da máxima dissipação pode ser substituído pelo princípio da máxima dissipação reduzida, conforme discutido no trabalho de Polizzotto (1998).

A lei de encruamento cinemático de Armstrong-Frederick, no caso de pequenas deformações, deriva do seguinte potencial plástico (DETTMER; REESE, 2004):

$$
\Omega=\Phi+\frac{b}{2 c} \mathbf{X}: \mathbf{X}
$$

onde $b$ é uma constante adimensional dependente do material. Observa-se que, apesar de $\Omega$ ser não-associativa, tem-se $\mathbf{N}^{p}=\partial \Omega / \partial \mathbf{S}=\partial \Phi / \partial \mathbf{S}$. Logo, esse modelo preserva uma característica importante das leis associativas: a ortogonalidade de $\mathbf{N}^{p}$ com relação à superfície de plastificação (ver Figura 14). 
Figura 14 - Representação de $\mathbf{N}^{p}$ no espaço das tensões principais

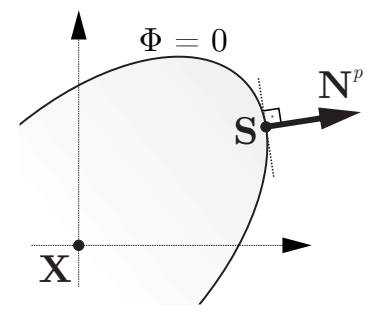

Aplicando (4.26) em (4.25), tem-se:

$$
\left\{\begin{array}{l}
\mathbf{N}^{p}=\frac{\partial \Phi}{\partial \mathbf{S}}=\frac{\partial\left\|(\mathbf{S}-\mathbf{X})^{D}\right\|}{\partial(\mathbf{S}-\mathbf{X})^{D}}: \frac{\partial(\mathbf{S}-\mathbf{X})^{D}}{\partial \mathbf{S}}=\frac{(\mathbf{S}-\mathbf{X})^{D}}{\left\|(\mathbf{S}-\mathbf{X})^{D}\right\|} \\
n_{\kappa}=-\frac{\partial \Omega}{\partial \sigma_{\kappa}}=\sqrt{\frac{2}{3}}, \quad \mathrm{e} \\
\mathbf{N}_{\mathbf{X}}=-c \frac{\partial \Omega}{\partial \mathbf{X}}=-c \frac{\partial \Phi}{\partial \mathbf{X}}-b \mathbf{X}=c \mathbf{N}^{p}-b \mathbf{X}
\end{array}\right.
$$

onde a última passagem é a forma clássica da lei de encruamento cinemático de ArmstrongFrederick.

\subsubsection{Condições de complementaridade e consistência}

Conforme observado na subseção 4.1.6, as leis de evolução das variáveis plásticas são condicionadas pelo valor $\dot{\gamma}$. Sendo assim, em regimes elásticos, deve-se garantir que $\dot{\gamma}$ seja nulo. Por outro lado, na ocorrência de plastificação, $\dot{\gamma}$ deve ser maior que 0. Esse critério é formalizado pela condição de complementaridade (ou de Kuhn-Tucker), e pela condição de consistência, dadas respectivamente por

$$
\begin{aligned}
& \dot{\gamma} \Phi=0, \quad \text { e } \\
& \dot{\gamma} \dot{\Phi}=0,
\end{aligned}
$$

isto é:

- Se $\Phi<0, \dot{\gamma}=0$ (regime elástico);

- Só pode haver evolução das variáveis plásticas $(\dot{\gamma}>0)$ se $\Phi=0$ e $\dot{\Phi}=0$, isto é, se a tensão atual encontra-se na superfície de plastificação e permanece nela;

- Se $\dot{\Phi} \neq 0, \dot{\gamma}=0$. Em particular, se a tensão previamente localizada na superfície de plastificação $(\Phi=0)$ retorna para um ponto onde $\Phi<0$, considera-se que ela retornou ao regime elástico, logo não há evolução das variáveis plásticas.

Em uma aplicação computacional, assume-se inicialmente que $\dot{\gamma}=0$, e realiza-se uma previsão elástica da tensão, a partir da qual calcula-se o valor de $\Phi$. Caso $\Phi<0$, mantêm-se a previsão elástica. Caso contrário, deve-se aplicar uma correção plástica por meio de um algoritmo de retorno, onde se calcula o valor de $\dot{\gamma}$ tal que $\Phi=0$. Tendo em vista que não foi realizado neste trabalho uma aplicação numérica da formulação de pequenas deformações descrita, discute-se acerca dos algoritmos de retorno apenas na subseção 4.2.6, onde esses são contextualizados na formulação de grandes deformações. 


\subsection{Modelo elasto-plástico em grandes deformações}

Nesta seção, apresenta-se o modelo elasto-plástico efetivamente utilizado nas simulações numéricas deste trabalho. Conforme já comentado na introdução do capítulo, tal modelo é baseado nos trabalhos de Svendsen (1998), Dettmer e Reese (2004) e Pascon e Coda (2013a), sendo válido para o regime de grandes deformações. Diversos conceitos presentes nesta seção são aproveitados do modelo de pequenas deformações (seção 4.1), sendo, portanto, retomados aqui de forma direta.

\subsubsection{Cinemática dos corpos sob regime elasto-plástico}

Devido às incoerências já comentadas à respeito da decomposição de Green-Naghdi (subseção 4.1.2), essa é substituída no presente contexto pela decomposição de Kröner-Lee (ou multiplicativa), amplamente utilizada na literatura, e bem aceita no contexto de grandes deformações.

\subsubsection{Decomposição de Kröner-Lee}

De maneira análoga à seção 2.1, considere um corpo em suas configurações inicial e atual, denotadas respectivamente por $\Omega^{0}$ e $\Omega^{1}$. Em Kröner (1960) e Lee (1969), introduz-se o conceito de configuração intermediária: suponha que o corpo é descarregado de forma que chegue a um estado de tensões nula, denotado por $\Omega^{p}$. Nessa situação, as únicas deformações presentes no sólido serão as plásticas. Portanto, considera-se que as deformações elásticas são aquelas que ocorrem ao longo da passagem da configuração $\Omega^{p}$ para $\Omega^{1}$.

Seja $\boldsymbol{\zeta}^{p}$ a função que mapeia os pontos da configuração $\Omega^{0} \mathrm{em} \Omega^{p}$, e $\boldsymbol{\zeta}^{e}$ a função que mapeia mapeia os pontos da configuração $\Omega^{p}$ em $\Omega^{1}$ (como ilustra a Figura 15), e sejam os gradientes:

$$
\begin{gathered}
\mathbf{A}^{e}=\nabla_{\zeta^{p}(\mathbf{x})}\left(\boldsymbol{\zeta}^{e}\right), \quad \mathrm{e} \\
\mathbf{A}^{p}=\nabla_{\mathbf{x}}\left(\boldsymbol{\zeta}^{p}\right),
\end{gathered}
$$

onde $\boldsymbol{\nabla}_{\zeta^{p}(\mathbf{x})}$ denota o gradiente com relação à configuração intermediária. Então o gradiente da função mudança de configuração $\boldsymbol{\zeta}$ pode ser escrito como:

$$
\mathbf{A}=\nabla_{\mathbf{x}}(\boldsymbol{\zeta})=\nabla_{\mathbf{x}}\left(\boldsymbol{\zeta}^{e} \circ \boldsymbol{\zeta}^{p}\right)=\mathbf{A}^{e} \mathbf{A}^{p} .
$$

A equação (4.32) é conhecida como decomposição de Kröner-Lee. Vale ser ressaltado que, da maneira que foi definida, essa decomposição não é única, pois pode ser gerada por qualquer configuração intermediária que resulta de um movimento de corpo rígido em $\Omega^{p}$. Para contornar tal problema, assume-se que a passagem de $\Omega^{p}$ para $\Omega^{1}$ envolve apenas deformações puras, isto é, o tensor rotação da decomposição polar de $\mathbf{A}^{e}$ é a identidade (KHAN; HUANG, 1995).

Adicionalmente, a fim de representar os efeitos do encruamento cinemático, utiliza-se 
uma segunda decomposição multiplicativa:

$$
\mathbf{A}^{p}=\mathbf{A}_{e}^{p} \mathbf{A}_{i}^{p},
$$

onde $\mathbf{A}_{e}^{p}$ representa as parcelas plásticas elásticas de $\mathbf{A}^{p}$, e $\mathbf{A}_{i}^{p}$ representa as parcelas plásticas irreversíveis, de forma análoga ao apresentado no caso de pequenas deformações. As duas decomposições descritas podem ser visualizadas de forma esquemática na Figura 15.

Figura 15 - Esquema de configurações intermediárias do modelo elasto-plástico

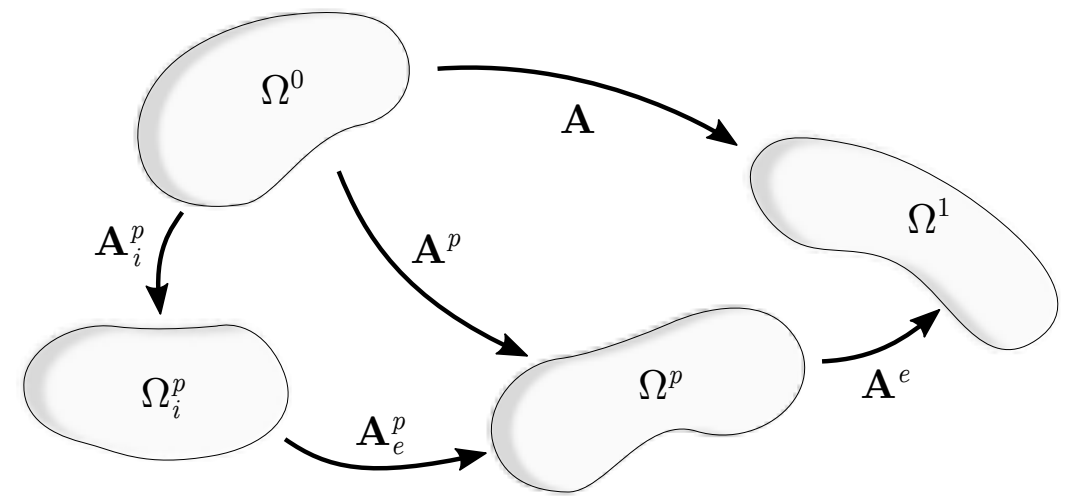

\subsubsection{Deformações elásticas e plásticas}

Partindo da Equação (2.5), podemos definir as parcelas elásticas e plásticas do tensor de deformações de Green-Lagrange, respectivamente, como:

$$
\begin{aligned}
& \mathbf{E}^{e}=\frac{1}{2}\left(\left(\mathbf{A}^{e}\right)^{T} \mathbf{A}^{e}-\mathbf{I}\right)=\frac{1}{2}\left(\mathbf{C}^{e}-\mathbf{I}\right), \quad \mathrm{e} \\
& \mathbf{E}^{p}=\frac{1}{2}\left(\left(\mathbf{A}^{p}\right)^{T} \mathbf{A}^{p}-\mathbf{I}\right)=\frac{1}{2}\left(\mathbf{C}^{p}-\mathbf{I}\right) .
\end{aligned}
$$

Segue que

$$
\mathbf{C}=\mathbf{A}^{T} \mathbf{A}=\left(\mathbf{A}^{e} \mathbf{A}^{p}\right)^{T}\left(\mathbf{A}^{e} \mathbf{A}^{p}\right)=\left(\mathbf{A}^{p}\right)^{T}\left(\mathbf{A}^{e}\right)^{T} \mathbf{A}^{e} \mathbf{A}^{p}=\left(\mathbf{A}^{p}\right)^{T} \mathbf{C}^{e} \mathbf{A}^{p},
$$

$\mathrm{Ou}$,

$$
\mathbf{C}^{e}=\left(\mathbf{A}^{p}\right)^{-T} \mathbf{C}\left(\mathbf{A}^{p}\right)^{-1}
$$

A partir disso, pode-se provar que a deformação de Green-Lagrange total é expressa como

$$
\mathbf{E}=\left(\mathbf{A}^{p}\right)^{T} \mathbf{E}^{e} \mathbf{A}^{p}+\mathbf{E}^{p},
$$

isto é, em regime de pequenas deformações plásticas, a decomposição de Kröner-Lee se associa à de Green-Naghdi, uma vez que a parcela $\left(\mathbf{A}^{p}\right)^{T} \mathbf{E}^{e} \mathbf{A}^{p}$ é muito próxima de $\mathbf{E}^{e}$.

Definem-se, ainda, as deformações

$$
\begin{aligned}
& \mathbf{E}_{e}^{p}=\frac{1}{2}\left(\left(\mathbf{A}_{e}^{p}\right)^{T} \mathbf{A}_{e}^{p}-\mathbf{I}\right)=\frac{1}{2}\left(\mathbf{C}_{e}^{p}-\mathbf{I}\right), \quad \mathrm{e} \\
& \mathbf{E}_{i}^{p}=\frac{1}{2}\left(\left(\mathbf{A}_{i}^{p}\right)^{T} \mathbf{A}_{i}^{p}-\mathbf{I}\right)=\frac{1}{2}\left(\mathbf{C}_{i}^{p}-\mathbf{I}\right),
\end{aligned}
$$

de onde resulta, analogamente à (4.37), a relação:

$$
\mathbf{C}_{e}^{p}=\left(\mathbf{A}_{i}^{p}\right)^{-T} \mathbf{C}^{p}\left(\mathbf{A}_{i}^{p}\right)^{-1}
$$




\subsubsection{Taxas de deformação}

Aplicando (4.32) em (2.6), temos:

$$
\begin{aligned}
\mathbf{L} & =\dot{\mathbf{A}} \mathbf{A}^{-1}=\left(\dot{\mathbf{A}} \mathbf{A}^{p}+\mathbf{A}^{e} \dot{\mathbf{A}}^{p}\right)\left(\mathbf{A}^{e} \mathbf{A}^{p}\right)^{-1} \\
& =\dot{\mathbf{A}} \mathbf{A}^{p}\left(\mathbf{A}^{p}\right)^{-1}\left(\mathbf{A}^{e}\right)^{-1}+\mathbf{A}^{e} \dot{\mathbf{A}}^{p}\left(\mathbf{A}^{p}\right)^{-1}\left(\mathbf{A}^{e}\right)^{-1} \\
& =\dot{\mathbf{A}}^{e}\left(\mathbf{A}^{e}\right)^{-1}+\mathbf{A}^{e} \dot{\mathbf{A}}^{p}\left(\mathbf{A}^{p}\right)^{-1}\left(\mathbf{A}^{e}\right)^{-1} \\
& =\mathbf{L}^{e}+\mathbf{A}^{e} \mathbf{L}^{p}\left(\mathbf{A}^{e}\right)^{-1},
\end{aligned}
$$

onde

$$
\mathbf{L}^{e}=\dot{\mathbf{A}}^{e}\left(\mathbf{A}^{e}\right)^{-1} \quad \text { e } \quad \mathbf{L}^{p}=\dot{\mathbf{A}}^{p}\left(\mathbf{A}^{p}\right)^{-1},
$$

que, por sua vez, podem ser decompostos em suas respectivas parcelas simétricas, $\mathbf{D}^{e} \mathrm{e}$ $\mathbf{D}^{p}$, e antissimétricas, $\mathbf{W}^{e}$ e $\mathbf{W}^{p}$. Consequentemente,

$$
\begin{aligned}
& \mathbf{D}=\operatorname{sim}(\mathbf{L})=\operatorname{sim}\left(\mathbf{L}^{e}\right)+\operatorname{sim}\left(\mathbf{A}^{e} \mathbf{L}^{p}\left(\mathbf{A}^{e}\right)^{-1}\right)=\mathbf{D}^{e}+\operatorname{sim}\left(\mathbf{A}^{e} \mathbf{L}^{p}\left(\mathbf{A}^{e}\right)^{-1}\right), \quad \mathrm{e} \\
& \mathbf{W}=\operatorname{ant}(\mathbf{L})=\operatorname{ant}\left(\mathbf{L}^{e}\right)+\operatorname{ant}\left(\mathbf{A}^{e} \mathbf{L}^{p}\left(\mathbf{A}^{e}\right)^{-1}\right)=\mathbf{W}^{e}+\operatorname{ant}\left(\mathbf{A}^{e} \mathbf{L}^{p}\left(\mathbf{A}^{e}\right)^{-1}\right) .
\end{aligned}
$$

Além disso, seguindo os mesmos passos de (2.9), podemos obter as relações

$$
\begin{aligned}
& \mathbf{D}^{e}=\left(\mathbf{A}^{e}\right)^{-T} \dot{\mathbf{E}}^{e}\left(\mathbf{A}^{e}\right)^{-1}, \quad \mathrm{e} \\
& \mathbf{D}^{p}=\left(\mathbf{A}^{p}\right)^{-T} \dot{\mathbf{E}}^{p}\left(\mathbf{A}^{p}\right)^{-1} .
\end{aligned}
$$

Finalmente, aplicando (4.44) em (2.10), resulta

$$
\begin{aligned}
\dot{\mathbf{E}} & =\mathbf{A}^{T} \mathbf{D} \mathbf{A}=\left(\mathbf{A}^{e} \mathbf{A}^{p}\right)^{T}\left(\mathbf{D}^{e}+\operatorname{sim}\left(\mathbf{A}^{e} \mathbf{L}^{p}\left(\mathbf{A}^{e}\right)^{-1}\right)\right)\left(\mathbf{A}^{e} \mathbf{A}^{p}\right) \\
& =\left(\mathbf{A}^{p}\right)^{T}\left(\mathbf{A}^{e}\right)^{T}\left[\mathbf{D}^{e}+\frac{1}{2} \mathbf{A}^{e} \mathbf{L}^{p}\left(\mathbf{A}^{e}\right)^{-1}+\frac{1}{2}\left(\mathbf{A}^{e}\right)^{-T}\left(\mathbf{L}^{p}\right)^{T}\left(\mathbf{A}^{e}\right)^{T}\right] \mathbf{A}^{e} \mathbf{A}^{p} \\
& =\left(\mathbf{A}^{p}\right)^{T}\left(\mathbf{A}^{e}\right)^{T} \mathbf{D}^{e} \mathbf{A}^{e} \mathbf{A}^{p}+\frac{1}{2}\left(\mathbf{A}^{p}\right)^{T} \mathbf{C}^{e} \mathbf{L}^{p} \mathbf{A}^{p}+\frac{1}{2}\left(\mathbf{A}^{p}\right)^{T}\left(\mathbf{L}^{p}\right)^{T} \mathbf{C}^{e} \mathbf{A}^{p} \\
& =\left(\mathbf{A}^{p}\right)^{T} \dot{\mathbf{E}}^{e} \mathbf{A}^{p}+\frac{1}{2}\left(\mathbf{A}^{p}\right)^{T} \mathbf{C}^{e} \mathbf{L}^{p} \mathbf{A}^{p}+\frac{1}{2}\left(\mathbf{A}^{p}\right)^{T}\left(\mathbf{L}^{p}\right)^{T} \mathbf{C}^{e} \mathbf{A}^{p} .
\end{aligned}
$$

ou, rearranjando,

$$
\dot{\mathbf{E}}^{e}=\left(\mathbf{A}^{p}\right)^{-T} \dot{\mathbf{E}}\left(\mathbf{A}^{p}\right)^{-1}-\frac{1}{2} \mathbf{C}^{e} \mathbf{L}^{p}-\frac{1}{2}\left(\mathbf{L}^{p}\right)^{T} \mathbf{C}^{e} .
$$

Analogamente à equação (4.42), temos:

$$
\mathbf{L}^{p}=\dot{\mathbf{A}}^{p}\left(\mathbf{A}^{p}\right)^{-1}=\mathbf{L}_{e}^{p}+\mathbf{A}_{e}^{p} \mathbf{L}_{i}^{p}\left(\mathbf{A}_{e}^{p}\right)^{-1}
$$

onde,

$$
\mathbf{L}_{e}^{p}=\dot{\mathbf{A}}_{e}^{p}\left(\mathbf{A}_{e}^{p}\right)^{-1} \quad \text { e } \quad \mathbf{L}_{i}^{p}=\dot{\mathbf{A}}_{i}^{p}\left(\mathbf{A}_{i}^{p}\right)^{-1} .
$$

Além disso, pode-se definir:

$$
\begin{aligned}
\mathbf{D}_{e}^{p} & =\left(\mathbf{A}_{e}^{p}\right)^{-T} \dot{\mathbf{E}}_{e}^{p}\left(\mathbf{A}_{e}^{p}\right)^{-1}, \quad \mathrm{e} \\
\mathbf{D}_{i}^{p} & =\left(\mathbf{A}_{i}^{p}\right)^{-T} \dot{\mathbf{E}}_{i}^{p}\left(\mathbf{A}_{i}^{p}\right)^{-1},
\end{aligned}
$$

e, analogamente à equação (4.49), resulta a relação:

$$
\dot{\mathbf{E}}_{e}^{p}=\left(\mathbf{A}_{i}^{p}\right)^{-T} \dot{\mathbf{E}}^{p}\left(\mathbf{A}_{i}^{p}\right)^{-1}-\frac{1}{2} \mathbf{C}_{e}^{p} \mathbf{L}_{i}^{p}-\frac{1}{2}\left(\mathbf{L}_{i}^{p}\right)^{T} \mathbf{C}_{e}^{p} .
$$




\subsubsection{Energia, tensão e dissipação}

Conforme apresentado na subseção 4.1.3, escreve-se a energia livre de Helmholtz pela decomposição aditiva:

$$
\Psi\left(\mathbf{E}^{e}, \mathbf{E}_{e}^{p}, \kappa\right)=\Psi^{e}\left(\mathbf{E}^{e}\right)+\Psi_{c i n}^{p}\left(\mathbf{E}_{e}^{p}\right)+\Psi_{i s o}^{p}(\kappa) .
$$

Com base nisso, deriva-se a tensão elástica, tensão de retorno (back stress), e a tensão de escoamento, respectivamente, pelas expressões

$$
\mathbf{S}^{e}=\frac{\partial \Psi^{e}}{\partial \mathbf{E}^{e}}=2 \frac{\partial \Psi^{e}}{\partial \mathbf{C}^{e}}, \quad \mathbf{X}=\frac{\partial \Psi_{c i n}^{p}}{\partial \mathbf{E}_{e}^{p}}=2 \frac{\partial \Psi_{c i n}^{p}}{\partial \mathbf{C}_{e}^{p}} \quad \text { e } \quad \sigma_{\kappa}=\frac{\partial \Psi_{i s o}^{p}}{\partial \kappa},
$$

e definem-se os operadores:

$$
\mathfrak{C}^{e}=\frac{\partial \mathbf{S}^{e}}{\partial \mathbf{E}^{e}}=\frac{\partial^{2} \Psi^{e}}{\partial \mathbf{E}^{e} \otimes \partial \mathbf{E}^{e}} \quad \text { e } \quad \mathfrak{C}_{e}^{p}=\frac{\partial \mathbf{X}}{\partial \mathbf{E}_{e}^{p}}=\frac{\partial^{2} \Psi_{c i n}^{p}}{\partial \mathbf{E}_{e}^{p} \otimes \partial \mathbf{E}_{e}^{p}}
$$

Tomando-se para $\Psi^{e}$ e $\Psi_{c i n}^{p}$ a lei hiperelástica neo-Hookeana apresentada na subseção 2.5.2, temos:

$$
\begin{gathered}
\Psi^{e}=\frac{\Lambda^{e}}{2} \ln \left(J^{e}\right)^{2}+\frac{\mu^{e}}{2}\left[\operatorname{tr}\left(\mathbf{C}^{e}\right)-3-2 \ln \left(J^{e}\right)\right], \quad \mathrm{e} \\
\Psi_{\text {cin }}^{p}=\frac{c}{4}\left[\operatorname{tr}\left(\mathbf{C}^{e}\right)-3-2 \ln \left(J^{e}\right)\right]
\end{gathered}
$$

onde $\Lambda^{e}, \mu^{e}$ e $c$ são parâmetros do material e $J^{e}=\operatorname{det}\left(\mathbf{A}^{e}\right)$ é o jacobiano elástico. A partir de (4.58) e (4.59), pode-se calcular os tensores $\mathbf{S}^{e}$ e $\mathbf{X}$, respectivamente, por uma expressão análoga à (2.80). As leis para $\sigma_{\kappa}$ podem ser obtidas diretamente da Tabela 1 , de onde pode-se integrar expressões para $\Psi_{i s o}^{p}$, caso necessário.

Utilizando-se novamente a desigualdade de Clausius-Duhem (4.13) para representar a segunda lei da termodinâmica, escreve-se:

$$
d_{i n t}=\mathbf{S}: \dot{\mathbf{E}}-\mathbf{S}^{e}: \dot{\mathbf{E}}^{e}-\mathbf{X}: \dot{\mathbf{E}}_{e}^{p}-\sigma_{\kappa} \dot{\kappa} \geq 0 .
$$

Da equação (4.49), pode-se obter:

$$
\mathbf{S}^{e}: \dot{\mathbf{E}}^{e}=\mathbf{S}^{e}:\left(\left(\mathbf{A}^{p}\right)^{-T} \dot{\mathbf{E}}\left(\mathbf{A}^{p}\right)^{-1}-\frac{1}{2} \mathbf{C}^{e} \mathbf{L}^{p}-\frac{1}{2}\left(\mathbf{L}^{p}\right)^{T} \mathbf{C}^{e}\right)
$$

de onde, usando as relações tensoriais (A.33) e (A.34), resulta:

$$
\begin{aligned}
\mathbf{S}^{e}: \dot{\mathbf{E}}^{e} & =\left(\left(\mathbf{A}^{p}\right)^{-1} \mathbf{S}^{e}\left(\mathbf{A}^{p}\right)^{-T}\right): \dot{\mathbf{E}}-\frac{1}{2} \mathbf{C}^{e} \mathbf{S}^{e}: \mathbf{L}^{p}-\frac{1}{2} \mathbf{C}^{e} \mathbf{S}^{e}: \mathbf{L}^{p} \\
& =\left(\left(\mathbf{A}^{p}\right)^{-1} \mathbf{S}^{e}\left(\mathbf{A}^{p}\right)^{-T}\right): \dot{\mathbf{E}}-\mathbf{M}^{e}: \mathbf{L}^{p}
\end{aligned}
$$

onde

$$
\mathbf{M}^{e}=\mathbf{C}^{e} \mathbf{S}^{e}
$$

é denominado tensor de Mandel, definido na configuração intermediária. Analogamente,

$$
\mathbf{X}: \dot{\mathbf{E}}_{e}^{p}=\left(\left(\mathbf{A}_{i}^{p}\right)^{-1} \mathbf{X}\left(\mathbf{A}_{i}^{p}\right)^{-T}\right): \dot{\mathbf{E}}^{p}-\mathbf{M}_{e}^{p}: \mathbf{L}_{i}^{p}
$$

onde $\mathbf{M}_{e}^{p}$ é um tensor do tipo Mandel dado por

$$
\mathbf{M}_{e}^{p}=\mathbf{C}_{e}^{p} \mathbf{X} .
$$


Aplicando ainda as equações (4.47) e (A.33), escreve-se:

$$
\mathbf{X}: \dot{\mathbf{E}}_{e}^{p}=\left(\mathbf{A}^{p}\left(\mathbf{A}_{i}^{p}\right)^{-1} \mathbf{X}\left(\mathbf{A}_{i}^{p}\right)^{-T}\left(\mathbf{A}^{p}\right)^{T}\right): \mathbf{D}^{p}-\mathbf{M}_{e}^{p}: \mathbf{L}_{i}^{p}=\boldsymbol{\chi}: \mathbf{D}^{p}-\mathbf{M}_{e}^{p}: \mathbf{L}_{i}^{p},
$$

onde

$$
\chi=\mathbf{A}_{e}^{p} \mathbf{X}\left(\mathbf{A}_{e}^{p}\right)^{T}
$$

é denominada tensão de retorno na configuração intermediária. Da simetria de $\mathbf{X}$, segue que $\chi$ é simétrico. Dessa forma, pela relação tensorial (A.40),

$$
\chi: \mathbf{D}^{p}=\chi: \operatorname{sim}\left(\mathbf{L}^{p}\right)=\chi: \mathbf{L}^{p}
$$

$\log \mathrm{O}$

$$
\mathbf{X}: \dot{\mathbf{E}}_{e}^{p}=\chi: \mathbf{L}^{p}-\mathbf{M}_{e}^{p}: \mathbf{L}_{i}^{p}
$$

Finalmente, aplicando (4.62) e (4.69) em (4.60), resulta

$$
d_{i n t}=\left(\mathbf{S}-\left(\mathbf{A}^{p}\right)^{-1} \mathbf{S}^{e}\left(\mathbf{A}^{p}\right)^{-T}\right): \dot{\mathbf{E}}+\left(\mathbf{M}^{e}-\boldsymbol{\chi}\right): \mathbf{L}^{p}+\mathbf{M}_{e}^{p}: \mathbf{L}_{i}^{p}-\sigma_{\kappa} \dot{\kappa} .
$$

Para garantir a não-negatividade da energia de dissipação para valores arbitrários de $\dot{\mathbf{E}}$ (HOLZAPFEL, 2000), define-se a tensão de Piola-Kirchhoff de segunda espécie como

$$
\mathbf{S}=\left(\mathbf{A}^{p}\right)^{-1} \mathbf{S}^{e}\left(\mathbf{A}^{p}\right)^{-T},
$$

logo, a energia de dissipação é reduzida para:

$$
d_{i n t}=\left(\mathbf{M}^{e}-\boldsymbol{\chi}\right): \mathbf{L}^{p}+\mathbf{M}_{e}^{p}: \mathbf{L}_{i}^{p}-\sigma_{\kappa} \dot{\kappa} \geq 0 .
$$

É interessante observar que, neste modelo, o tensor $\mathbf{M}^{e}-\boldsymbol{\chi}$ é termodinamicamente conjugado a $\mathbf{L}^{p}$ (SVENDSEN, 1998) e, portanto, é utilizado para definir o critério de plastificação. Caso $\Psi^{e}$ seja isotrópico, temos ainda que $\mathbf{M}^{e}$ é simétrico (SVENDSEN et al., 1998), logo, pela relação tensorial (A.40),

$$
\left(\mathbf{M}^{e}-\chi\right): \mathbf{L}^{p}=\left(\mathbf{M}^{e}-\chi\right): \operatorname{sim}\left(\mathbf{L}^{p}\right)=\left(\mathbf{M}^{e}-\chi\right): \mathbf{D}^{p}
$$

e, portanto, $\mathbf{M}^{e}-\chi$ é termodinamicamente conjugado à taxa de deformação plástica.

\subsubsection{Critério de plastificação}

Adaptando o critério de von Mises apresentado na subseção 4.1.4 para o presente contexto, escreve-se

$$
\Phi\left(\mathbf{M}^{e}, \boldsymbol{\chi}, \sigma_{\kappa}\right)=\left\|\left(\mathbf{M}^{e}-\chi\right)^{D}\right\|-\sqrt{\frac{2}{3}} \sigma_{\kappa} \leq 0 .
$$

É possível mostrar que, quando as deformações tendem a zero, o tensor $\mathbf{M}^{e}-\chi$ aproxima-se de $\mathbf{S}^{e}-\mathbf{X}$, restaurando o critério da equação (4.17). Além disso,

$$
\begin{aligned}
\mathbf{M}^{e} & =\mathbf{C}^{e} \mathbf{S}^{e}=\left(\mathbf{A}^{e}\right)^{T} \mathbf{A}^{e} \mathbf{A}^{p} \mathbf{S}\left(\mathbf{A}^{p}\right)^{T} \\
& =\left(\mathbf{A}^{e}\right)^{T} \mathbf{A}^{e} \mathbf{A}^{p} \mathbf{S}\left(\mathbf{A}^{p}\right)^{T}\left(\mathbf{A}^{e}\right)^{T}\left(\mathbf{A}^{e}\right)^{-T} \\
& =\left(\mathbf{A}^{e}\right)^{T} \mathbf{A} \mathbf{S} \mathbf{A}^{T}\left(\mathbf{A}^{e}\right)^{-T} \\
& =\left(\mathbf{A}^{e}\right)^{T} \boldsymbol{\tau}\left(\mathbf{A}^{e}\right)^{-T}
\end{aligned}
$$


onde

$$
\boldsymbol{\tau}=\mathbf{A S A}^{T}=J \boldsymbol{\sigma}
$$

é denominada tensão de Kirchhoff. Da relação tensorial (A.37), temos, ainda,

$$
\left(\mathbf{M}^{e}\right)^{D}=\left(\mathbf{A}^{e}\right)^{T} \boldsymbol{\tau}^{D}\left(\mathbf{A}^{e}\right)^{-T}
$$

Sabendo-se que ambos $\boldsymbol{\tau}$ e $\mathbf{M}^{e}$ são simétricos, a relação (A.36) implica que

$$
\left(\mathbf{M}^{e}\right)^{D}:\left(\mathbf{M}^{e}\right)^{D}=\boldsymbol{\tau}^{D}: \boldsymbol{\tau}^{D} \quad \text { ou } \quad\left\|\left(\mathbf{M}^{e}\right)^{D}\right\|=\left\|\boldsymbol{\tau}^{D}\right\|=J\left\|\boldsymbol{\sigma}^{D}\right\|,
$$

mostrando que o tensor de Mandel, apesar de ter uma definição a princípio abstrata, associa-se com um tensor de tensões que possui um significado físico e pode ser medido em laboratório. Isso facilita ainda a associação do critério adotado com outros que utilizam o tensor de Kirchhoff, comumente adotados em formulações elasto-plásticas de grandes deformações, uma vez que, quando $\boldsymbol{\chi}=\mathbf{0}$, temos $\Phi\left(\mathbf{M}^{e}, \boldsymbol{\chi}, \sigma_{\kappa}\right)=\Phi\left(\boldsymbol{\tau}, \boldsymbol{\chi}, \sigma_{\kappa}\right)$.

\subsubsection{Leis de evolução}

Assim como no caso de pequenas deformações (subseção 4.1.6), pode-se aplicar o princípio da máxima dissipação da energia, que, nesse caso, consiste em minimizar o funcional

$$
\ell\left(\mathbf{M}^{e}-\chi, \mathbf{M}_{e}^{p}, \sigma_{\kappa}, \dot{\gamma}\right)=-\left(\mathbf{M}^{e}-\chi\right): \mathbf{L}^{p}-\mathbf{M}_{e}^{p}: \mathbf{L}_{i}^{p}+\sigma_{\kappa} \dot{\kappa}+\dot{\gamma} \Phi
$$

onde para ignorar a restrição $\Phi=0$ nas etapas puramente elásticas, utiliza-se novamente as condições de complementaridade e consistência, discutidas na subseção 4.1.7. Minimizando $\ell$ com relação aos tensores $\mathbf{M}^{e}-\chi, \mathbf{M}_{e}^{p}$ e $\sigma_{\kappa}$, tem-se:

$$
\left\{\begin{array}{l}
\frac{\partial \ell}{\partial\left(\mathbf{M}^{e}-\boldsymbol{\chi}\right)}=-\mathbf{L}^{p}+\dot{\gamma} \frac{\partial \Phi}{\partial\left(\mathbf{M}^{e}-\boldsymbol{\chi}\right)}=\mathbf{0} \\
\frac{\partial \ell}{\partial \sigma_{\kappa}}=\dot{\kappa}+\dot{\gamma} \frac{\partial \Phi}{\partial \sigma_{\kappa}}=0 \\
\frac{\partial \ell}{\partial \mathbf{M}_{e}^{p}}=\mathbf{L}_{i}^{p}+\dot{\gamma} \frac{\partial \Phi}{\partial \mathbf{M}_{e}^{p}}=\mathbf{0} .
\end{array}\right.
$$

Extrapolando (4.80) para o caso não-associativo, pode-se escrever as leis de evolução

$$
\begin{cases}\mathbf{L}^{p}=\dot{\gamma} \mathbf{N}^{p}, & \text { onde } \mathbf{N}^{p}=\frac{\partial \Omega}{\partial\left(\mathbf{M}^{e}-\boldsymbol{\chi}\right)} \\ \mathbf{L}_{i}^{p}=\dot{\gamma} \mathbf{N}_{i}^{p}, & \text { onde } \mathbf{N}_{i}^{p}=\frac{\partial \Omega}{\partial \mathbf{M}_{e}^{p}} \\ \dot{\kappa}=\dot{\gamma} n_{\kappa}, & \text { onde } n_{\kappa}=-\frac{\partial \Omega}{\partial \sigma_{\kappa}} .\end{cases}
$$

A lei de potencial plástico $\Omega$ adotada nesse caso, é dada por

$$
\Omega=\Phi+\frac{b}{2 c}\left(\mathbf{M}_{e}^{p}\right)^{D}:\left(\mathbf{M}_{e}^{p}\right)^{D}
$$

$\log \mathrm{O}$ :

$$
\mathbf{N}^{p}=\frac{\left(\mathbf{M}^{e}-\chi\right)^{D}}{\left\|\left(\mathbf{M}^{e}-\chi\right)^{D}\right\|}
$$




$$
\begin{gathered}
\mathbf{N}_{i}^{p}=\frac{b}{c}\left(\mathbf{M}_{e}^{p}\right)^{D}, \quad \mathrm{e} \\
n_{\kappa}=\sqrt{\frac{2}{3}} .
\end{gathered}
$$

Aplicando esses resultados, juntamente com as Equações (4.43) e (4.51), em (4.81), podemos finalmente expressar as leis de evolução na forma:

$$
\left\{\begin{array}{l}
\dot{\mathbf{A}}^{p}=\dot{\gamma} \mathbf{N}^{p} \mathbf{A}^{p}, \\
\dot{\mathbf{A}}_{i}^{p}=\dot{\gamma} \mathbf{N}_{i}^{p} \mathbf{A}_{i}^{p}, \\
\dot{\kappa}=\dot{\gamma} \sqrt{\frac{2}{3}} .
\end{array}\right.
$$

A Equação (4.87), apresentada em Vladimirov, Pietryga e Reese (2010), trata-se de uma extensão da lei de Armstrong-Frederick para o regime de grandes deformações.

Deve-se mostrar que as leis de evolução adotadas satisfazem a inequação de ClausiusDuhem. Utilizando a relação tensorial (A.39), escreve-se:

$$
\mathbf{M}_{e}^{p}: \mathbf{L}_{i}^{p}=\dot{\gamma} \frac{b}{c} \mathbf{M}_{e}^{p}:\left(\mathbf{M}_{e}^{p}\right)^{D}=\dot{\gamma} \frac{b}{c}\left(\mathbf{M}_{e}^{p}\right)^{D}:\left(\mathbf{M}_{e}^{p}\right)^{D}=\dot{\gamma} \frac{b}{c}\left\|\left(\mathbf{M}_{e}^{p}\right)^{D}\right\|^{2} .
$$

Analogamente, para as lei de evolução de $\mathbf{L}^{p}$ e $\dot{\kappa}$ têm-se:

$$
\begin{gathered}
\left(\mathbf{M}^{e}-\boldsymbol{\chi}\right): \mathbf{L}^{p}=\dot{\gamma}\left(\mathbf{M}^{e}-\boldsymbol{\chi}\right): \frac{\left(\mathbf{M}^{e}-\boldsymbol{\chi}\right)^{D}}{\left\|\left(\mathbf{M}^{e}-\boldsymbol{\chi}\right)^{D}\right\|}=\dot{\gamma}\left\|\left(\mathbf{M}^{e}-\boldsymbol{\chi}\right)^{D}\right\|, \quad \mathrm{e} \\
\sigma_{\kappa} \dot{\kappa}=\dot{\gamma} \sqrt{\frac{2}{3}} \sigma_{\kappa} .
\end{gathered}
$$

Aplicando as equações (4.89), (4.90) e (4.91) em (4.72), resulta a seguinte expressão para a energia de dissipação:

$$
d_{\text {int }}=\dot{\gamma} \Phi+\dot{\gamma} \frac{b}{c}\left\|\left(\mathbf{M}_{e}^{p}\right)^{D}\right\|^{2}
$$

A primeira parcela de (4.92) se anula pela condição de complementaridade, e a segunda parcela é maior ou igual a zero, uma vez que $\dot{\gamma}$ deve ser maior ou igual a zero e os parâmetros $b$ e $c$ são positivos. Dessa forma, está provado que $d_{i n t} \geq 0$.

Deve-se salientar que, no caso isotrópico, os tensores $\mathbf{N}^{p}$ e $\mathbf{N}_{i}^{p}$ são simétricos. Logo, pelas leis de evolução dadas na Equação (4.81), $\mathbf{L}^{p}$ e $\mathbf{L}_{i}^{p}$ também se tornam simétricos. Isso implica, neste caso, que as vorticidades plásticas são desprezadas, ou seja,

$$
\mathbf{W}^{p}=\mathbf{W}_{i}^{p}=\mathbf{0} .
$$

Tal consideração foi feita também nos trabalhos de Pascon e Coda (2013a) e Eterovic e Bathe (1991).

Resta verificar que a lei de evolução adotada garante a conservação do volume plástico, uma hipótese clássica na teoria da plasticidade. De acordo com essa propriedade, as deformações plásticas não devem provocar mudanças de volume, isto é, o jacobiano plástico $J^{p}=\operatorname{det}\left(\mathbf{A}^{p}\right)$ é constante e igual a 1 . De fato, utilizando a lei de evolução (4.86) 
e as relações tensoriais (A.23) e (A.35), tem-se a taxa do Jacobiano plástico dada por:

$$
\dot{J}^{p}=\frac{d \operatorname{det}\left(\mathbf{A}^{p}\right)}{d \mathbf{A}^{p}}: \dot{\mathbf{A}}^{p}=\dot{\gamma} \operatorname{det}\left(\mathbf{A}^{p}\right)\left(\mathbf{A}^{p}\right)^{-T}: \mathbf{N}^{p} \mathbf{A}^{p}=\dot{\gamma} J^{p} \operatorname{tr}\left(\mathbf{N}^{p}\right),
$$

mas, conforme visto na equação (4.83), $\mathbf{N}^{p}$ é um tensor desviador, e, portanto, $\operatorname{tr}\left(\mathbf{N}^{p}\right)=0$, provando, como desejado, que $\dot{J}^{p}=0$.

\subsubsection{Operador tangente consistente elasto-plástico}

O operador tangente consistente elasto-plástico, definido a partir de (4.71) como

$$
\mathfrak{C}^{e p}=\frac{d \mathbf{S}}{d \mathbf{E}}=\frac{d\left(\mathbf{A}^{p}\right)^{-1}}{d \mathbf{E}} \mathbf{S}^{e}\left(\mathbf{A}^{p}\right)^{-T}+\left(\mathbf{A}^{p}\right)^{-1} \frac{d \mathbf{S}^{e}}{d \mathbf{E}}\left(\mathbf{A}^{p}\right)^{-T}+\left(\mathbf{A}^{p}\right)^{-1} \mathbf{S}^{e} \frac{d\left(\mathbf{A}^{p}\right)^{-T}}{d \mathbf{E}},
$$

possui particular importância na solução numérica do problema, pois sua aplicação na equação (3.37) garante a convergência quadrática do método de Newton-Raphson mesmo em regime elasto-plástico.

Para que seja possível calcular as derivadas $d\left(\mathbf{A}^{p}\right)^{-1} / d \mathbf{E}$ e $d \mathbf{S}^{e} / d \mathbf{E}$, é preciso conhecer o valor de $d \mathbf{A}^{p} / d \mathbf{E}$. Neste trabalho, utiliza-se o procedimento descrito por Pascon e Coda (2013a), que parte da condição de consistência (4.29). Assim tem-se:

$$
\begin{aligned}
\dot{\Phi} & =\frac{\partial \Phi}{\partial \mathbf{E}}: \dot{\mathbf{E}}+\frac{\partial \Phi}{\partial \mathbf{A}^{p}}: \dot{\mathbf{A}^{p}}+\frac{\partial \Phi}{\partial \kappa}: \dot{\kappa}+\frac{\partial \Phi}{\partial \mathbf{A}_{i}^{p}}: \dot{\mathbf{A}}_{i}^{p} \\
& =\frac{\partial \Phi}{\partial \mathbf{E}}: \dot{\mathbf{E}}+\dot{\gamma}\left(\frac{\partial \Phi}{\partial \mathbf{A}^{p}}:\left(\mathbf{N}^{p} \mathbf{A}^{p}\right)+\frac{\partial \Phi}{\partial \kappa}: r_{\kappa}+\frac{\partial \Phi}{\partial \mathbf{A}_{i}^{p}}:\left(\mathbf{N}_{i}^{p} \mathbf{A}_{i}^{p}\right)\right)=0
\end{aligned}
$$

que, rearranjando, escreve-se como

$$
\dot{\gamma}=\mathbf{D}^{\gamma}: \dot{\mathbf{E}}
$$

onde

$$
\mathbf{D}^{\gamma}=-\frac{\partial \Phi / \partial \mathbf{E}}{\frac{\partial \Phi}{\partial \mathbf{A}^{p}}:\left(\mathbf{N}^{p} \mathbf{A}^{p}\right)+\frac{\partial \Phi}{\partial \mathbf{A}_{i}^{p}}:\left(\mathbf{N}_{i}^{p} \mathbf{A}_{i}^{p}\right)+\frac{\partial \Phi}{\partial \kappa} r_{\kappa}} .
$$

Aplicando (4.97) em (4.86), e utilizando a relação tensorial (A.31), temos

$$
\dot{\mathbf{A}^{p}}=\dot{\gamma} \mathbf{N}^{p} \mathbf{A}^{p}=\left(\mathbf{D}^{\gamma}: \dot{\mathbf{E}}\right) \mathbf{N}^{p} \mathbf{A}^{p}=\left[\left(\mathbf{N}^{p} \mathbf{A}^{p}\right) \otimes \mathbf{D}^{\gamma}\right]: \dot{\mathbf{E}},
$$

de onde resulta

$$
\frac{d \mathbf{A}^{p}}{d \mathbf{E}}=\left(\mathbf{N}^{p} \mathbf{A}^{p}\right) \otimes \mathbf{D}^{\gamma}
$$

O cálculo completo de $\mathfrak{C}^{e p}$ e $\mathbf{D}^{\gamma}$ é descrito com detalhes no Apêndice C.

\subsubsection{Implementação computacional}

Como já discutido na subseção 4.1.7, a implementação computacional do modelo descrito baseia-se em etapas de previsão e correção em cada passo de tempo analisado. No caso de problemas estáticos, particiona-se a análise em "pseudo" passos de tempo ou passos de carregamento.

Na etapa de previsão, assume-se que o regime é puramente elástico, isto é, considera- 
se que as variáveis plásticas mantêm os valores do passo anterior. Partindo de tal hipótese, calculam-se as tensões e, consequentemente, o valor de $\Phi$. Se $\Phi<0$, o critério de plastificação é atendido, implicando que o ponto analisado realmente encontra-se em regime elástico e, portanto, as tensões calculadas são válidas. Em contrapartida, se $\Phi>0$, deve-se partir para a etapa de correção, ou retorno, onde atualizam-se as variáveis plásticas. Uma vez que a evolução das variáveis plásticas é descrita em forma de taxa (subseção 4.1.6), adota-se um procedimento incremental, no qual as equações (4.88), (4.87) e (4.86) são aproximadas por

$$
\begin{gathered}
\Delta \mathbf{A}^{p}=\mathbf{A}_{s+1}^{p}-\mathbf{A}_{s}^{p}=\Delta \gamma_{s+1} \mathbf{N}^{p} \mathbf{A}^{p}, \\
\Delta \kappa=\kappa_{s+1}-\kappa_{s}=\Delta \gamma_{s+1} \sqrt{2 / 3} \mathrm{e} \\
\Delta \mathbf{A}_{i}^{p}=\left(\mathbf{A}_{i}^{p}\right)_{s+1}-\left(\mathbf{A}_{i}^{p}\right)_{s}=\Delta \gamma_{s+1} \mathbf{N}_{i}^{p} \mathbf{A}_{i}^{p},
\end{gathered}
$$

onde os índices $(\cdot)_{s+1}$ e $(\cdot)_{s}$ representam, respectivamente, os valores das variáveis nos passos atual e anterior (passo de tempo, no caso dinâmico, ou "pseudo" passo de tempo, no caso estático). Nas três equações acima, quatro variáveis são desconhecidas a princípio: $\mathbf{A}_{s+1}^{p}, \kappa_{s+1},\left(\mathbf{A}_{i}^{p}\right)_{s+1}$ e $\Delta \gamma_{s+1}$. Pela condição de Kuhn-Tucker (4.28), impõe-se ainda que

$$
\Phi_{s+1}=0
$$

tornando o sistema determinado.

Nas equações (4.101), (4.102) e (4.103), resta ainda definir em qual passo de tempo as variáveis $\mathbf{N}^{p} \mathbf{A}^{p}$ e $\mathbf{N}_{i}^{p} \mathbf{A}_{i}^{p}$ são calculadas. Se forem considerados os valores do passo anterior, o algoritmo denomina-se explícito. Nesse caso, o sistema é resolvido sem a necessidade de técnicas iterativas, porém a solução torna-se condicionalmente estável, isto é, sua convergência depende do número de passos adotado. No presente trabalho, será utilizado o algoritmo implícito de Euler (backward Euler), onde os valores são considerados no passo atual. Esse procedimento é incondicionalmente estável porém resulta em um sistema não-linear, escrito como:

$$
\left\{\begin{array}{l}
\mathbf{R}_{1}=\mathbf{A}_{s+1}^{p}-\mathbf{A}_{s}^{p}-\Delta \gamma_{s+1} \mathbf{N}_{s+1}^{p} \mathbf{A}_{s+1}^{p}=\mathbf{0} \\
r_{2}=\kappa_{s+1}-\kappa_{s}-\Delta \gamma_{s+1} \sqrt{2 / 3}=0 \\
\mathbf{R}_{3}=\left(\mathbf{A}_{i}^{p}\right)_{s+1}-\left(\mathbf{A}_{i}^{p}\right)_{s}-\Delta \gamma_{s+1}\left(\mathbf{N}_{i}^{p}\right)_{s+1}\left(\mathbf{A}_{i}^{p}\right)_{s+1}=\mathbf{0} \\
r_{4}=\Phi_{s+1}=0 .
\end{array}\right.
$$

A resolução do sistema (4.105) é feita nesse trabalho pelo método de NewtonRaphson, cujo detalhamento pode ser visto no Apêndice B. Na Figura 16 apresenta-se o passo-a-passo do algoritmo de previsão e correção do modelo descrito nesta seção.

A Figura 17 ilustra a diferença entre os algoritmos explícitos e o algoritmo implícito de Euler no caso de pequenas deformações. No algoritmo implícito, a tensão tentativa $\mathbf{S}_{s+1}^{t e n}$ retorna à superfície de plastificação seguindo a direção $\mathbf{N}_{s+1}^{p}$, isto é, pela direção radial. Já no algoritmo explícito, a tensão é corrigida seguindo a direção $\mathbf{N}_{s}^{p}$, o que pode causar, quando utilizados passos de tempo muito grandes, situações como a da Figura 17a. 
Figura 16 - Algoritmo de previsão e correção do modelo elasto-plástico

\section{ALGORITMO DE PREVISÃO E CORREÇÃO \\ PARA O PASSO DE TEMPO $s+1$}

\section{PREVISÃO ELÁSTICA:}

a) Assume-se como tentativa inicial os valores do passo anterior, isto é,

$$
\mathbf{A}_{s+1}^{p}=\mathbf{A}_{s}^{p}, \quad \kappa_{s+1}=\kappa_{s} \quad \text { e } \quad\left(\mathbf{A}_{i}^{p}\right)_{s+1}=\left(\mathbf{A}_{i}^{p}\right)_{s},
$$

ou, caso seja o primeiro passo $(s=0)$ :

$$
\mathbf{A}_{s+1}^{p}=\mathbf{I}, \quad \kappa_{s+1}=0 \quad \text { e } \quad\left(\mathbf{A}_{i}^{p}\right)_{s+1}=\mathbf{I} .
$$

b) De $\kappa_{s+1}$, calcula-se $\sigma_{\kappa}$ conforme a Tabela 1. De $\mathbf{A}_{s+1}^{p}$ e $\left(\mathbf{A}_{i}^{p}\right)_{s+1}$, calcula-se

$$
\begin{aligned}
\mathbf{A}^{e} & =\mathbf{A}\left(\mathbf{A}^{p}\right)^{-1} \\
\mathbf{C}^{e} & =\left(\mathbf{A}^{e}\right)^{T} \mathbf{A}^{e} \\
\mathbf{S}^{e} & =2 \frac{\partial \Psi^{e}}{\partial \mathbf{C}^{e}} \\
\mathbf{M}^{e} & =\mathbf{C}^{e} \mathbf{S}^{e}
\end{aligned}
$$

$$
\begin{aligned}
\mathbf{A}_{e}^{p} & =\mathbf{A}^{p}\left(\mathbf{A}_{i}^{p}\right)^{-1} \\
\mathbf{C}_{e}^{p} & =\left(\mathbf{A}_{e}^{p}\right)^{T} \mathbf{A}_{e}^{p} \\
\mathbf{X} & =2 \frac{\partial \Psi_{c i n}^{p}}{\partial \mathbf{C}_{e}^{p}} \\
\boldsymbol{\chi} & =\left(\mathbf{A}_{e}^{p}\right) \mathbf{X}\left(\mathbf{A}_{e}^{p}\right)^{T}
\end{aligned}
$$

onde os índices $(\cdot)_{s+1}$ foram omitidos a fim de reduzir a notação.

c) Calcula-se o critério de plastificação

$$
\Phi=\left\|\left(\mathbf{M}^{e}-\chi\right)^{D}\right\|-\sqrt{2 / 3} \sigma_{\kappa} .
$$

2. SE $\Phi \leq 0 \rightarrow$ PREVISÃO ELÁSTICA CORRETA:

a) Não há necessidade de atualizar as variáveis previamente calculadas.

b) Calcula-se $\mathfrak{C}^{e p}$ pela forma simplificada exibida na subseção C.1.1.

3. $\mathrm{SE} \Phi>0 \rightarrow$ CORREÇÃO PLÁSTICA:

a) Soluciona-se o sistema (4.105) conforme o Apêndice B, de onde obtêm-se as variáveis atualizadas $\mathbf{A}_{s+1}^{p}, \kappa_{s+1},\left(\mathbf{A}_{i}^{p}\right)_{s+1}$ e $\Delta \gamma_{s+1}$. Todas essas variáveis, com exceção de $\Delta \gamma_{s+1}$, devem ser armazenadas para serem utilizadas no próximo passo.

b) Atualiza-se $\mathbf{S}_{s+1}^{e}$ e as demais variáveis que serão utilizadas para calcular $\mathfrak{C}^{e p}$.

c) Calcula-se $\mathfrak{C}^{e p}$ conforme a subseção C.1.2.

4. Finalmente, calcula-se o tensor de tensões de Piola-Kirchhoff de segunda espécie:

$$
\mathbf{S}_{s+1}=\left(\mathbf{A}^{p}\right)_{s+1}^{-1} \mathbf{S}_{s+1}^{e}\left(\mathbf{A}^{p}\right)_{s+1}^{-T}
$$

Conforme destacado por Dettmer e Reese (2004), Vladimirov, Pietryga e Reese (2007), o método implícito de Euler pode resultar em pequenos erros quanto à propriedade da conservação do volume plástico (subseção 4.2.4), e, portanto, requer um número de 
Figura 17 - Representação do retorno para algoritmos explícitos e implícitos

(a) Algoritmo explícito

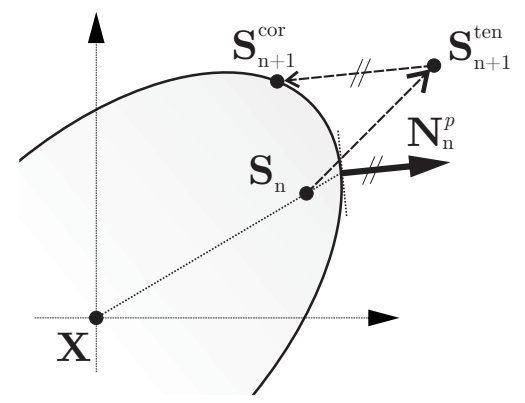

(b) Algoritmo implícito

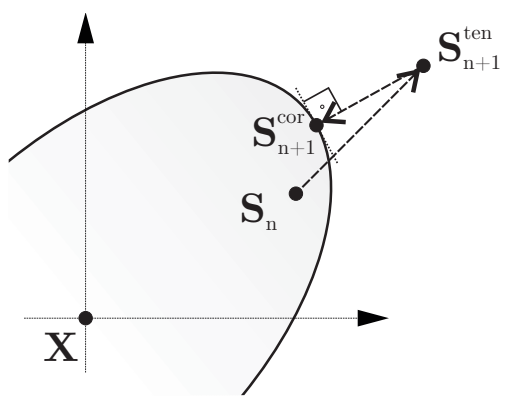

passos suficientemente grande para evitar discrepâncias, como pode ser visto em Tsakmakis e Willuweit (2003). Em Dettmer e Reese (2004), realiza-se um procedimento de integração temporal baseado em funções exponenciais de forma a contornar tal problema.

A respeito da implementação em 2D, utilizam-se os estados planos de deformações e de tensões, conforme apresentado na subseção 2.5.3. Destaca-se que, mesmo no EPD, deve-se utilizar tensores completos $3 \times 3$ para representar as grandezas envolvidas, uma vez que, devido ao caráter desviador das leis de evolução, a condição $E_{33}=0$ não implica em $E_{33}^{p}=0$ ou $E_{33}^{e}=0$ (BONET; WOOD, 1997). Com relação à utilização de estado plano de tensões em problemas de elasto-plasticidade em grandes deformações, o trabalho de Kirchner, Reese e Wriggers (1997) pode ser citado.

\subsubsection{Exemplos de verificação}

A seguir são apresentados dois exemplos numéricos clássicos para verificação do modelo elasto-plástico adotado, propostos originalmente em Dettmer e Reese (2004). No primeiro exemplo, o sólido é submetido apenas a tensões normais de tração e compressão, enquanto no segundo o sólido apresenta deformações e tensões puramente cisalhantes.

Para cada caso, dois materiais são considerados: um metal (Mild Steel Ck15), cujos parâmetros são dados por Lührs, Hartmann e Haupt (1997), e um polímero (PET orientado), cujos parâmetros são dados por Dettmer e Reese (2004). Em ambos, considerase que não há encruamento isotrópico, isto é, a função de escoamento $\sigma_{\kappa}$ é igual a um valor fixo $\sigma_{Y}$, e o encruamento cinemático segue a lei de Armstrong-Frederick. Para a parcela elástica das deformações, adota-se a lei neo-Hookeana dada na equação (4.58). Todos os parâmetros são apresentados na Tabela 2.

Tabela 2 - Parâmetros dos materiais utilizados nos exemplos de validação

\begin{tabular}{cccccc}
\hline Material & $\Lambda^{e}(\mathrm{MPa})$ & $\mu^{e}(\mathrm{MPa})$ & $\sigma_{Y}(\mathrm{MPa})$ & $c(\mathrm{MPa})$ & $b$ \\
\hline Metálico & 173333 & 80000 & 300 & 1900 & 8,5 \\
Polimérico & 320 & 80 & 35 & 100 & 2,7 \\
\hline
\end{tabular}




\subsubsection{Chapa sob tensão uniaxial}

O primeiro exemplo tem como intuito fornecer o diagrama tensão-deformação de um sólido sujeito a tensão uniaxial. Para tal, considera-se uma chapa restrita horizontalmente na face esquerda e verticalmente na face inferior. Aplica-se deslocamento horizontal prescrito na face direita conforme gráfico da Figura 18, com intensidade máxima de $u_{1}=0,5 \mathrm{~m}$ para o material metálico e $u_{1}=1,5 \mathrm{~m}$ para o material polimérico. Foram considerados 1200 passos no total.

Figura 18 - Chapa sob tensão uniaxial

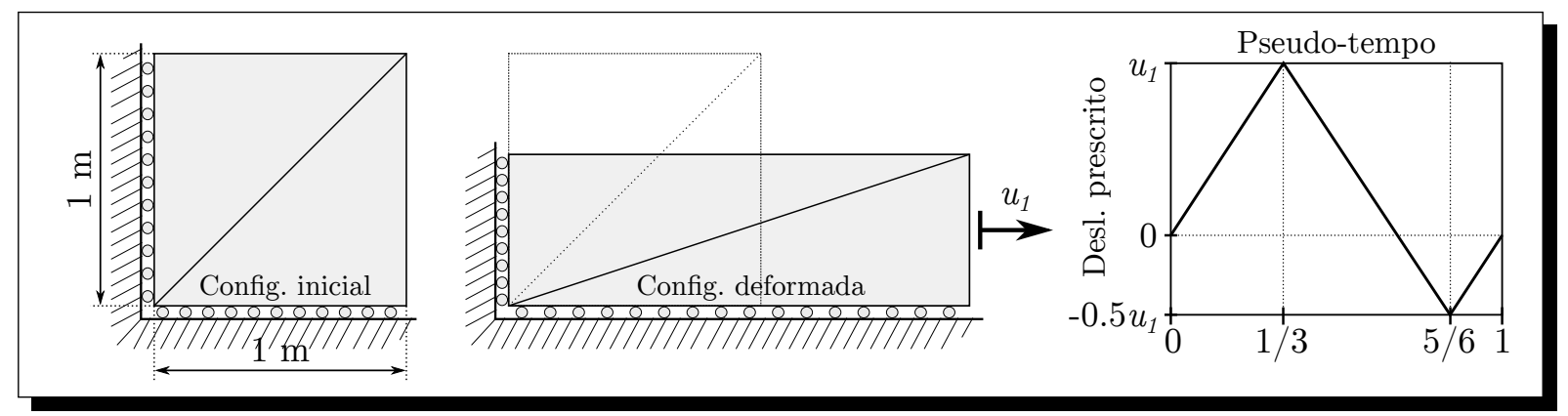

Uma vez que a tensão é uniaxial, considera-se nesse exemplo o estado plano de tensões. De forma a obter campos de tensão e deformação constantes ao longo do domínio, adotam-se apenas 2 elementos finitos de 3 nós e ordem linear. Utiliza-se apenas 1 ponto de integração em cada elemento, onde são medidas as variáveis de interesse.

Na Figura 19 são traçados os gráficos de tensão-deformação linear para cada material considerado. A tensão de Cauchy foi obtida conforme a equação (2.41), e a deformação linear nesse caso é dada por $u / L$, onde $L$ é o comprimento inicial da barra e $u$ é valor do deslocamento prescrito. Os gráficos foram comparados com os de Dettmer e Reese (2004), mostrando resultados satisfatórios e atestando a validade do código desenvolvido, em especial no que diz respeito ao EPT, uma vez que a fonte utilizou o modelo constitutivo completo com elementos tridimensionais.

Figura 19 - Diagramas tensão-deformação para o exemplo de tensão uniaxial

(a) Material metálico

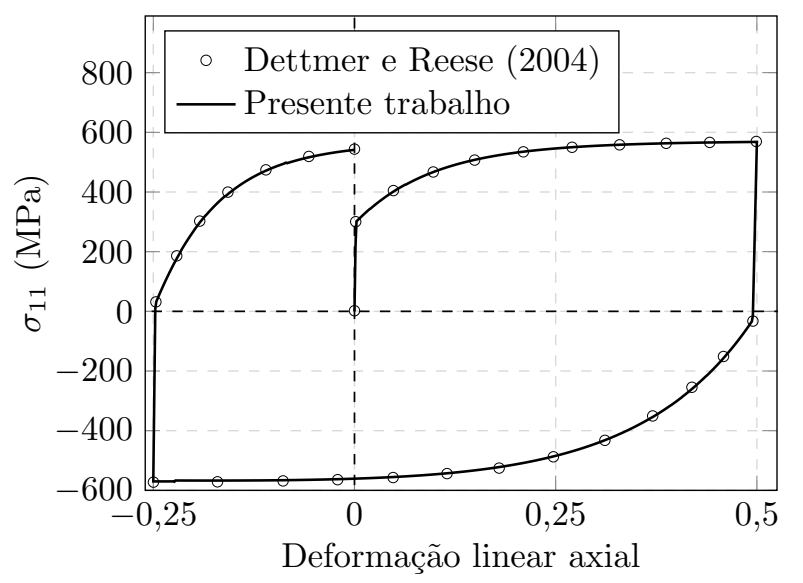

(b) Material polimérico

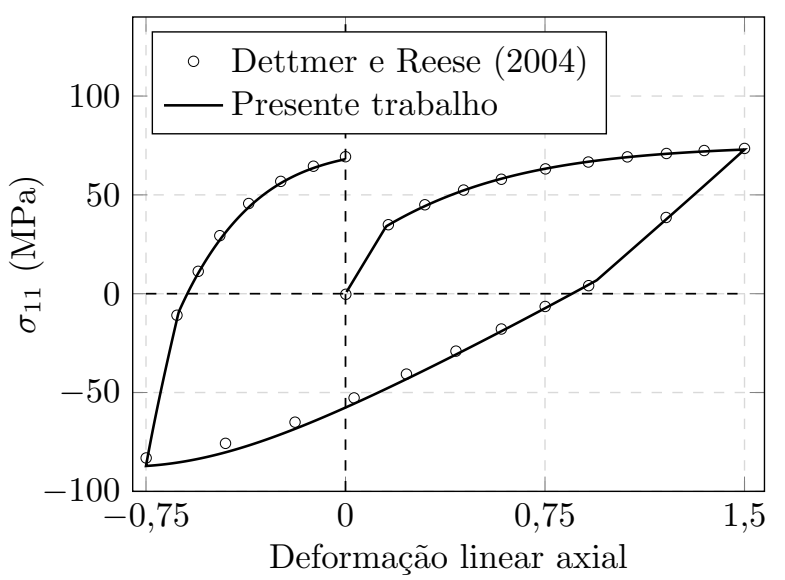




\subsubsection{Chapa sob cisalhamento simples}

No segundo exemplo, pretende-se obter a relação tensão-deformação de um sólido sujeito a cisalhamento simples. Considera-se uma chapa com dimensões e malha equivalentes à do exemplo anterior e estado plano de deformações. Na face inferior, os deslocamentos são totalmente restritos. $\mathrm{Na}$ face superior, restringe-se verticalmente e aplica-se deslocamento horizontal prescrito conforme o gráfico da Figura 20, com intensidade máxima $u_{1}=1$ $\mathrm{m}$ para o material metálico e $u_{1}=4 \mathrm{~m}$ para material polimérico. Novamente, foram considerados 1200 passos.

Figura 20 - Chapa sob cisalhamento simples

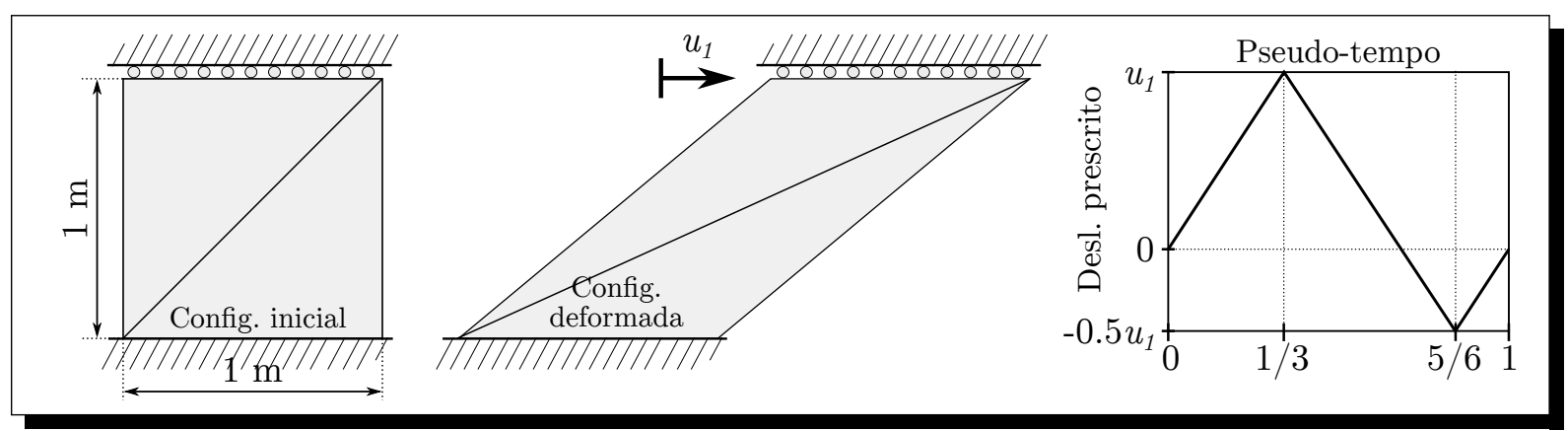

Figura 21 - Diagramas tensão-deformação para o exemplo de deformação cisalhante simples

(a) Material metálico

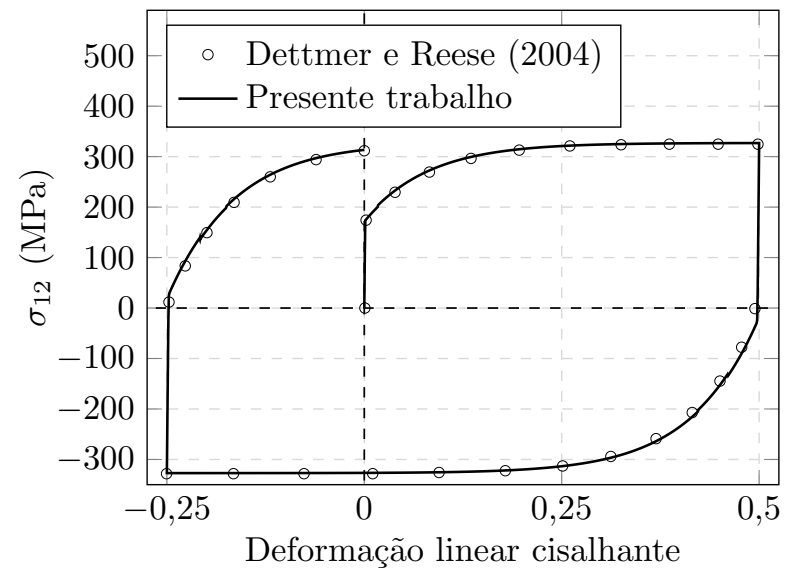

(b) Material polimérico

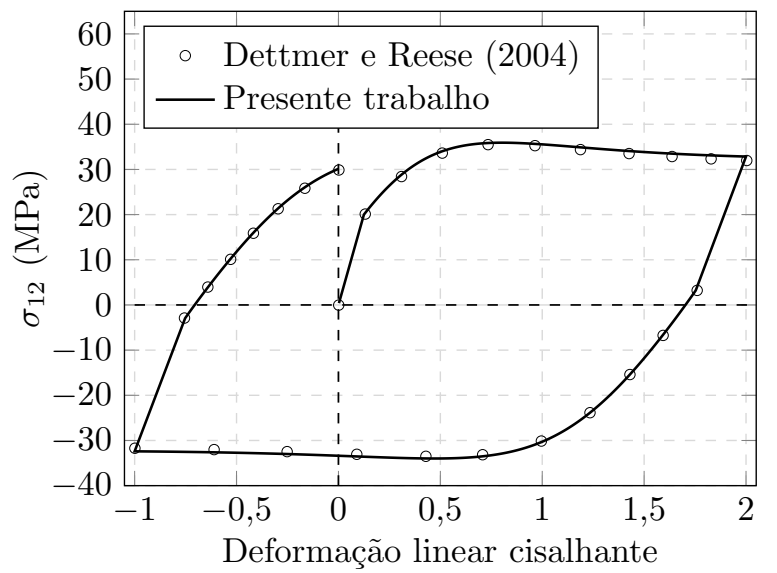

Os gráficos de tensão-deformação para cada material são mostrados na Figura 21, e comparados com os de Dettmer e Reese (2004), mostrando resultados condizentes. Novamente, a tensão de Cauchy foi obtida conforme a equação (2.41), e a deformação linear nesse caso é dada por $u / 2 h$, onde $h$ é a altura inicial da barra e $u$ é valor do deslocamento prescrito.

\subsection{Modelo visco-plástico de Perzyna}

Conforme mencionado na introdução deste capítulo, modelos denominados viscoplásticos diferenciam-se de elasto-plásticos por apresentarem dependência temporal, sendo influenciados diretamente pelas taxas de deformação. Nesse contexto, um dos modelos 
mais utilizados na literatura é o de Perzyna (PERZYNA, 1966), que caracteriza-se por substituir o método dos multiplicadores de Lagrange (ver subseção 4.1.6) pelo método da penalização para impor o critério de plastificação $(\Phi \leq 0)$. Tal critério, por sua vez, pode ser reescrito como $\langle\Phi\rangle=0$, onde $\langle\cdot\rangle$ denota os colchetes de Macauley:

$$
\langle\Phi\rangle=\frac{1}{2}(\Phi+|\Phi|) .
$$

Dessa forma, pelo princípio da máxima dissipação de energia, deve-se minimizar o funcional

$$
\ell\left(\mathbf{M}^{e}-\boldsymbol{\chi}, \mathbf{M}_{e}^{p}, \sigma_{\kappa}\right)=-\left(\mathbf{M}^{e}-\chi\right): \mathbf{L}^{p}-\mathbf{M}_{e}^{p}: \mathbf{L}_{i}^{p}+\sigma_{\kappa} \dot{\kappa}+\frac{1}{2 \eta_{p}}\langle\Phi\rangle^{2},
$$

onde $\eta_{p}$ representa o parâmetro de viscosidade. Naturalmente, quanto menor o valor de $\eta_{p}$, maior será o coeficiente de penalização $1 / 2 \eta_{p}$, e, portanto, mais próximo o modelo será do elasto-plástico.

\subsubsection{Leis de evolução}

Minimizando (4.108) com relação à $\mathbf{M}^{e}-\boldsymbol{\chi}, \mathbf{M}_{e}^{p}$ e $\sigma_{\kappa}$, pode-se obter, respectivamente, as seguintes leis de evolução:

$$
\left\{\begin{aligned}
\mathbf{L}^{p}=\frac{\langle\Phi\rangle}{\eta_{p}} \mathbf{N}^{p}, & \text { onde } \mathbf{N}^{p}=\frac{\partial\langle\Phi\rangle}{\partial\left(\mathbf{M}^{e}-\boldsymbol{\chi}\right)} \\
\mathbf{L}_{i}^{p}=\frac{\langle\Phi\rangle}{\eta_{p}} \mathbf{N}_{i}^{p}, & \text { onde } \mathbf{N}_{i}^{p}=\frac{\partial\langle\Phi\rangle}{\partial \mathbf{M}_{e}^{p}} \\
\dot{\kappa}=\frac{\langle\Phi\rangle}{\eta_{p}} n_{\kappa}, & \text { onde } n_{\kappa}=-\frac{\partial\langle\Phi\rangle}{\partial \sigma_{\kappa}} .
\end{aligned}\right.
$$

Seja agora uma lei de potencial plástico $\Omega$, que pode ser tomada conforme a equação (4.82), e uma função $\Theta$ tal que:

$$
\left\{\begin{aligned}
\mathbf{L}^{p}=\frac{\langle\Theta\rangle}{\eta_{p}} \mathbf{N}^{p}, & \text { onde } \mathbf{N}^{p}=\frac{\partial\langle\Omega\rangle}{\partial\left(\mathbf{M}^{e}-\chi\right)} \\
\mathbf{L}_{i}^{p}=\frac{\langle\Theta\rangle}{\eta_{p}} \mathbf{N}_{i}^{p}, & \text { onde } \mathbf{N}_{i}^{p}=\frac{\partial\langle\Omega\rangle}{\partial \mathbf{M}_{e}^{p}} \\
\dot{\kappa}=\frac{\langle\Theta\rangle}{\eta_{p}} n_{\kappa}, & \text { onde } n_{\kappa}=-\frac{\partial\langle\Omega\rangle}{\partial \sigma_{\kappa}} .
\end{aligned}\right.
$$

A função $\Theta$ é denominada função das tensões excedentes (over-stress), dependente apenas do critério de plastificação $\Phi$, e escolhida de forma que seja contínua e convexa em $[0, \infty)$, e nula quando $\Phi=0$ (HEERES; SUIKER; BORST, 2002). Seguindo essas condições, pode-se definir a lei de Norton (LEMAITRE, 2001),

$$
\Theta=\left(\frac{\Phi}{\alpha_{p}}\right)^{m},
$$

onde o valor de $\alpha_{p}$ é muitas vezes tomado como a tensão de escoamento inicial, e $m$ é um coeficiente adimensional que controla a sensibilidade do modelo com relação à taxa, devendo ser sempre maior ou igual a 1 para que seja atendida a condição de convexidade. 
Dessa forma, pode-se manter as leis de evolução (4.86), (4.87) e (4.88) do modelo elasto-plástico, porém, calcula-se o multiplicador plástico $\dot{\gamma}$ de forma explícita pela equação:

$$
\dot{\gamma}=\frac{1}{\eta_{p}}\left\langle\left(\frac{\Phi}{\alpha_{p}}\right)^{m}\right\rangle .
$$

Como consequência do método da penalização, o valor de $\Phi$ poderá ser maior que zero. Logo, as condições de complementaridade e consistência (subseção 4.1.7) não são aplicáveis ao modelo visco-plástico de Perzyna. Dessa forma, para garantir que $\dot{\gamma}=0$ quando $\Phi<0$, o uso dos colchetes de Macauley na equação (4.112) é indispensável.

\subsubsection{Aplicação de algoritmo implícito}

De forma análoga às equações (4.101)-(4.103), a aplicação do algoritmo implícito de Euler resulta na seguinte forma para as leis de evolução:

$$
\begin{gathered}
\Delta \mathbf{A}^{p}=\mathbf{A}_{s+1}^{p}-\mathbf{A}_{s}^{p}=\Delta \gamma_{s+1} \mathbf{N}^{p} \mathbf{A}^{p}, \\
\Delta \kappa=\kappa_{s+1}-\kappa_{s}=\Delta \gamma_{s+1} \sqrt{2 / 3} \mathrm{e} \\
\Delta \mathbf{A}_{i}^{p}=\left(\mathbf{A}_{i}^{p}\right)_{s+1}-\left(\mathbf{A}_{i}^{p}\right)_{s}=\Delta \gamma_{s+1} \mathbf{N}_{i}^{p} \mathbf{A}_{i}^{p},
\end{gathered}
$$

onde

$$
\frac{\Delta \gamma_{s+1}}{\Delta t}=\frac{1}{\eta_{p}}\left\langle\left(\frac{\Phi_{s+1}}{\alpha_{p}}\right)^{m}\right\rangle .
$$

Dessa maneira, o problema consiste em resolver, para as variáveis $\mathbf{A}_{s+1}^{p}, \kappa_{s+1}$, $\left(\mathbf{A}_{i}^{p}\right)_{s+1}$ e $\Delta \gamma_{s+1}$, o seguinte sistema não-linear:

$$
\left\{\begin{array}{l}
\mathbf{R}_{1}=\mathbf{A}_{s+1}^{p}-\mathbf{A}_{s}^{p}-\Delta \gamma_{s+1} \mathbf{N}_{s+1}^{p} \mathbf{A}_{s+1}^{p}=\mathbf{0} \\
r_{2}=\kappa_{s+1}-\kappa_{s}-\Delta \gamma_{s+1}\left(r_{\kappa}\right)=0 \\
\mathbf{R}_{3}=\left(\mathbf{A}_{i}^{p}\right)_{s+1}-\left(\mathbf{A}_{i}^{p}\right)_{s}-\Delta \gamma_{s+1} \mathbf{N}_{i}^{p} \mathbf{A}_{i}^{p}=\mathbf{0} \\
r_{4}=\Delta \gamma_{s+1}-\frac{\Delta t}{\eta_{p}}\left\langle\left(\frac{\Phi_{s+1}}{\alpha_{p}}\right)^{m}\right\rangle=0
\end{array}\right.
$$

análogo ao sistema (4.105), com a única diferença que a condição de Kuhn-Tucker, $\Phi=0$, é substituída pela forma explícita de $\Delta \gamma_{s+1}$. Novamente, o sistema é resolvido neste trabalho pelo método de Newton-Raphson.

\subsubsection{Operador tangente consistente visco-plástico}

O operador tangente consistente do modelo visco-plástico é dado ainda pela equação (4.95). Entretanto, o cálculo das derivadas auxiliares difere-se pelo fato de que, no caso elasto-plástico, parte-se da condição de complementaridade $\dot{\Phi}=0$, que não é válida no modelo visco-plástico. No entanto, tal condição pode ser substituída por $\Delta r_{4}=0$, uma vez que $r_{4}=0$ para todos os passos de tempo, conforme estabelecido pelo sistema (4.117). Dessa forma, aplica-se a aproximação 


$$
\begin{aligned}
\Delta r_{4}=0 & \approx \frac{\partial r_{4}}{\partial \mathbf{E}}: \Delta \mathbf{E}+\frac{\partial r_{4}}{\partial \mathbf{A}^{p}}: \Delta \mathbf{A}^{p}+\frac{\partial r_{4}}{\partial \kappa} \Delta \kappa+\frac{\partial r_{4}}{\partial \mathbf{A}_{i}^{p}}: \Delta \mathbf{A}_{i}^{p}+\frac{\partial r_{4}}{\partial \gamma} \Delta \gamma \\
& \approx \frac{\partial r_{4}}{\partial \mathbf{E}}: \Delta \mathbf{E}+\Delta \gamma\left(\frac{\partial r_{4}}{\partial \mathbf{A}^{p}}:\left(\mathbf{N}^{p} \mathbf{A}^{p}\right)+\frac{\partial r_{4}}{\partial \kappa} \sqrt{\frac{2}{3}}+\frac{\partial r_{4}}{\partial \mathbf{A}_{i}^{p}}:\left(\mathbf{N}_{i}^{p} \mathbf{A}_{i}^{p}\right)+1\right),
\end{aligned}
$$

que resulta em

$$
\Delta \gamma \approx \mathbf{D}^{\gamma}: \Delta \mathbf{E}
$$

onde

$$
\mathbf{D}^{\gamma}=-\frac{\partial r_{4} / \partial \mathbf{E}}{\frac{\partial r_{4}}{\partial \mathbf{A}^{p}}:\left(\mathbf{N}^{p} \mathbf{A}^{p}\right)+\frac{\partial r_{4}}{\partial \mathbf{A}_{i}^{p}}:\left(\mathbf{N}_{i}^{p} \mathbf{A}_{i}^{p}\right)+\frac{\partial r_{4}}{\partial \kappa} \sqrt{\frac{2}{3}}+1},
$$

e, pela lei de Norton,

$$
\frac{\partial r_{4}}{\partial(\bullet)}=-\frac{m \Delta t}{\eta_{p} \alpha_{p}}\left\langle\left(\frac{\Phi}{\alpha_{p}}\right)^{m-1} \frac{\partial \Phi}{\partial(\bullet)}\right\rangle,
$$

sendo (•) utilizado para representar as variáveis $\mathbf{E}, \mathbf{A}^{p}, \mathbf{A}_{i}^{p}$ ou $\kappa$.

Aplicando-se (4.119) em (4.113), resulta:

$$
\Delta \mathbf{A}^{p}=\Delta \gamma \mathbf{N}^{p} \mathbf{A}^{p} \approx\left(\mathbf{D}^{\gamma}: \Delta \mathbf{E}\right) \mathbf{N}^{p} \mathbf{A}^{p}=\left[\left(\mathbf{N}^{p} \mathbf{A}^{p}\right) \otimes \mathbf{D}^{\gamma}\right]: \Delta \mathbf{E}
$$

que, para intervalos de tempo suficientemente pequenos, resulta na equação (4.100), isto é:

$$
\frac{d \mathbf{A}^{p}}{d \mathbf{E}}=\left(\mathbf{N}^{p} \mathbf{A}^{p}\right) \otimes \mathbf{D}^{\gamma}
$$

Observa-se, portanto, que o operador tangente consistente visco-plástico calculado neste trabalho é aproximado, sendo mais próximo do exato quanto mais refinada for a discretização temporal.

\subsubsection{Exemplos numéricos}

Com o intuito de caracterizar o modelo visco-plástico adotado, apresentam-se nesta seção os exemplos numéricos clássicos de chapa sob tensão uniaxial e cisalhamento simples. Na subseção 4.3.4.1 é realizada ainda uma verificação com o trabalho de Heeres, Suiker e Borst (2002), onde o modelo de Perzyna foi aplicado ao caso de pequenas deformações. Apesar do modelo exibir dependência temporal, despreza-se a massa dos materiais considerados, o que permite uma análise quase-estática em todos os casos.

\subsubsection{Chapa sob cisalhamento simples em pequenas deformações}

Considera-se neste exemplo a mesma geometria e malha dadas na Figura 20, e material visco-plástico com modelo de Perzyna e lei de Norton. Por se tratar de um problema com pequenas deformações, utilizou-se a lei de Saint Venant-Kirchhoff para a parcela elástica. Na parcela plástica, foi considerado apenas encruamento isotrópico, com a lei polinomial apresentada na Tabela 1 , onde utilizou-se $n=1$, isto é,

$$
\sigma_{\kappa}(\kappa)=a_{0}+a_{1} \kappa .
$$


Deve ser ressaltado que, para que haja equivalência entre a formulação adotada no presente trabalho e a de Heeres, Suiker e Borst (2002), o critério de plastificação é modificado para a seguinte expressão

$$
\Phi^{*}\left(\mathbf{M}^{e}, \boldsymbol{\chi}, \kappa\right)=\sqrt{\frac{3}{2}}\left\|\left(\mathbf{M}^{e}-\chi\right)^{D}\right\|-\sigma_{\kappa}(\kappa) \leq 0,
$$

isto é, multiplica-se $\sqrt{3 / 2}$ no critério da equação 4.74, adotado originalmente. Na prática, essa diferença pode ser contornada adotando-se uma viscosidade $\eta_{p}=\left(\sqrt{\frac{2}{3}}\right)^{m+1} \eta_{p}^{*}$, onde $\eta_{p}^{*}$ é a viscosidade adotada para o critério (4.125). De fato, pode-se demonstrar facilmente que tal consideração garante a equivalência das leis de evolução, isto é:

$$
\mathbf{L}^{p}=\frac{1}{\eta_{p}^{*}}\left\langle\left(\frac{\Phi^{*}}{\alpha_{p}}\right)^{m}\right\rangle \frac{\partial\left\langle\Phi^{*}\right\rangle}{\partial\left(\mathbf{M}^{e}-\boldsymbol{\chi}\right)}=\frac{1}{\eta_{p}}\left\langle\left(\frac{\Phi}{\alpha_{p}}\right)^{m}\right\rangle \frac{\partial\langle\Phi\rangle}{\partial\left(\mathbf{M}^{e}-\boldsymbol{\chi}\right)} .
$$

Os parâmetros do material são apresentados na Tabela 3.

Tabela 3 - Parâmetros do material, extraídos de Heeres, Suiker e Borst (2002)

\begin{tabular}{c|c|c|c|c|c|c|c}
\hline$E\left(N / m^{2}\right)$ & $\nu$ & $a_{0}\left(N / m^{2}\right)$ & $a_{1}\left(N / m^{2}\right)$ & $\eta_{p}^{*}(\mathrm{~s})$ & $\eta_{p}(\mathrm{~s})$ & $m$ & $\alpha_{p}\left(N / m^{2}\right)$ \\
\hline $2.0 \cdot 10^{7}$ & 0.2 & $2.0 \cdot 10^{3}$ & $-5.0 \cdot 10^{6}$ & 1.0 & $2 / 3$ & 1 & $2.0 \cdot 10^{3}$ \\
\hline
\end{tabular}

São analisados dois casos. No primeiro, aplica-se deslocamento progressivo de valor máximo $1,174 \cdot 10^{-3} \mathrm{~m}$ a uma taxa de $1 \mathrm{~m} / \mathrm{s}$ em 40 passos de tempo, isto é, aplica-se em cada passo um incremento de tempo $\Delta t=4,35 \cdot 10^{-5}$ s e de deslocamento $\Delta u=4,35 \cdot 10^{-5} \mathrm{~m}$. Já no segundo caso, são realizadas etapas de carregamento e descarregamento. Inicialmente, aplica-se deslocamento de valor de $3,0 \cdot 10^{-4} \mathrm{~m}$ em 17 passos, em seguida $-1,77 \cdot 10^{-4} \mathrm{~m}$ em 10 passos, e, por fim, $5,77 \cdot 10^{-4}$ em 33 passos. Novamente, a taxa considerada é de 1 $\mathrm{m} / \mathrm{s}$, isto é, $\Delta t=1,77 \cdot 10^{-5} \mathrm{~s}$. Ambos os casos são ilustrados na Figura 22.

Figura 22 - Evolução dos deslocamentos para cisalhamento simples em material visco-plástico

(a) Deformação progressiva

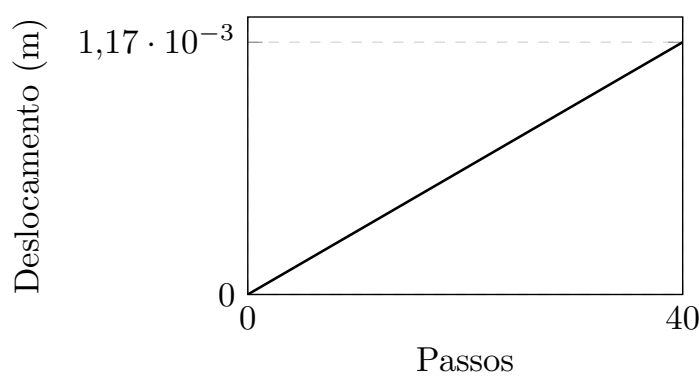

(b) Carregamento e descarregamento

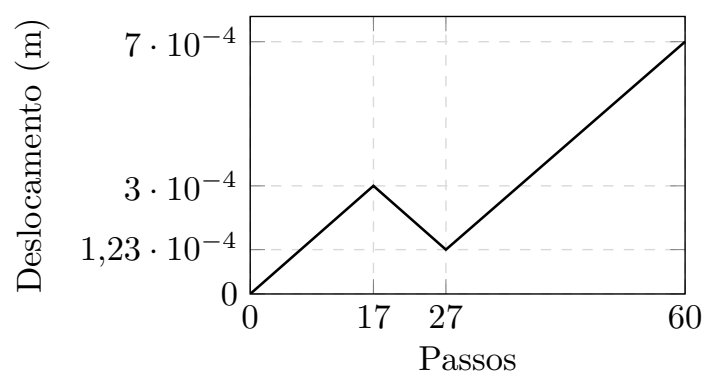

Os resultados são apresentados nas Figuras 23a e 23b, mostrando uma boa conformação com o trabalho de Heeres, Suiker e Borst (2002). Nos dois casos, a tensão de Cauchy foi obtida conforme a equação (2.41), e a deformação linear cisalhante é dada por $u / h$, onde $u$ é o deslocamento aplicado e $h$ é a altura da chapa. 
Figura 23 - Diagramas tensão-deformação para cisalhamento simples em material visco-plástico

(a) Deformação progressiva

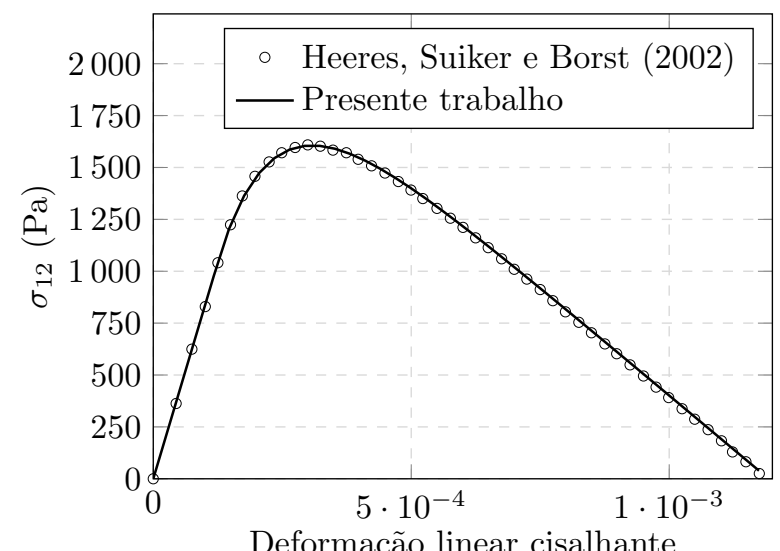

(b) Carregamento e descarregamento

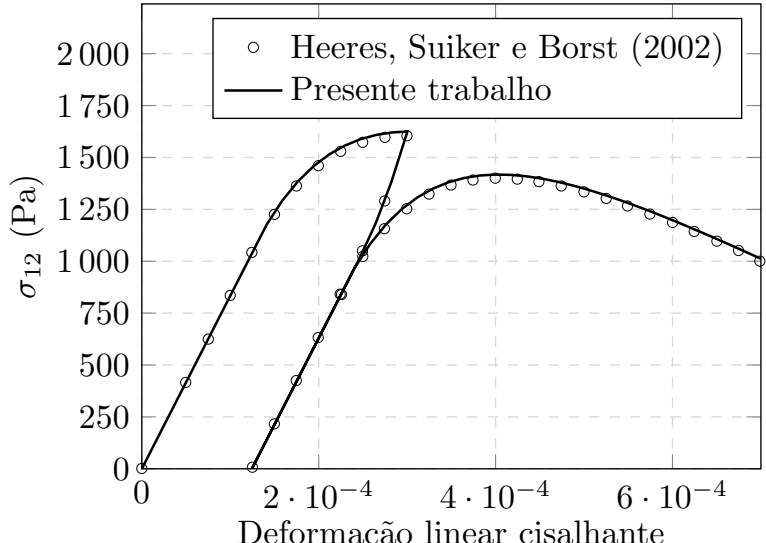

\subsubsection{Chapa sob tensão uniaxial}

Este exemplo tem como objetivo verificar o comportamento de um material viscoplástico em grandes deformações quando submetido a diferentes taxas de deformação. Considera-se uma chapa com geometria e malha semelhantes à da Figura 18, porém, com deslocamento prescrito aplicado na face direita conforme gráfico da Figura 24. O tempo é discretizado em 1000 passos, sendo 500 para a etapa com deslocamento crescente, e 500 para a etapa com deslocamento constante.

Figura 24 - Evolução do deslocamento para chapa visco-plástica sob tensão uniaxial

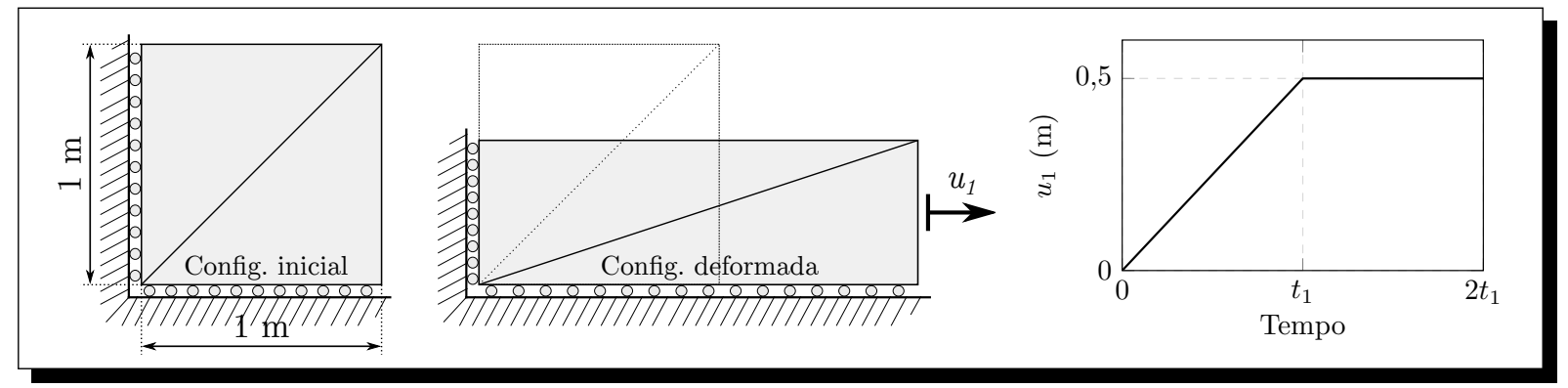

O material adotado é metálico (Mild Steel Ck15), conforme a Tabela 2. Os parâmetros de Perzyna e Norton para esse material, de acordo com Lührs, Hartmann e Haupt (1997), são $\eta_{p}^{*}=10000 \mathrm{~s}, \alpha_{p}^{*}=1 \mathrm{MPa}^{2}$ e $m^{*}=2$. Uma vez que o critério de plastificação adotado por esse trabalho $\left(\Phi^{*}\right)$ não pode ser associado diretamente com o da presente formulação $(\Phi)$, não é possível realizar uma calibragem exata dos parâmetros, como foi feito no exemplo anterior. Entretanto, para garantir pelo menos ordens de grandeza semelhantes, parte-se da aproximação $\Phi^{*}=\frac{1}{2} \Phi^{2}$. Dessa forma, realizando um procedimento análogo ao da equação (4.126), pode-se obter:

$$
\eta_{p}=2^{m^{*}} \eta_{p}^{*}, \quad \alpha_{p}=\sqrt{\alpha_{p}^{*}}, \quad \text { e } \quad m=2 m^{*},
$$

de onde resultam os parâmetros dados na Tabela 4 .

Na Figura 25 apresenta-se o gráfico das tensões de Cauchy do exemplo para 
Tabela 4 - Parâmetros de Perzyna e Norton para o Mild Steel Ck15

\begin{tabular}{c|c|c}
\hline$\eta_{p}(\mathrm{~s})$ & $\alpha_{p}(\mathrm{MPa})$ & $m$ \\
\hline $4 \cdot 10^{4}$ & 1 & 4 \\
\hline
\end{tabular}

diferentes taxas de deslocamento (isto é, diferentes valores de tempo $t_{1}$ ). Observa-se que a redução da taxa provoca um comportamento semelhante à redução do parâmetro de viscosidade, isto é, faz com que a resposta se aproxime do modelo elasto-plástico. Além disso, na etapa de deformação constante, percebe-se para todos os casos uma tendência à resposta elasto-plástica quando o tempo tende a infinito.

\subsubsection{Chapa sob cisalhamento simples}

Neste exemplo, considera-se uma chapa sob cisalhamento simples com dados de geometria e malha semelhantes à Figura 20, porém, com deslocamento prescrito aplicado de acordo com o gráfico da Figura 26. Neste, $t_{1}$ é variável, e adotam-se 1600 passos.

O material, neste caso, é o polimérico (PET orientado) da Tabela 2, com a adição dos parâmetros de Perzyna e Norton dados na Tabela 5.

Tabela 5 - Parâmetros de Perzyna e Norton para o material polimérico

\begin{tabular}{c|c|c}
\hline$\eta_{p}(\mathrm{~s})$ & $\alpha_{p}(\mathrm{MPa})$ & $m$ \\
\hline 1 & 35 & 1 \\
\hline
\end{tabular}

Os gráficos de tensão em função da componente $A_{12}$ são apresentados na Figura 27 para diversos valores de $t_{1}$. Nas figuras 28 e 29 apresentam-se os valores de tensões e de $\mathbf{A}^{p}$ ao longo do tempo para cada $t_{1}$ considerado. É interessante observar que, neste caso, o efeito de Bauschinger provoca comportamentos distintos quando aplicadas taxas de deformações muito grandes, uma vez que, para os casos $t_{1}=0,5 \mathrm{~s}$ e $t_{1}=0,1 \mathrm{~s}$, as tensões $\sigma_{12}$ e os valores de $A_{12}^{p}$ e $A_{21}^{p}$ possuem sinais contrários aos demais casos, indicando que a

Figura 25 - Tensões para chapa visco-plástica sob tensão uniaxial

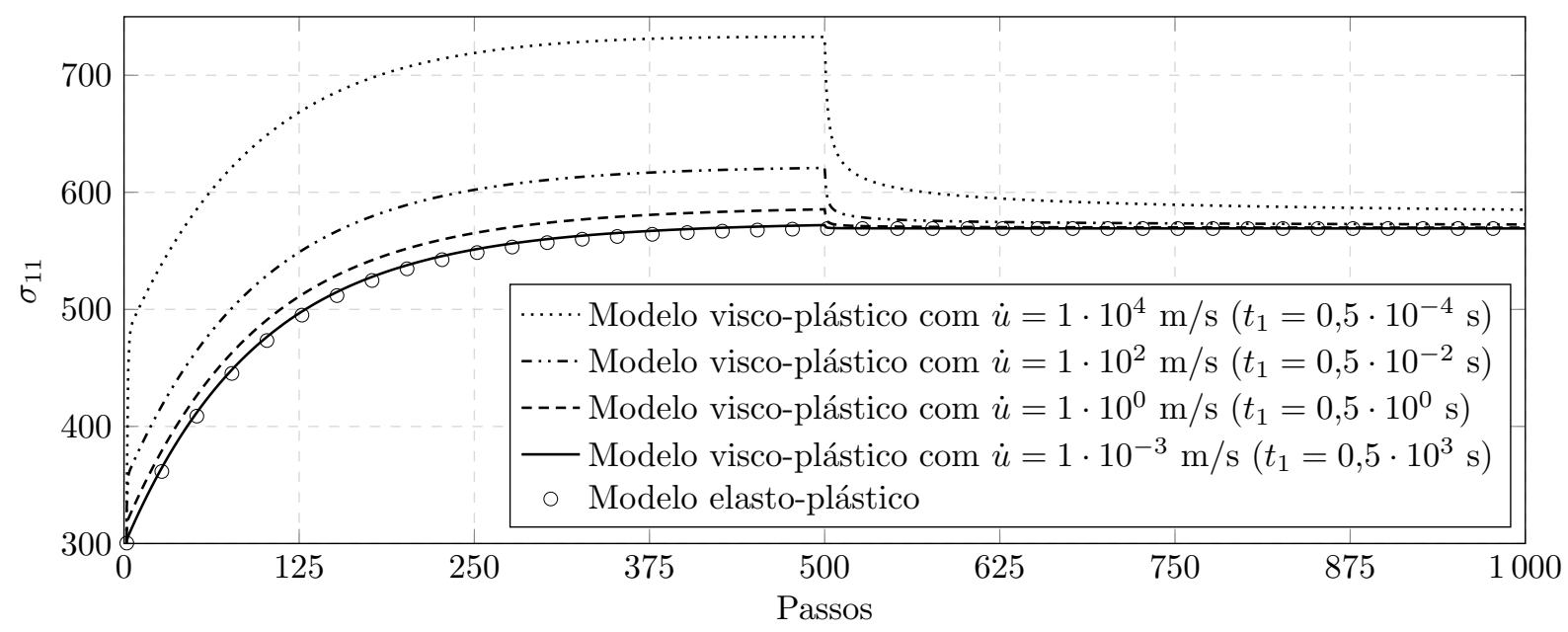


Figura 26 - Deslocamento prescrito em chapa visco-plástica sob cisalhamento simples

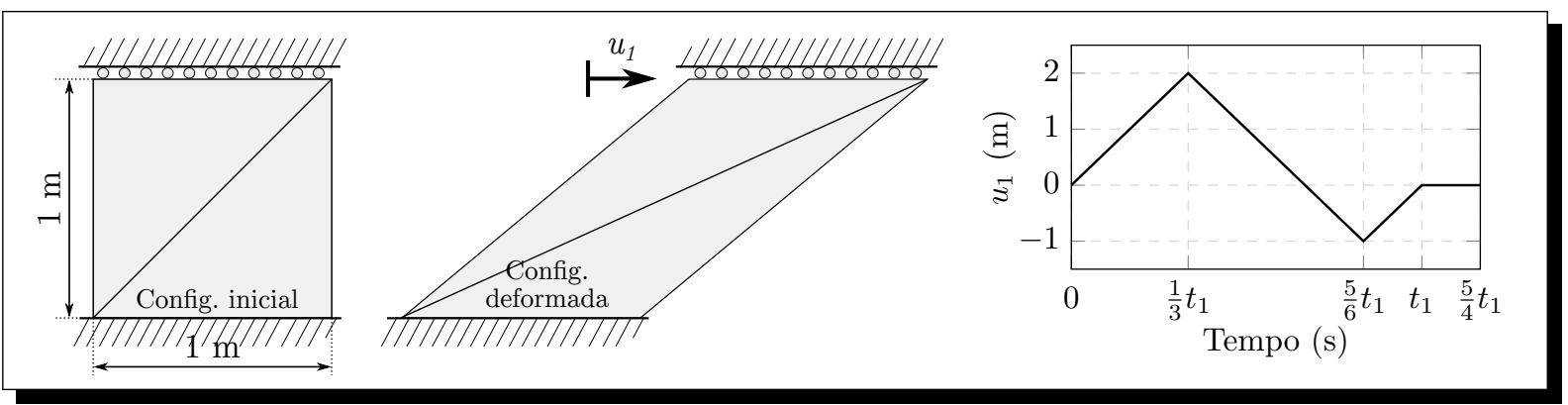

Figura 27 - Deformação-tensão para chapa visco-plástica sob cisalhamento simples

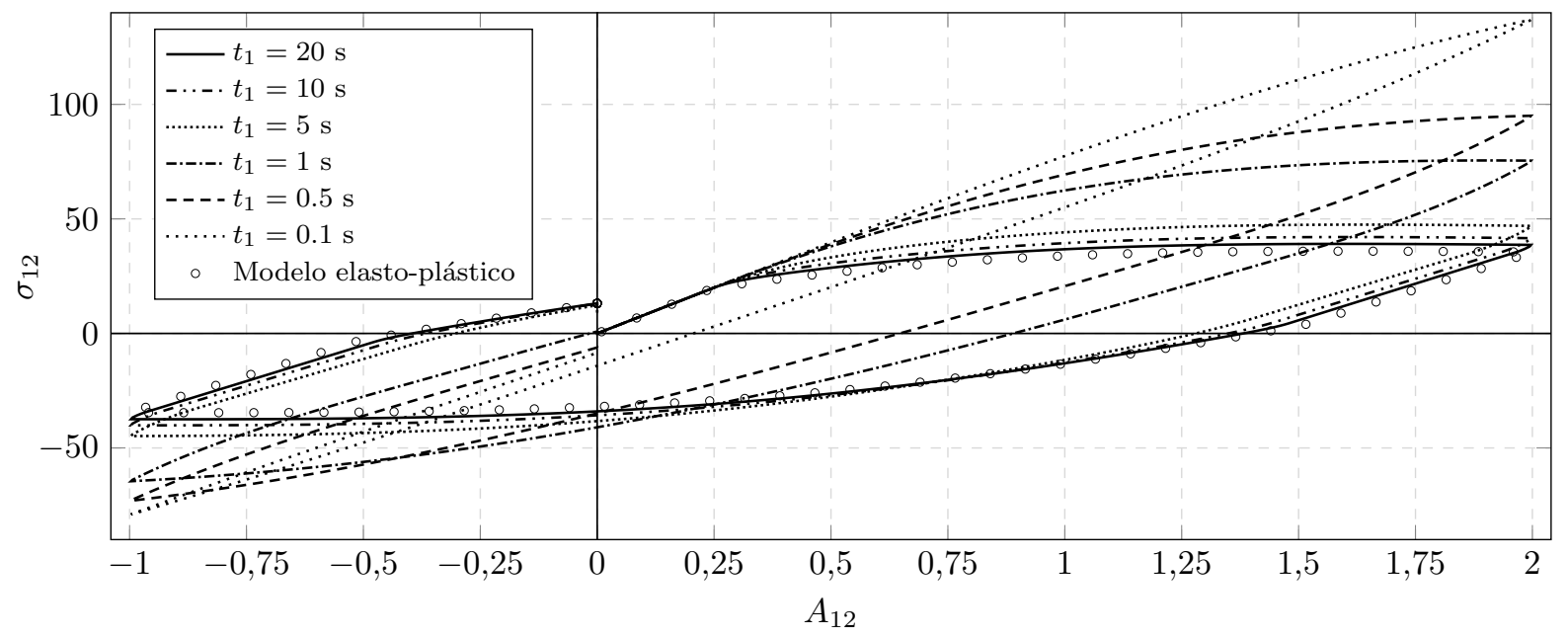

Figura 28 - Tensões de Cauchy ao longo do tempo para chapa visco-plástica sob cisalhamento simples
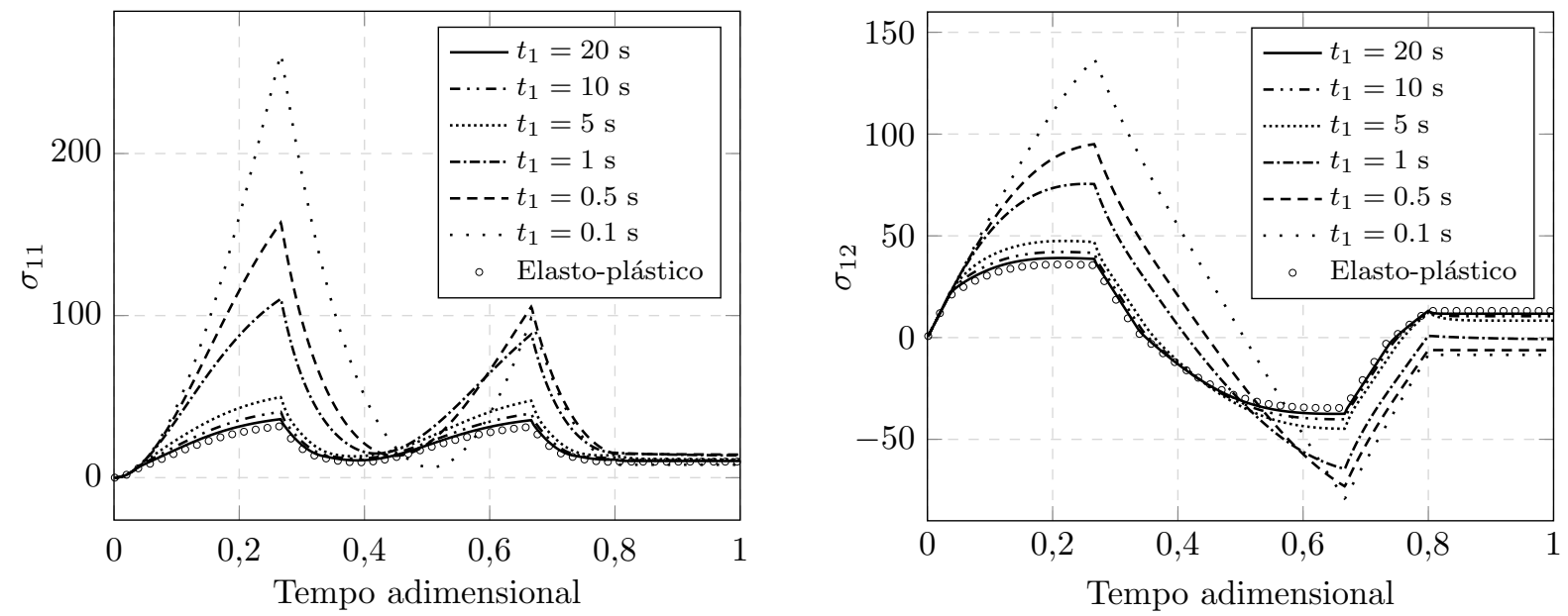

chapa possui tendência a deformar nas direções opostas caso tenha suas restrições soltas.

De fato, a partir dos valores de $\mathbf{A}^{p}$ obtidos na Figura 29 é possível definir as configurações intermediárias plásticas no último passo de tempo. Essas configurações são mostradas na Figura 30 para alguns casos de $t_{1}$, onde é possível observar o fenômeno comentado anteriormente: valores muito pequenos e muito grandes de taxas apresentam deformações plásticas em direções opostas. 
Figura 29 - Deformações cisalhantes plásticas ao longo do tempo para chapa visco-plástica sob cisalhamento simples
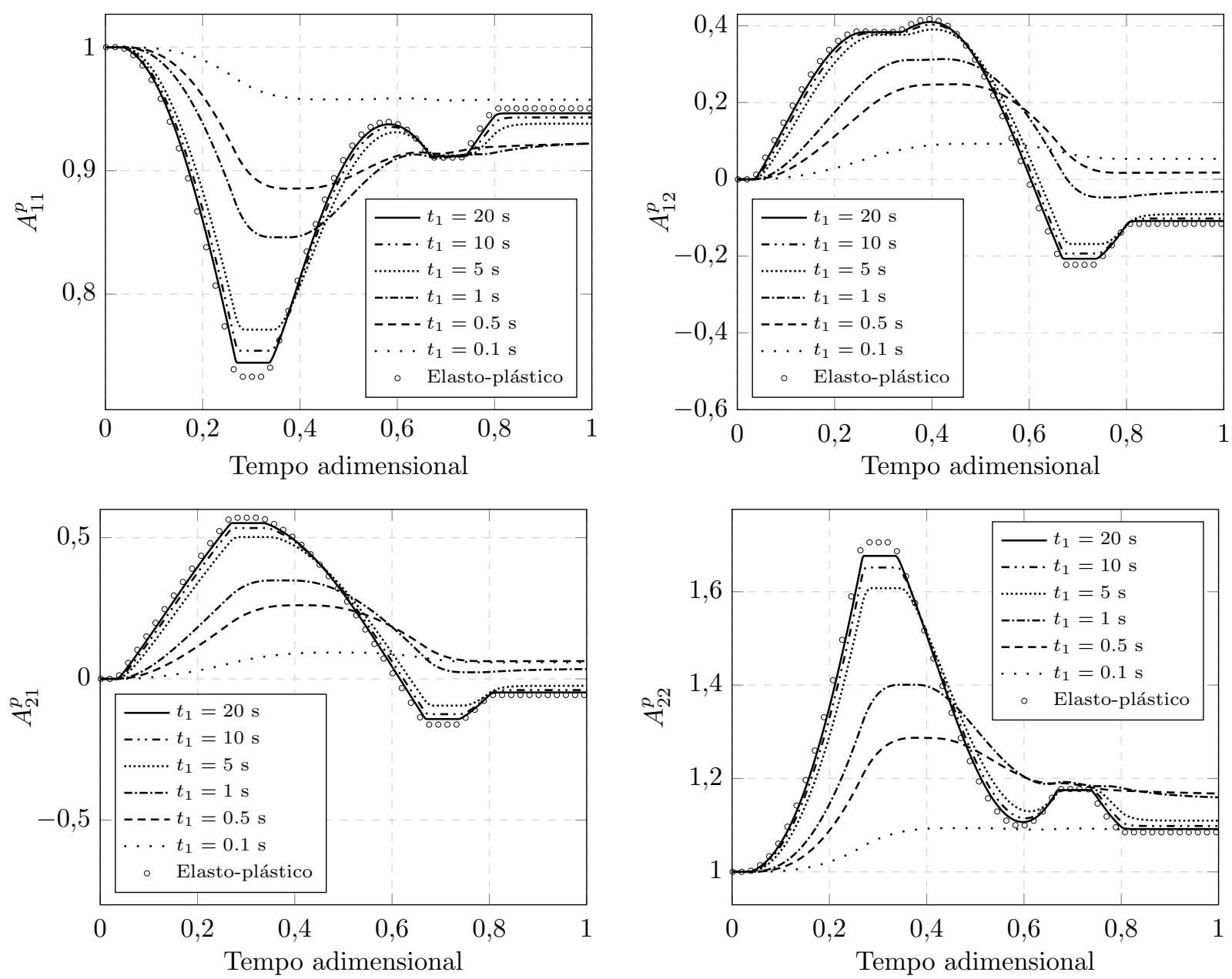

Figura 30 - Configurações intermediárias finais para chapa visco-plástica sob cisalhamento simples

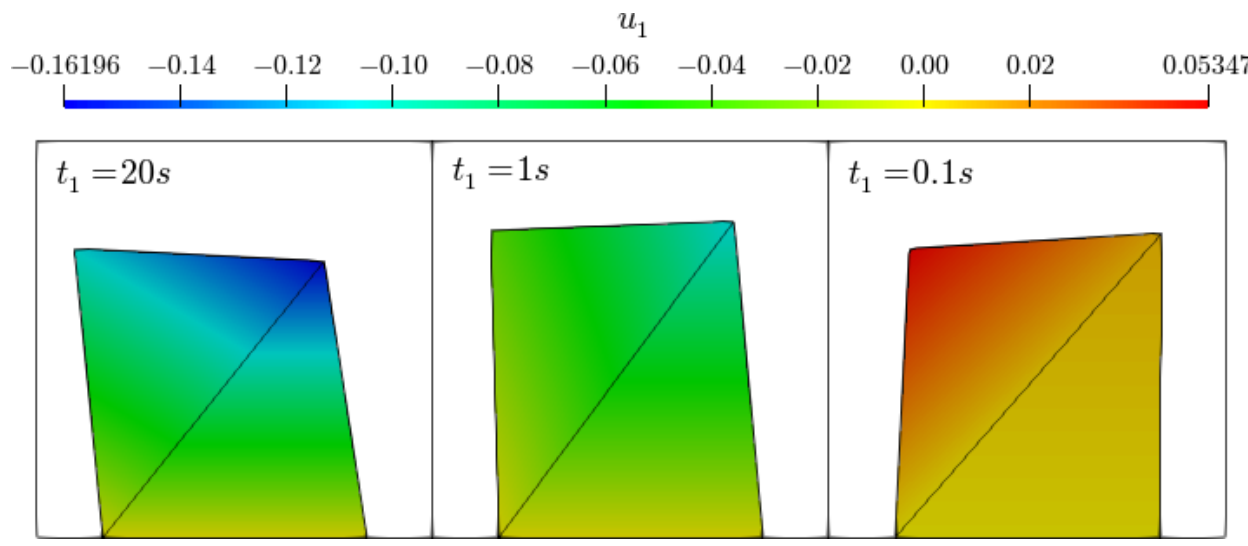





\section{Capítulo 5}

\section{Modelo visco-elástico}

Materiais visco-elásticos possuem a capacidade de simultaneamente armazenar e dissipar energia. De acordo com Lemaitre (2001), a parcela dissipativa se deve a atritos internos que podem ocorrer, por exemplo, entre moléculas (no caso de polímeros) ou células (no caso de madeiras). Dessa forma, tais materiais caracterizam-se pela sua dependência temporal, apresentando respostas que dependem diretamente das taxas de deformações aplicadas.

Uma forma alternativa de caracterizar o modelo visco-elástico é pelo seu comportamento quando submetido a tensões aplicadas subitamente e constantes ao longo do tempo (ensaios de fluência). Enquanto materiais puramente elásticos respondem instantaneamente com um estado de deformações constante ao longo do tempo, materiais visco-elásticos continuam deformando-se, podendo tender a um estado de equilíbrio limitado em magnitude ou não (CHRISTENSEN, 2013).

O modelo visco-elástico descrito neste capítulo parte da energia de dissipação e é válido para o regime de grandes deformações, sendo baseado no trabalho de Pascon e Coda (2017). Outras referências importantes para a compreensão e para o desenvolvimento do modelo são Reese e Govindjee (1997), Huber e Tsakmakis (2000), Hasanpour, Ziaei-Rad e Mahzoon (2009) e Petiteau et al. (2013).

\subsection{Cinemática}

Motivado pela decomposição multiplicativa da equação (4.32), pode-se expressar a função mudança de configuração como

$$
\mathbf{A}=\mathbf{A}^{e} \mathbf{A}^{v}
$$

onde $\mathbf{A}^{e}$ representa a parcela puramente elástica do gradiente da função mudança de configuração, e $\mathbf{A}^{v}$ a parcela puramente viscosa. A configuração intermediária, neste caso denotada por $\Omega^{v}$, representa o estado do sólido em uma situação teórica onde há apenas deformações viscosas. De acordo com Huber e Tsakmakis (2000), tal configuração é obtida se o corpo for descarregado para um determinado estado de equilíbrio em um processo 
infinitamente rápido (no qual $\mathbf{A}^{v}$ é fixo). Mais detalhes sobre o equilíbrio da configuração intermediária são fornecidos na seção 5.2.

Vale ser mencionado que a decomposição (5.1) é questionada por Petiteau et al. (2013), pois, de acordo com o autor, a configuração intermediária só pode ser considerada de fato equilibrada se a escala do fenômeno for muito menor que o tempo de relaxamento.

Uma vez que a decomposição (5.1) é semelhante à (4.32), as definições apresentadas nesta seção serão similares às do modelo elasto-plástico. Isso inclui as deformações

$$
\begin{aligned}
& \mathbf{E}^{e}=\frac{1}{2}\left(\left(\mathbf{A}^{e}\right)^{T} \mathbf{A}^{e}-\mathbf{I}\right)=\frac{1}{2}\left(\mathbf{C}^{e}-\mathbf{I}\right), \quad \mathrm{e} \\
& \mathbf{E}^{v}=\frac{1}{2}\left(\left(\mathbf{A}^{v}\right)^{T} \mathbf{A}^{v}-\mathbf{I}\right)=\frac{1}{2}\left(\mathbf{C}^{v}-\mathbf{I}\right),
\end{aligned}
$$

e os gradientes de velocidade

$$
\begin{aligned}
& \mathbf{L}^{e}=\dot{\mathbf{A}}^{e}\left(\mathbf{A}^{e}\right)^{-1}, \quad \mathrm{e} \\
& \mathbf{L}^{v}=\dot{\mathbf{A}}^{v}\left(\mathbf{A}^{v}\right)^{-1} .
\end{aligned}
$$

Além disso, todas as relações obtidas nas subseções 4.2.1.2 e 4.2.1.3 podem ser aproveitadas no presente contexto, substituindo-se os índices $(\cdot)^{p}$ por $(\cdot)^{v}$, de forma que escreve-se:

$$
\begin{gathered}
\mathbf{C}^{e}=\left(\mathbf{A}^{v}\right)^{-T} \mathbf{C}\left(\mathbf{A}^{v}\right)^{-1}, \\
\mathbf{E}^{e}=\left(\mathbf{A}^{v}\right)^{-T}\left(\mathbf{E}-\mathbf{E}^{v}\right)\left(\mathbf{A}^{v}\right)^{-1}, \\
\mathbf{L}=\dot{\mathbf{A}} \mathbf{A}^{-1}=\mathbf{L}^{e}+\mathbf{A}^{e} \mathbf{L}^{v}\left(\mathbf{A}^{e}\right)^{-1}, \quad \mathrm{e} \\
\dot{\mathbf{E}}^{e}=\left(\mathbf{A}^{v}\right)^{-T} \dot{\mathbf{E}}\left(\mathbf{A}^{v}\right)^{-1}-\frac{1}{2} \mathbf{C}^{e} \mathbf{L}^{v}-\frac{1}{2}\left(\mathbf{L}^{v}\right)^{T} \mathbf{C}^{e} .
\end{gathered}
$$

\subsection{Energia, tensão e dissipação}

A formulação adotada neste trabalho parte do modelo reológico de Zener (PASCON; CODA, 2017), ilustrado na Figura 31.

Figura 31 - Modelo reológico de Zener, adaptado de Pascon e Coda (2017)

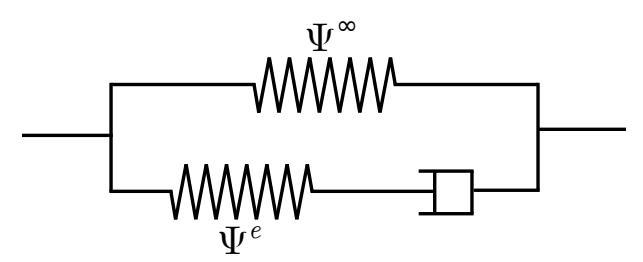

Nesse modelo, a mola associada ao amortecedor possui energia interna denotada por $\Psi^{e}$, dependente apenas da parcela elástica das deformações. Já a mola desassociada do amortecedor, possui energia interna denotada por $\Psi^{\infty}$, e depende das deformações totais. O amortecedor representa as deformações viscosas do material, e seu comportamento ao longo do tempo é manifestado pela lei de evolução do modelo, conforme discutido 
posteriormente. Escreve-se, portanto, a energia livre de Helmholtz como

$$
\Psi\left(\mathbf{E}, \mathbf{E}^{e}\right)=\Psi^{\infty}(\mathbf{E})+\Psi^{e}\left(\mathbf{E}^{e}\right),
$$

de onde derivam as tensões

$$
\mathbf{S}^{\infty}=\frac{\partial \Psi^{\infty}}{\partial \mathbf{E}}=2 \frac{\partial \Psi^{e}}{\partial \mathbf{C}} \quad \text { e } \quad \mathbf{S}^{e}=\frac{\partial \Psi^{e}}{\partial \mathbf{E}^{e}}=2 \frac{\partial \Psi^{e}}{\partial \mathbf{C}^{e}}
$$

e os operadores

$$
\mathfrak{C}^{\infty}=\frac{\partial \mathbf{S}^{\infty}}{\partial \mathbf{E}}=\frac{\partial^{2} \Psi^{\infty}}{\partial \mathbf{E} \otimes \partial \mathbf{E}} \quad \text { e } \quad \mathfrak{C}^{e}=\frac{\partial \mathbf{S}^{e}}{\partial \mathbf{E}^{e}}=\frac{\partial^{2} \Psi^{e}}{\partial \mathbf{E}^{e} \otimes \partial \mathbf{E}^{e}} .
$$

Tomando-se para $\Psi^{\infty}$ e $\Psi^{e}$ a lei hiperelástica neo-Hookeana apresentada na subseção 2.5.2, tem-se

$$
\begin{aligned}
\Psi^{\infty} & =\frac{\Lambda^{\infty}}{2} \ln (J)^{2}+\frac{\mu^{\infty}}{2}[\operatorname{tr}(\mathbf{C})-3-2 \ln (J)], \quad \mathrm{e} \\
\Psi^{e} & =\frac{\Lambda^{e}}{2} \ln \left(J^{e}\right)^{2}+\frac{\mu^{e}}{2}\left[\operatorname{tr}\left(\mathbf{C}^{e}\right)-3-2 \ln \left(J^{e}\right)\right],
\end{aligned}
$$

onde $\Lambda^{\infty}, \mu^{\infty}, \Lambda^{e}$ e $\mu^{e}$ são parâmetros do material e $J^{e}=\operatorname{det}\left(\mathbf{A}^{e}\right)$ é o jacobiano elástico.

Utilizando-se novamente a desigualdade de Clausius-Duhem (4.13) para representar a segunda lei da termodinâmica, calcula-se a energia de dissipação por

$$
d_{i n t}=\mathbf{S}: \dot{\mathbf{E}}-\mathbf{S}^{\infty}: \dot{\mathbf{E}}-\mathbf{S}^{e}: \dot{\mathbf{E}}^{e} \geq 0,
$$

Aplicando (5.9) e seguindo um procedimento análogo ao da equação (4.62), resulta:

$$
\mathbf{S}^{e}: \dot{\mathbf{E}}^{e}=\left(\left(\mathbf{A}^{v}\right)^{-1} \mathbf{S}^{e}\left(\mathbf{A}^{v}\right)^{-T}\right): \dot{\mathbf{E}}-\mathbf{M}^{e}: \mathbf{L}^{v}
$$

onde

$$
\mathbf{M}^{e}=\mathbf{C}^{e} \mathbf{S}^{e}
$$

é o tensor de Mandel, definido, neste caso, na configuração intermediária viscosa. Aplicando (5.16) em (5.15), chega-se a:

$$
d_{i n t}=\left(\mathbf{S}-\mathbf{S}^{\infty}-\left(\mathbf{A}^{v}\right)^{-1} \mathbf{S}^{e}\left(\mathbf{A}^{v}\right)^{-T}\right): \dot{\mathbf{E}}+\mathbf{M}^{e}: \mathbf{L}^{v} \geq 0 .
$$

Para garantir a não-negatividade da energia de dissipação para valores arbitrários de $\dot{\mathbf{E}}$ (COLEMAN; NOLL, 1963), define-se a tensão de Piola-Kirchhoff de segunda espécie como

$$
\mathbf{S}=\mathbf{S}^{\infty}+\left(\mathbf{A}^{v}\right)^{-1} \mathbf{S}^{e}\left(\mathbf{A}^{v}\right)^{-T}=\mathbf{S}^{\infty}+\mathbf{S}^{q}
$$

onde $\mathbf{S}^{q}=\left(\mathbf{A}^{v}\right)^{-1} \mathbf{S}^{e}\left(\mathbf{A}^{v}\right)^{-T}$ é denominada tensão não-equilibrante (PASCON; CODA, 2017). Logo, a energia de dissipação é reduzida para:

$$
d_{i n t}=\mathbf{M}^{e}: \mathbf{L}^{v} \geq 0
$$

e, portanto, o tensor de Mandel, neste caso, é termodinamicamente conjugado à $\mathbf{L}^{v}$.

A expressão (5.19) permite uma nova interpretação para a configuração intermediária viscosa: Suponha que $\mathbf{E}^{e}=\mathbf{0}$. Segue de imediato que $\mathbf{S}^{e}=\mathbf{0}$ e, portanto, a tensão $\mathbf{S}$ se reduz a $\mathbf{S}^{\infty}$. Conclui-se que a configuração intermediária $\Omega^{v}$ é equilibrada por $\mathbf{S}^{\infty}$, 
que, neste caso, é nula se, e somente se, $\mathbf{E}^{v}=\mathbf{0}$. Logo, pode-se obter $\Omega^{v}$ ao descarregar o corpo para o estado de tensões $\mathbf{S}^{\infty}$ em um processo infinitamente rápido (no qual $\mathbf{A}^{v}$ é fixo). Tal constatação oferece um contraste ao modelo elasto-plástico, onde a configuração intermediária era assumida livre de tensões.

\subsection{Lei de evolução}

De acordo com Petiteau et al. (2013), Huber e Tsakmakis (2000) e Pascon e Coda (2017), a condição mais simples para que a desigualdade (5.20) seja atendida é a lei de evolução

$$
\mathbf{L}^{v}=\frac{1}{\eta}\left(\mathbf{M}^{e}\right)^{D}
$$

onde $\eta$ é o parâmetro de viscosidade do material. Tal lei pode ser interpretada como uma consequência do princípio da máxima dissipação quando aplicada uma penalização sobre o valor de $\left\|\left(\mathbf{M}^{e}\right)^{D}\right\|$. De fato, nota-se que a equação (5.21) pode ser obtida minimizando-se o funcional

$$
\ell\left(\mathbf{M}^{e}\right)=-d_{i n t}+\frac{1}{2 \eta}\left\|\left(\mathbf{M}^{e}\right)^{D}\right\|^{2}=-\mathbf{M}^{e}: \mathbf{L}^{v}+\frac{1}{2 \eta}\left(\mathbf{M}^{e}\right)^{D}:\left(\mathbf{M}^{e}\right)^{D}
$$

com relação a $\mathbf{M}^{e}$. Como consequência, o valor de $\left\|\left(\mathbf{M}^{e}\right)^{D}\right\|$ será mais próximo de zero quanto maior o coeficiente de penalização $1 / 2 \eta$, isto é, quanto menor o valor de $\eta$.

Aplicando-se (5.5) em (5.21), é possível expressar a lei de evolução na forma:

$$
\dot{\mathbf{A}}^{v}=\frac{1}{\eta}\left(\mathbf{M}^{e}\right)^{D} \mathbf{A}^{v}
$$

ou, ainda, considerando a simetria do tensor de Mandel,

$$
\dot{\mathbf{C}}^{v}=\left(\dot{\mathbf{A}}^{v}\right)^{T} \mathbf{A}^{v}+\left(\mathbf{A}^{v}\right)^{T} \dot{\mathbf{A}}^{v}=\frac{2}{\eta}\left(\mathbf{A}^{v}\right)^{T}\left(\mathbf{M}^{e}\right)^{D} \mathbf{A}^{v} .
$$

A lei de evolução (5.24), utilizada no trabalho de Pascon e Coda (2017), é vantajosa do ponto de vista computacional, pois $\mathbf{C}^{v}$ é um tensor simétrico, ao contrário de $\mathbf{A}^{v}$, necessitando, portanto, de apenas 6 componentes independentes para sua representação. No entanto, sabe-se que partindo-se de $\mathbf{A}^{v}$ é possível calcular o valor de $\mathbf{C}^{v}$, porém, a recíproca não é trivial. Para contornar esse problema, deve-se manipular as expressões da lei constitutiva de forma que essa não dependa de $\mathbf{A}^{v}$. Assim, a lei de evolução (5.24) pode ser reescrita como

$$
\dot{\mathbf{C}}^{v}=\frac{2}{\eta}\left(\mathbf{C S}^{q}\right)^{D} \mathbf{C}^{v}
$$

onde, para a lei neo-Hookeana (5.14), a tensão não-equilibrante é dada por (PASCON; CODA, 2017):

$$
\mathbf{S}^{q}=\Lambda^{e} \ln \left(J^{e}\right) \mathbf{C}^{-1}+\mu^{e}\left(\left(\mathbf{C}^{v}\right)^{-1}-\mathbf{C}^{-1}\right) .
$$

Tal artifício, entretanto, requer uma readaptação para cada lei constitutiva utilizada. Dessa forma, para manter a generalidade das expressões apresentadas, opta-se por utilizar neste trabalho a lei de evolução (5.23). 
Analogamente à propriedade da conservação do volume plástico, apresentada na subseção 4.2.4, pode-se provar que a lei de evolução adotada garante a conservação do jacobiano viscoso $J^{v}=\operatorname{det}\left(\mathbf{A}^{v}\right)$, pois, utilizando a lei de evolução (5.23) e as relações tensoriais (A.23) e (A.35):

$$
\dot{J}^{v}=\frac{d\left(\operatorname{det}\left(\mathbf{A}^{v}\right)\right)}{d \mathbf{A}^{v}}: \dot{\mathbf{A}}^{v}=\frac{1}{\eta} \operatorname{det}\left(\mathbf{A}^{v}\right)\left(\mathbf{A}^{v}\right)^{-T}:\left(\mathbf{M}^{e}\right)^{D} \mathbf{A}^{v}=\frac{J^{v}}{\eta} \operatorname{tr}\left(\left(\mathbf{M}^{e}\right)^{D}\right)=0 .
$$

\subsection{Implementação computacional}

Assim como no caso elasto-plástico, utiliza-se o método implícito de Euler para integração temporal da lei de evolução (5.23), o que resulta em

$$
\Delta \mathbf{A}^{v}=\mathbf{A}_{s+1}^{v}-\mathbf{A}_{s}^{v}=\frac{\Delta t}{\eta}\left(\mathbf{M}^{e}\right)_{s+1}^{D} \mathbf{A}_{s+1}^{v}
$$

onde os índices $(\cdot)_{s+1} \mathrm{e}(\cdot)_{s}$ denotam os valores das variáveis no passo atual e anterior, respectivamente. Dessa forma, o valor de $\mathbf{A}^{v}$ deve ser determinado, para cada passo de tempo, pelo sistema não-linear

$$
\mathbf{R}^{v}=\mathbf{A}^{v}-\mathbf{A}_{s}^{v}-\frac{\Delta t}{\eta}\left(\mathbf{M}^{e}\right)^{D} \mathbf{A}^{v}=\mathbf{0}
$$

onde, para simplificar a notação, os índices $(\cdot)_{s+1}$ foram omitidos.

Devido ao caráter desviador da lei de evolução, o tensor $\mathbf{A}^{v}$ e todos os tensores envolvidos na lei constitutiva devem ser considerados na sua forma tensorial completa $(3 \times 3)$, mesmo em problemas bidimensionais. Dessa forma, o sistema (5.29) consiste em 9 equações, sendo resolvido, neste trabalho, pelo método de Newton-Raphson (ver detalhamento na seção B.2).

\subsection{Operador tangente consistente visco-elástico}

Com base na equação (5.19), o operador tangente consistente do modelo viscoelástico $\mathfrak{C}^{v e}$ pode ser escrito como:

$$
\mathfrak{C}^{v e}=\frac{d \mathbf{S}}{d \mathbf{E}}=\mathfrak{C}^{\infty}+\frac{d\left(\mathbf{A}^{v}\right)^{-1}}{d \mathbf{E}} \mathbf{S}^{e}\left(\mathbf{A}^{v}\right)^{-T}+\left(\mathbf{A}^{v}\right)^{-1} \frac{d \mathbf{S}^{e}}{d \mathbf{E}}\left(\mathbf{A}^{v}\right)^{-T}+\left(\mathbf{A}^{v}\right)^{-1} \mathbf{S}^{e} \frac{d\left(\mathbf{A}^{v}\right)^{-T}}{d \mathbf{E}} .
$$

Para que seja possível calcular as derivadas $d\left(\mathbf{A}^{v}\right)^{-1} / d \mathbf{E}$ e $d \mathbf{S}^{e} / d \mathbf{E}$, é preciso conhecer o valor de $d \mathbf{A}^{v} / d \mathbf{E}$. Partindo do fato que $\mathbf{R}^{v}=\mathbf{0}$ para todos os passos de tempo, pode-se aplicar a aproximação

$$
\Delta \mathbf{R}^{v}=\mathbf{0} \approx \frac{\partial \mathbf{R}^{v}}{\partial \mathbf{E}}: \Delta \mathbf{E}+\frac{\partial \mathbf{R}^{v}}{\partial \mathbf{A}^{v}}: \Delta \mathbf{A}^{v}
$$

de onde, isolando-se $\Delta \mathbf{A}^{v}$ resulta:

$$
\Delta \mathbf{A}^{v} \approx\left(\frac{\partial \mathbf{R}^{v}}{\partial \mathbf{A}^{v}}\right)^{-1}: \frac{\partial \mathbf{R}^{v}}{\partial \mathbf{E}}: \Delta \mathbf{E},
$$

onde o tensor inverso de quarta ordem $\left(\partial \mathbf{R}^{v} / \partial \mathbf{A}^{v}\right)^{-1}$ pode ser calculado conforme a seção A.3. Para intervalos de tempo suficientemente pequenos, a partir de (5.32), pode-se 
considerar

$$
\frac{d \mathbf{A}^{v}}{d \mathbf{E}} \approx\left(\frac{\partial \mathbf{R}^{v}}{\partial \mathbf{A}^{v}}\right)^{-1}: \frac{\partial \mathbf{R}^{v}}{\partial \mathbf{E}} .
$$

Portanto, assim como no caso visco-plástico, o operador tangente consistente viscoelástico calculado neste trabalho é aproximado, pois sua precisão depende da discretização temporal adotada.

\subsection{Exemplos de verificação}

A implementação do modelo visco-elástico do presente trabalho é verificada com base nos resultados de Pascon e Coda (2017) para os exemplos de tensão uniaxial e cisalhamento simples. Os parâmetros do material adotado, mantidos adimensionais, são apresentados na Tabela 6. Nesse, a massa é desprezada, de forma que as análises são consideradas quase-estáticas, apesar de haver dependência temporal no modelo.

Tabela 6 - Parâmetros do material, extraídos de Pascon e Coda (2017)

\begin{tabular}{ccccc}
\hline$\Lambda^{\infty}$ & $\mu^{\infty}$ & $\Lambda^{e}$ & $\mu^{e}$ & $\eta$ \\
\hline 1000 & 10 & 500 & 8 & variável \\
\hline
\end{tabular}

\subsubsection{Chapa sob tensão uniaxial}

Considera-se neste exemplo uma chapa retangular restrita horizontalmente na face esquerda e verticalmente na face inferior, semelhante à Figura 18, porém, com dimensões $1,0 \times 0,5$ e espessura 0,5 . Novamente, adotam-se 2 elementos finitos de ordem linear com 1 ponto de integração em cada, e estado plano de tensões.

Neste exemplo três casos são considerados, sendo os dois primeiros para verificação (subseções 5.6.1.1 e 5.6.1.2), e o último (subseção 5.6.1.3) para caracterizar o modelo visco-elástico quando submetido a diferentes taxas de carregamento.

\subsubsection{Ensaio de fluência}

No primeiro caso, aplica-se força horizontal de valor total $p=20$ na face direita da chapa. Embora no exemplo original o carregamento seja aplicado subitamente, a mudança excessiva de configuração entre passos consecutivos provoca, neste caso, a não-convergência do algoritmo de estado plano de tensões. Portanto, neste trabalho, substitui-se a carga súbita por uma carga crescente no tempo de 0 a $0,1 \mathrm{~s}$, seguida por uma carga constante no tempo de $0,1 \mathrm{~s}$ até $100 \mathrm{~s}$. Foram utilizados 10000 passos de tempo com incrementos de $\Delta t=0,01 \mathrm{~s}$ cada, isto é, 10 passos na etapa crescente da carga, e 9990 para a etapa de carga constante. Os gráficos de alongamento e tensão são apresentados na Figura 32 para diversos valores de viscosidade, mostrando uma boa concordância com o trabalho de Pascon e Coda (2017). 
Figura 32 - Gráficos para o ensaio de fluência em chapa sob tensão uniaxial

(a) Alongamento axial ao longo do tempo

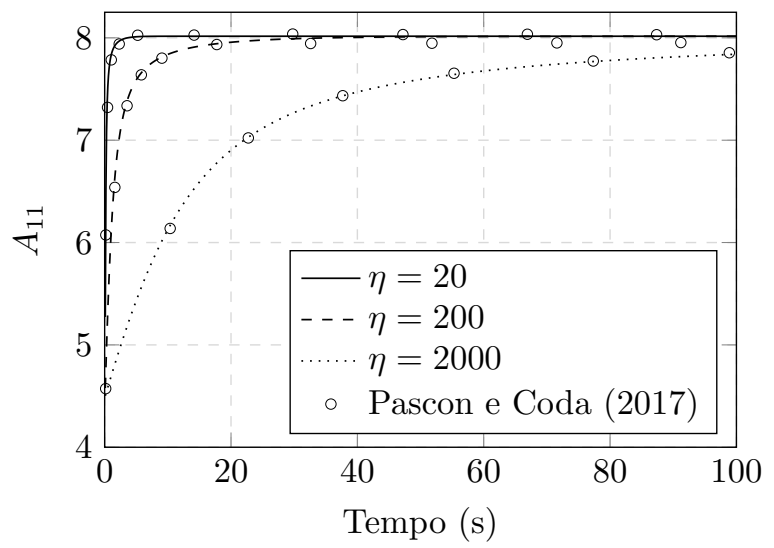

(b) Tensão ao longo do tempo

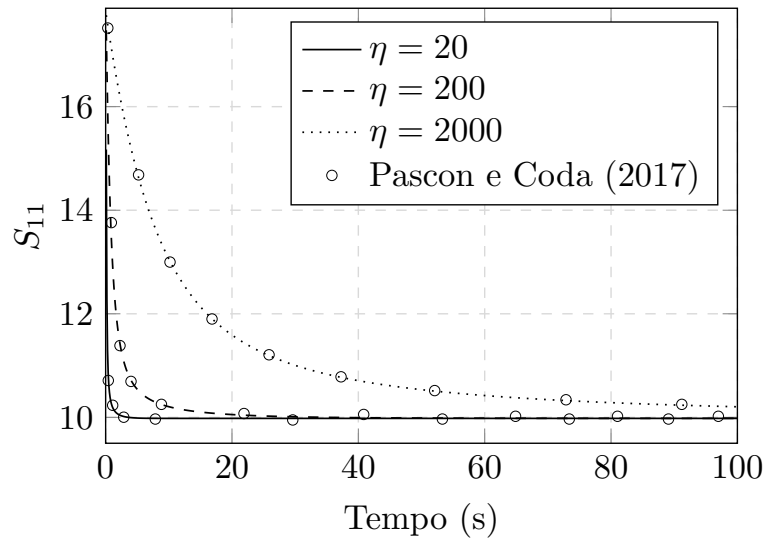

\subsubsection{Ensaio de relaxamento}

No segundo caso, aplica-se deslocamento prescrito $u=4$ na face direita da chapa. Novamente foram adotados 10000 passos de tempo, e o deslocamento é considerado crescente nos tempos de 0 a 0,1 s e constante de 0,1 s até o tempo máximo de análise $t_{\max }=50 \mathrm{~s}$. Os resultados mostram novamente uma boa concordância com a referência, como pode ser visto na Figura 33.

Figura 33 - Gráficos para o ensaio de relaxamento em chapa sob tensão uniaxial

(a) Deformação viscosa ao longo do tempo

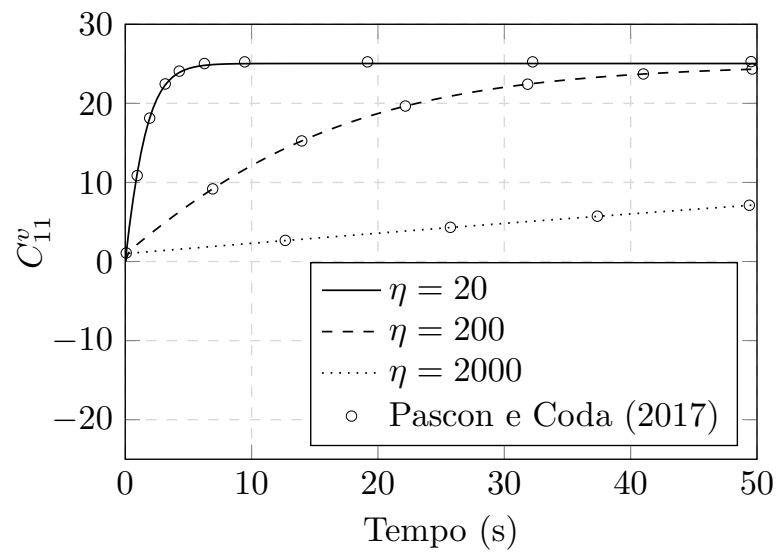

(b) Tensão ao longo do tempo

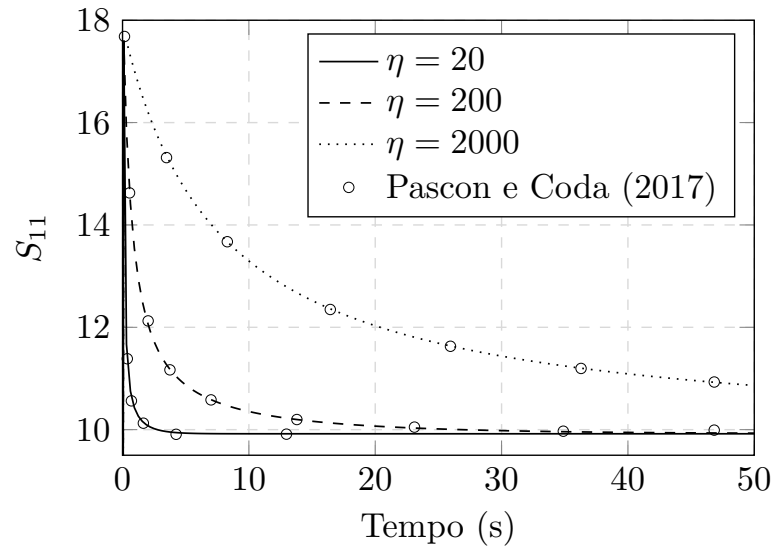

Para ilustrar o comportamento da lei de evolução nos ensaios de fluência e relaxamento, apresenta-se na Figura 34 a evolução das variáveis ao longo do tempo para o caso com $\eta=200$. No ensaio de fluência, observa-se que o alongamento viscoso tende ao alongamento total à medida que o tempo cresce. Consequentemente, o efeito das deformações elásticas $\mathbf{E}^{e}$ é reduzido ao longo do tempo, fazendo com que a resposta se aproxime do modelo hiperelástico I, que leva em conta apenas a lei constitutiva de $\Psi^{\infty}$ (ver Tabela 7 ).

Para o ensaio de relaxamento, observa-se que, quando o tempo tende a infinito, o valor de $\mathbf{S}$ do modelo visco-elástico tende a $\mathbf{S}^{\infty}$, que, naturalmente, coincide com a tensão do modelo hiperelástico I. Como consequência, a tensão não-equilibrante tende a zero. 
Figura 34 - Evolução de variáveis ao longo do tempo para $\eta=200$

(a) Ensaio de fluência

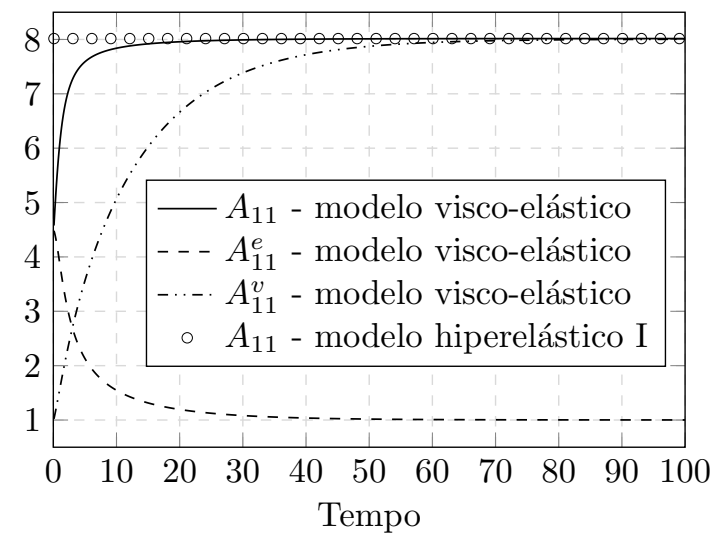

(b) Ensaio de relaxamento

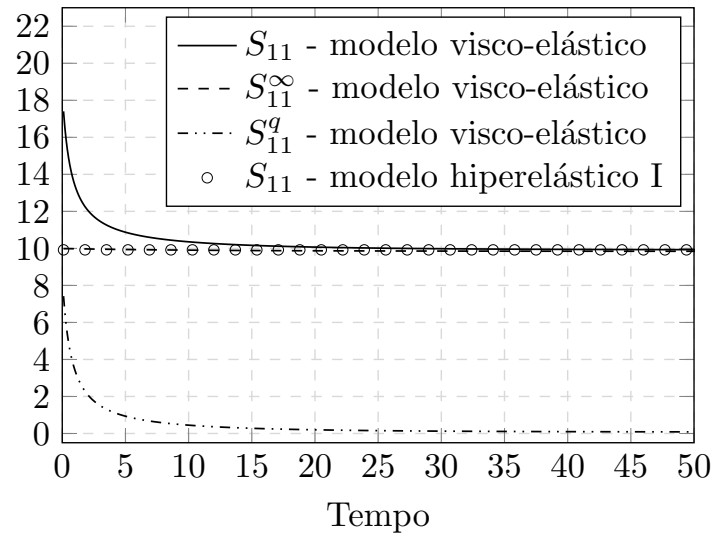

\subsubsection{Carregamento crescente}

A fim de analisar o comportamento dos materiais visco-elásticos quando submetidos a diferentes taxas de deformação, modifica-se o exemplo anterior considerando cargas progressivas ao invés de súbitas. Os resultados obtidos são comparados com os modelos hiperelásticos I, II, e III, definidos conforme a Tabela 7.

Tabela 7 - Leis hiperelásticas associadas ao modelo visco-elástico

\begin{tabular}{cccc}
\hline Modelo & Lei & $\Lambda$ & $\mu$ \\
\hline Hiperelástico I & neo-Hookeana (subseção 2.5.2) & $\Lambda^{\infty}$ & $\mu^{\infty}$ \\
Hiperelástico II & neo-Hookeana (subseção 2.5.2) & $\Lambda^{\infty}+\Lambda^{e}$ & $\mu^{\infty}+\mu^{e}$ \\
Hiperelástico III & neo-Hookeana (subseção 2.5.2) & $\Lambda^{\infty}+\Lambda^{e}$ & $\mu^{\infty}$ \\
\hline
\end{tabular}

Inicialmente, aplica-se uma força total $p=20$ ao longo de 1000 passos com 5 taxas diferentes, sendo variado o tempo máximo de análise. Na menor taxa, $\dot{p}=20 \cdot 10^{-3}$, atinge-se o carregamento máximo no tempo $t_{\max }=10^{3} \mathrm{~s}$. Nesse caso, conforme visto na Figura 35, o alongamento coincide com os dos modelos hiperelásticos I e III. Já a taxa mais alta, $\dot{p}=20 \cdot 10^{2}$, onde o tempo máximo é $t_{\max }=10^{-2} \mathrm{~s}$, coincide com os valores do modelo hiperelástico II. Para as taxas intermediárias, observa-se uma tendência aos modelos I e III quando $t \rightarrow \infty$, e ao hiperelástico II quando $t$ é próximo de 0 .

Tal comportamento é o esperado, uma vez que, para deformações quase instantâneas, o efeito do amortecedor pode ser desprezado, fazendo com que o modelo reológico da Figura 31 se aproxime de uma associação em paralelo das molas $\Psi^{\infty}$ e $\Psi^{e}$ (modelo hiperelástico II). Já para taxas infinitamente baixas (ou quando $t \rightarrow \infty$ ), o efeito do amortecedor provoca uma redução da energia $\Psi^{e}$, o que pode fazer com que a energia livre de Helmholtz tenda a $\Psi^{\infty}$ (modelo hiperelástico I). No entanto, sabe-se que o amortecedor só é capaz de reduzir a parcela não-volumétrica de $\Psi^{e}$. Portanto, um modelo mais ideal para ser associado ao caso de taxas infinitamente lentas é o hiperelástico III, onde consideram-se as parcelas volumétricas de $\Psi^{\infty}$ e $\Psi^{e}$, porém, a parcela não-volumétrica apenas de $\Psi^{\infty}$. 
Figura 35 - Chapa sob tensão uniaxial com carregamento progressivo

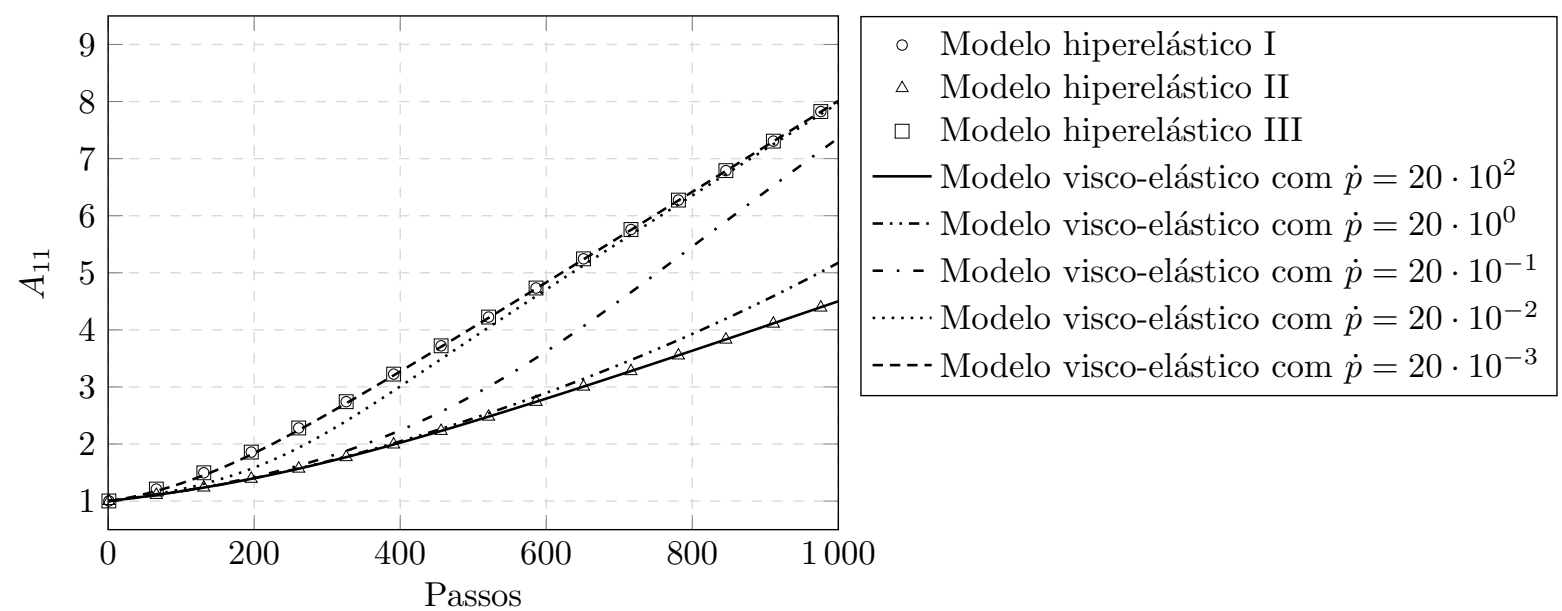

Para ilustrar, modifica-se o exemplo apresentado de forma que hajam diferenças notáveis entre os modelos hiperelásticos I e III. Substitui-se a força total $p=20$ por $p=-20$ (compressiva), e adota-se o material visco-elástico da Tabela 8.

Tabela 8 - Parâmetros alternativos para material visco-elástico

\begin{tabular}{ccccc}
\hline$\Lambda^{\infty}$ & $\mu^{\infty}$ & $\Lambda^{e}$ & $\mu^{e}$ & $\eta$ \\
\hline 320 & 80 & 320 & 100 & 200 \\
\hline
\end{tabular}

Os alongamentos obtidos neste caso são apresentados na Figura 36. Conforme discutido, as respostas para taxas mais baixas tendem aproximadamente ao modelo hiperelástico III, enquanto o hiperelástico I não está associado a nenhuma resposta viscoelástica. Já as taxas quase instantâneas continuam associadas ao modelo hiperelástico II.

Figura 36 - Chapa sob tensão uniaxial compressiva com material visco-elástico II

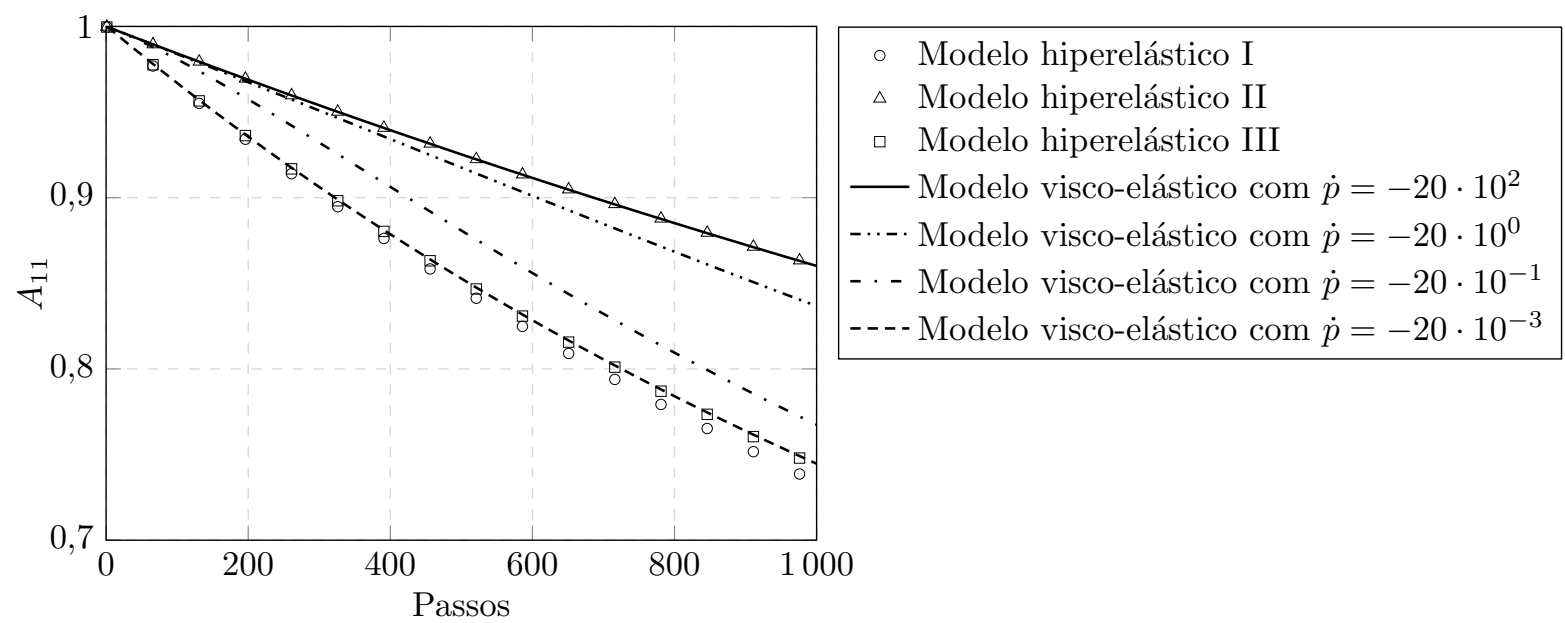




\subsubsection{Chapa sob cisalhamento simples}

Considera-se neste exemplo uma chapa retangular sujeita a deformações cisalhantes, com geometria e malha semelhante à mostrada na Figura 20, porém, com evolução do deslocamento prescrito em forma de pulso triangular, conforme mostrado na Figura 37.

Figura 37 - Gráfico de tempo e deslocamento para o exemplo de cisalhamento simples

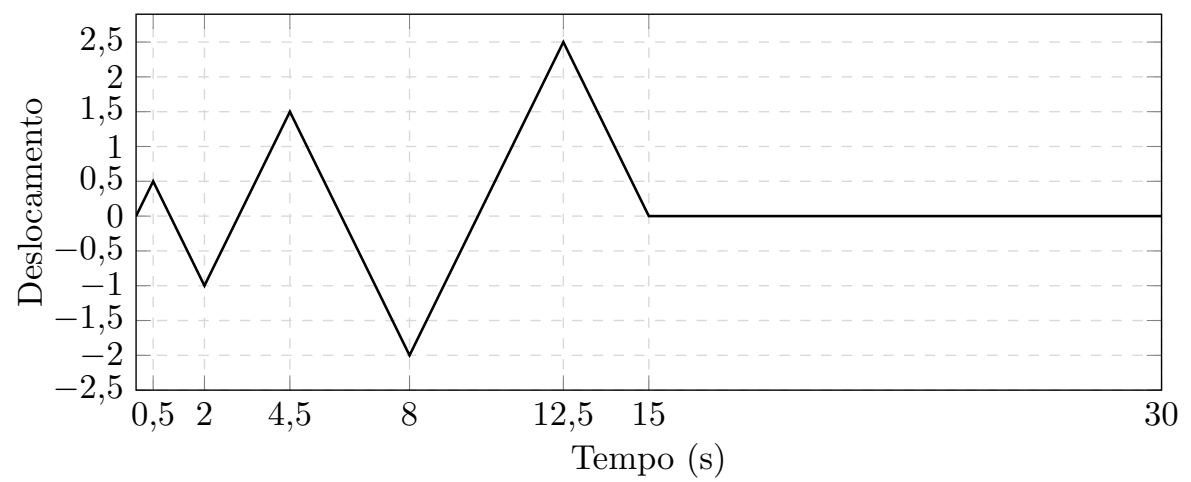

O tempo máximo de análise adotado é 30 s. Como a convergência neste exemplo é imediata, 300 passos de tempo (isto é, $\Delta t=0,1 \mathrm{~s}$ ) mostram-se suficientes para alcançar o resultado desejado. O parâmetro de viscosidade considerado é $\eta=20$. Além disso, foi adotado o estado plano de deformações.

Na Figura 38a apresenta-se o diagrama de tensão-deformação obtido e sua verificação com o trabalho de Pascon e Coda (2017). Pode-se observar, neste exemplo, que as curvas da etapa de carregamento não coincidem com as de descarregamento, o que é denominado efeito de histerese. Observa-se, ainda, que a partir do tempo 15, quando a chapa retorna à sua configuração inicial, a tensão de Cauchy tende a 0 quando $t \rightarrow \infty$, conforme mostrado na Figura 38b.

Figura 38 - Verificações do exemplo de cisalhamento simples em material viscoelástico

(a) Diagrama tensão-deformação

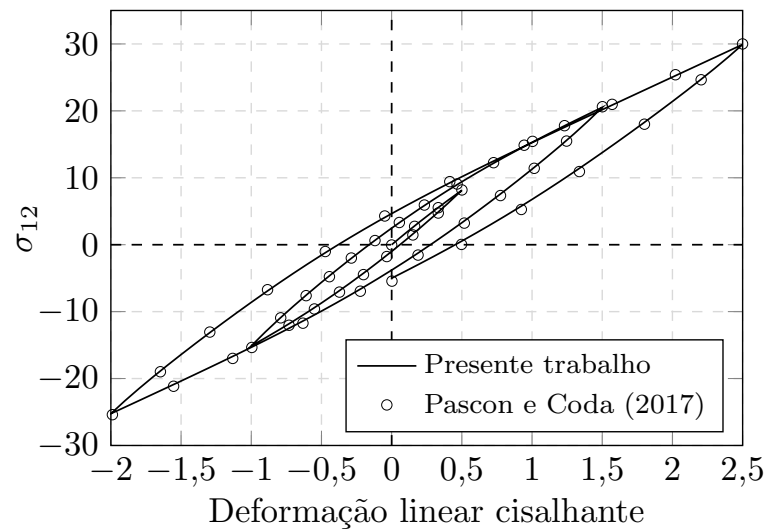

(b) Diagrama tensão-tempo

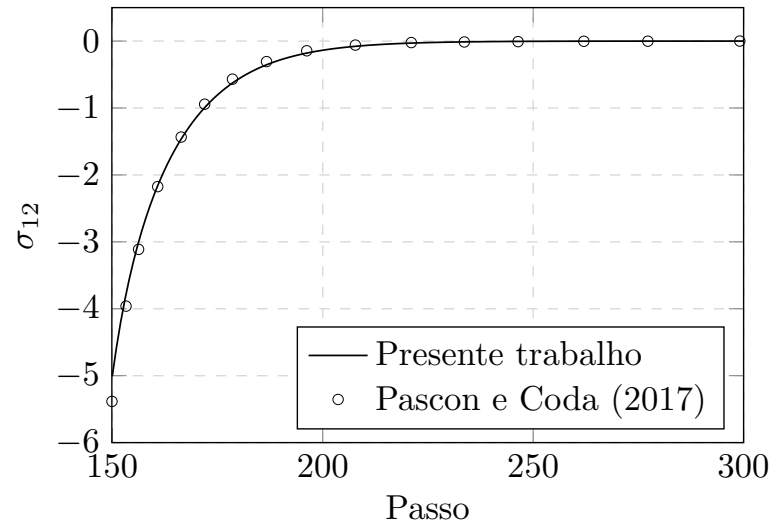




\section{Capítulo 6}

\section{Modelo visco-elasto-plástico}

Um modelo constitutivo dito visco-elasto-plástico (ou viscoelástico-viscoplástico, como é comumente chamado na literatura) caracteriza-se por apresentar dependência temporal tanto nas parcelas elásticas das deformações quanto nas plásticas. Tal comportamento é observado e vem sendo estudado em diversos materiais, como os asfálticos (DRESCHER; KRINGOS; SCARPAS, 2010; DARABI et al., 2011), metálicos em altas temperaturas (BENAARBIA; ROUSE; SUN, 2018) e poliméricos (PRAUD et al., 2017), incluindo materiais empregados em processos de manufatura aditiva (LAMMENS et al., 2017).

Neste capítulo, apresenta-se um modelo constitutivo visco-elasto-plástico baseado na energia de dissipação para sólidos em grandes deformações, incluindo aplicação numérica. Para isso, é realizado um acoplamento entre o modelo visco-plástico descrito no Capítulo 4 e o visco-elástico descrito no Capítulo 5. De forma a evitar repetições desnecessárias, diversos conceitos já estabelecidos nesses capítulos são retomados aqui de forma direta.

\subsection{Cinemática}

A cinemática adotada no presente modelo parte da apresentada na subseção 4.2.1 para o caso elasto-plástico, substituindo as variáveis elásticas $(\cdot)^{e}$ por visco-elásticas $(\cdot)^{v e}$. Dessa forma, a decomposição de Kröner-Lee (4.32), é reformulada como

$$
\mathbf{A}=\mathbf{A}^{v e} \mathbf{A}^{p}
$$

onde $\mathbf{A}^{\text {ve }}$ representa a parcela visco-elástica do gradiente da mudança de configuração, e $\mathbf{A}^{p}$ a parcela plástica. Pela cinemática do modelo visco-elástico, apresentada na seção 5.1, tem-se que

$$
\mathbf{A}^{v e}=\mathbf{A}^{e} \mathbf{A}^{v}
$$

onde $\mathbf{A}^{e}$ representa a parcela elástica do gradiente da mudança de configuração, e $\mathbf{A}^{v}$ a parcela viscosa. Dessa forma, da aplicação de (6.2) em (6.1), resulta a decomposição

$$
\mathbf{A}=\mathbf{A}^{e} \mathbf{A}^{v} \mathbf{A}^{p}
$$


Além disso, $\mathbf{A}^{p}$ pode ser decomposto multiplicativamente em suas parcelas $\mathbf{A}_{e}^{p}$ e $\mathbf{A}_{i}^{p}$, conforme já discutido na subseção 4.2.1. Tal decomposição, aliada à (6.3), induz a definição das configurações intermediárias $\Omega_{i}^{p}$, $\Omega^{p}$ e $\Omega^{v p}$, ilustradas de forma esquemática na Figura 39, com seus respectivos mapeamentos. As configurações $\Omega_{i}^{p}$ e $\Omega^{p}$ já foram apresentadas anteriormente na subseção 4.2.1, porém, neste caso, $\Omega^{p}$ perde o significado físico ao qual foi associada originalmente, uma vez que essa não é obtida no tempo imediato após o descarregamento, mas sim em um tempo infinitamente grande, quando as deformações visco-elásticas se aproximam de zero. Já a configuração $\Omega^{v p}$ representa o estado do sólido em uma situação teórica onde há apenas deformações viscosas e plásticas.

Figura 39 - Esquema de configurações intermediárias do modelo visco-elasto-plástico

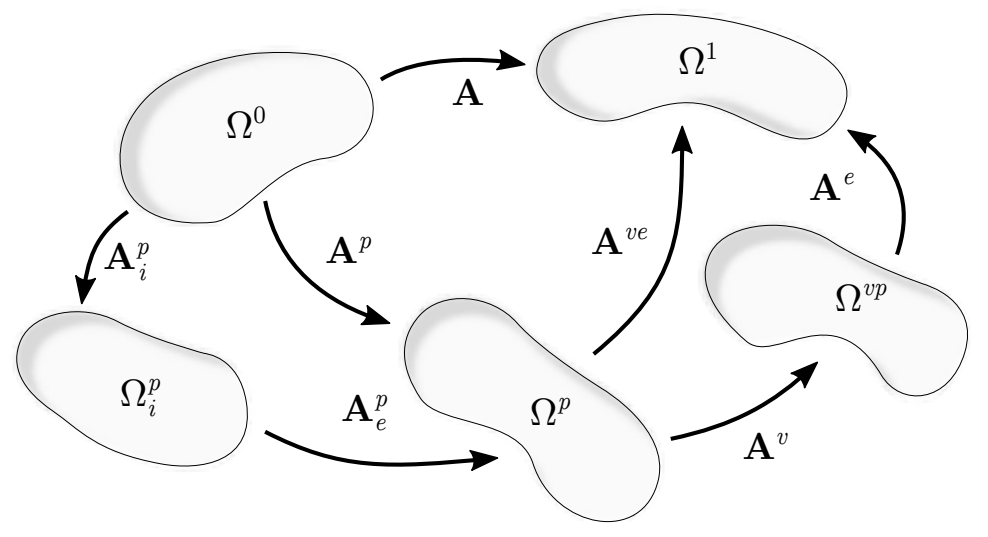

As deformações elásticas, viscosas e plásticas são definidas por expressões análogas às já apresentadas nos modelos elasto-plásticos e visco-elásticos, isto é:

$$
\begin{aligned}
& \mathbf{E}^{e}=\frac{1}{2}\left(\left(\mathbf{A}^{e}\right)^{T} \mathbf{A}^{e}-\mathbf{I}\right)=\frac{1}{2}\left(\mathbf{C}^{e}-\mathbf{I}\right), \\
& \mathbf{E}^{v}=\frac{1}{2}\left(\left(\mathbf{A}^{v}\right)^{T} \mathbf{A}^{v}-\mathbf{I}\right)=\frac{1}{2}\left(\mathbf{C}^{v}-\mathbf{I}\right), \quad \mathrm{e} \\
& \mathbf{E}^{p}=\frac{1}{2}\left(\left(\mathbf{A}^{p}\right)^{T} \mathbf{A}^{p}-\mathbf{I}\right)=\frac{1}{2}\left(\mathbf{C}^{p}-\mathbf{I}\right) .
\end{aligned}
$$

O mesmo vale para os gradientes de velocidade:

$$
\mathbf{L}^{e}=\dot{\mathbf{A}}^{e}\left(\mathbf{A}^{e}\right)^{-1}, \quad \mathbf{L}^{v}=\dot{\mathbf{A}}^{v}\left(\mathbf{A}^{v}\right)^{-1} \quad \text { e } \quad \mathbf{L}^{p}=\dot{\mathbf{A}}^{p}\left(\mathbf{A}^{p}\right)^{-1} .
$$

Da decomposição (6.3), resulta a seguinte relação:

$$
\mathbf{C}=\left(\mathbf{A}^{e} \mathbf{A}^{v} \mathbf{A}^{p}\right)^{T}\left(\mathbf{A}^{e} \mathbf{A}^{v} \mathbf{A}^{p}\right)=\left(\mathbf{A}^{p}\right)^{T}\left(\mathbf{A}^{v}\right)^{T} \mathbf{C}^{e} \mathbf{A}^{v} \mathbf{A}^{p},
$$

ou, rearranjando,

$$
\mathbf{C}^{e}=\left(\mathbf{A}^{v}\right)^{-T}\left(\mathbf{A}^{p}\right)^{-T} \mathbf{C}\left(\mathbf{A}^{p}\right)^{-1}\left(\mathbf{A}^{v}\right)^{-1}
$$

Também de (6.3), pode-se expressar o gradiente da velocidade como

$$
\begin{aligned}
\mathbf{L} & =\dot{\mathbf{A}} \mathbf{A}^{-1}=\left(\dot{\mathbf{A}} \mathbf{A}^{v} \mathbf{A}^{p}+\mathbf{A}^{e} \dot{\mathbf{A}}^{v} \mathbf{A}^{p}+\mathbf{A}^{e} \mathbf{A}^{v} \dot{\mathbf{A}}^{p}\right)\left(\mathbf{A}^{e} \mathbf{A}^{v} \mathbf{A}^{p}\right)^{-1} \\
& =\mathbf{L}^{e}+\mathbf{A}^{e} \mathbf{L}^{v}\left(\mathbf{A}^{e}\right)^{-1}+\mathbf{A}^{e} \mathbf{A}^{v} \mathbf{L}^{p}\left(\mathbf{A}^{v}\right)^{-1}\left(\mathbf{A}^{e}\right)^{-1}
\end{aligned}
$$


e a taxa de deformação,

$$
\mathbf{D}=\operatorname{sim}(\mathbf{L})=\mathbf{D}^{e}+\operatorname{sim}\left(\mathbf{A}^{e} \mathbf{L}^{v}\left(\mathbf{A}^{e}\right)^{-1}+\mathbf{A}^{e} \mathbf{A}^{v} \mathbf{L}^{p}\left(\mathbf{A}^{v}\right)^{-1}\left(\mathbf{A}^{e}\right)^{-1}\right),
$$

o que, aplicado na equação (2.10), resulta em

$$
\begin{aligned}
\dot{\mathbf{E}} & =\mathbf{A}^{T} \mathbf{D} \mathbf{A} \\
& =\left(\mathbf{A}^{e} \mathbf{A}^{v} \mathbf{A}^{p}\right)^{T}\left[\mathbf{D}^{e}+\operatorname{sim}\left(\mathbf{A}^{e} \mathbf{L}^{v}\left(\mathbf{A}^{e}\right)^{-1}+\mathbf{A}^{e} \mathbf{A}^{v} \mathbf{L}^{p}\left(\mathbf{A}^{v}\right)^{-1}\left(\mathbf{A}^{e}\right)^{-1}\right)\right]\left(\mathbf{A}^{e} \mathbf{A}^{v} \mathbf{A}^{p}\right) \\
& =\left(\mathbf{A}^{p}\right)^{T}\left(\mathbf{A}^{v}\right)^{T} \dot{\mathbf{E}}^{e} \mathbf{A}^{v} \mathbf{A}^{p}+\operatorname{sim}\left(\left(\mathbf{A}^{p}\right)^{T}\left(\mathbf{A}^{v}\right)^{T} \mathbf{C}^{e} \mathbf{L}^{v} \mathbf{A}^{v} \mathbf{A}^{p}+\left(\mathbf{A}^{p}\right)^{T}\left(\mathbf{A}^{v}\right)^{T} \mathbf{C}^{e} \mathbf{A}^{v} \mathbf{L}^{p} \mathbf{A}^{p}\right)
\end{aligned}
$$

ou, rearranjando,

$$
\dot{\mathbf{E}}^{e}=\left(\mathbf{A}^{v}\right)^{-T}\left(\mathbf{A}^{p}\right)^{-T} \dot{\mathbf{E}}\left(\mathbf{A}^{p}\right)^{-1}\left(\mathbf{A}^{v}\right)^{-1}-\operatorname{sim}\left(\mathbf{C}^{e} \mathbf{L}^{v}+\mathbf{C}^{e} \mathbf{A}^{v} \mathbf{L}^{p}\left(\mathbf{A}^{v}\right)^{-1}\right) .
$$

Adicionalmente, partindo da definição de $\mathbf{A}^{\text {ve }}$ apresentada na equação (6.2), pode-se definir a deformação visco-elástica:

$$
\mathbf{E}^{v e}=\frac{1}{2}\left(\left(\mathbf{A}^{v e}\right)^{T} \mathbf{A}^{v e}-\mathbf{I}\right)=\frac{1}{2}\left(\mathbf{C}^{v e}-\mathbf{I}\right),
$$

de onde resultam as relações

$$
\begin{gathered}
\mathbf{C}=\left(\mathbf{A}^{v e} \mathbf{A}^{p}\right)^{T}\left(\mathbf{A}^{v e} \mathbf{A}^{p}\right)=\left(\mathbf{A}^{p}\right)^{T} \mathbf{C}^{v e} \mathbf{A}^{p}, \quad \mathrm{e} \\
\mathbf{C}^{e}=\left(\mathbf{A}^{v e}\left(\mathbf{A}^{v}\right)^{-1}\right)^{T}\left(\mathbf{A}^{v e}\left(\mathbf{A}^{v}\right)^{-1}\right)=\left(\mathbf{A}^{v}\right)^{-T} \mathbf{C}^{v e}\left(\mathbf{A}^{v}\right)^{-1},
\end{gathered}
$$

ou, rearranjando,

$$
\mathbf{C}^{v e}=\left(\mathbf{A}^{p}\right)^{-T} \mathbf{C}\left(\mathbf{A}^{p}\right)^{-1}=\left(\mathbf{A}^{v}\right)^{T} \mathbf{C}^{e} \mathbf{A}^{v}
$$

Além disso, seguindo um procedimento análogo à equação (4.49), chega-se a:

$$
\dot{\mathbf{E}}^{v e}=\left(\mathbf{A}^{p}\right)^{-T} \dot{\mathbf{E}}\left(\mathbf{A}^{p}\right)^{-1}-\frac{1}{2} \mathbf{C}^{v e} \mathbf{L}^{p}-\frac{1}{2}\left(\mathbf{L}^{p}\right)^{T} \mathbf{C}^{v e} .
$$

As definições e relações envolvendo os tensores $\mathbf{A}_{i}^{p}$ e $\mathbf{A}_{e}^{p}$ não são diferentes das apresentadas no modelo elasto-plástico e, portanto, podem ser aproveitadas da subseção 4.2.1.

\subsection{Energia, tensão e dissipação}

Baseado nas equações (4.55) e (5.10), escreve-se a energia livre de Helmholtz do modelo visco-elasto-plástico como

$$
\Psi\left(\mathbf{E}^{v e}, \mathbf{E}^{e}, \mathbf{E}_{e}^{p}, \kappa\right)=\Psi^{\infty}\left(\mathbf{E}^{v e}\right)+\Psi^{e}\left(\mathbf{E}^{e}\right)+\Psi_{c i n}^{p}\left(\mathbf{E}_{e}^{p}\right)+\Psi_{i s o}^{p}(\kappa),
$$

onde $\Psi^{\infty}$ e $\Psi^{e}$ estão associadas à parcela visco-elástica do modelo constitutivo, e $\Psi_{\text {cin }}^{p}$ e $\Psi_{i s o}^{p}$ representam o encruamento da parcela visco-plástica. Da equação (6.19), derivam medidas de tensão análogas às apresentadas nos modelos previamente mencionados:

$$
\mathbf{S}^{\infty}=\frac{\partial \Psi^{\infty}}{\partial \mathbf{E}^{v e}}, \quad \mathbf{S}^{e}=\frac{\partial \Psi^{e}}{\partial \mathbf{E}^{e}}, \quad \mathbf{X}=\frac{\partial \Psi_{c i n}^{p}}{\partial \mathbf{E}_{e}^{p}} \quad \text { e } \quad \sigma_{\kappa}=\frac{\partial \Psi_{i s o}^{p}}{\partial \kappa},
$$

e os operadores

$$
\mathfrak{C}^{\infty}=\frac{\partial \mathbf{S}^{\infty}}{\partial \mathbf{E}^{v e}}, \quad \mathfrak{C}^{e}=\frac{\partial \mathbf{S}^{e}}{\partial \mathbf{E}^{e}} \quad \text { e } \quad \mathfrak{C}_{e}^{p}=\frac{\partial \mathbf{X}}{\partial \mathbf{E}_{e}^{p}} .
$$

Para o caso neo-hookeano, as leis atribuídas à $\Psi^{\infty}, \Psi^{e}$ e $\Psi_{\text {cin }}^{p}$ podem ser tomadas 
conforme as equações (5.13), (5.14) e (4.59), respectivamente. Já a tensão de escoamento, $\sigma_{\kappa}$, pode ser tomada conforme as leis dadas na Tabela 1.

A energia de dissipação do modelo parte novamente da segunda lei da termodinâmica, na forma da desigualdade de Clausius-Duhem (4.13), resultando:

$$
d_{i n t}=\mathbf{S}: \dot{\mathbf{E}}-\mathbf{S}^{\infty}: \dot{\mathbf{E}}^{v e}-\mathbf{S}^{e}: \dot{\mathbf{E}}^{e}-\mathbf{X}: \dot{\mathbf{E}}_{e}^{p}-\sigma_{\kappa} \dot{\kappa} \geq 0,
$$

onde $\mathbf{S}$ é a tensão de Piola-Kirchhoff de segunda espécie. Com base na equação (6.13), a parcela $\mathbf{S}^{e}$ : $\dot{\mathbf{E}}^{e}$ pode ser reescrita como

$$
\mathbf{S}^{e}:\left(\left(\mathbf{A}^{v}\right)^{-T}\left(\mathbf{A}^{p}\right)^{-T} \dot{\mathbf{E}}\left(\mathbf{A}^{p}\right)^{-1}\left(\mathbf{A}^{v}\right)^{-1}-\operatorname{sim}\left(\mathbf{C}^{e} \mathbf{L}^{v}+\mathbf{C}^{e} \mathbf{A}^{v} \mathbf{L}^{p}\left(\mathbf{A}^{v}\right)^{-1}\right)\right),
$$

ou ainda, com base nas relações tensoriais (A.33) e (A.34),

$$
\begin{aligned}
\mathbf{S}^{e}: \dot{\mathbf{E}}^{e}= & \left(\left(\mathbf{A}^{p}\right)^{-1}\left(\mathbf{A}^{v}\right)^{-1} \mathbf{S}^{e}\left(\mathbf{A}^{v}\right)^{-T}\left(\mathbf{A}^{p}\right)^{-T}\right): \dot{\mathbf{E}}-\frac{1}{2} \mathbf{C}^{e} \mathbf{S}^{e}: \mathbf{L}^{v}-\frac{1}{2} \mathbf{C}^{e} \mathbf{S}^{e}: \mathbf{L}^{v} \\
& -\frac{1}{2}\left(\left(\mathbf{A}^{v}\right)^{T} \mathbf{C}^{e} \mathbf{S}^{e}\left(\mathbf{A}^{v}\right)^{-T}\right): \mathbf{L}^{p}-\frac{1}{2}\left(\left(\mathbf{A}^{v}\right)^{T} \mathbf{C}^{e} \mathbf{S}^{e}\left(\mathbf{A}^{v}\right)^{-T}\right): \mathbf{L}^{p} \\
= & \left(\left(\mathbf{A}^{p}\right)^{-1}\left(\mathbf{A}^{v}\right)^{-1} \mathbf{S}^{e}\left(\mathbf{A}^{v}\right)^{-T}\left(\mathbf{A}^{p}\right)^{-T}\right): \dot{\mathbf{E}}-\mathbf{M}^{e}: \mathbf{L}^{v}-\mathbf{M}^{q}: \mathbf{L}^{p},
\end{aligned}
$$

onde o tensor de Mandel, $\mathbf{M}^{e}=\mathbf{C}^{e} \mathbf{S}^{e}$, neste caso é definido na configuração intermediária viscosa, e $\mathbf{M}^{q}=\left(\mathbf{A}^{v}\right)^{T} \mathbf{M}^{e}\left(\mathbf{A}^{v}\right)^{-T}$ é um tensor do tipo Mandel definido na configuração intermediária plástica.

Aplicando (6.18) na parcela $\mathbf{S}^{\infty}$ : $\dot{\mathbf{E}}^{\text {ve }}$ e adotando o mesmo procedimento realizado em (4.62), chega-se à seguinte expressão:

$$
\begin{aligned}
\mathbf{S}^{\infty}: \dot{\mathbf{E}}^{v e} & =\mathbf{S}^{\infty}:\left(\left(\mathbf{A}^{p}\right)^{-T} \dot{\mathbf{E}}\left(\mathbf{A}^{p}\right)^{-1}-\frac{1}{2} \mathbf{C}^{v e} \mathbf{L}^{p}-\frac{1}{2}\left(\mathbf{L}^{p}\right)^{T} \mathbf{C}^{v e}\right) \\
& =\left(\left(\mathbf{A}^{p}\right)^{-1} \mathbf{S}^{\infty}\left(\mathbf{A}^{p}\right)^{-T}\right): \dot{\mathbf{E}}-\frac{1}{2}\left(\mathbf{C}^{v e} \mathbf{S}^{\infty}\right): \mathbf{L}^{p}-\frac{1}{2}\left(\mathbf{C}^{v e} \mathbf{S}^{\infty}\right): \mathbf{L}^{p} \\
& =\left(\left(\mathbf{A}^{p}\right)^{-1} \mathbf{S}^{\infty}\left(\mathbf{A}^{p}\right)^{-T}\right): \dot{\mathbf{E}}-\mathbf{M}^{\infty}: \mathbf{L}^{p}
\end{aligned}
$$

onde o tensor do tipo Mandel $\mathbf{M}^{\infty}=\mathbf{C}^{v e} \mathbf{S}^{\infty}$, é também definido na configuração intermediária plástica.

Para a parcela $\mathbf{X}: \dot{\mathbf{E}}_{e}^{p}$, pode-se aproveitar o resultado obtido na equação (4.69):

$$
\mathbf{X}: \dot{\mathbf{E}}_{e}^{p}=\chi: \mathbf{L}^{p}-\mathbf{M}_{e}^{p}: \mathbf{L}_{i}^{p}
$$

onde

$$
\boldsymbol{\chi}=\mathbf{A}_{e}^{p} \mathbf{X}\left(\mathbf{A}_{e}^{p}\right)^{T} \quad \text { e } \quad \mathbf{M}_{e}^{p}=\mathbf{C}_{e}^{p} \mathbf{X} .
$$

Finalmente, substituindo-se (4.62) e (4.69) em (4.60), resulta

$$
\begin{aligned}
d_{i n t}= & \left(\mathbf{S}-\left(\mathbf{A}^{p}\right)^{-1} \mathbf{S}^{\infty}\left(\mathbf{A}^{p}\right)^{-T}-\left(\mathbf{A}^{p}\right)^{-1}\left(\mathbf{A}^{v}\right)^{-1} \mathbf{S}^{e}\left(\mathbf{A}^{v}\right)^{-T}\left(\mathbf{A}^{p}\right)^{-T}\right): \dot{\mathbf{E}} \\
& +\left(\mathbf{M}^{\infty}+\mathbf{M}^{q}-\boldsymbol{\chi}\right): \mathbf{L}^{p}+\mathbf{M}^{e}: \mathbf{L}^{v}+\mathbf{M}_{e}^{p}: \mathbf{L}_{i}^{p}-\sigma_{\kappa} \dot{\kappa} \geq 0 .
\end{aligned}
$$

Para garantir a não-negatividade da energia de dissipação para valores arbitrários de $\dot{\mathbf{E}}$ (HOLZAPFEL, 2000), define-se a tensão de Piola-Kirchhoff de segunda espécie como

$$
\mathbf{S}=\left(\mathbf{A}^{p}\right)^{-1} \mathbf{S}^{\infty}\left(\mathbf{A}^{p}\right)^{-T}+\left(\mathbf{A}^{p}\right)^{-1}\left(\mathbf{A}^{v}\right)^{-1} \mathbf{S}^{e}\left(\mathbf{A}^{v}\right)^{-T}\left(\mathbf{A}^{p}\right)^{-T}=\widetilde{\mathbf{S}}^{\infty}+\widetilde{\mathbf{S}}^{q} .
$$


A energia de dissipação é agora expressa por:

$$
d_{i n t}=\boldsymbol{\Sigma}: \mathbf{L}^{p}+\mathbf{M}^{e}: \mathbf{L}^{v}+\mathbf{M}_{e}^{p}: \mathbf{L}_{i}^{p}-\sigma_{\kappa} \dot{\kappa} \geq 0,
$$

onde

$$
\Sigma=\mathbf{M}^{\infty}+\mathbf{M}^{q}-\chi
$$

Nota-se que o tensor $\boldsymbol{\Sigma}$ é termodinamicamente conjugado à $\mathbf{L}^{p}$, sendo então utilizado para definir o critério de plastificação. É ainda interessante observar que

$$
\mathbf{M}^{q}=\left(\mathbf{A}^{v}\right)^{T} \mathbf{C}^{e} \mathbf{S}^{e}\left(\mathbf{A}^{v}\right)^{-T}=\left(\mathbf{A}^{v}\right)^{T} \mathbf{C}^{e} \mathbf{A}^{v}\left(\mathbf{A}^{v}\right)^{-1} \mathbf{S}^{e}\left(\mathbf{A}^{v}\right)^{-T}=\mathbf{C}^{v e} \mathbf{S}^{q},
$$

$\log \mathrm{O}$

$$
\mathbf{M}^{\infty}+\mathbf{M}^{q}=\mathbf{C}^{v e} \mathbf{S}^{\infty}+\mathbf{C}^{v e} \mathbf{S}^{q}=\mathbf{C}^{v e} \mathbf{S}^{v e}
$$

onde $\mathbf{S}^{v e}$ é a tensão de Piola-Kirchhoff de segunda espécie do modelo visco-elástico, dada conforme a Equação (5.19). Dessa forma, o tensor $\mathbf{M}^{\infty}+\mathbf{M}^{q}$ pode ser visto como uma generalização do tensor de Mandel elástico $\left(\mathbf{M}^{e}\right)$ para o caso visco-elástico.

\subsection{Critério de plastificação e leis de evolução}

Baseado na expressão da energia de dissipação obtida (6.28), e no critério de plastificação da equação (4.74) a função critério de plastificação neste contexto é dada por:

$$
\Phi=\left\|\Sigma^{D}\right\|-\sqrt{\frac{2}{3}} \sigma_{\kappa} \leq 0 .
$$

Seguindo-se procedimentos análogos aos apresentados na subseção 4.2.4 e seção 5.3, são obtidas as seguintes leis de evolução:

$$
\left\{\begin{array}{l}
\dot{\mathbf{A}^{v}}=\frac{1}{\eta}\left(\mathbf{M}^{e}\right)^{D} \mathbf{A}^{v}, \\
\dot{\mathbf{A}}^{p}=\dot{\gamma} \mathbf{N}^{p} \mathbf{A}^{p}, \\
\dot{\mathbf{A}}_{i}^{p}=\dot{\gamma} \mathbf{N}_{i}^{p} \mathbf{A}_{i}^{p}, \\
\dot{\kappa}=\dot{\gamma} \sqrt{\frac{2}{3}},
\end{array}\right.
$$

onde

$$
\mathbf{N}^{p}=\frac{\boldsymbol{\Sigma}^{D}}{\left\|\boldsymbol{\Sigma}^{D}\right\|}
$$

e, para a lei de encruamento cinemático de Armstrong-Frederick,

$$
\mathbf{N}_{i}^{p}=\frac{b}{c}\left(\mathbf{M}_{e}^{p}\right)^{D} .
$$

Caso a parcela plástica seja considerada independente de taxa, o multiplicador plástico $\dot{\gamma}$ deve ser calculado pela condição de complementaridade, conforme discutido na subseção 4.1.7. Caso seja considerado o modelo de Perzyna e lei de Norton conforme apresentado na seção 4.3, tal multiplicador deve ser calculado pela equação (4.112).

Para verificar se as leis de evolução adotadas satisfazem a desigualdade de Clausius- 
Duhem, a equação (6.30) é reescrita levando-se em conta as equações (6.35), resultando:

$$
d_{\text {int }}=\dot{\gamma} \Phi+\dot{\gamma} \frac{b}{c}\left\|\left(\mathbf{M}_{e}^{p}\right)^{D}\right\|^{2}+\frac{1}{\eta}\left\|\left(\mathbf{M}^{e}\right)^{D}\right\|^{2} .
$$

As duas últimas parcelas de (6.38) são claramente positivas. Já a primeira parcela é nula para plasticidade independente de taxa (pela condição de complementaridade) e maior ou igual a zero para o modelo de Perzyna. Logo verifica-se que $d_{\text {int }} \geq 0$, como desejado.

Por argumentos análogos aos da subseção 4.2 .4 e seção 5.3, verifica-se ainda que as leis de evolução garantem a propriedade da conservação do volume inelástico, isto é, os jacobianos plásticos $\left(J^{p}\right)$ e viscosos $\left(J^{v}\right)$ são constantes e unitários ao longo do tempo.

\subsection{Implementação computacional}

A implementação computacional do modelo descrito baseia-se em etapas de previsão visco-elástica e correção plástica, isto é, para cada passo de tempo, assume-se inicialmente que o material é puramente visco-elástico, e verifica-se o critério de plastificação (6.34). Caso esse não seja atendido, deve-se evoluir também as variáveis plásticas.

A integração temporal das leis de evolução é realizada neste trabalho pelo método implícito de Euler (backward Euler). Assim, na etapa de correção plástica, resolve-se o seguinte sistema não-linear:

$$
\left\{\begin{array}{l}
\mathbf{R}^{v}=\mathbf{A}_{s+1}^{v}-\mathbf{A}_{s}^{v}-\frac{\Delta t}{\eta}\left(\mathbf{M}^{e}\right)_{s+1}^{D} \mathbf{A}_{s+1}^{v}=\mathbf{0} \\
\mathbf{R}_{1}=\mathbf{A}_{s+1}^{p}-\mathbf{A}_{s}^{p}-\Delta \gamma_{s+1} \mathbf{N}_{s+1}^{p} \mathbf{A}_{s+1}^{p}=\mathbf{0} \\
r_{2}=\kappa_{s+1}-\kappa_{s}-\Delta \gamma_{s+1}\left(r_{\kappa}\right)=0 \\
\mathbf{R}_{3}=\left(\mathbf{A}_{i}^{p}\right)_{s+1}-\left(\mathbf{A}_{i}^{p}\right)_{s}-\Delta \gamma_{s+1} \mathbf{N}_{i}^{p} \mathbf{A}_{i}^{p}=\mathbf{0} \\
r_{4}=0
\end{array}\right.
$$

onde, caso a parcela plástica seja independente de taxa,

$$
r_{4}=\Phi_{s+1}
$$

e, caso seja adotado o modelo de Perzyna,

$$
r_{4}=\Delta \gamma_{s+1}-\frac{\Delta t}{\eta_{p}}\left\langle\left(\frac{\Phi_{s+1}}{\alpha_{p}}\right)^{m}\right\rangle .
$$

Na Figura 40, apresenta-se o passo-a-passo do algoritmo de previsão e correção adotado. 
Figura 40 - Algoritmo de previsão e correção do modelo visco-elasto-plástico

\section{ALGORITMO DE PREVISÃO E CORREÇÃO \\ PARA O PASSO DE TEMPO $s+1$}

\section{PREVISÃO VISCO-ELÁSTICA:}

a) Assume-se como tentativa inicial para as variáveis plásticas os valores do passo anterior, isto é,

$$
\mathbf{A}_{s+1}^{p}=\mathbf{A}_{s}^{p}, \quad \kappa_{s+1}=\kappa_{s} \quad \text { e } \quad\left(\mathbf{A}_{i}^{p}\right)_{s+1}=\left(\mathbf{A}_{i}^{p}\right)_{s},
$$

ou, caso seja o primeiro passo $(s=0)$ :

$$
\mathbf{A}_{s+1}^{p}=\mathbf{I}, \quad \kappa_{s+1}=0 \quad \text { e } \quad\left(\mathbf{A}_{i}^{p}\right)_{s+1}=\mathbf{I} .
$$

b) Resolve-se $\mathbf{A}_{s+1}^{v}$ pelo sistema não-linear

$$
\mathbf{R}^{v}=\mathbf{A}_{s+1}^{v}-\mathbf{A}_{s}^{v}-\frac{\Delta t}{\eta}\left(\mathbf{M}^{e}\right)_{s+1}^{D} \mathbf{A}_{s+1}^{v}=\mathbf{0}
$$

conforme a seção B.2. Caso seja o primeiro passo, $\mathbf{A}_{s}^{v}=\mathbf{I}$.

c) De $\kappa_{s+1}$, calcula-se $\sigma_{\kappa}$ conforme a Tabela 1. Além disso, calcula-se

$$
\begin{aligned}
& \mathbf{A}^{v e}=\mathbf{A}\left(\mathbf{A}^{p}\right)^{-1} \\
& \mathbf{A}^{e}=\mathbf{A}\left(\mathbf{A}^{p}\right)^{-1}\left(\mathbf{A}^{v}\right)^{-1} \\
& \mathbf{A}_{e}^{p}=\mathbf{A}^{p}\left(\mathbf{A}_{i}^{p}\right)^{-1} \\
& \mathbf{C}^{v e}=\left(\mathbf{A}^{v e}\right)^{T} \mathbf{A}^{v e} \\
& \mathbf{C}^{e}=\left(\mathbf{A}^{e}\right)^{T} \mathbf{A}^{e} \\
& \mathbf{S}^{\infty}=2 \frac{\partial \Psi^{\infty}}{\partial \mathbf{C}^{v e}} \\
& \mathbf{M}^{\infty}=\mathbf{C}^{v e} \mathbf{S}^{\infty} \\
& \begin{aligned}
\mathbf{S}^{e} & =2 \frac{\partial \Psi^{e}}{\partial \mathbf{C}^{e}} \\
\mathbf{M}^{q} & =\left(\mathbf{A}^{v}\right)^{T} \mathbf{C}^{e} \mathbf{S}^{e}\left(\mathbf{A}^{v}\right)^{-T}
\end{aligned} \mid \\
& \mathbf{C}_{e}^{p}=\left(\mathbf{A}_{e}^{p}\right)^{T} \mathbf{A}_{e}^{p} \\
& \mathbf{X}=2 \frac{\partial \Psi_{c i n}^{p}}{\partial \mathbf{C}_{e}^{p}} \\
& \chi=\left(\mathbf{A}_{e}^{p}\right) \mathbf{X}\left(\mathbf{A}_{e}^{p}\right)^{T}
\end{aligned}
$$

onde os índices $(\cdot)_{s+1}$ foram omitidos a fim de reduzir a notação.

d) Calcula-se o critério de plastificação

$$
\Phi=\left\|\left(\mathbf{M}^{\infty}+\mathbf{M}^{q}-\chi\right)^{D}\right\|-\sqrt{2 / 3} \sigma_{\kappa} .
$$

2. SE $\Phi \leq 0 \rightarrow$ PREVISÃO VISCO-ELÁSTICA CORRETA:

a) Não há necessidade de atualizar as variáveis previamente calculadas.

b) Calcula-se $\mathfrak{C}^{v e p}$ pela forma simplificada exibida na subseção C.3.1.

3. SE $\Phi>0 \rightarrow$ CORREÇÃO PLÁSTICA:

a) Soluciona-se o sistema (6.39) conforme o Apêndice B, de onde obtêm-se as variáveis atualizadas $\mathbf{A}_{s+1}^{v}, \mathbf{A}_{s+1}^{p}, \kappa_{s+1},\left(\mathbf{A}_{i}^{p}\right)_{s+1}$ e $\Delta \gamma_{s+1}$. Com exceção de $\Delta \gamma_{s+1}$, todas essas variáveis precisam ser armazenadas para serem utilizadas no próximo passo.

b) Atualiza-se $\mathbf{S}_{s+1}^{\infty}, \mathbf{S}_{s+1}^{e}$ e as demais variáveis utilizadas para calcular $\mathfrak{C}^{v e p}$.

c) Calcula-se $\mathfrak{C}^{\text {vep }}$ conforme a subseção C.3.2.

4. Finalmente, calcula-se o tensor de tensões de Piola-Kirchhoff de segunda espécie:

$$
\mathbf{S}=\left(\mathbf{A}^{p}\right)^{-1} \mathbf{S}^{\infty}\left(\mathbf{A}^{p}\right)^{-T}+\left(\mathbf{A}^{p}\right)^{-1}\left(\mathbf{A}^{v}\right)^{-1} \mathbf{S}^{e}\left(\mathbf{A}^{v}\right)^{-T}\left(\mathbf{A}^{p}\right)^{-T}
$$




\subsection{Operador tangente consistente visco-elasto-plástico}

O operador tangente consistente do modelo visco-elasto-plástico pode ser derivado de (6.29) como:

$$
\begin{aligned}
\mathfrak{C}^{v e p}= & \frac{d \mathbf{S}}{d \mathbf{E}}=\frac{d\left(\mathbf{A}^{p}\right)^{-1}}{d \mathbf{E}} \mathbf{S}^{\infty}\left(\mathbf{A}^{p}\right)^{-T}+\left(\mathbf{A}^{p}\right)^{-1} \frac{d \mathbf{S}^{\infty}}{d \mathbf{E}}\left(\mathbf{A}^{p}\right)^{-T}+\left(\mathbf{A}^{p}\right)^{-1} \mathbf{S}^{\infty} \frac{d\left(\mathbf{A}^{p}\right)^{-T}}{d \mathbf{E}} \\
& +\frac{d\left(\mathbf{A}^{p}\right)^{-1}}{d \mathbf{E}}\left(\mathbf{A}^{v}\right)^{-1} \mathbf{S}^{e}\left(\mathbf{A}^{v}\right)^{-T}\left(\mathbf{A}^{p}\right)^{-T}+\left(\mathbf{A}^{p}\right)^{-1}\left(\mathbf{A}^{v}\right)^{-1} \mathbf{S}^{e}\left(\mathbf{A}^{v}\right)^{-T} \frac{d\left(\mathbf{A}^{p}\right)^{-T}}{d \mathbf{E}} \\
& +\left(\mathbf{A}^{p}\right)^{-1} \frac{d\left(\mathbf{A}^{v}\right)^{-1}}{d \mathbf{E}} \mathbf{S}^{e}\left(\mathbf{A}^{v}\right)^{-T}\left(\mathbf{A}^{p}\right)^{-T}+\left(\mathbf{A}^{p}\right)^{-1}\left(\mathbf{A}^{v}\right)^{-1} \mathbf{S}^{e} \frac{d\left(\mathbf{A}^{v}\right)^{-T}}{d \mathbf{E}}\left(\mathbf{A}^{p}\right)^{-T} \\
& +\left(\mathbf{A}^{p}\right)^{-1}\left(\mathbf{A}^{v}\right)^{-1} \frac{d \mathbf{S}^{e}}{d \mathbf{E}}\left(\mathbf{A}^{v}\right)^{-T}\left(\mathbf{A}^{p}\right)^{-T} .
\end{aligned}
$$

Para que possam ser calculadas as derivadas presentes em (6.42), deve-se conhecer os valores de $d \mathbf{A}^{p} / d \mathbf{E}$ e $d \mathbf{A}^{v} / d \mathbf{E}$. Parte-se, neste trabalho, das aproximações

$$
\begin{gathered}
\Delta \mathbf{R}^{v}=\mathbf{0} \approx \frac{\partial \mathbf{R}^{v}}{\partial \mathbf{E}}: \Delta \mathbf{E}+\frac{\partial \mathbf{R}^{v}}{\partial \mathbf{A}^{v}}: \Delta \mathbf{A}^{v}+\frac{\partial \mathbf{R}^{v}}{\partial \mathbf{A}^{p}}: \Delta \mathbf{A}^{p}, \quad \text { e } \\
\Delta r_{4}=0 \approx \frac{\partial r_{4}}{\partial \mathbf{E}}: \Delta \mathbf{E}+\frac{\partial r_{4}}{\partial \mathbf{A}^{p}}: \Delta \mathbf{A}^{p}+\frac{\partial r_{4}}{\partial \kappa} \Delta \kappa+\frac{\partial r_{4}}{\partial \mathbf{A}_{i}^{p}}: \Delta \mathbf{A}_{i}^{p}+\frac{\partial r_{4}}{\partial \mathbf{A}^{v}}: \Delta \mathbf{A}^{v}+\frac{\partial r_{4}}{\partial \gamma} \Delta \gamma .
\end{gathered}
$$

De (6.43), tem-se

$$
\Delta \mathbf{A}^{v} \approx-\left(\frac{\partial \mathbf{R}^{v}}{\partial \mathbf{A}^{v}}\right)^{-1}:\left(\frac{\partial \mathbf{R}^{v}}{\partial \mathbf{E}}: \Delta \mathbf{E}+\frac{\partial \mathbf{R}^{v}}{\partial \mathbf{A}^{p}}: \Delta \mathbf{A}^{p}\right) .
$$

que, aplicada em (6.44), resulta em

$$
\begin{aligned}
\Delta r_{4}=0 \approx & \left(\frac{\partial r_{4}}{\partial \mathbf{E}}-\frac{\partial r_{4}}{\partial \mathbf{A}^{v}}:\left(\frac{\partial \mathbf{R}^{v}}{\partial \mathbf{A}^{v}}\right)^{-1}: \frac{\partial \mathbf{R}^{v}}{\partial \mathbf{E}}\right): \Delta \mathbf{E} \\
& +\left(\frac{\partial r_{4}}{\partial \mathbf{A}^{p}}-\frac{\partial r_{4}}{\partial \mathbf{A}^{v}}:\left(\frac{\partial \mathbf{R}^{v}}{\partial \mathbf{A}^{v}}\right)^{-1}: \frac{\partial \mathbf{R}^{v}}{\partial \mathbf{A}^{p}}\right): \Delta \mathbf{A}^{p} \\
& +\frac{\partial r_{4}}{\partial \kappa} \Delta \kappa+\frac{\partial r_{4}}{\partial \mathbf{A}_{i}^{p}}: \Delta \mathbf{A}_{i}^{p}+\frac{\partial r_{4}}{\partial \gamma} \Delta \gamma
\end{aligned}
$$

de onde, à partir das leis de evolução discretizadas (6.39), escreve-se:

$$
\begin{aligned}
\Delta r_{4}=0 \approx & \left(\frac{\partial r_{4}}{\partial \mathbf{E}}-\frac{\partial r_{4}}{\partial \mathbf{A}^{v}}:\left(\frac{\partial \mathbf{R}^{v}}{\partial \mathbf{A}^{v}}\right)^{-1}: \frac{\partial \mathbf{R}^{v}}{\partial \mathbf{E}}\right): \Delta \mathbf{E} \\
& +\Delta \gamma\left(\frac{\partial r_{4}}{\partial \mathbf{A}^{p}}-\frac{\partial r_{4}}{\partial \mathbf{A}^{v}}:\left(\frac{\partial \mathbf{R}^{v}}{\partial \mathbf{A}^{v}}\right)^{-1}: \frac{\partial \mathbf{R}^{v}}{\partial \mathbf{A}^{p}}\right):\left(\mathbf{N}^{p} \mathbf{A}^{p}\right) \\
& +\Delta \gamma\left(\frac{\partial r_{4}}{\partial \kappa} \sqrt{\frac{2}{3}}+\frac{\partial r_{4}}{\partial \mathbf{A}_{i}^{p}}:\left(\mathbf{N}_{i}^{p} \mathbf{A}_{i}^{p}\right)+\frac{\partial r_{4}}{\partial \gamma}\right)
\end{aligned}
$$

Logo,

$$
\Delta \gamma \approx \mathbf{D}^{\gamma}: \Delta \mathbf{E}
$$


onde

$$
\mathbf{D}^{\gamma}=\frac{\frac{\partial r_{4}}{\partial \mathbf{A}^{v}}:\left(\frac{\partial \mathbf{R}^{v}}{\partial \mathbf{A}^{v}}\right)^{-1}: \frac{\partial \mathbf{R}^{v}}{\partial \mathbf{E}}-\frac{\partial r_{4}}{\partial \mathbf{E}}}{\left(\frac{\partial r_{4}}{\partial \mathbf{A}^{p}}-\frac{\partial r_{4}}{\partial \mathbf{A}^{v}}:\left(\frac{\partial \mathbf{R}^{v}}{\partial \mathbf{A}^{v}}\right)^{-1}: \frac{\partial \mathbf{R}^{v}}{\partial \mathbf{A}^{p}}\right):\left(\mathbf{N}^{p} \mathbf{A}^{p}\right)+\frac{\partial r_{4}}{\partial \kappa} \sqrt{\frac{2}{3}}+\frac{\partial r_{4}}{\partial \mathbf{A}_{i}^{p}}:\left(\mathbf{N}_{i}^{p} \mathbf{A}_{i}^{p}\right)+\frac{\partial r_{4}}{\partial \gamma}} .
$$

Se a plasticidade for considerada independente de taxa, $r_{4}=\Phi, \log 0, \partial r_{4} / \partial \gamma=0$. Caso seja utilizado o modelo de Perzyna, $\partial r_{4} / \partial \gamma=1$, e as demais derivadas de $r_{4}$ podem ser calculadas conforme a equação (4.121) para a lei de Norton.

Da substituição da equação (6.48) na lei de evolução (4.86), escreve-se

$$
\Delta \mathbf{A}^{p}=\Delta \gamma \mathbf{N}^{p} \mathbf{A}^{p} \approx\left(\mathbf{D}^{\gamma}: \Delta \mathbf{E}\right) \mathbf{N}^{p} \mathbf{A}^{p}=\left[\left(\mathbf{N}^{p} \mathbf{A}^{p}\right) \otimes \mathbf{D}^{\gamma}\right]: \Delta \mathbf{E},
$$

que, aplicado em (6.45), resulta

$$
\Delta \mathbf{A}^{v} \approx-\left(\frac{\partial \mathbf{R}^{v}}{\partial \mathbf{A}^{v}}\right)^{-1}:\left(\frac{\partial \mathbf{R}^{v}}{\partial \mathbf{E}}+\frac{\partial \mathbf{R}^{v}}{\partial \mathbf{A}^{p}}:\left(\left(\mathbf{N}^{p} \mathbf{A}^{p}\right) \otimes \mathbf{D}^{\gamma}\right)\right): \Delta \mathbf{E} .
$$

Logo, para passos de tempo suficientemente pequenos,

$$
\begin{gathered}
\frac{d \mathbf{A}^{p}}{d \mathbf{E}} \approx\left(\mathbf{N}^{p} \mathbf{A}^{p}\right) \otimes \mathbf{D}^{\gamma}, \quad \mathrm{e} \\
\frac{d \mathbf{A}^{v}}{d \mathbf{E}} \approx-\left(\frac{\partial \mathbf{R}^{v}}{\partial \mathbf{A}^{v}}\right)^{-1}:\left(\frac{\partial \mathbf{R}^{v}}{\partial \mathbf{E}}+\frac{\partial \mathbf{R}^{v}}{\partial \mathbf{A}^{p}}:\left(\left(\mathbf{N}^{p} \mathbf{A}^{p}\right) \otimes \mathbf{D}^{\gamma}\right)\right) .
\end{gathered}
$$

O cálculo completo de $\mathfrak{C}^{v e p}$ e suas derivadas auxiliares é detalhado na seção C.3.

\subsection{Exemplos numéricos}

Para demonstrar o comportamento do modelo visco-elasto-plástico descrito, nesta seção aplica-se o modelo numérico desenvolvido aos exemplos clássicos de cisalhamento simples e tensão uniaxial, sendo este último considerado no contexto de ensaios de fluência e relaxamento. A fim de simplificar o escopo das análises realizadas, todos os exemplos são considerados quase-estáticos, apesar de haver dependência temporal nos modelos constitutivos. Os parâmetros do material visco-elasto-plástico utilizado são apresentados na Tabela 9, inspirados no material polimérico (PET orientado) da Tabela 2.

Tabela 9 - Parâmetros para material visco-elasto-plástico do tipo polimérico

\begin{tabular}{cccccc}
\hline \multicolumn{5}{c}{ Parâmetros visco-elásticos } \\
\hline$\Lambda^{\infty}(\mathrm{MPa})$ & $\mu^{\infty}(\mathrm{MPa})$ & $\Lambda^{e}(\mathrm{MPa})$ & $\mu^{e}(\mathrm{MPa})$ & $\eta(\mathrm{MPa} \cdot \mathrm{s})$ \\
320 & 80 & 0 & 40 & 50 \\
\hline \multicolumn{2}{c|}{ Parâmetros de encruamento } & \multicolumn{2}{c}{ Parâmetros de Perzyna/Norton } \\
\hline$\sigma_{Y}(\mathrm{MPa})$ & $c(\mathrm{MPa})$ & $b$ & $\eta_{p}(\mathrm{~s})$ & $\alpha_{p}(\mathrm{MPa})$ & $m$ \\
35 & 100 & 2.7 & 1 & 35 & 1 \\
\hline
\end{tabular}




\subsubsection{Ensaio de relaxamento}

Considera-se, neste caso, uma chapa sob tensão uniaxial, com geometria, restrições e malha semelhantes à Figura 18, porém com deslocamento prescrito aplicado conforme o gráfico da Figura 41, sendo o instante final da análise $t_{2}=8 \mathrm{~s}$, e o instante intermediário $t_{1}$ variável. O número de passos adotado é 2000, sendo 1000 para o intervalo de tempo entre 0 e $t_{1}$, e 1000 para o intervalo entre $t_{1}$ e $t_{2}$. Como $t_{1}$ é variável, segue que o incremento de tempo $(\Delta t)$ adotado é diferente nos dois intervalos.

Figura 41 - Evolução do deslocamento prescrito aplicado em ensaio de relaxamento para chapa com modelo visco-elasto-plástico

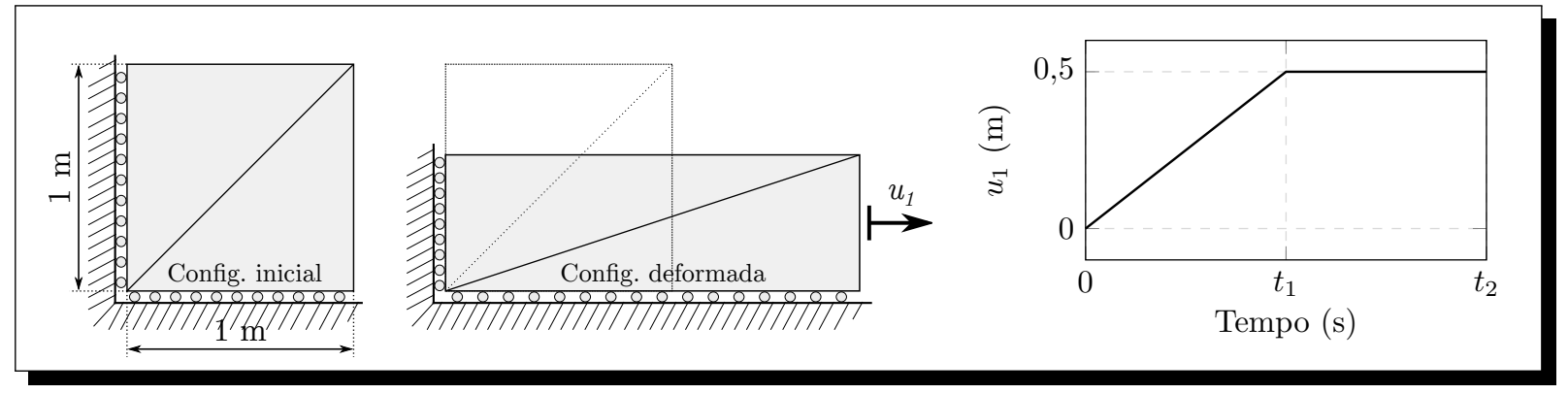

Os valores de tensão de Cauchy na direção longitudinal são dados na Figura 42a para diversas taxas de deslocamento, isto é, diversos valores de $t_{1}$. Observa-se que nas taxas mais altas a tensão obtida no tempo $t_{1}$ é maior, de forma semelhante ao modelo visco-plástico. Entretanto, quando as deformações se estabilizam, a tensão tende a valores menores (em contrate ao modelo visco-plástico, onde as tensões tendem ao mesmo valor). Na Figura 42b, apresenta-se a evolução dos alongamentos plásticos ao longo do tempo para os diversos casos analisados. Na Figura 43 apresenta-se, ainda, uma comparação entre os alongamentos plásticos, viscosos e visco-elásticos para os casos em que $t_{1}=2 \mathrm{~s}$ e $t_{1}=0.1 \mathrm{~s}$.

Figura 42 - Tensões de Cauchy e alongamentos plásticos para ensaio de relaxamento em chapa com material visco-elasto-plástico

(a) Tensão de Cauchy axial

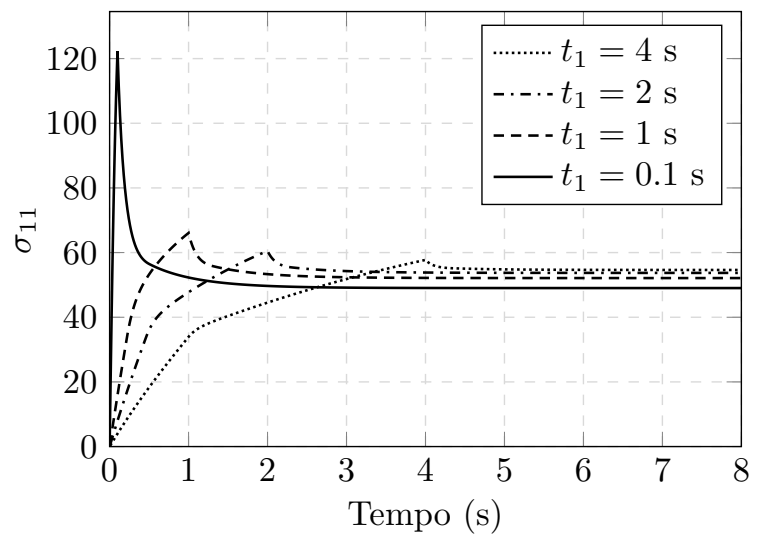

(b) Alongamento plástico

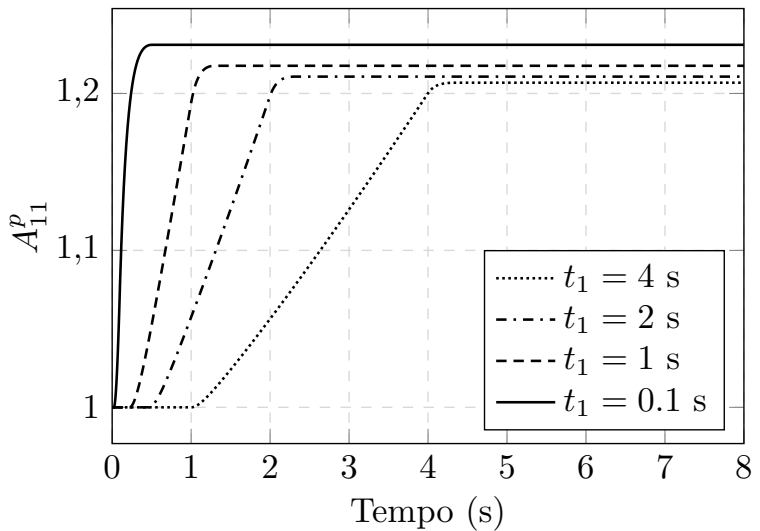


Figura 43 - Alongamentos para ensaio de relaxamento em chapa com material visco-elastoplástico

(a) $t_{1}=2 \mathrm{~s}$

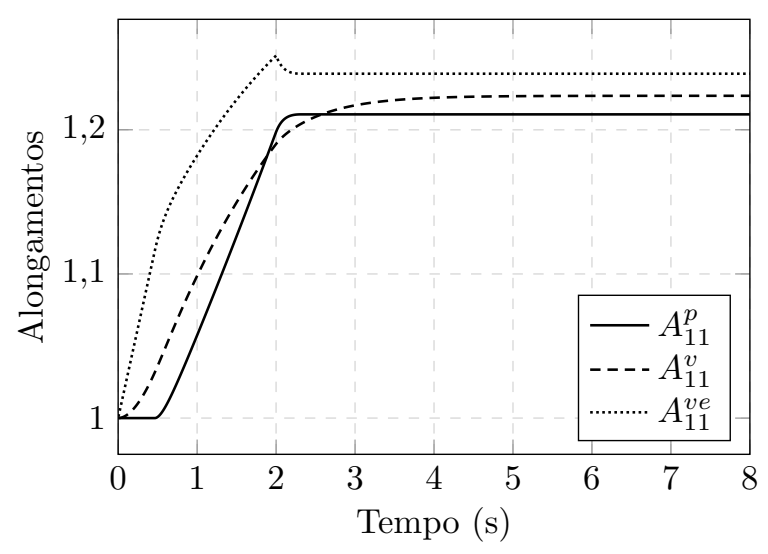

(b) $t_{1}=0.1 \mathrm{~s}$

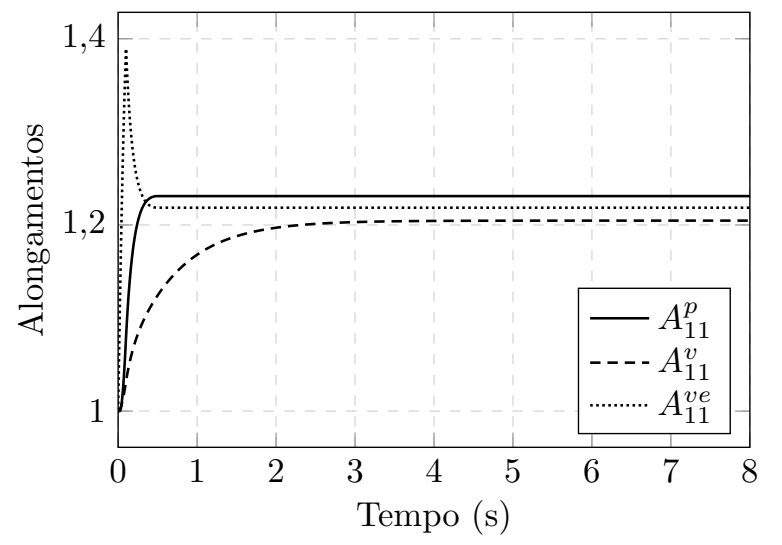

\subsubsection{Ensaio de fluência}

Considera-se, neste caso, uma chapa com geometria e malha semelhantes à do exemplo anterior, porém substituindo o deslocamento prescrito por força compressiva, conforme a Figura 44. Ao contrário do exemplo da subseção 5.6.1.1, este não apresentou problemas de convergência devido às cargas súbitas, mesmo sendo considerado o estado plano de tensões. O instante máximo considerado foi $t_{2}=10 \mathrm{~s}$, discretizado em 1000 passos com $\Delta t=0.01$ s. Já o instante intermediário $\left(t_{1}\right)$ é variado ao longo das análises.

Figura 44 - Evolução da força aplicada em ensaio de fluência para modelo visco-elasto-plástico

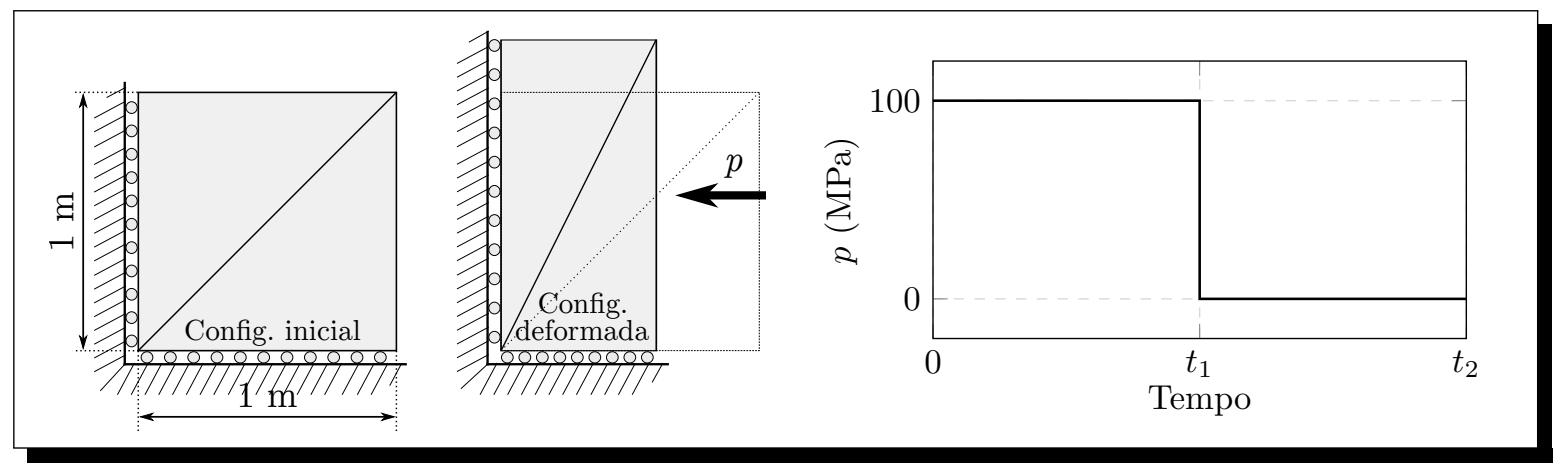

Na Figura 45 são apresentados os deslocamentos horizontais e verticais no vértice superior direito da chapa ao longo do tempo. As respostas apresentam o comportamento esperado em testes de fluência de modelos visco-elasto-plásticos, como pode ser visto, por exemplo, no trabalho de Darabi et al. (2011): para maiores tempos de manutenção da carga, maiores são os deslocamentos residuais obtidos ao se remover o carregamento.

Na Figura 46, apresenta-se a evolução dos alongamentos plásticos e viscosos para os diversos tempos de manutenção do carregamento analisados. Como esperado, o alongamento plástico evolui de forma acentuada no começo do carregamento, porém, tende a se estabilizar com o tempo. Após a remoção da carga, o alongamento plástico é inalterado. Já o alongamento viscoso tende ao valor unitário quando $t \rightarrow \infty$. 
Figura 45 - Deslocamentos no vértice superior direito da chapa para ensaio de fluência

(a) Deslocamento horizontal

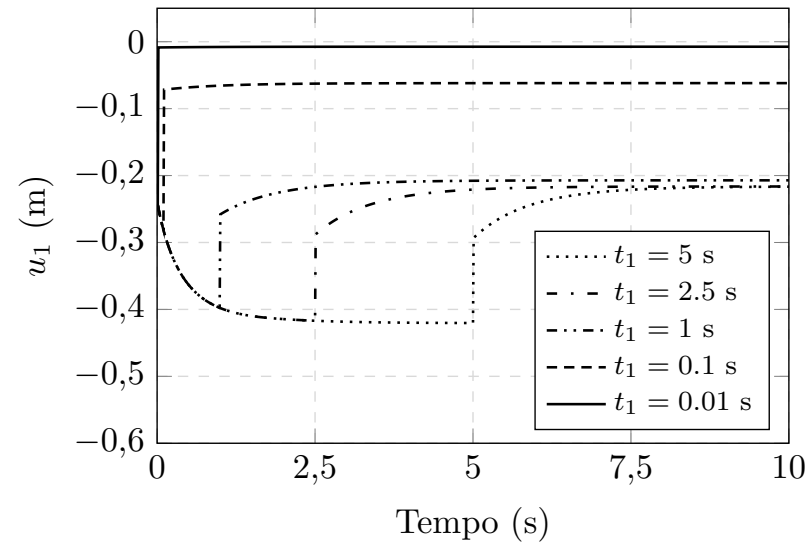

(b) Deslocamento vertical

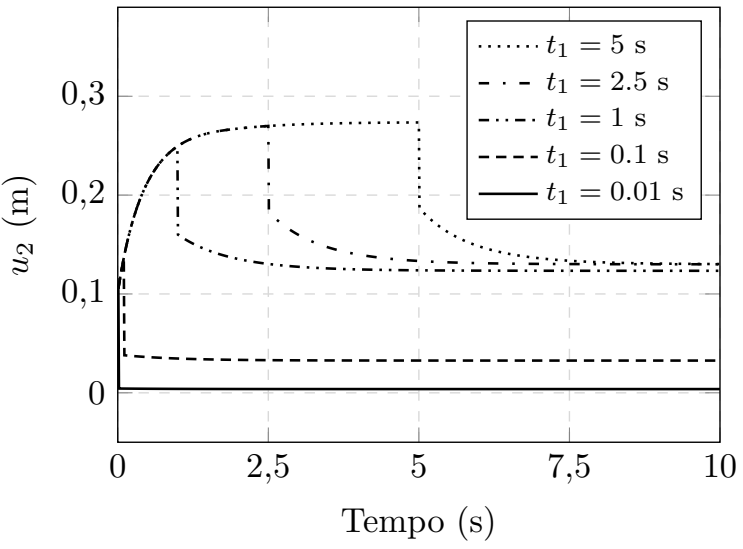

Figura 46 - Alongamentos plásticos e viscosos da chapa para ensaio de fluência

(a) Alongamentos plásticos

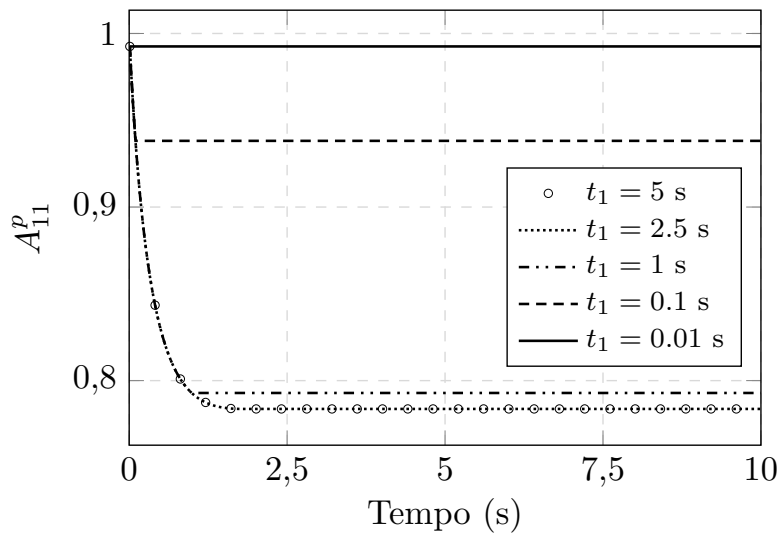

(b) Alongamentos viscosos

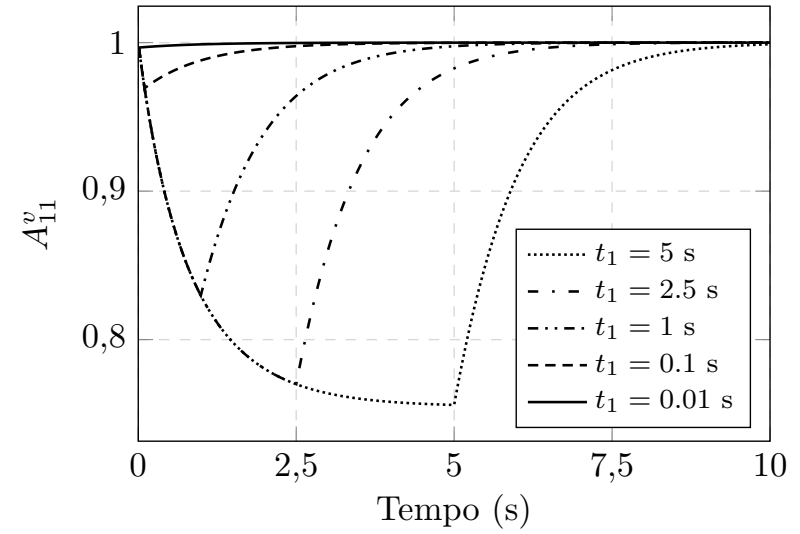

Para facilitar a comparação, apresenta-se na Figura 47a os alongamentos totais e suas parcelas plásticas, viscosas e visco-elásticas para o caso com $t_{1}=5 \mathrm{~s}$. Observa-se que o alongamento visco-elástico sofre uma mudança súbita no descarregamento, devido à sua parcela elástica. Assim como o alongamento viscoso, esse tende ao valor unitário à medida

Figura 47 - Evolução dos alongamentos e tensões para ensaio de fluência com $t_{1}=5 \mathrm{~s}$

(a) Alongamentos

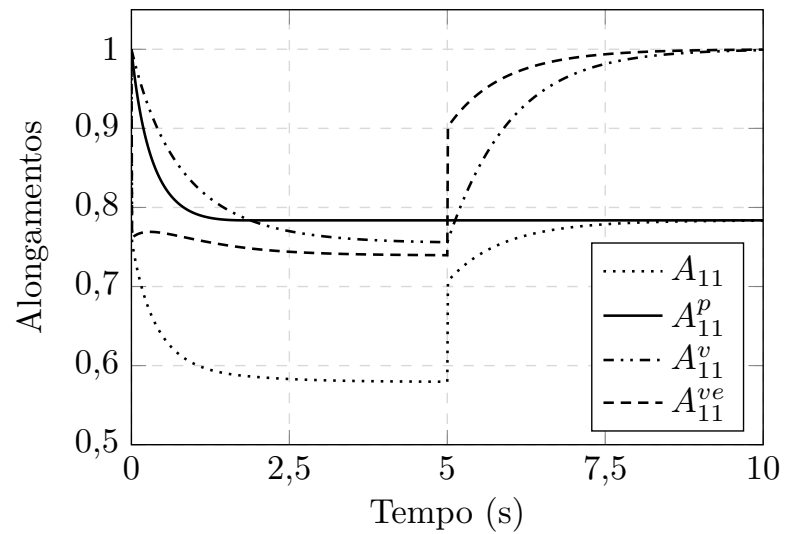

(b) Tensões

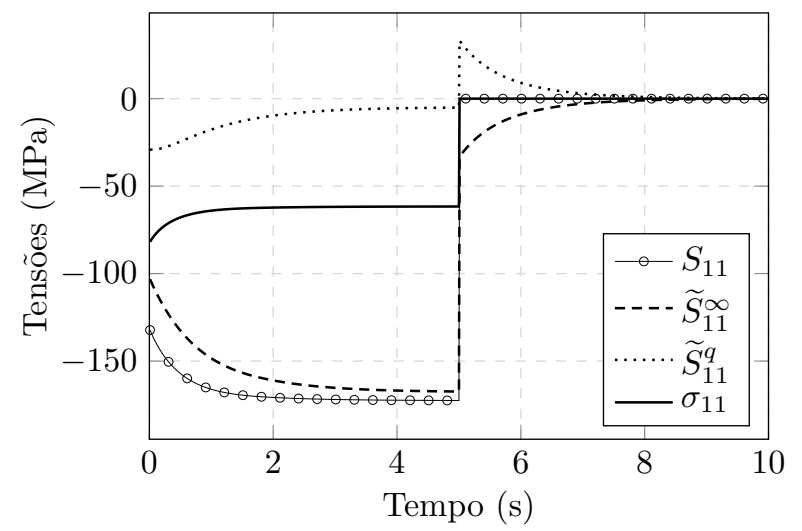


que $t \rightarrow \infty$. Na Figura 47b pode-se comparar, ainda, o comportamento ao longo do tempo das diversas parcelas de tensão envolvidas.

\subsubsection{Chapa sob cisalhamento simples}

Neste exemplo, considera-se uma chapa sob cisalhamento simples com geometria e malha semelhantes à Figura 20, e deslocamento prescrito aplicado de acordo com o gráfico da Figura 48. O instante máximo adotado foi de $t_{2}=25 \mathrm{~s}$, e o instante intermediário $t_{1}$ é variável. Foram considerados um total de 2000 passos, sendo 400 entre os instantes 0 e $\frac{1}{3} t_{1}$, 800 entre os instantes $\frac{1}{3} t_{1}$ e $\frac{5}{6} t_{1}, 400$ entre os instantes $\frac{5}{6} t_{1}$ e $t_{1}$, e 400 entre os instantes $t_{1}$ e $t_{2}$. Novamente, os valores de $\Delta t$ são variáveis entre esses intervalos.

Figura 48 - Deslocamento prescrito em chapa visco-elasto-plástica sob cisalhamento simples

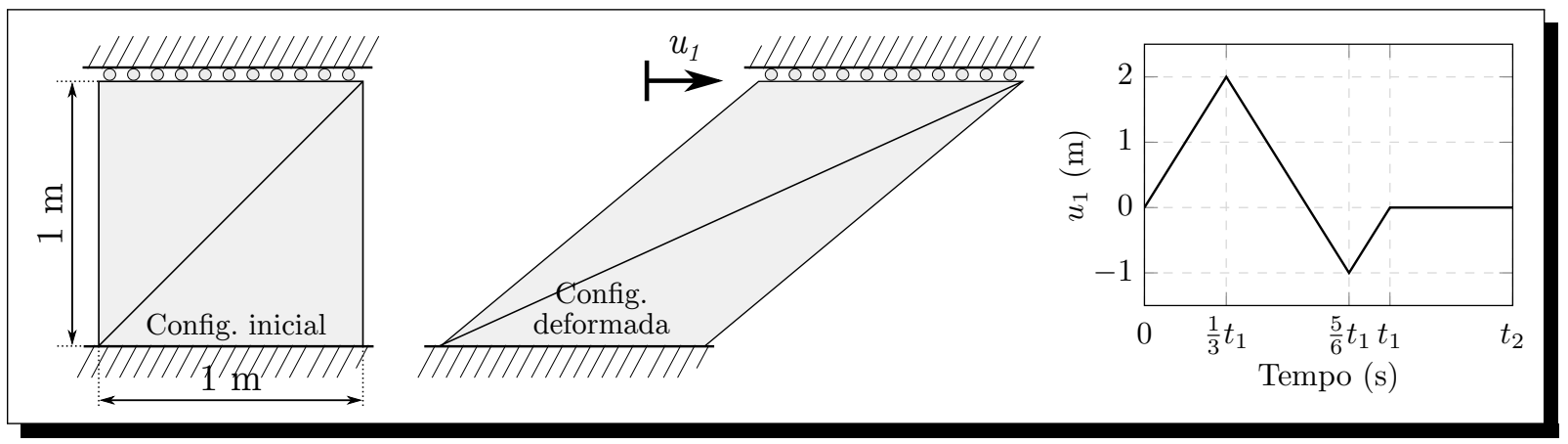

Na Figura 49 são apresentados os gráficos de tensão em função da componente $A_{12}$ para os diversos valores de $t_{1}$ considerados. Além disso, os gráficos de tensão e $\mathbf{A}^{p}$ ao longo do tempo para determinados valores de $t_{1}$ são apresentados nas Figuras $50 \mathrm{e}$ 51, observando-se um comportamento similar ao caso visco-plástico (Figuras 28 e 29), em especial no que se refere ao comportamento distinto das configurações intermediárias plásticas sob diferentes valores de taxas de deformações.

Figura 49 - Tensão-deformação para chapa visco-elasto-plástica sob cisalhamento simples

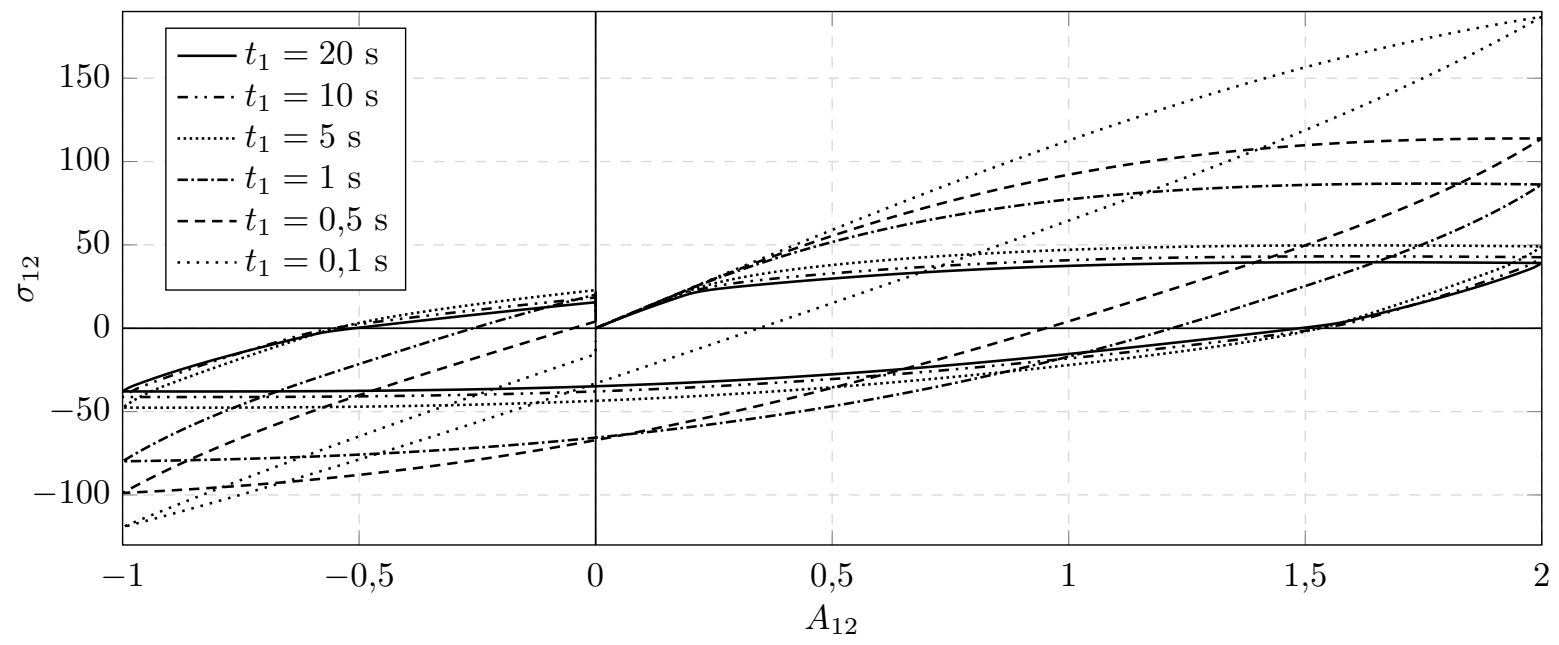


Figura 50 - Tensões de Cauchy ao longo do tempo para chapa visco-elasto-plástica sob cisalhamento simples
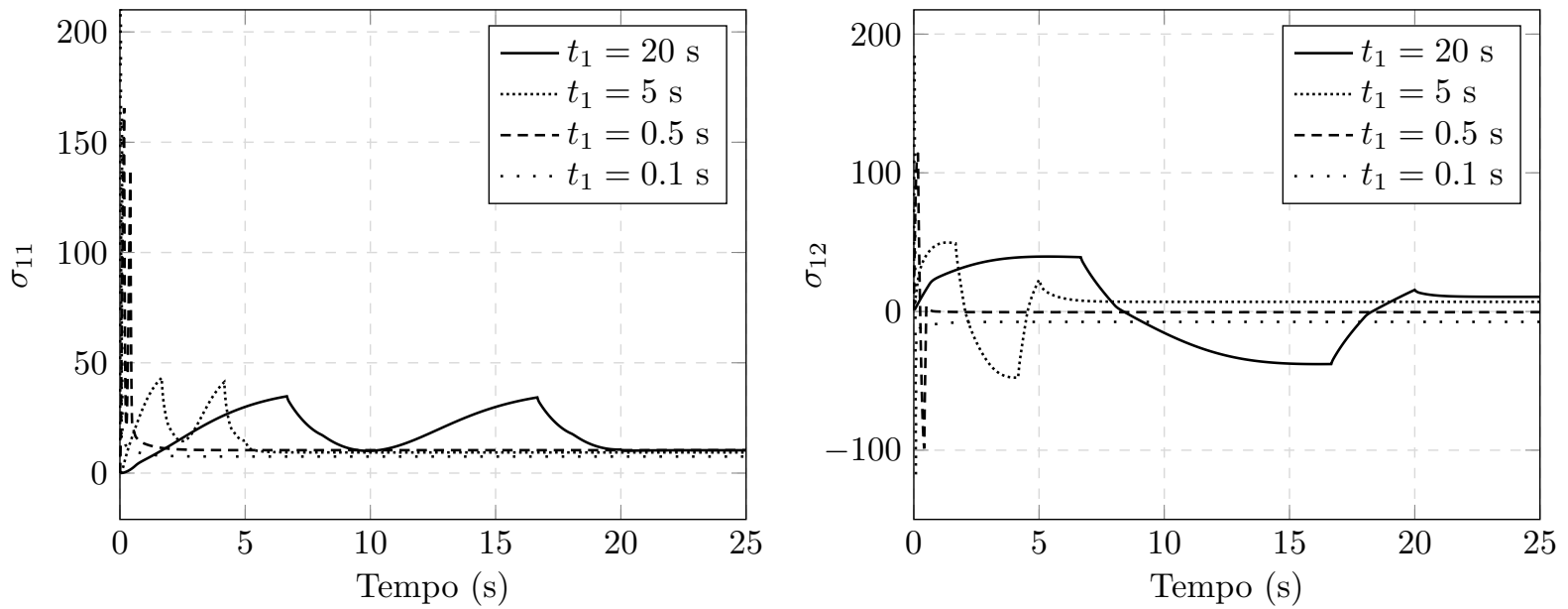

Figura 51 - Deformações cisalhantes plásticas ao longo do tempo para chapa visco-elastoplástica sob cisalhamento simples
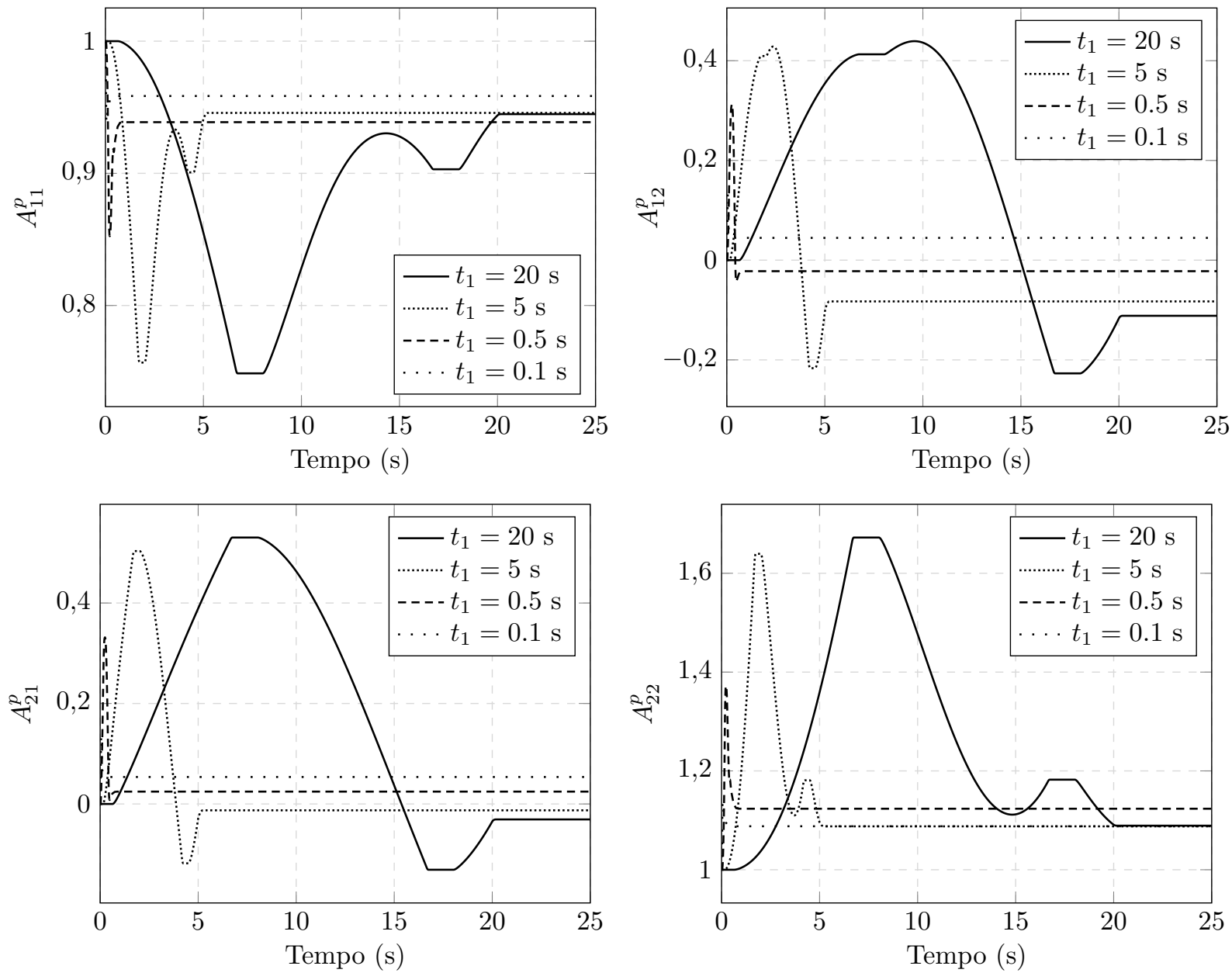


\section{Capítulo 7}

\section{A estratégia Nó-a-Segmento para problemas de contato}

Em problemas de contato sob grandes deslocamentos, onde não se pode garantir a coincidência das malhas, o modelo mais comumente adotado é o Nó-a-Segmento, que caracteriza-se por distinguir as interfaces de contato de forma que uma seja discretizada em elementos nodais (denominados nós projéteis), e outra em segmentos (denominados segmentos alvos).

Nessa técnica, cada segmento alvo é tratado como um elemento de linha curva. Dessa forma, o campo de posições definido no seu domínio (denotado por $\mathbf{y}^{S}$ ) pode ser mapeado por uma coordenada adimensional $\xi$, a partir da interpolação

$$
\mathbf{y}^{S}(\xi)=\varphi^{\alpha}(\xi) \mathbf{y}^{\alpha}
$$

onde $\mathbf{y}^{\alpha}$ e $\varphi^{\alpha}$ representam a posição e a função de forma de cada nó $\alpha$ pertencente ao segmento. Analogamente, pode-se definir o vetor tangente $\mathbf{t}$ associado a $\xi$ por meio da expressão

$$
\mathbf{t}(\xi)=\frac{\partial \mathbf{y}^{S}}{\partial \xi}(\xi)=\frac{\partial \varphi^{\alpha}}{\partial \xi}(\xi) \mathbf{y}^{\alpha}
$$

O vetor normal unitário associado a $\xi$, no caso $2 \mathrm{D}$, pode ser definido como o vetor $\mathbf{n}$ tal que $\mathbf{n} \cdot \mathbf{t}=0$ e $\|\mathbf{n}\|=1$. Essa definição, entretanto, permite dois possíveis cenários: um no qual $\mathbf{n}$ é direcionado para a parte externa do sólido, e outro para a parte interna. Neste trabalho, adota-se por padrão o primeiro caso, onde pode-se utilizar as expressões

$$
n_{1}(\xi)=-\bar{t}_{2} \quad \text { e } \quad n_{2}(\xi)=\bar{t}_{1},
$$

onde $\overline{\mathbf{t}}$ é o vetor tangente unitário, definido por:

$$
\overline{\mathbf{t}}=\frac{\mathbf{t}}{\|\mathbf{t}\|} \text {. }
$$

Para que a equação (7.3) retorne o resultado desejado, observa-se ainda a orientação do elemento finito, uma vez que essa afeta a direção de $\mathbf{t}$. Tal consideração deve ser levada em conta durante a geração da malha. Na orientação sinistrogira (Figura 52b), o vetor 
t possui direção oposta à desejada, e, portanto, a equação (7.3) resulta em um vetor $\mathbf{n}$ direcionado para a parte interna do sólido. Dessa forma, utiliza-se apenas a orientação dextrogira (Figura 52a), onde adota-se o sentido anti-horário em contornos externos do sólido e sentido horário em contornos internos (como, por exemplo, furos).

Figura 52 - Tipos de orientações dos elementos finitos de linha curva

(a) Orientação dextrogira

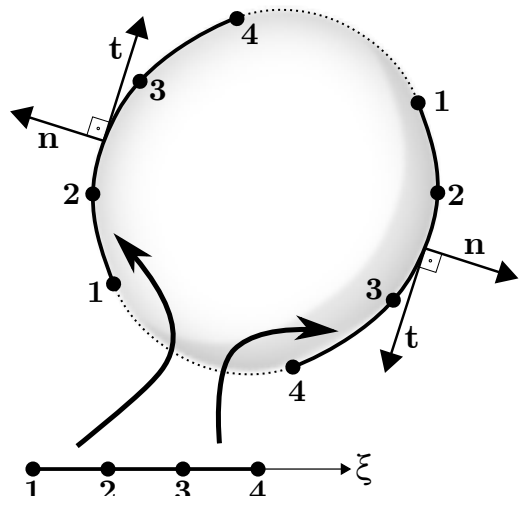

(b) Orientação sinistrogira

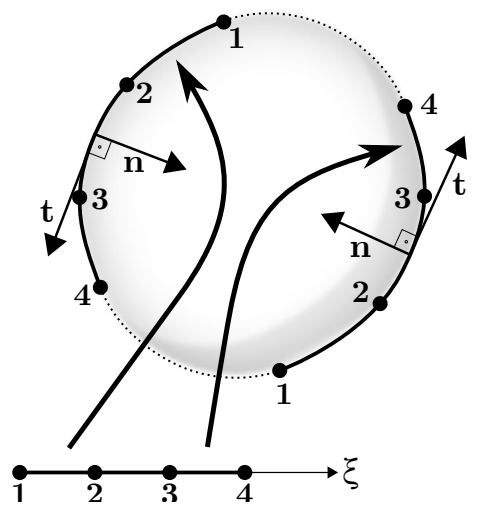

\subsection{Detecção do contato}

Para cada nó projétil com posição $\mathbf{y}^{N}$, ativa-se o contato quando existe $\xi$ pertencente ao domínio de um segmento alvo tal que a distância normal

$$
g_{n}=\left(\mathbf{y}^{N}-\mathbf{y}^{S}(\xi)\right) \cdot \mathbf{n}(\xi)
$$

seja menor ou igual a 0 . No caso sem atrito, o ponto $\xi$ onde o contato é imposto deve ser aquele que possui a menor distância normal em módulo. Para que isso ocorra, o ponto $\xi$ deve conter a projeção normal do nó projétil, conforme ilustrado na Figura 53. Em outras palavras, a seguinte condição deve ser verdadeira:

$$
g_{t}=\left(\mathbf{y}^{N}-\mathbf{y}^{S}(\xi)\right) \cdot \mathbf{t}(\xi)=0 .
$$

Figura 53 - Projeção normal de ponto projétil sobre segmento alvo

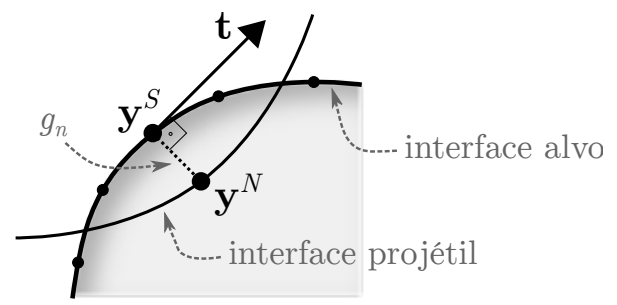

Para cada par de ponto projétil e segmento alvo, resolve-se a Equação (7.6) pelo método de Newton-Raphson (descrito na seção 3.2). Para alcançar a convergência, utiliza-se a derivada

$$
g_{t}^{\prime}=\left(\mathbf{y}^{N}-\mathbf{t}(\xi)\right) \cdot \mathbf{t}(\xi)+\left(\mathbf{y}^{N}-\mathbf{y}^{S}(\xi)\right) \cdot \mathbf{t}^{\prime}(\xi),
$$


onde

$$
\mathbf{t}^{\prime}(\xi)=\frac{\partial^{2} \varphi^{\alpha}}{\partial \xi^{2}}(\xi) \mathbf{y}^{\alpha}
$$

Na seção 7.3, o procedimento de Newton-Raphson é escrito com detalhes, bem como todo o passo-a-passo realizado no programa desenvolvido para a detecção do contato.

\subsection{Imposição das condições de contato}

Uma vez detectado o contato, a não-penetração é imposta por meio da condição $g_{n}=0$. Para introduzi-la ao sistema, os artifícios mais comumente adotados são a técnica dos multiplicadores de Lagrange e o método da penalização. O último consiste em adicionar uma restrição de mola entre o nó projétil e o respectivo ponto de contato. Esse método é relativamente mais simples e não requer a introdução de novos graus de liberdade, porém é limitado quanto à precisão, uma vez que o valor de $g_{n}$ tem sua proximidade a 0 controlada pelo valor da rigidez adotada para a mola.

Neste trabalho, utiliza-se a técnica dos multiplicadores de Lagrange, que consiste em introduzir, para cada nó projétil $N$ em contato, um novo grau de liberdade, denotado por $\lambda$, representando a força de contato atuante em $N$. A energia potencial associada a essa força é então adicionada à Equação (2.36), por meio da expressão

$$
\Pi_{\text {cont }}^{N}=\lambda g_{n} \text {. }
$$

Caso o modelo de contato adotado não inclua adesão, apenas forças de contato compressivas podem ser admitidas, isto é, o valor de $\lambda$ calculado deve ser menor ou igual a zero. Caso contrário, a condição de contato deve ser desativada.

\subsubsection{Forças de contato}

Devido à introdução de (7.9) ao sistema, adiciona-se à Equação (3.7) novas forças de contato, derivando (7.9) com relação aos graus de liberdade de posição dos nós envolvidos. No nó projétil, a força de contato é dada por

$$
\mathbf{f}_{\text {cont }}^{N}=\frac{\partial \Pi_{\text {cont }}^{N}}{\partial \mathbf{y}^{N}}=\lambda \mathbf{n}
$$

Já no segmento alvo, são aplicadas forças de contato em cada nó $\alpha$ pertencente ao elemento de linha curva, por meio da expressão

$$
\left(f_{\text {cont }}^{\alpha}\right)_{i}=\frac{\partial \Pi_{\text {cont }}^{N}}{\partial y_{i}^{\alpha}}=\lambda\left(\left(y_{k}^{N}-y_{k}^{S}\right) \frac{\partial n_{k}}{\partial y_{i}^{\alpha}}-\varphi^{\alpha} n_{i}\right),
$$

onde a derivada de $\mathbf{n}$ com relação às posições é discutida na subseção 7.2.2.

Além disso, deve-se minimizar (7.9) com relação ao grau de liberdade $\lambda$, resultando em uma nova equação a ser adicionada ao sistema, dada por

$$
\frac{\partial \Pi_{\text {cont }}^{N}}{\partial \lambda}=g_{n}=0
$$

que garante a condição de não-penetração desejada entre o nó projétil e o seu respectivo 
ponto de contato.

\subsubsection{Matriz Hessiana}

De forma a preservar a convergência do sistema global, deve-se também adicionar à matriz hessiana (seção 3.6) a parcela associada ao contato, cujos termos são apresentados na Tabela 10.

Tabela 10 - Termos da matriz hessiana local de contato usando a técnica Nó-a-Segmento e multiplicadores de Lagrange

\begin{tabular}{|r|c|c|c|}
\cline { 2 - 4 } \multicolumn{1}{c|}{} & $(\cdot) / \partial y_{j}^{N}$ & $(\cdot) / \partial y_{j}^{\beta}$ & $(\cdot) / \partial \lambda$ \\
\hline$\partial\left(f_{\text {cont }}^{N}\right)_{i} /(\cdot)$ & 0 & $\lambda \frac{\partial n_{i}}{\partial y_{j}^{\beta}}$ & $n_{i}$ \\
\hline$\partial\left(f_{\text {cont }}^{\alpha}\right)_{i} /(\cdot)$ & $\lambda \frac{\partial n_{j}}{\partial y_{i}^{\alpha}}$ & $\lambda\left(\left(y_{k}^{N}-y_{k}^{S}\right) \frac{\partial^{2} n_{k}}{\partial y_{i}^{\alpha} \partial y_{j}^{\beta}}-\frac{\partial \varphi^{\beta}}{\partial \xi} \frac{\partial n_{j}}{\partial y_{i}^{\alpha}}-\frac{\partial \varphi^{\alpha}}{\partial \xi} \frac{\partial n_{j}}{\partial y_{j}^{\beta}}\right)$ & $\left(y_{k}^{N}-y_{k}^{S}\right) \frac{\partial n_{k}}{\partial y_{i}^{\alpha}}-\varphi^{\alpha} n_{i}$ \\
\hline$\partial g_{n} /(\cdot)$ & $n_{j}$ & $\left(y_{k}^{N}-y_{k}^{S}\right) \frac{\partial n_{k}}{\partial y_{j}^{\beta}}-\varphi^{\beta} n_{j}$ & 0 \\
\hline
\end{tabular}

Usando a regra apresentada em (7.3), podemos calcular as derivadas de $\mathbf{n}$ com relação às posições a partir das derivadas de $\overline{\mathbf{t}}$, que, por sua vez, são obtidas pelas expressões

$$
\begin{gathered}
\frac{\partial \bar{t}_{k}}{\partial y_{i}^{\alpha}}=\frac{\partial \varphi^{\alpha}}{\partial \xi} \frac{\delta_{k i}}{\|\mathbf{t}\|}-\frac{t_{k}}{\|\mathbf{t}\|^{2}} \frac{\partial\|\mathbf{t}\|}{\partial y_{i}^{\alpha}} \text { e } \\
\frac{\partial^{2} \bar{t}_{k}}{\partial y_{i}^{\alpha} \partial y_{j}^{\beta}}=-\frac{\partial \varphi^{\alpha}}{\partial \xi} \frac{\delta_{k i}}{\|\mathbf{t}\|^{2}} \frac{\partial\|\mathbf{t}\|}{\partial y_{j}^{\beta}}-\frac{t_{k}}{\|\mathbf{t}\|^{2}} \frac{\partial^{2}\|\mathbf{t}\|}{\partial y_{i}^{\alpha} \partial y_{j}^{\beta}}-\frac{\partial \varphi^{\beta}}{\partial \xi} \frac{\delta_{k j}}{\|\mathbf{t}\|^{2}} \frac{\partial\|\mathbf{t}\|}{\partial y_{i}^{\alpha}}+\frac{2 t_{k}}{\|\mathbf{t}\|^{3}} \frac{\partial\|\mathbf{t}\|}{\partial y_{i}^{\alpha}} \frac{\partial\|\mathbf{t}\|}{\partial y_{j}^{\beta}}
\end{gathered}
$$

onde

$$
\begin{gathered}
\frac{\partial\|\mathbf{t}\|}{\partial y_{i}^{\alpha}}=\frac{\partial \varphi^{\alpha}}{\partial \xi} \frac{t_{i}}{\|\mathbf{t}\|} \\
\frac{\partial^{2}\|\mathbf{t}\|}{\partial y_{i}^{\alpha} \partial y_{j}^{\beta}}=\frac{\partial \varphi^{\alpha}}{\partial \xi} \frac{\partial \varphi^{\beta}}{\partial \xi}\left(\frac{\delta_{i j}}{\|\mathbf{t}\|}-\frac{t_{i} t_{j}}{\|\mathbf{t}\|^{3}}\right)
\end{gathered}
$$

e $\delta$ é a função delta de Kronecker, definida em (A.13).

\subsection{Algoritmo}

No algoritmo 2, apresenta-se um esboço dos passos para detecção ou desativação do contato. Esse algoritmo é introduzido após a obtenção do equilíbrio, isto é, após a linha 38 do algoritmo 1. Caso seja detectado um novo par de contato, ou a necessidade de desativar alguma condição, retorna-se à linha 8 do algoritmo 1 e resolve-se novamente o sistema com a introdução das novas condições.

Em outros tipos de abordagem, o algoritmo para deteç̧ão ou desativação do contato pode ser inserido dentro do procedimento de Newton-Raphson global (por exemplo, após a linha 36 do algoritmo 1), sendo a checagem, portanto, realizada em cada iteração. 
Tal procedimento dispensa a necessidade de reiniciar o sistema diversas vezes em um mesmo passo de tempo, muitas vezes aumentando a rapidez da solução, porém, em certos casos pode dificultar a convergência. Dessa forma, optou-se neste trabalho pela primeira alternativa.

O cálculo das forças de contato e da hessiana, bem como a imposição das mesmas ao sistema, pode ser feita, por exemplo, antes da aplicação dos deslocamentos prescritos (linha 30 do algoritmo 1).

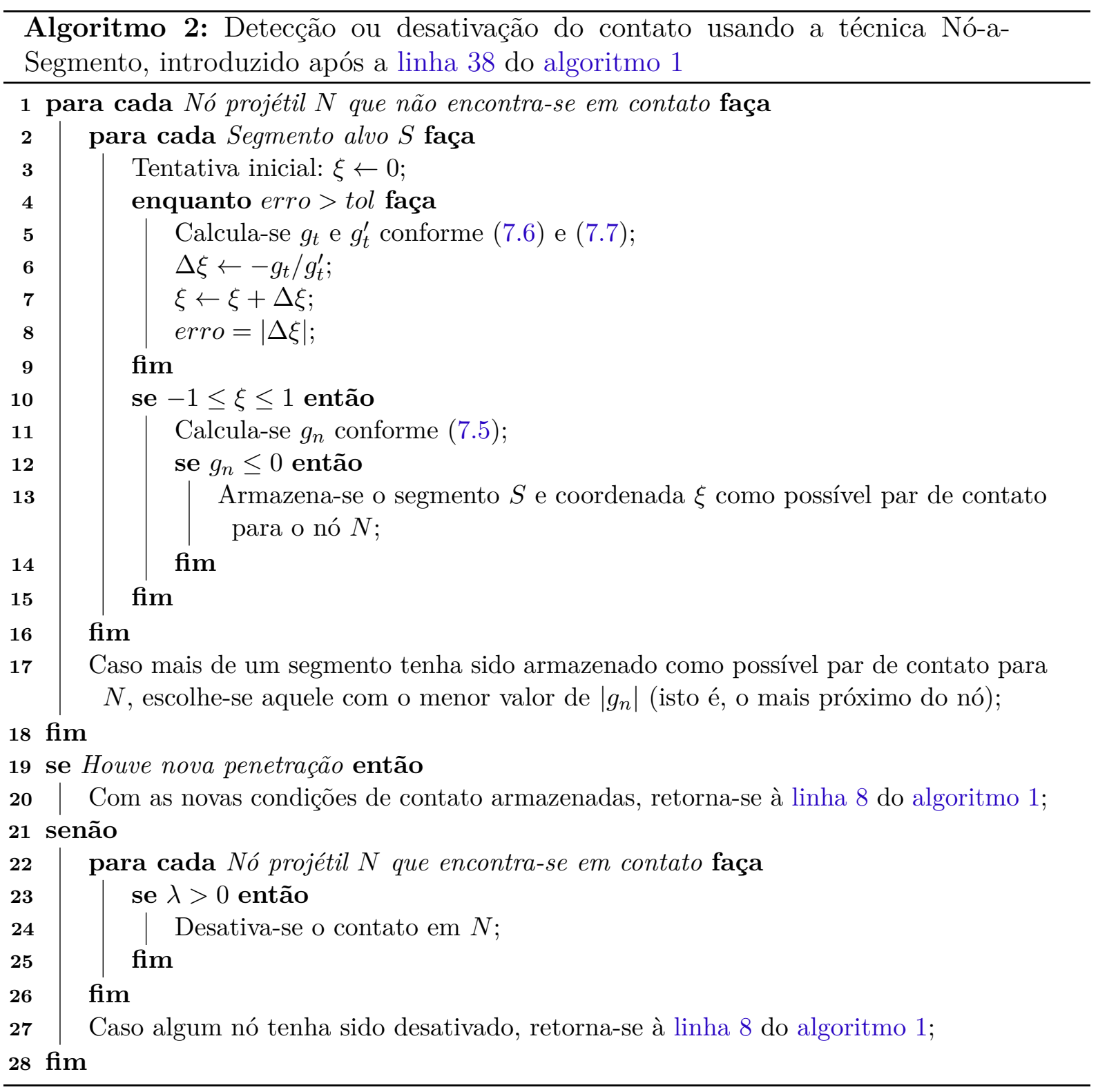

\subsection{Exemplo de verificação: Arco deslizante}

Este exemplo, extraído de Piedade Neto (2013) e baseado em Yang, Laursen e Meng (2005), apresenta contato entre dois corpos deformáveis sujeitos a grandes deslocamentos e tem como objetivo validar o algoritmo desenvolvido em uma análise estática. Dois arcos circulares conforme a Figura 54 são compelidos entre si por meio de deslocamento prescrito 
horizontal $\Delta u=25$ ao longo de 200 passos de 0.125 cada, aplicado nos apoios do arco superior. O material para ambos é considerado neo-Hookeano, com parâmetros $E=698.56$ e $\nu=0.32$, que equivale às constantes de Lamé $\Lambda=255$ e $\mu=264$.6. Utiliza-se a técnica Nó-a-Segmento para o contato, onde as interfaces projétil e alvo consideradas são indicadas também na Figura 54.

Figura 54 - Exemplo do arco deslizante, extraído de Piedade Neto (2013)

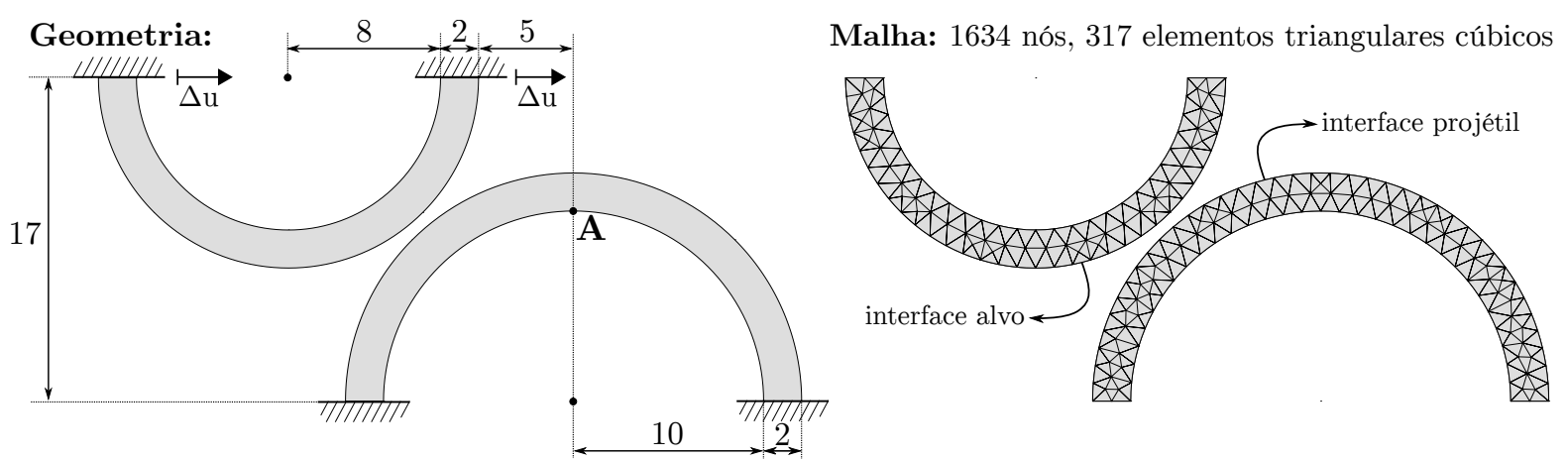

O problema é interessante por apresentar um efeito denominado snap-through, típico de situações instáveis, onde há mudanças bruscas de posições entre passos de tempo consecutivos. Esse comportamento é problemático do ponto de vista numérico, pois pode gerar problemas de convergência no método de Newton-Raphson a depender do número de passos adotado na análise.

Figura 55 - Configurações deformadas do arco deslizante e tensão de von Mises

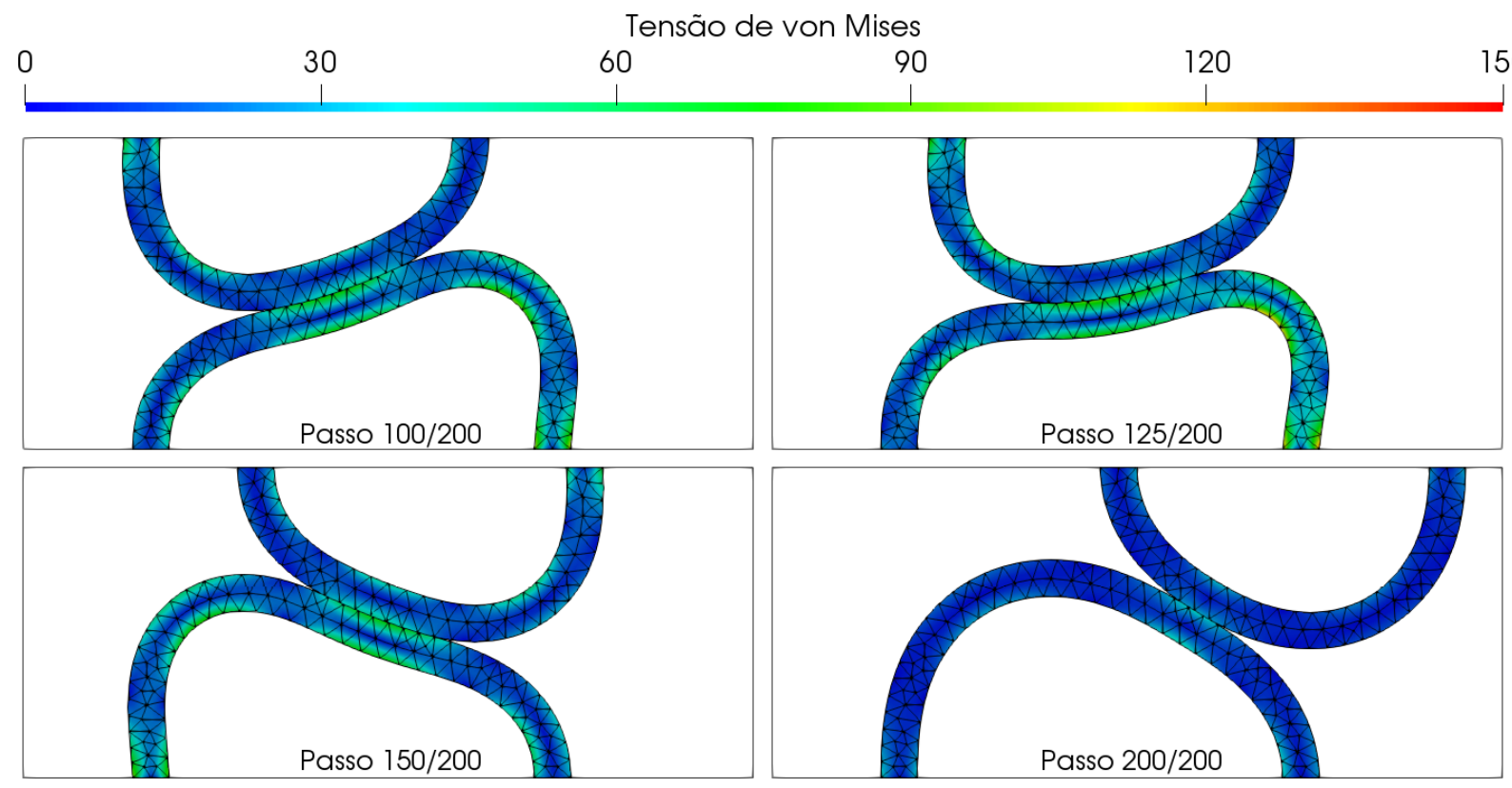

Tanto as configurações deformadas quanto os valores da tensão de von Mises, ilustradas na Figura 55, mostram uma boa proximidade com o trabalho de Piedade Neto (2013), onde o problema é resolvido com a técnica Mortar de contato. Na Figura 56, apresentam-se os gráficos de deslocamento vertical e tensão de Cauchy horizontal para o ponto A, onde o fenômeno do snap-through pode ser observado mais claramente no 
trecho descontínuo das curvas. Novamente, observa-se uma excelente concordância entre os resultados obtidos e a fonte.
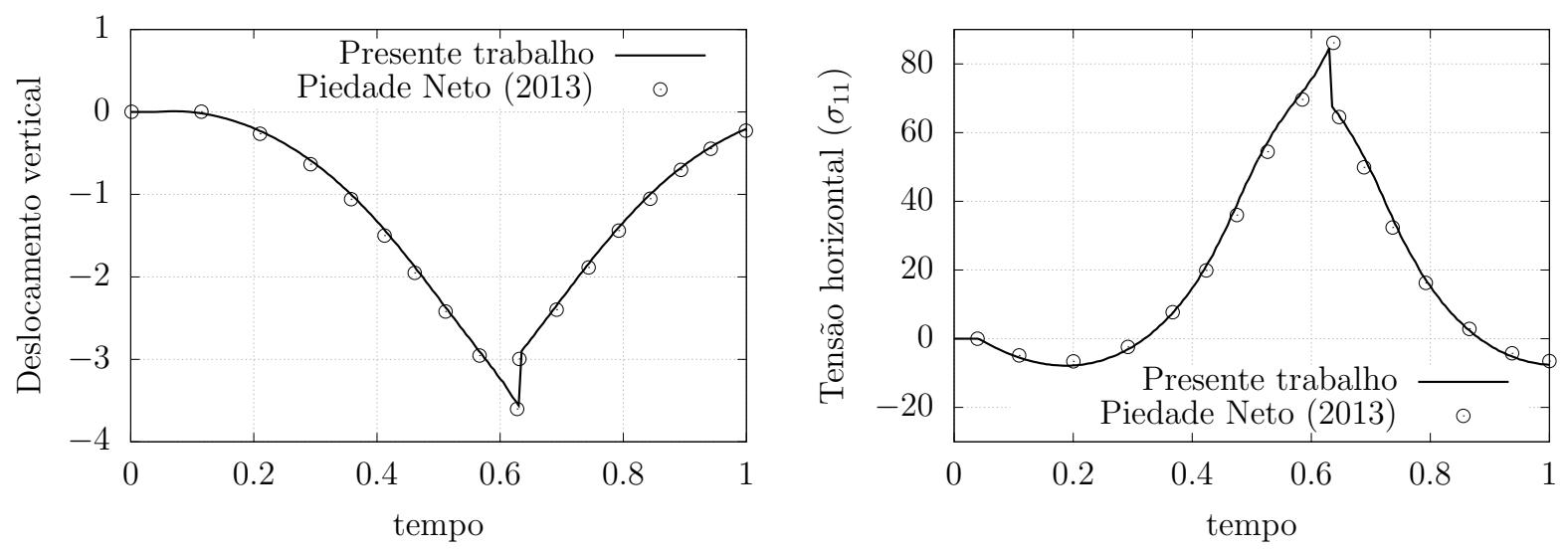

Figura 56 - Gráficos de deslocamento vertical e tensão horizontal no ponto A 



\section{Capítulo 8}

\section{Exemplos numéricos propostos}

Para mostrar as potencialidades do código desenvolvido, propõem-se neste capítulo diversos exemplos numéricos utilizando os modelos constitutivos descritos, sendo alguns inspirados em processos de manufatura existentes, incluindo situações de contato. São simulados também exemplos dinâmicos ${ }^{1}$, tendo em vista a ampla gama de aplicações envolvendo esse tipo de análise. O objetivo desses exemplos é tornar mais clara a influência de cada parâmetro constitutivo no comportamento do material, bem como analisar a resposta do modelo numérico a diferentes discretizações temporais e diferentes parâmetros para o integrador temporal de Newmark. Foram adotados em todos os exemplos elementos triangulares de aproximação cúbica, e 7 pontos de integração.

\subsection{Bloco parcialmente carregado}

Este exemplo consiste na análise de um bloco com material polimérico sujeito a um carregamento compressivo distribuído parcialmente conforme a Figura 57, sendo considerado estado plano de deformações. Devido à simetria, apenas metade do problema é discretizado, sendo adotada uma malha regular composta por 200 elementos e 961 nós. Três modelos constitutivos foram considerados: visco-elasto-plástico (conforme parâmetros da Tabela 9), visco-elástico e visco-plástico. O tempo máximo de análise $t_{1}$ é variável, sendo o número de passos de tempo fixo em 10000.

Figura 57 - Geometria e malha para exemplo de bloco parcialmente carregado

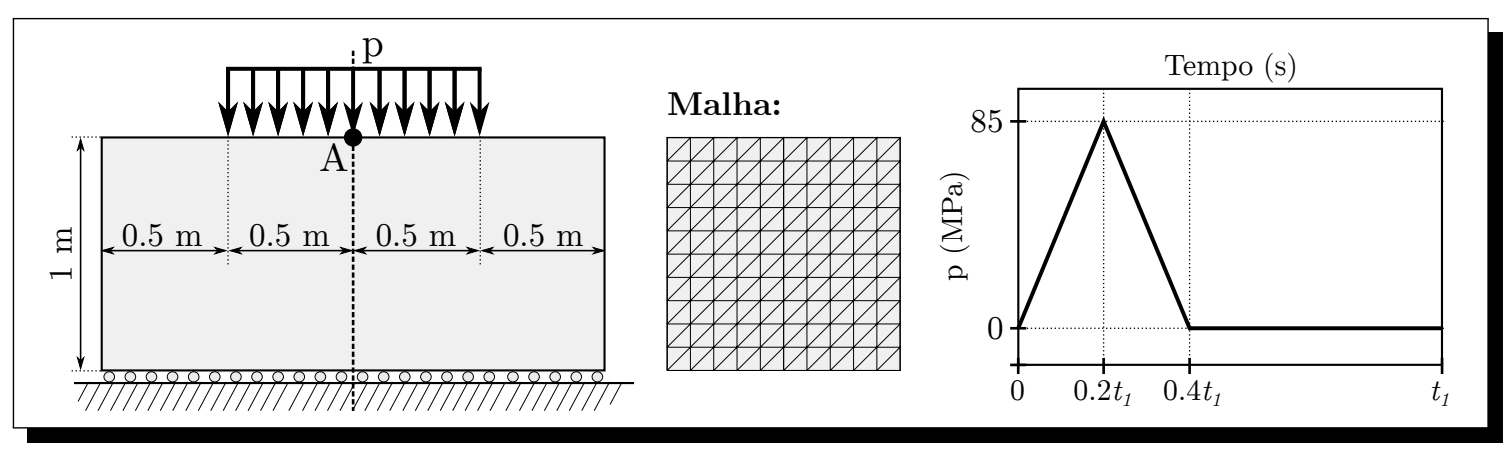

$\overline{1}$ Nos exemplos em que a análise dinâmica não for explicitamente mencionada, considera-se análise quase-estática. 


\subsubsection{Modelo visco-elástico}

Para o caso visco-elástico, consideram-se os parâmetros dados na Tabela 11. Os resultados são comparados ainda com os modelos hiperelásticos associados I e II, definidos conforme a Tabela 7 (nota-se que, neste caso, o modelo hiperelástico I é equivalente ao III, uma vez que $\left.\Lambda^{e}=0\right)$.

Tabela 11 - Parâmetros para material visco-elástico do tipo polimérico

\begin{tabular}{ccccc}
\hline$\Lambda^{\infty}(\mathrm{MPa})$ & $\mu^{\infty}(\mathrm{MPa})$ & $\Lambda^{e}(\mathrm{MPa})$ & $\mu^{e}(\mathrm{MPa})$ & $\eta(\mathrm{MPa} \cdot \mathrm{s})$ \\
\hline 320 & 80 & 0 & 40 & 50 \\
\hline
\end{tabular}

Na Figura 58 apresenta-se o gráfico de carregamento aplicado e deslocamento vertical no ponto A. Seguindo o comportamento já observado em exemplos do Capítulo 5, as taxas mais baixas e mais altas aproximam-se dos modelos hiperelásticos I e II, respectivamente, causando uma redução no caráter histerético das curvas.

Figura 58 - Diagrama de força-deslocamento para o bloco visco-elástico

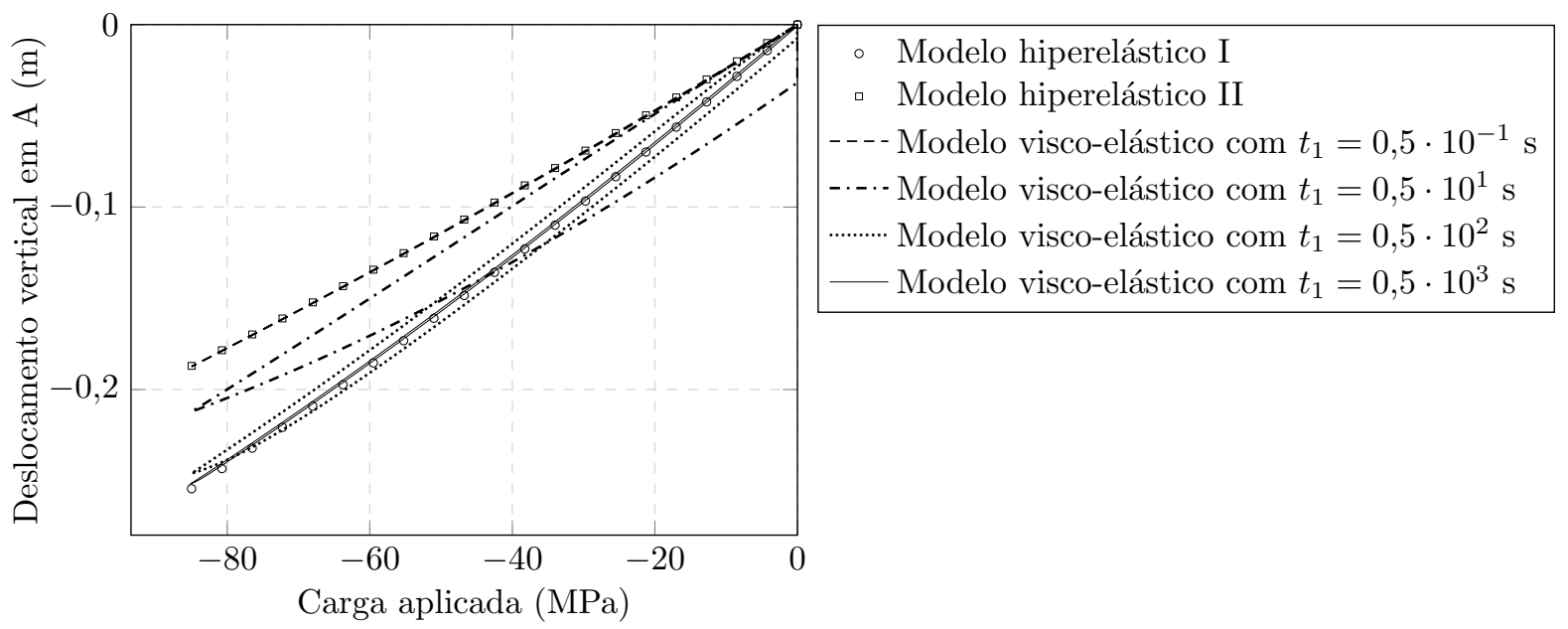

Na Figura 59 são mostradas ainda as configurações deformadas na etapa de carregamento máximo para diferentes valores de $t_{1}$, bem como os mapas de cores da componente $E_{22}^{v}$ das deformações viscosas.

Figura 59 - Configurações deformadas na etapa de carga máxima em bloco visco-elástico

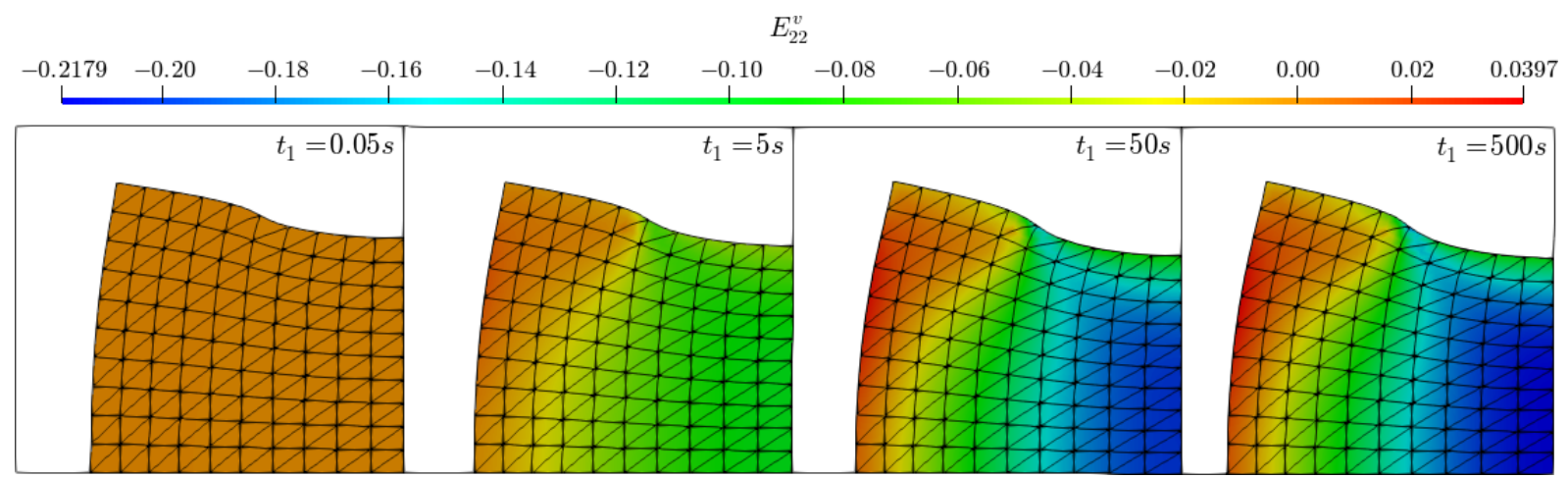


É interessante observar que, embora os valores máximos e mínimos de deslocamentos ocorram nos casos de valores extremos de taxas de carregamento, os casos intermediários apresentam deslocamentos maiores que os demais, em valor absoluto, durante alguns trechos do processo de descarregamento e após a remoção completa da carga, como mostram as curvas de retorno da Figura 60.

Figura 60 - Deslocamento ao longo do tempo para o bloco visco-elástico

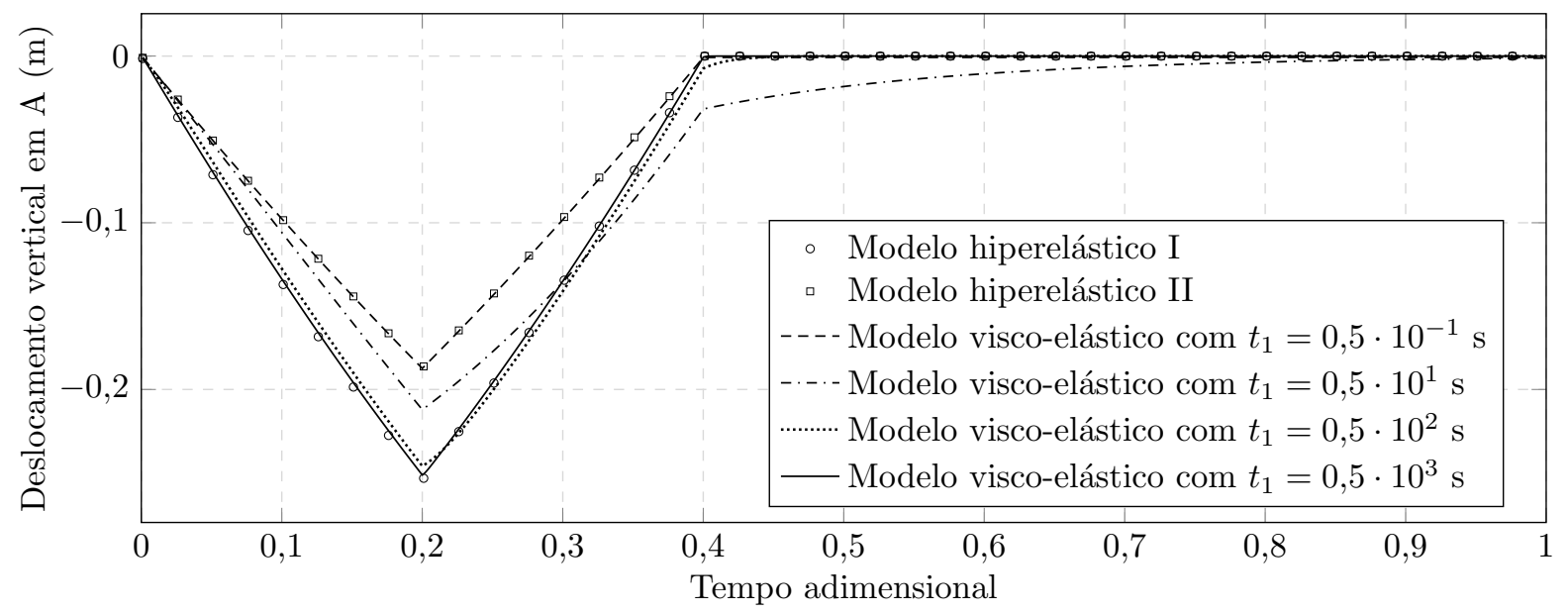

\subsubsection{Modelo visco-plástico}

Para o caso visco-plástico, o material adotado é o polimérico da Tabela 2 (PET orientado), com parâmetros de Perzyna e Norton dados na Tabela 5.

As curvas deslocamento vs. carga distribuída são apresentadas na Figura 61 para diversos valores de $t_{1}$. Observa-se que taxas de carregamento mais baixas provocam maiores deslocamentos tanto no instante de carregamento máximo quanto no descarregamento, ao passo que taxas mais altas reduzem o caráter histerético das curvas, fazendo com que as respostas se aproximem de um comportamento hiperelástico.

Figura 61 - Diagrama de força-deslocamento para o caso visco-plástico

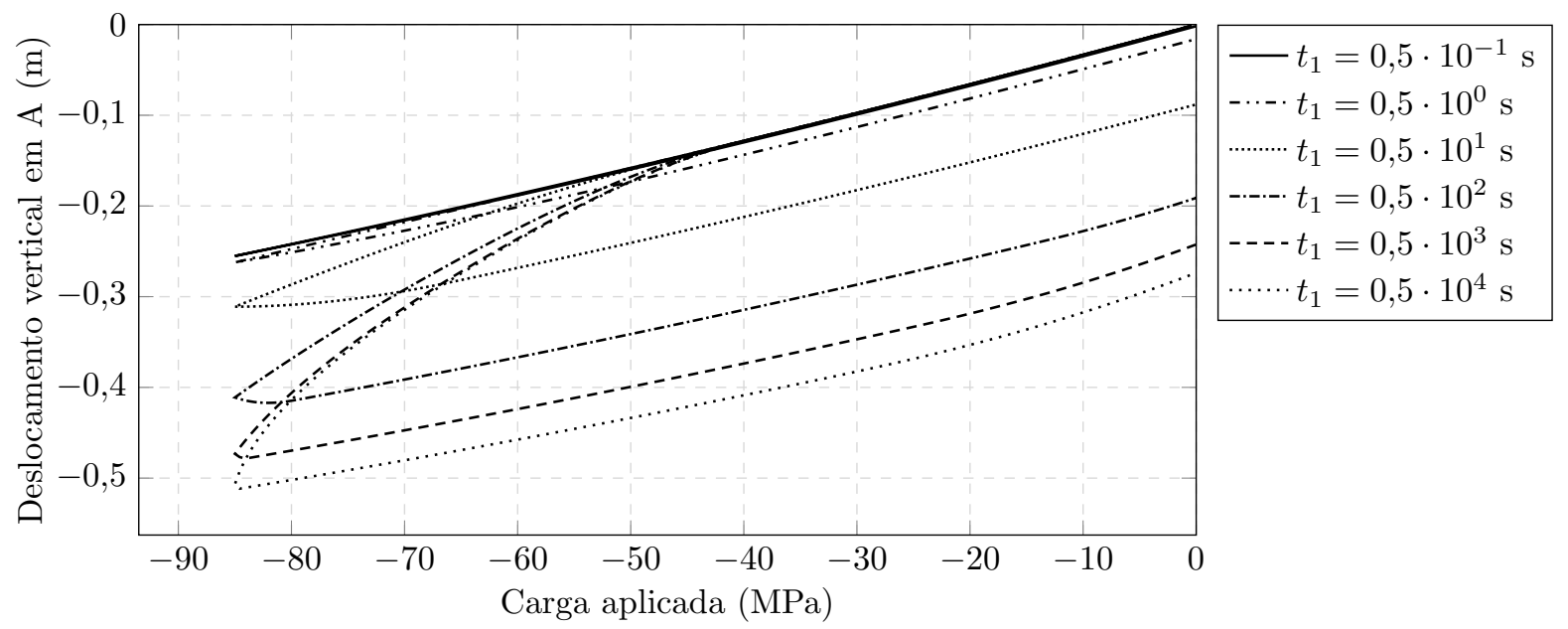


Na Figura 62 são mostradas as configurações deformadas nas etapas de carregamento máximo e no instante final analisado para diversos valores de $t_{1}$, onde é notável a redução das deformações plásticas à medida que $t_{1}$ é reduzido.

Figura 62 - Configurações deformadas para o caso visco-plástico

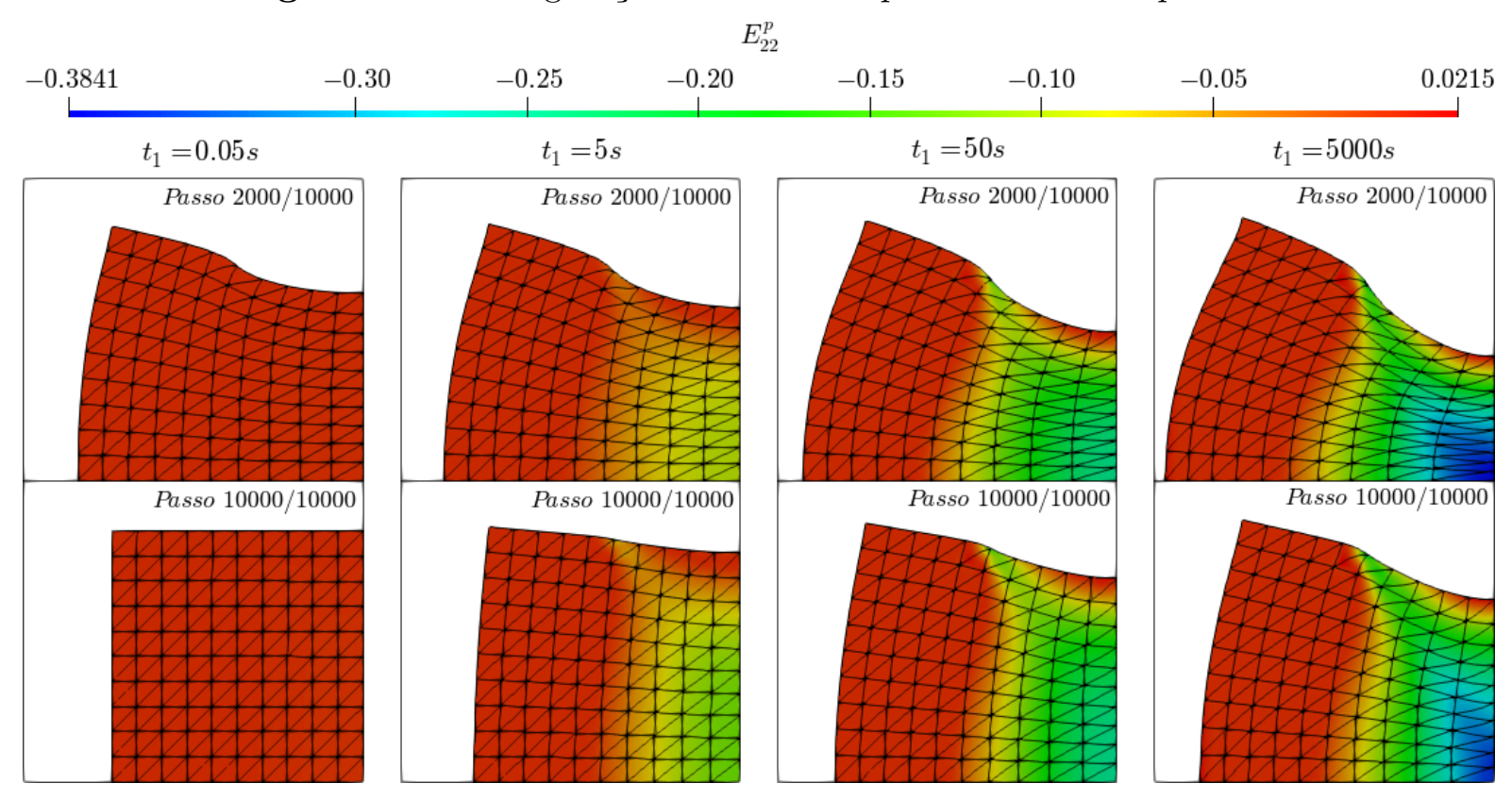

\subsubsection{Modelo visco-elasto-plástico}

Neste caso, os parâmetros do material são aqueles apresentados na Tabela 9. Conforme mencionado na subseção 4.2.6, a aplicação do algoritmo implícito de Euler provoca erros na propriedade da conservação do volume inelástico. Para investigar a dependência da discretização temporal sobre esses erros no regime de grandes deformações, realiza-se neste exemplo uma análise de convergência para os valores do jacobiano plástico $\left(J^{p}\right)$ e viscoso $\left(J^{v}\right)$, apresentada na Figura 63, onde foi adotado o tempo máximo $t_{1}=$ $0,5 \cdot 10^{4} \mathrm{~s}$, discretizado por meio de quatro incrementos de tempo $(\Delta t)$ diferentes: $2 \mathrm{~s}, 1 \mathrm{~s}, 0,5$ s e 0,25 s. A inclinação média das retas apresentadas nos gráficos é de 1,149944643 para o

Figura 63 - Análise de convergência para jacobianos plásticos e viscosos

(a) Jacobiano plástico

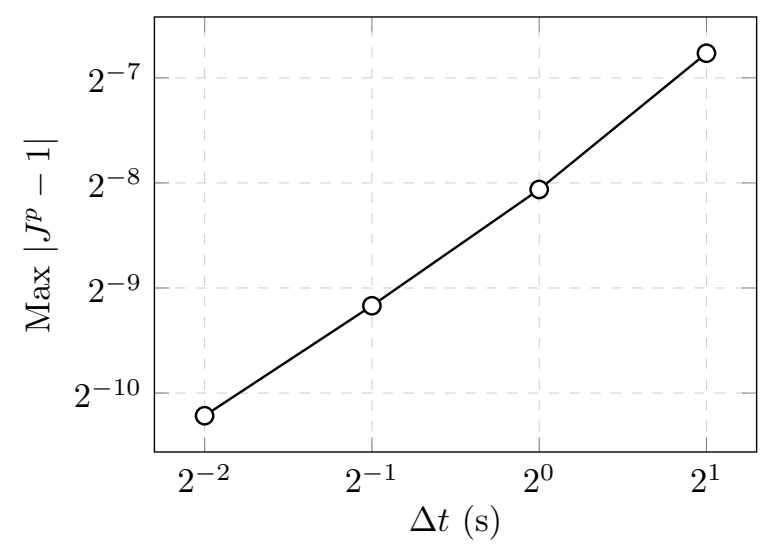

(b) Jacobiano viscoso

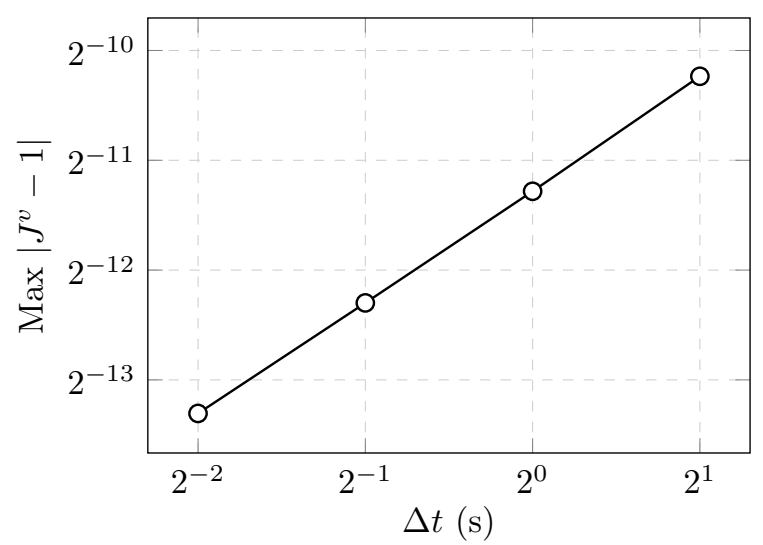


Jacobiano plástico e 1,023697852 para o Jacobiano viscoso, indicando convergência próxima de primeira ordem. Na prática, isso implica que o erro é reduzido aproximadamente pela metade à medida que o número de passos é dobrado.

Os gráficos de força vs. deslocamento obtidos são mostrados na Figura 64a, para diversos valores de $t_{1}$, e na Figura 64b, para as diversas discretizações temporais adotadas na análise de convergência, mantendo-se $t_{1}=0,5 \cdot 10^{4} \mathrm{~s}$. Nesse último o gráfico é ampliado, sendo mostrada apenas a região pŕoxima ao máximo valor absoluto da carga, de modo a evidenciar as diferenças entre os resultados (especialmente entre os casos de $\Delta t=0,25 \mathrm{~s}$ e $\Delta t=0,5 \mathrm{~s})$.

Figura 64 - Diagrama de força vs. deslocamento para o bloco visco-elasto-plástico

(a) Variando $t_{1}$

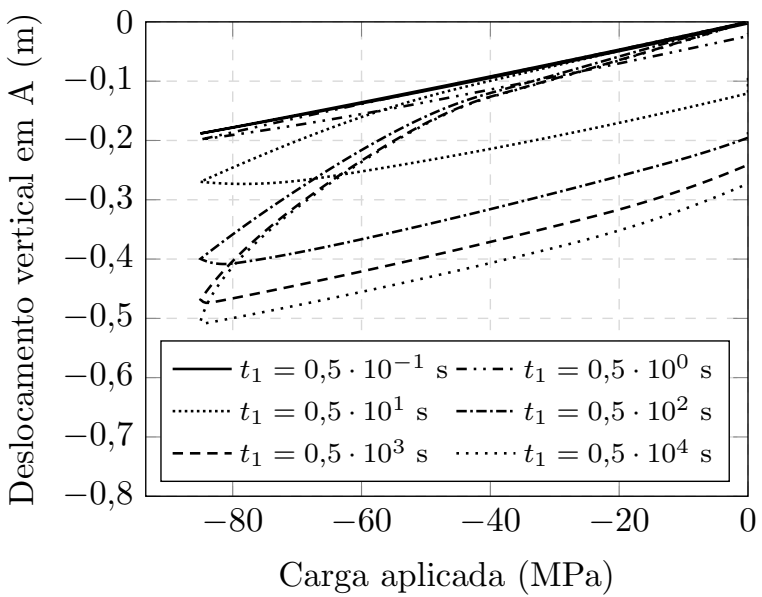

(b) Variando $\Delta t\left(t_{1}=0,5 \cdot 10^{4} \mathrm{~s}\right)$

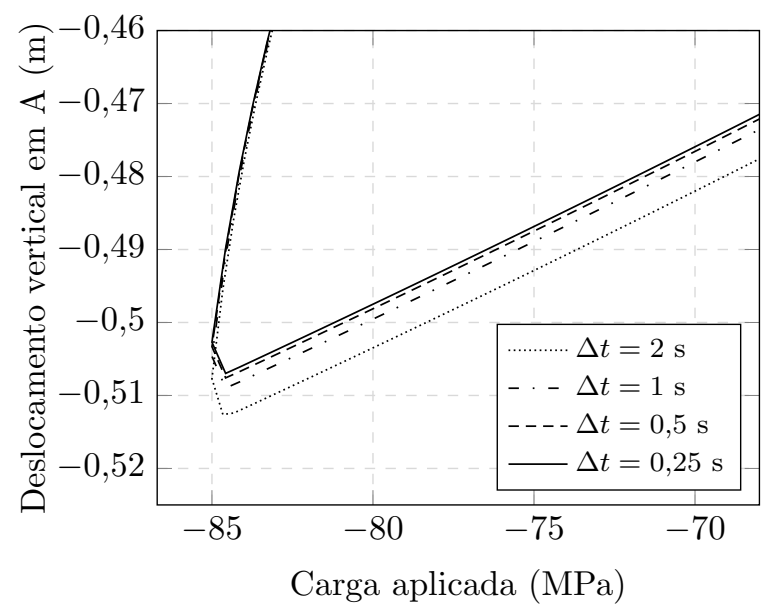

O deslocamento do ponto A ao longo do tempo é apresentado na Figura 65, tanto para o caso visco-elasto-plástico como para o caso visco-plástico. Nesse último, não se observa evolução a partir do instante de remoção completa da carga para nenhum valor

Figura 65 - Deslocamento ao longo do tempo para o bloco visco-elasto-plástico

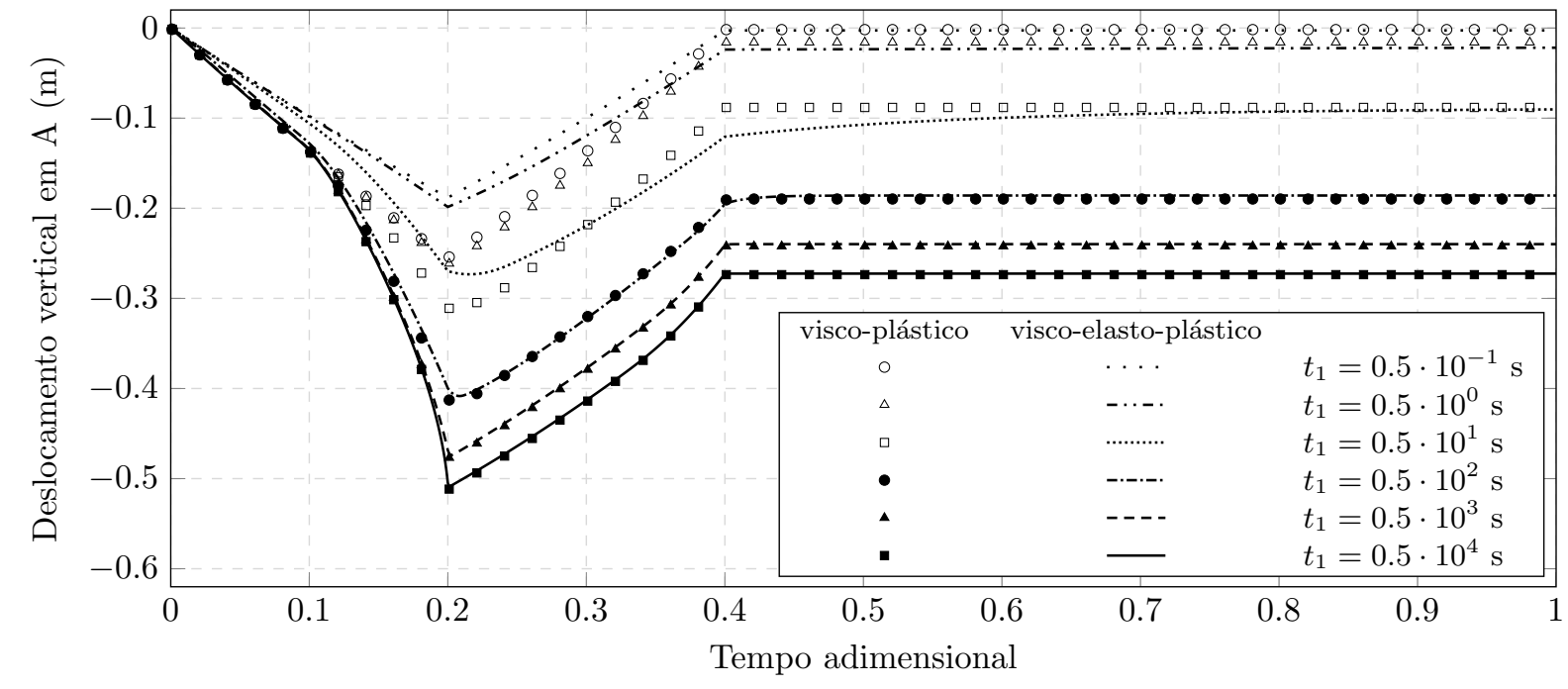


de $t_{1}$. O mesmo ocorre no caso visco-elasto-plástico para taxas muito altas e muito baixas, uma vez que, nesses casos, as respostas se aproximam das visco-plásticas e hiperelásticas, respectivamente. No entanto, para valores intermediários de $t_{1}$, o retorno para a configuração plástica não é imediato, devido ao fato de que ainda há evoluções na parcela viscosa. As configurações deformadas nas etapas de carregamento máximo e no instante final analisado são mostradas na Figura 66, onde também é apresentada a distribuição de deformação viscosa $E_{22}^{v}$.

Figura 66 - Distribuição de deformação viscosa sobre a configuração deformada em diferentes instantes

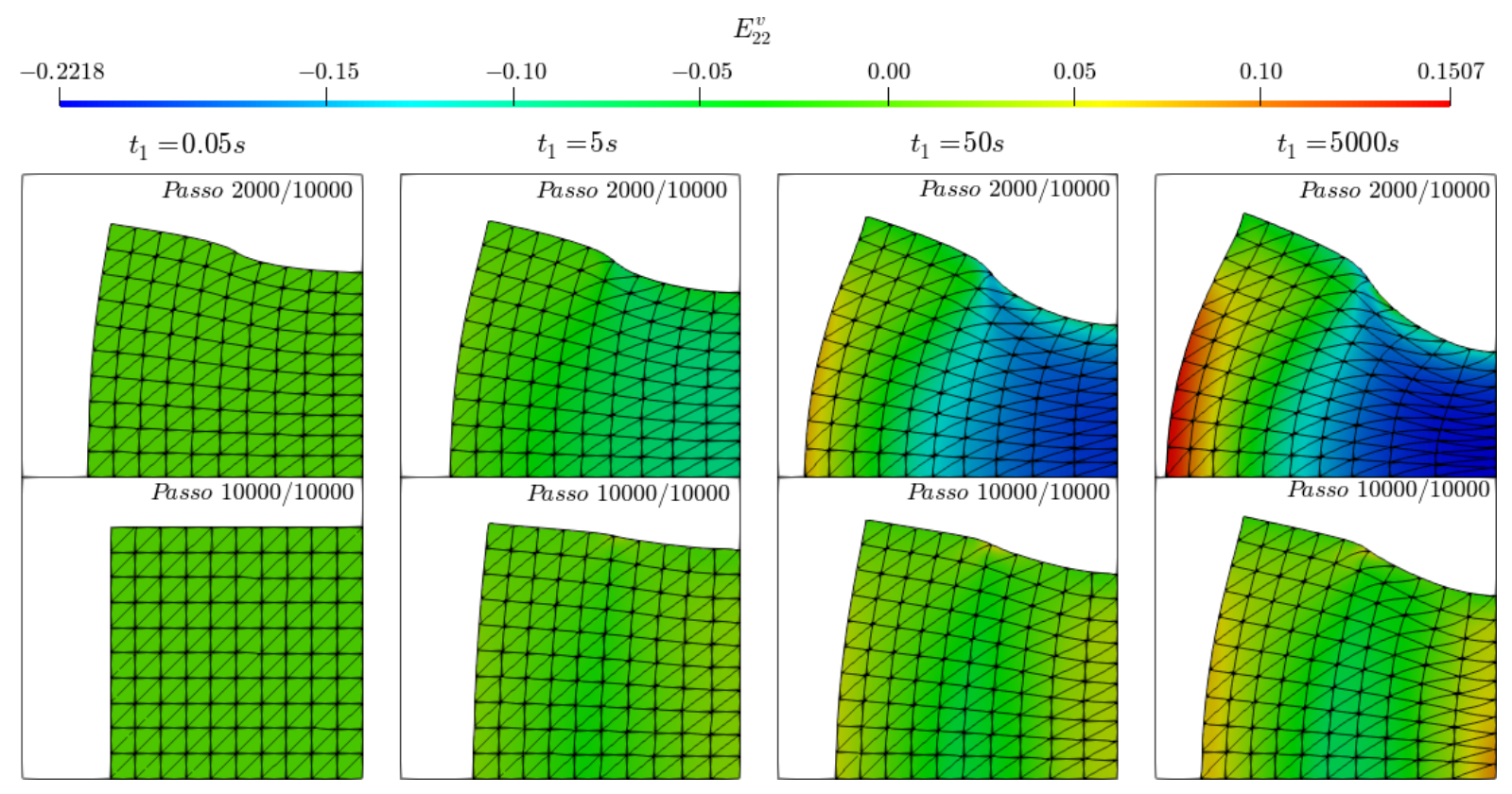

\subsection{Análise dinâmica de viga em balanço}

Este exemplo tem como intuito caracterizar a resposta dinâmica dos diversos modelos constitutivos apresentados, apresentando os efeitos de amortecimento provocados pelo comportamento dissipativo. Considera-se uma viga com geometria e carregamento dados por Bathe, Ramm e Wilson (1975), conforme a Figura 67, e estado plano de tensões. Adota-se uma malha regular com 610 nós e 120 elementos triangulares de aproximação cúbica. A massa específica é $\rho=10^{-6} l b \cdot s^{2} / i n^{4}$, e o intervalo de tempo adotado $\Delta t=$

Figura 67 - Geometria e malha para exemplo dinâmico de viga em balanço

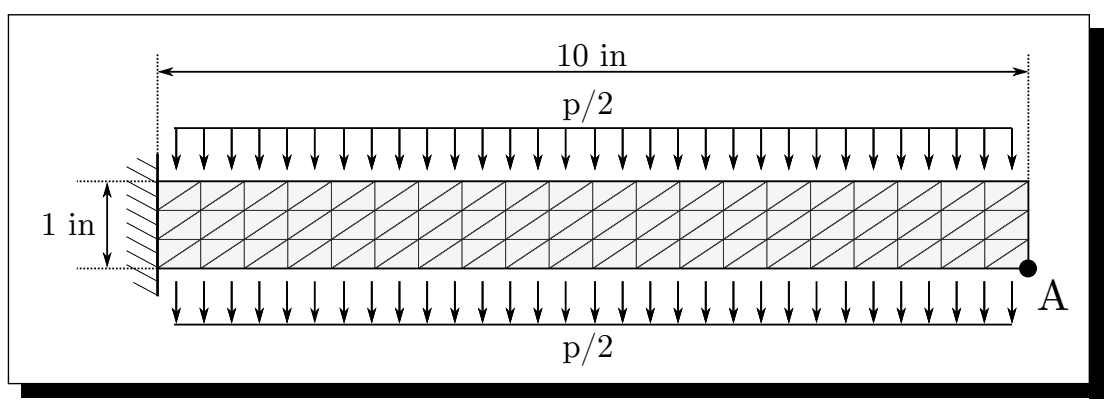


$4,1667 \cdot 10^{-5} \mathrm{~s}$, com tempo máximo e número de passos variados. A carga distribuída nas análises dinâmicas é constante e aplicada subitamente.

Inicialmente, o exemplo é comparado com o trabalho de Bathe, Ramm e Wilson (1975) para o caso hiperelástico, considerando lei Neo-Hookeana com $\Lambda=3333,33 \mathrm{lb} / \mathrm{in}^{2}$ e $\mu=5000 \mathrm{lb} / \mathrm{in}^{2}$. Na Figura 68 são apresentados os resultados dessa análise para os casos dinâmico e estático, onde nesse último considerou-se carga crescente até $\mathrm{p}=10 \mathrm{lb} /$ in . Nota-se em ambos os casos boa concordância com a referência.

Figura 68 - Verificação do exemplo dinâmico de viga em balanço com o trabalho de Bathe, Ramm e Wilson (1975) para o caso hiperelástico

(a) Caso dinâmico

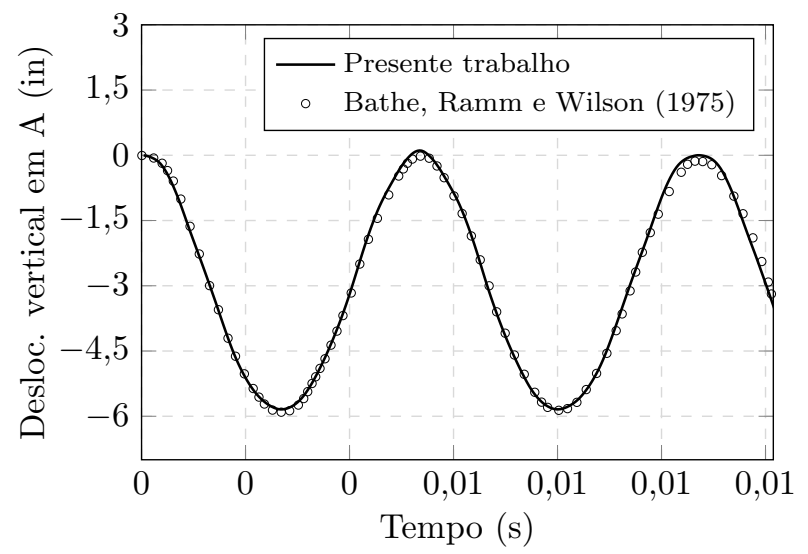

(b) Caso estático

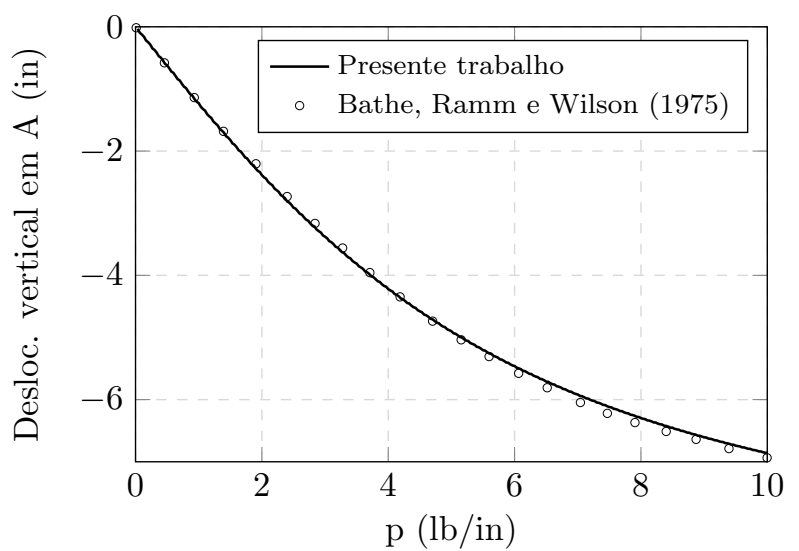

\subsubsection{Modelo visco-elástico}

No caso visco-elástico, considera-se um material com os parâmetros da Tabela 12, e os materiais hiperelásticos associados I, II e III, definidos conforme a Tabela 7.

Tabela 12 - Material visco-elástico para exemplo dinâmico de viga em balanço

\begin{tabular}{ccccc}
\hline$\Lambda^{\infty}\left(\mathrm{lb} / \mathrm{in}^{2}\right)$ & $\mu^{\infty}\left(\mathrm{lb} / \mathrm{in}^{2}\right)$ & $\Lambda^{e}\left(\mathrm{lb} / \mathrm{in}^{2}\right)$ & $\mu^{e}\left(\mathrm{lb} / \mathrm{in}^{2}\right)$ & $\eta\left(\mathrm{s} \cdot \mathrm{lb} / \mathrm{in}^{2}\right)$ \\
\hline 3333.33 & 5000 & 3333.33 & 5000 & variável \\
\hline
\end{tabular}

Na Figura 69 observa-se, como esperado, que a resposta dinâmica para valores muito elevados de $\eta$ se aproxima do modelo hiperelástico II, pelo menos nos instantes iniciais do carregamento. Já para menores valores de $\eta$, embora haja uma semelhança aos modelos hiperelásticos I e III (especialmente o primeiro), o erro fica bem mais evidenciado à medida que o tempo avança.

Para valores intermediários de $\eta$, o modelo constitutivo provoca um visível efeito de amortecimento na resposta dinâmica, conforme mostrado na Figura 70. Nota-se que tal amortecimento é mais acentuado entre os valores $\eta=4$ e $\eta=8$, diminuindo à medida que a viscosidade se distancia dessa faixa (seja para valores maiores ou menores).

Para demostrar melhor esse efeito, apresenta-se na Figura 71a o gráfico de viscosidade e tempo de estabilização para os casos analisados, sendo considerado como tempo de 
Figura 69 - Comportamento dinâmico de viga visco-elástica para valores extremos de $\eta$
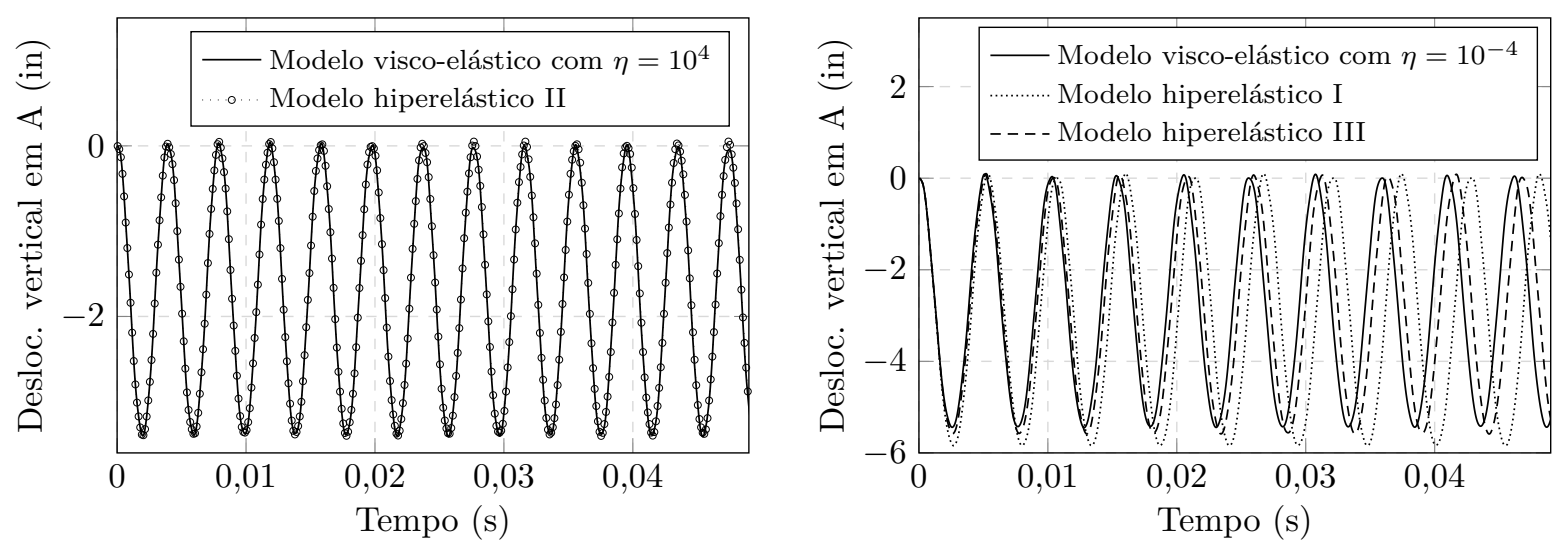

estabilização aquele a partir do qual todas as amplitudes de deslocamento são menores que uma certa tolerância (no caso, $2 \cdot 10^{-2}$ ). Para valores de $\eta$ abaixo de 4 e acima de 8 , as inclinações médias das retas obtidas no gráfico se aproximam de -1 e 1, respectivamente. No primeiro caso, isso implica que o tempo de estabilização é aproximadamente dobrado à medida que $\eta$ é reduzido pela metade. Já no segundo caso, o tempo é aproximadamente dobrado à medida que $\eta$ é dobrado. Em ambos os casos, o valor limite é $+\infty$, uma vez que os casos limites se associam aos modelos hiperelásticos, onde não há dissipação.

Figura 70 - Comportamento dinâmico de viga visco-elástica para diferentes valores de $\eta$
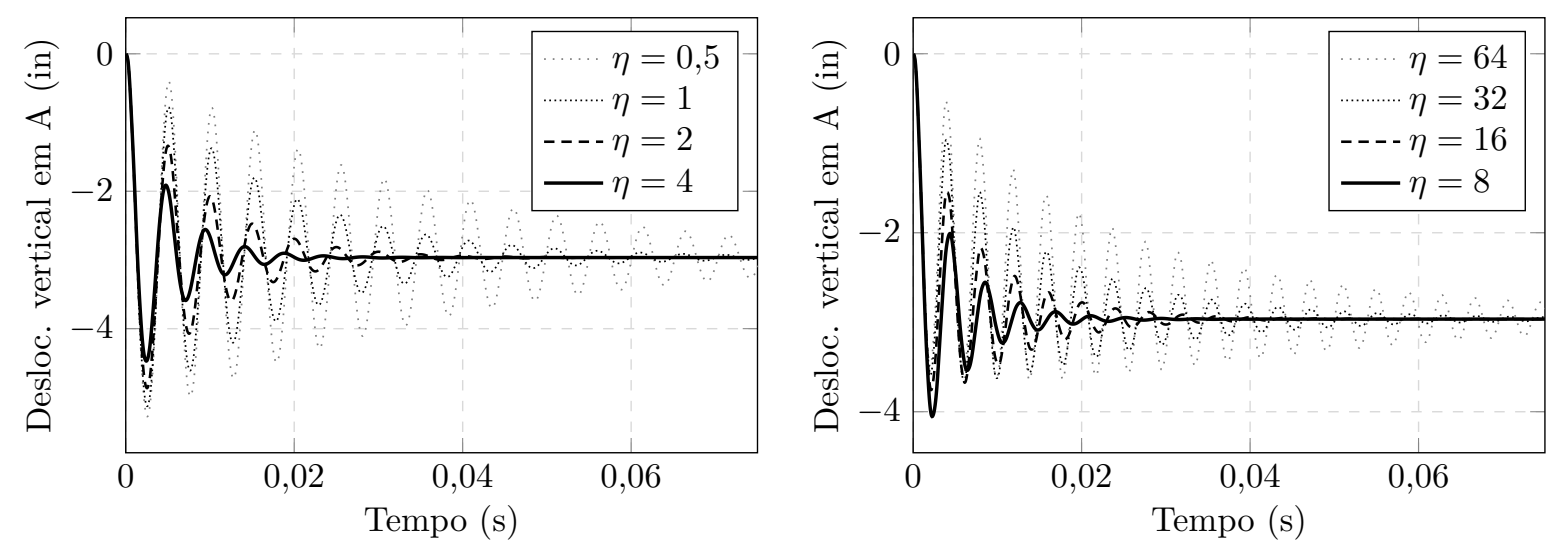

Já a frequência tende a convergir para valor em torno de $2^{7.62} \mathrm{~Hz}$ à medida em que $\eta$ diminui e para valor em torno de $2^{7.98} \mathrm{~Hz}$ à medida em que $\eta$ aumenta (ver Figura 71b). Os valores de frequência nesse caso são calculados apenas no intervalo entre os dois primeiros picos de deslocamento. É interessante observar ainda que as regiões de valores mínimos da Figura 71a (entre $\eta=4$ e $\eta=8$ ) nesse caso abrangem o ponto de inflexão da curva de frequência.

\subsubsection{Modelo visco-plástico}

No caso visco-plástico, considera-se o material dado na Tabela 13. Os gráficos de deslocamento ao longo do tempo são mostrados na Figura 72 para diversos valores de $\eta_{p}$. 
Figura 71 - Gráficos de tempo de estabilização e frequência de viga visco-elástica para diferentes valores de $\eta$, em escala logarítmica na base 2

(a) Tempo de estabilização

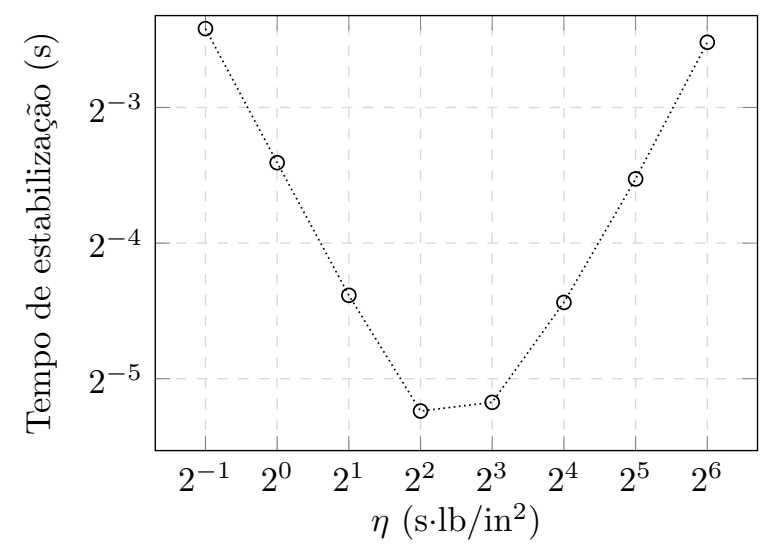

(b) Frequência

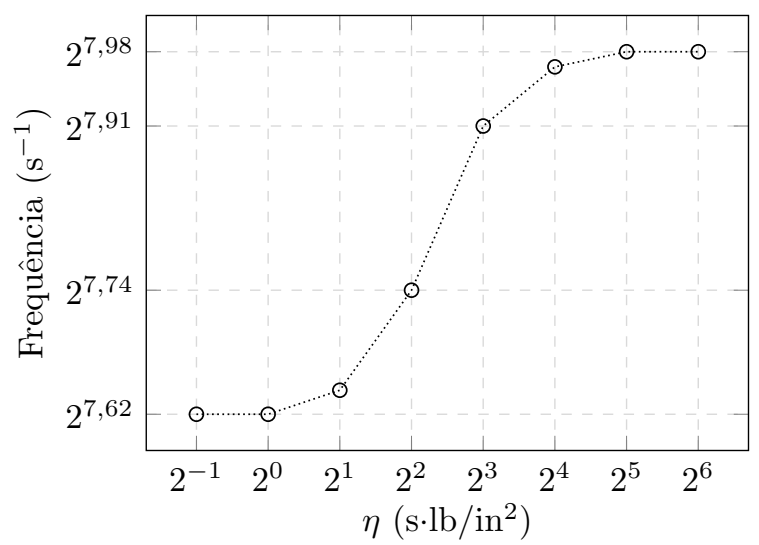

Embora nesse caso haja um efeito de amortecimento inicial, observa-se que a viga continua vibrando indefinidamente. Essa vibração se deve à parcela não-dissipativa do modelo, que, nesse caso, corresponde à parcela hiperelástica.

Tabela 13 - Material visco-plástico para exemplo dinâmico de viga em balanço

\begin{tabular}{cccccccc}
\hline$\Lambda^{e}\left(\mathrm{lb} / \mathrm{in}^{2}\right)$ & $\mu^{e}\left(\mathrm{lb} / \mathrm{in}^{2}\right)$ & $\sigma_{Y}\left(\mathrm{lb} / \mathrm{in}^{2}\right)$ & $c\left(\mathrm{lb} / \mathrm{in}^{2}\right)$ & $b$ & $\eta_{p}(\mathrm{~s})$ & $\alpha_{p}\left(\mathrm{lb} / \mathrm{in}^{2}\right)$ & $m$ \\
\hline 3333.33 & 5000 & 800 & 100 & 2 & variável & 800 & 1 \\
\hline
\end{tabular}

Figura 72 - Comportamento dinâmico de viga visco-plástica para diferentes valores de $\eta_{p}$

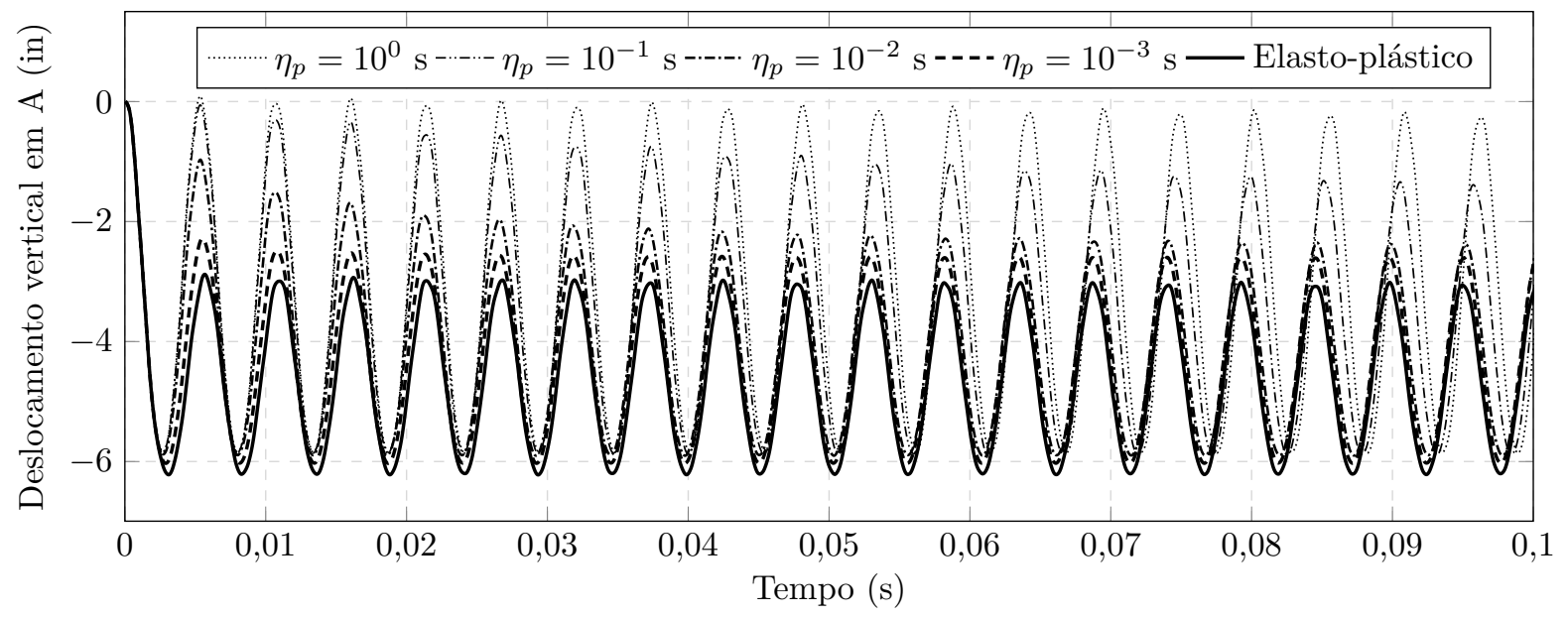

\subsubsection{Modelo visco-elasto-plástico}

No caso visco-elasto-plástico, considera-se o material representado na Tabela 14. Os gráficos de deslocamento ao longo do tempo são mostrados na Figura 73 para $\eta_{p}=10^{-3} \mathrm{~s}$ e diversos valores de $\eta$. Assim como no caso visco-elástico, o modelo constitutivo provoca um efeito de amortecimento, fazendo com que os deslocamentos se estabilizem em um determinado valor (resposta estacionária). Entretanto, enquanto no modelo visco-elástico 
Tabela 14 - Material visco-elasto-plástico para exemplo dinâmico de viga em balanço

\begin{tabular}{cccccc}
\hline \multicolumn{5}{c}{ Parâmetros visco-elásticos } \\
\hline$\Lambda^{\infty}\left(\mathrm{lb} / \mathrm{in}^{2}\right)$ & $\mu^{\infty}\left(\mathrm{lb} / \mathrm{in}^{2}\right)$ & $\Lambda^{e}\left(\mathrm{lb} / \mathrm{in}^{2}\right)$ & $\mu^{e}\left(\mathrm{lb} / \mathrm{in}^{2}\right)$ & $\eta\left(\mathrm{s} \cdot \mathrm{lb} / \mathrm{in}^{2}\right)$ \\
3333.33 & 5000 & 3333.33 & 5000 & variável \\
\hline \multicolumn{2}{c}{ Parâmetros de encruamento } & Parâmetros de Perzyna/Norton \\
\hline$\sigma_{Y}\left(\mathrm{lb} / \mathrm{in}^{2}\right)$ & $c\left(\mathrm{lb} / \mathrm{in}^{2}\right)$ & $b$ & $\eta_{p}(\mathrm{~s})$ & $\alpha_{p}\left(\mathrm{lb} / \mathrm{in}^{2}\right)$ & $m$ \\
800 & 100 & 2 & variável & 800 & 1 \\
\hline
\end{tabular}

esse valor é independente de $\eta$, nesse caso ele varia de acordo com o parâmetro de viscosidade adotado.

Figura 73 - Comportamento dinâmico de viga visco-elasto-plástica para $\eta_{p}=10^{-3}$ s e diferentes valores de $\eta$
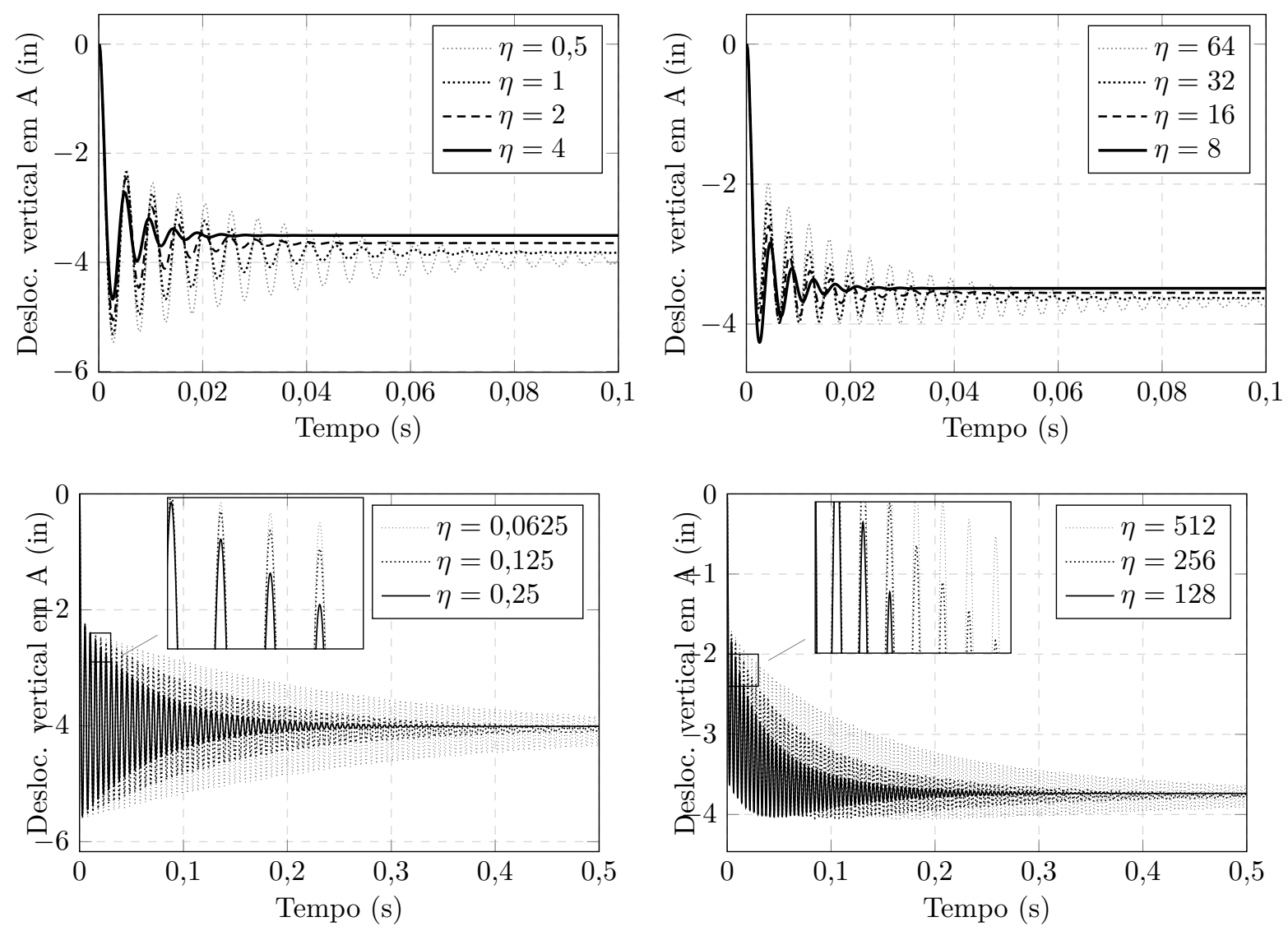

Observa-se na Figura 74a que o tempo de dissipação comporta-se de maneira semelhante ao modelo visco-elástico, obtendo seu valor mínimo entre os casos $\eta=4$ e $\eta=8$. Os deslocamentos finais também são menores para essa faixa de viscosidade, conforme mostrado na Figura 74b. Nesse gráfico, observa-se ainda que a inclinação da reta tende a diminuir conforme $\eta$ aumenta (para valores acima de 8) ou diminui (para valores abaixo de 4), indicando uma possível convergência para um determinado valor limite em cada um dos casos. Tal valor demonstra ser maior para valores menores de $\eta$, como era esperado, uma vez que o modelo hiperelástico II, associado à valores infinitamente grandes de $\eta$, apresenta deslocamentos menores que o I ou III, associados à valores infinitamente 
pequenos de $\eta$.

Figura 74 - Gráficos de tempo de estabilização e deslocamento final de viga visco-elasto-plástica para $\eta_{p}=10^{-3}$ s diferentes valores de $\eta$, em escala logarítmica na base 2

(a) Tempo de estabilização

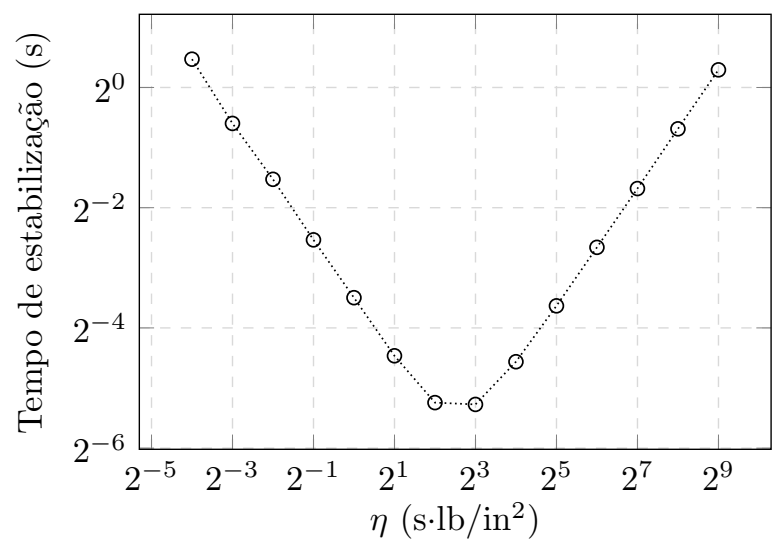

(b) Deslocamento vertical final, em módulo

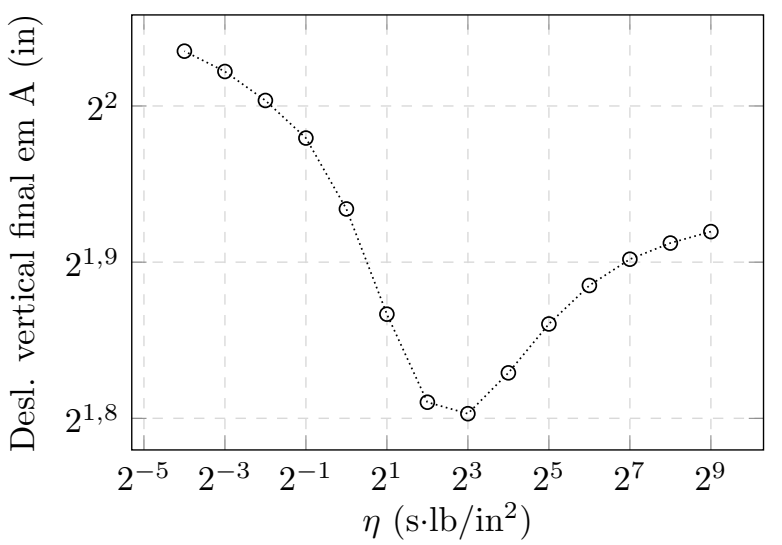

Na Figura 75a são mostrados os deslocamentos ao longo do tempo, dessa vez considerando $\eta=8$ s e variando o valor de $\eta_{p}$. Observa-se um tempo de estabilização similar em todos os casos, porém, novamente, há variação nos valores de deslocamento estacionários. Conforme esperado, a resposta quando $\eta_{p}$ tende a zero se assemelha ao caso com plasticidade independente de taxa (denotado no gráfico como $\eta_{p} \rightarrow 0$ ). A diferença entre o deslocamento final obtido nesse caso, denotado por $u_{\text {est }}(0)$, e o deslocamento final para cada valor de $\eta_{p}$, denotado por $u_{e s t}\left(\eta_{p}\right)$, é apresentado na Figura $75 \mathrm{~b}$, em escala logarítmica na base 10. Naturalmente, esse valor tende a zero à medida que $\eta_{p}$ é reduzido. Além disso, à medida que $\eta_{p}$ cresce, a ordem de convergência tende a diminuir, indicando a existência de um valor limite mínimo de deslocamento final.

Figura 75 - Resposta de viga visco-elasto-plástica para $\eta=8$ s e diferentes valores de $\eta_{p}$

(a) Deslocamento ao longo do tempo

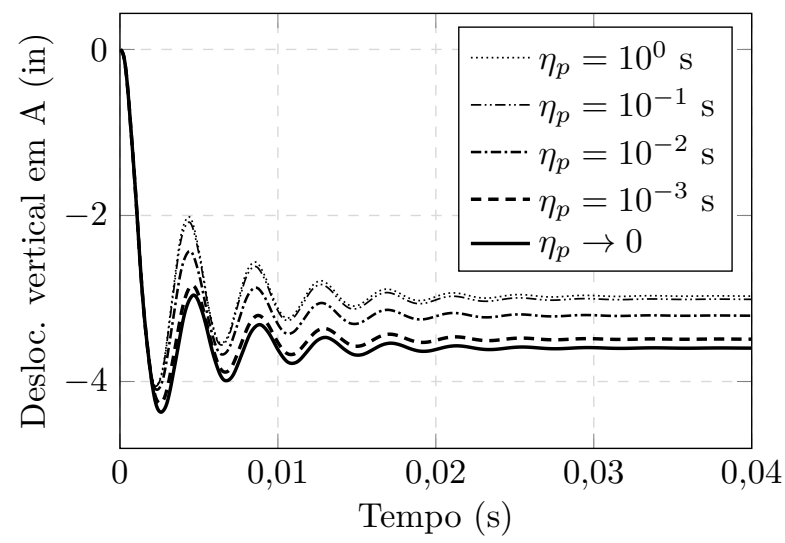

(b) Diferença entre deslocamentos finais

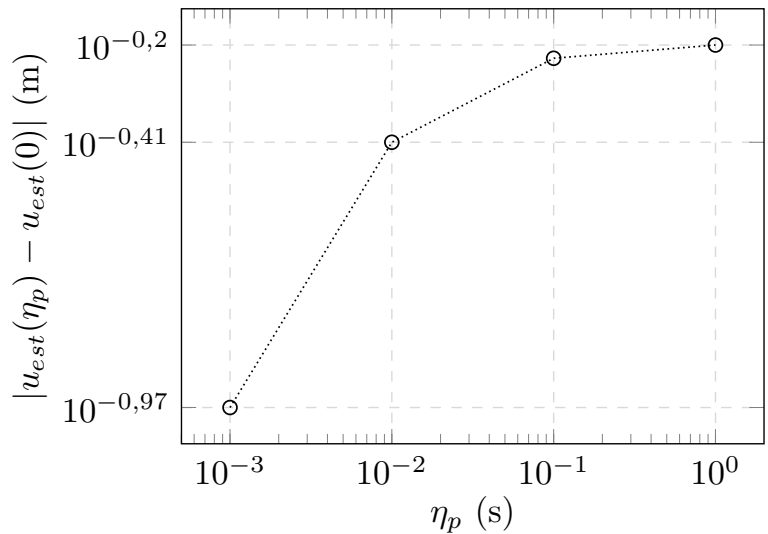

Na Figura 76 são apresentadas as configurações deformadas estacionárias da viga para alguns valores de $\eta_{p}$ considerados. Além disso, na Figura 77, apresentam-se os diagramas de tensões na seção engastada da viga para esses casos, traçados com base nos 
valores nodais. Nesses diagramas, observa-se uma maior influência da plasticidade para menores valores de $\eta_{p}$.

Figura 76 - Configurações finais de viga visco-elasto-plástica para $\eta=8$ s e diferentes valores de $\eta_{p}$, apresentando componentes $\sigma_{11}$ da tensão de Cauchy em mapas de cores

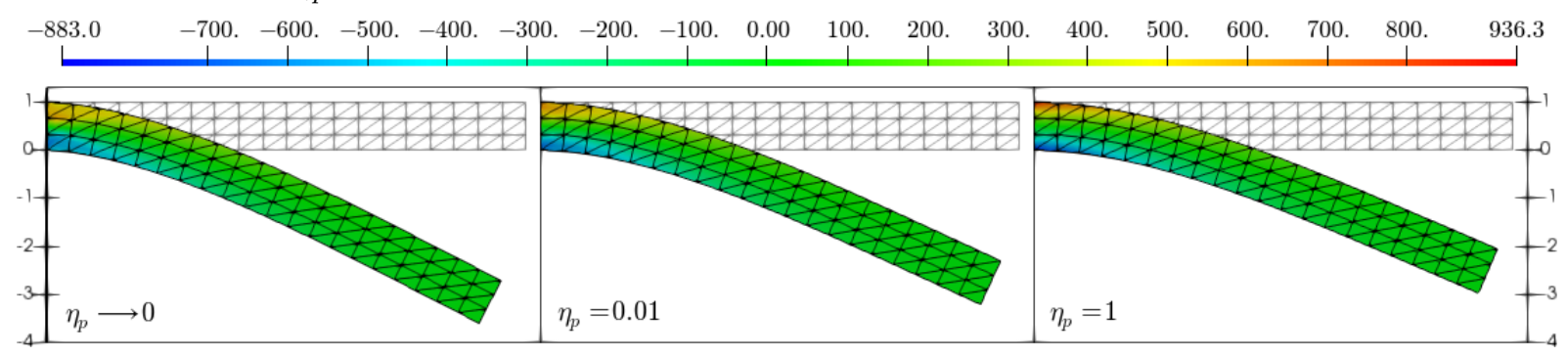

Figura 77 - Diagramas de tensão na seção engastada da viga após estabilização para modelo visco-elasto-plástico com $\eta=8 \mathrm{~s}$ e diferentes valores de $\eta_{p}$

(a) $\eta_{p} \rightarrow 0$

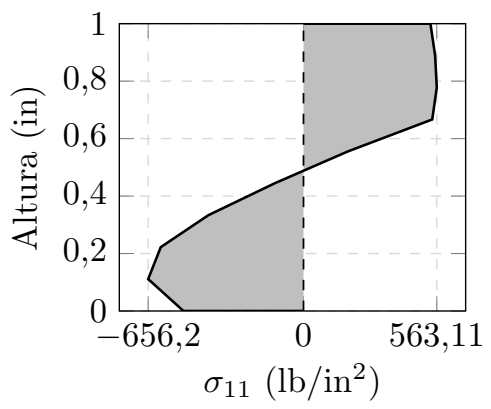

(b) $\eta_{p}=0,01 \mathrm{~s}$

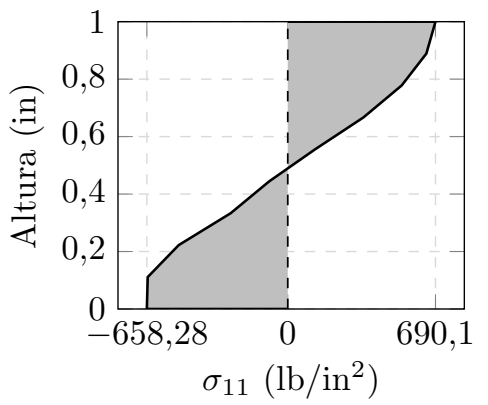

(c) $\eta_{p}=1 \mathrm{~s}$

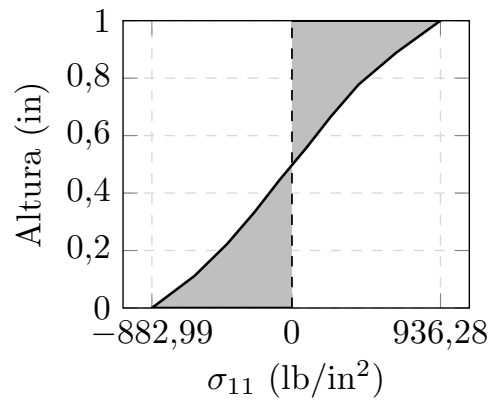

\subsection{Dobramento simples de chapa metálica}

Considera-se neste exemplo uma chapa metálica, inicialmente retangular com dimensões $L \times H$, forçada a assumir uma forma curva quando pressionada entre dois blocos rígidos (ver Figura 78). Adota-se o modelo elasto-plástico, com parâmetros do material Mild Steel Ck15 dados na Tabela 2, e estado plano de deformações. Os corpos rígidos neste trabalho são modelados por meio de restrições de deslocamento aplicadas ao longo de todo o domínio.

Figura 78 - Geometria e malha para exemplo de dobramento simples em chapa metálica
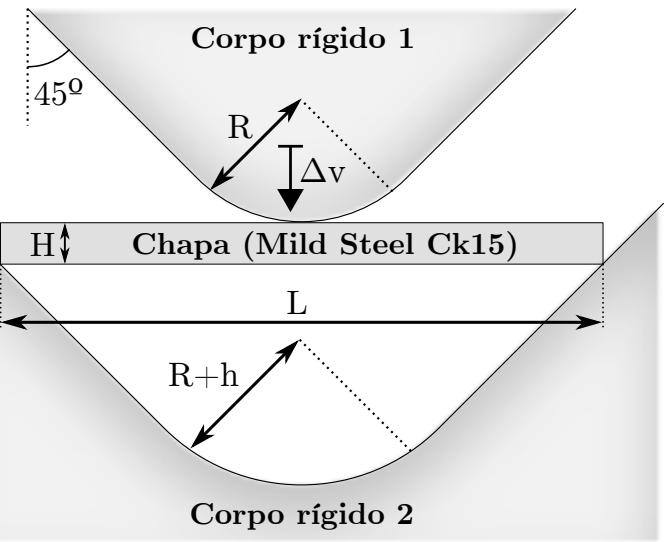

\section{Geometria:}

$\mathrm{L}=1.0 \mathrm{~m}$

$\mathrm{H}=0.025 \mathrm{~m}$

$\mathrm{R}=0.25 \mathrm{~m}$

$\Delta \mathrm{v}=0.38609 \mathrm{~m}$

\section{Malha:}

Corpo rígido 1: 461 nós, 94 elementos triangulares Corpo rígido 2: 389 nós, 78 elementos triangulares Chapa: 1267 nós, 254 elementos triangulares

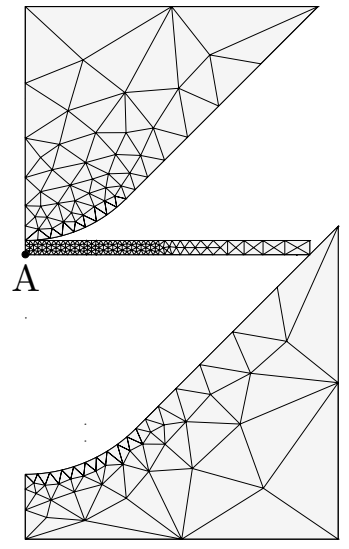


Aplica-se deslocamento prescrito no bloco superior de forma que entre ele e o bloco inferior resulte apenas um espaço $H$, suficiente para que a barra seja forçada ao formato desejado. Tal processo é realizado em uma análise estática de 3000 passos. No passo seguinte, o bloco superior é afastado de forma que possa ser observado o recuo (Springback) da chapa.

O contato é resolvido pela estratégia nó-a-segmento, sendo as interfaces dos anteparos rígidos tratadas como segmentos alvos, e as da chapa como nós projéteis. Devido à simetria, apenas metade do problema é discretizado, sendo aplicadas restrições de deslocamentos horizontais nos contornos pertencentes ao eixo de simetria. A fim de reduzir o custo computacional, refina-se a malha apenas na região central da chapa, onde há maiores níveis de deformações.

Na Figura 79 apresenta-se a configuração deformada do problema para determinados passos, bem como a deformação plástica de Green-Lagrange na direção horizontal. De forma a medir o Springback, calculou-se o ângulo final entre as duas extremidades e o recuo vertical do ponto A, mostrados na Figura 82a.

Figura 79 - Configuração deformada para o exemplo de dobramento simples, com valores de $E_{11}^{p}$ apresentados em mapa de cores

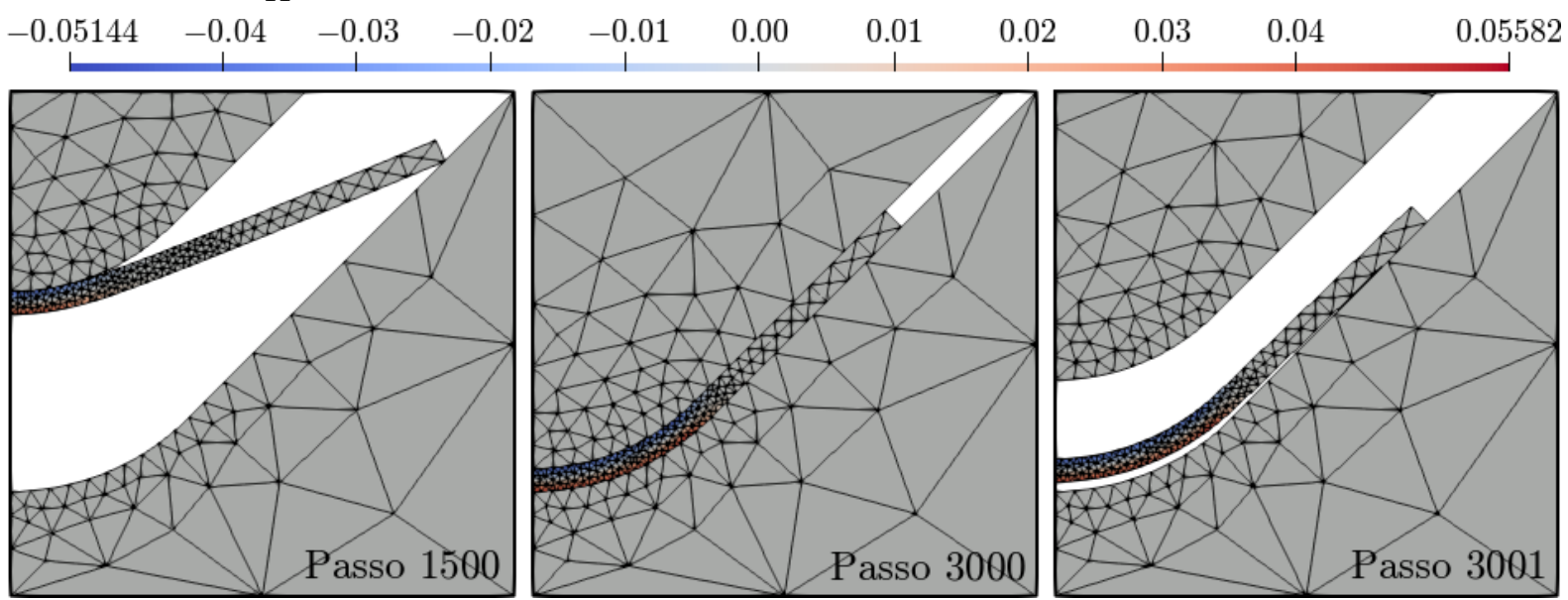

\subsection{Dobramento direcionado (Draw Bending) de chapa metálica}

Nesta seção apresenta-se um procedimento alternativo ao do exemplo anterior para o dobramento de chapa metálica, demonstrando como a diferença no processo de manufatura pode alterar o comportamento do Springback. Novamente, adota-se uma chapa metálica com material elasto-plástico (Mild Steel Ck15) de dimensões $L \times H$ e estado plano de deformações, porém, de forma a suavizar o contato, considera-se um formato semicircular na extremidade direita. Inicialmente, dois blocos rígidos posicionados conforme a Figura 80 são movidos em direção à chapa enquanto a mesma tem sua extremidade esquerda totalmente restrita, forçando sua conformação. Nessa etapa, adota-se uma análise estática com 3000 passos. No passo seguinte, os blocos são afastados de forma que possa ser observado o Springback. 
Figura 80 - Geometria e malha para exemplo de dobramento direcionado em chapa metálica
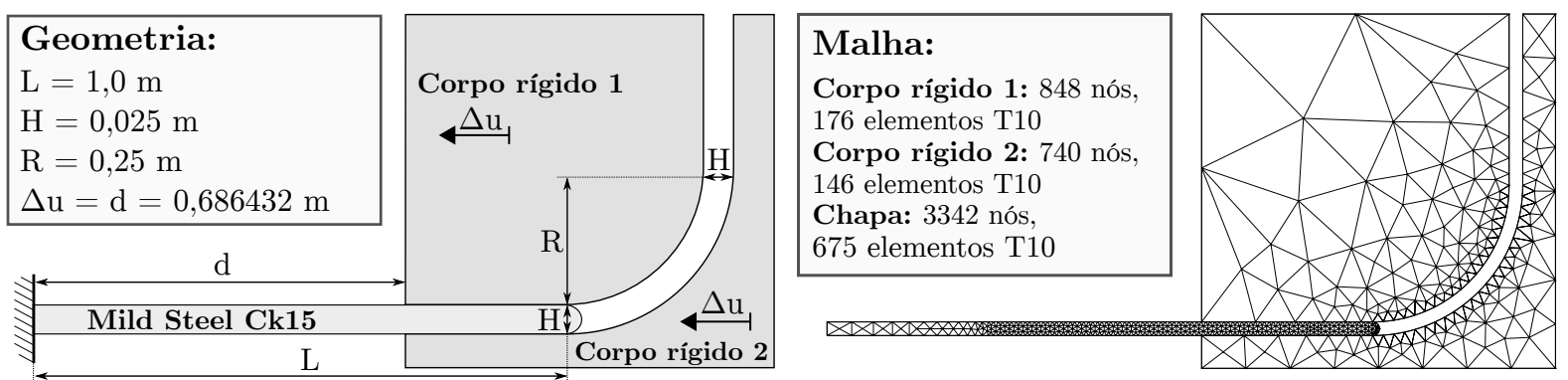

A principal diferença entre o dobramento direcionado apresentado neste exemplo e o dobramento simples do exemplo anterior é que neste algumas regiões da chapa são sujeitas a um comportamento cíclico de tensões e deformações, devido ao fato de as seções da extremidade direita serem inicialmente dobradas e em seguida forçadas novamente à configuração reta, provocando o efeito de Bauschinger. Na Figura 81 são apresentadas as configurações deformadas para alguns passos, onde nota-se que os valores de deformação de Green-Lagrange são bem próximos aos obtidos no exemplo anterior (Figura 79). Entretanto, observam-se pequenas deformações residuais na região superior da chapa, que influenciam no comportamento do Springback: o formato final obtido demonstra uma melhor proximidade ao formato imposto quando comparado com o do exemplo anterior, e, mais ainda, apresenta uma curvatura na direção contrária à obtida anteriormente, formando um ângulo agudo entre as extremidades, como pode ser visto na Figura 82b.

Figura 81 - Configuração deformada para o exemplo de dobramento direcionado, com valores de $E_{11}^{p}$ apresentados em mapa de cores

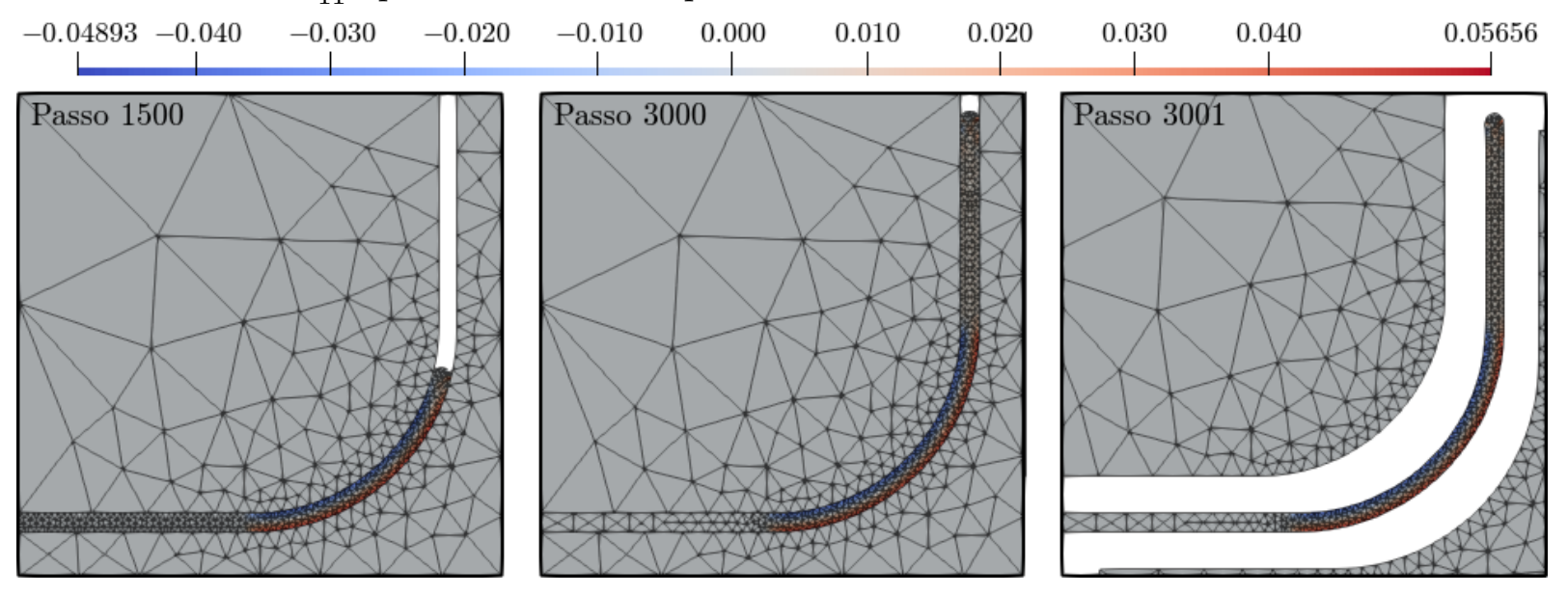

Figura 82 - Springback manifestado nos exemplos de dobramento

(a) Dobramento simples

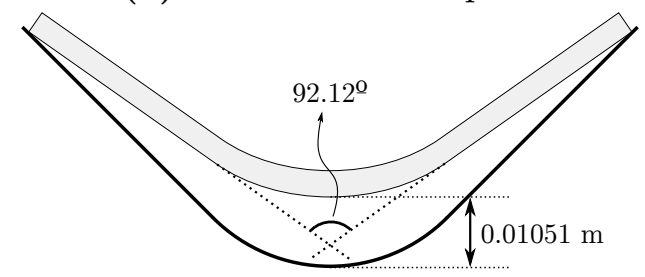

(b) Dobramento direcionado

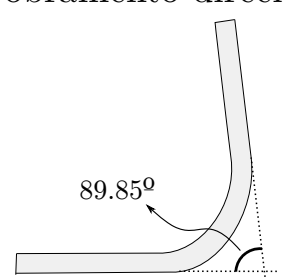




\subsection{Extrusão}

Neste exemplo, uma chapa com formato representado na Figura 83 é compelida contra uma abertura de altura mínima igual a $60 \%$ da sua altura inicial, com a intenção de provocar uma redução na seção transversal e, como consequência, um aumento de seu comprimento. O maquinário é composto por dois setores circulares, tratados como anteparos rígidos. Esses são movidos em direção à chapa por meio de deslocamento prescrito em uma análise estática. Novamente aproveita-se a simetria do problema para reduzir pela metade o domínio efetivamente discretizado.

Figura 83 - Geometria e malha para exemplo de extrusão

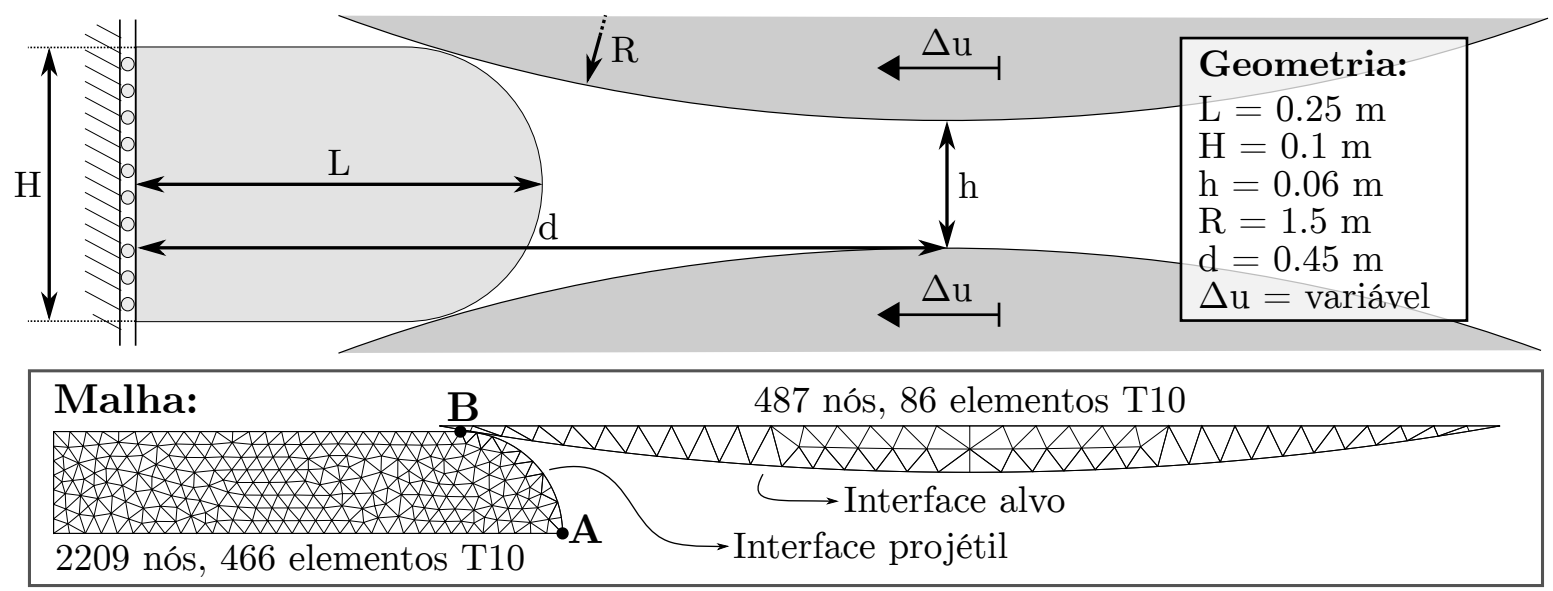

\subsubsection{Material metálico com modelo elasto-plástico}

Neste caso, considerou-se o material elasto-plástico Mild Steel Ck15, cujos parâmetros são dados na Tabela 2, e deslocamento prescrito total $\Delta u=0,45 \mathrm{~m}$, aplicado em 8000 passos. Apresenta-se na Figura 84a o deslocamento horizontal no ponto da extremidade direita da chapa (ponto A), e na Figura 84b o deslocamento vertical no ponto que inicia o trecho circular da chapa (ponto B).

Figura 84 - Gráficos de deslocamento para o exemplo de extrusão em material metálico

(a) Deslocamento horizontal em A

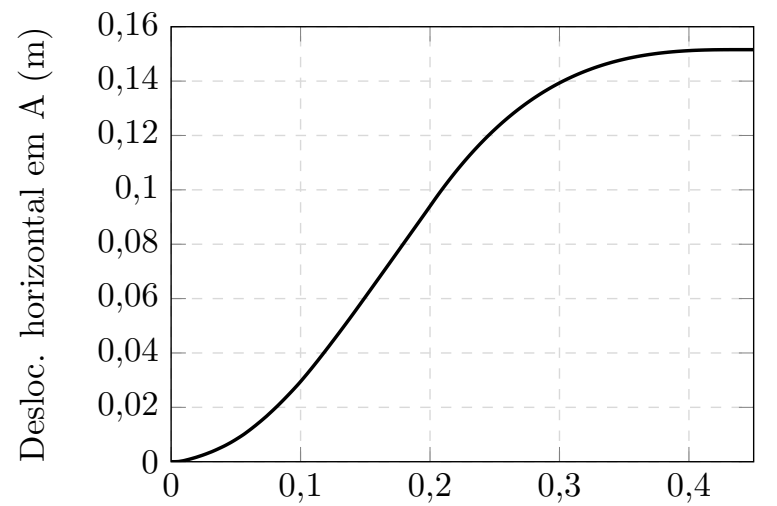

Desloc. do anteparo rígido em módulo (m) (b) Deslocamento vertical em B

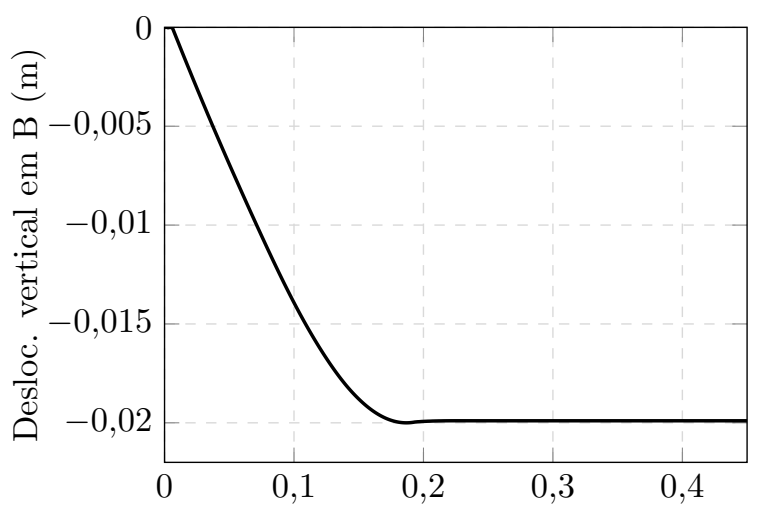

Desloc. do anteparo rígido em módulo $(\mathrm{m})$ 
Conforme já observado nos exemplos anteriores, o material metálico em grandes deformações possui parcelas elásticas muito pequenas quando comparadas às plásticas, e, portanto, se conforma mais facilmente aos formatos impostos quando comparado ao material polimérico. De fato, com base nos dados da Figura 84b, verifica-se que a altura final da chapa na seção do ponto B é $0,06025898 \mathrm{~m}$, isto é, houve um aumento de apenas $2,5898 \cdot 10^{-4} \mathrm{~m}$ com relação à altura de $0,06 \mathrm{~m}$ imposta pelos anteparos rígidos. Nas demais seções essa tendência é mantida, como pode ser observado nas configurações deformadas da chapa (ver Figura 85).

Figura 85 - Configurações deformadas para exemplo de extrusão em chapa metálica elastoplástica, valores de $E_{11}^{p}$ apresentados em mapas de cores
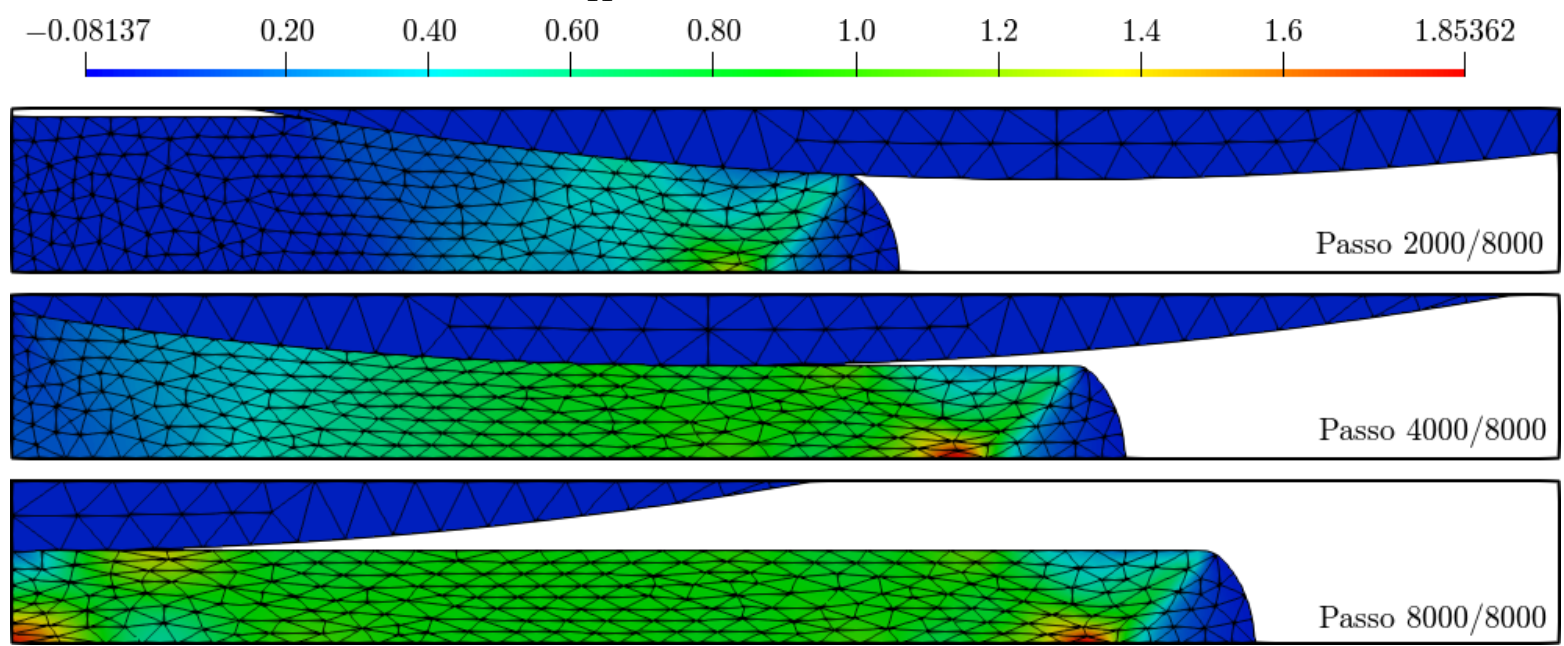

\subsubsection{Material polimérico com modelo visco-elasto-plástico}

Neste caso, considerou-se o material visco-elasto-plástico da Tabela 9, e deslocamento prescrito total $\Delta u=1,8 \mathrm{~m}$, aplicado de forma incremental ao longo de 30000 passos, com tempo máximo $\left(t_{\max }\right)$ variável. Na Figura 86 são apresentados os deslocamentos nos pontos A e B para três diferentes casos.

Figura 86 - Gráficos de deslocamento para o exemplo de extrusão em material polimérico

(a) Deslocamento horizontal em A

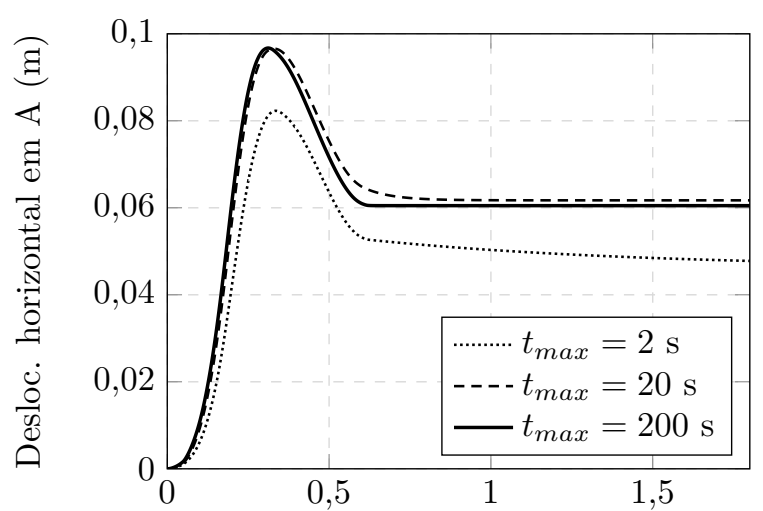

Desloc. do anteparo rígido em módulo (m) (b) Deslocamento vertical em B

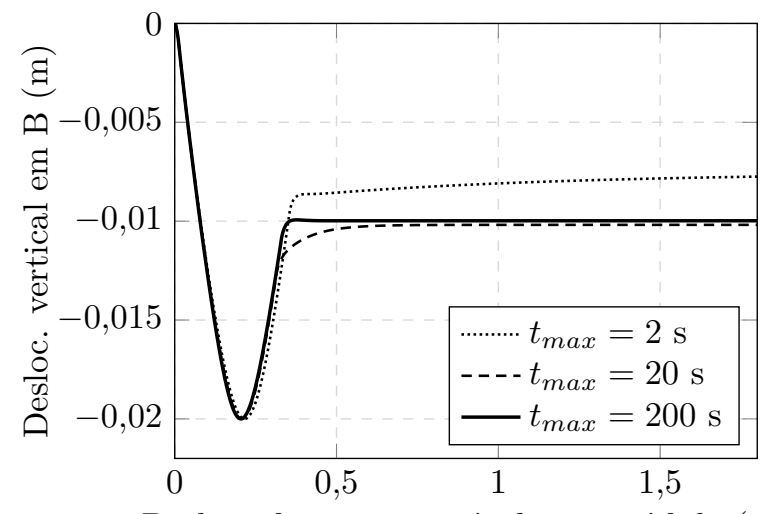

Desloc. do anteparo rígido em módulo (m) 
Comparado ao material metálico, este apresenta parcelas de deformações elásticas significativas, o que resulta em uma curva de retorno mais acentuada, como pode ser visto nos gráficos da Figura 86. Dentre os casos analisados, o maior recuo elástico é observado no caso intermediário $t_{\max }=20 \mathrm{~s}$, onde obtem-se um comprimento final (medido no ponto A) de 0,311716 m, e altura final (medida na seção do ponto B) de 0,0796267 m, que equivale à quase $80 \%$ da altura inicial. A maior altura final é observada no caso $t_{\max }=2 \mathrm{~s}$, onde, embora o tempo de análise não tenha sido suficiente para que as respostas se estabilizassem satisfatoriamente, obteve-se altura final de quase $85 \%$ da inicial $(0,0845064 \mathrm{~m})$. As configurações deformadas para esse caso são apresentadas na Figura 87.

Figura 87 - Configurações deformadas para exemplo de extrusão em chapa polimérica no caso $t_{\text {max }}=2 \mathrm{~s}$, com valores de $E_{11}^{p}$ apresentados em mapas de cores
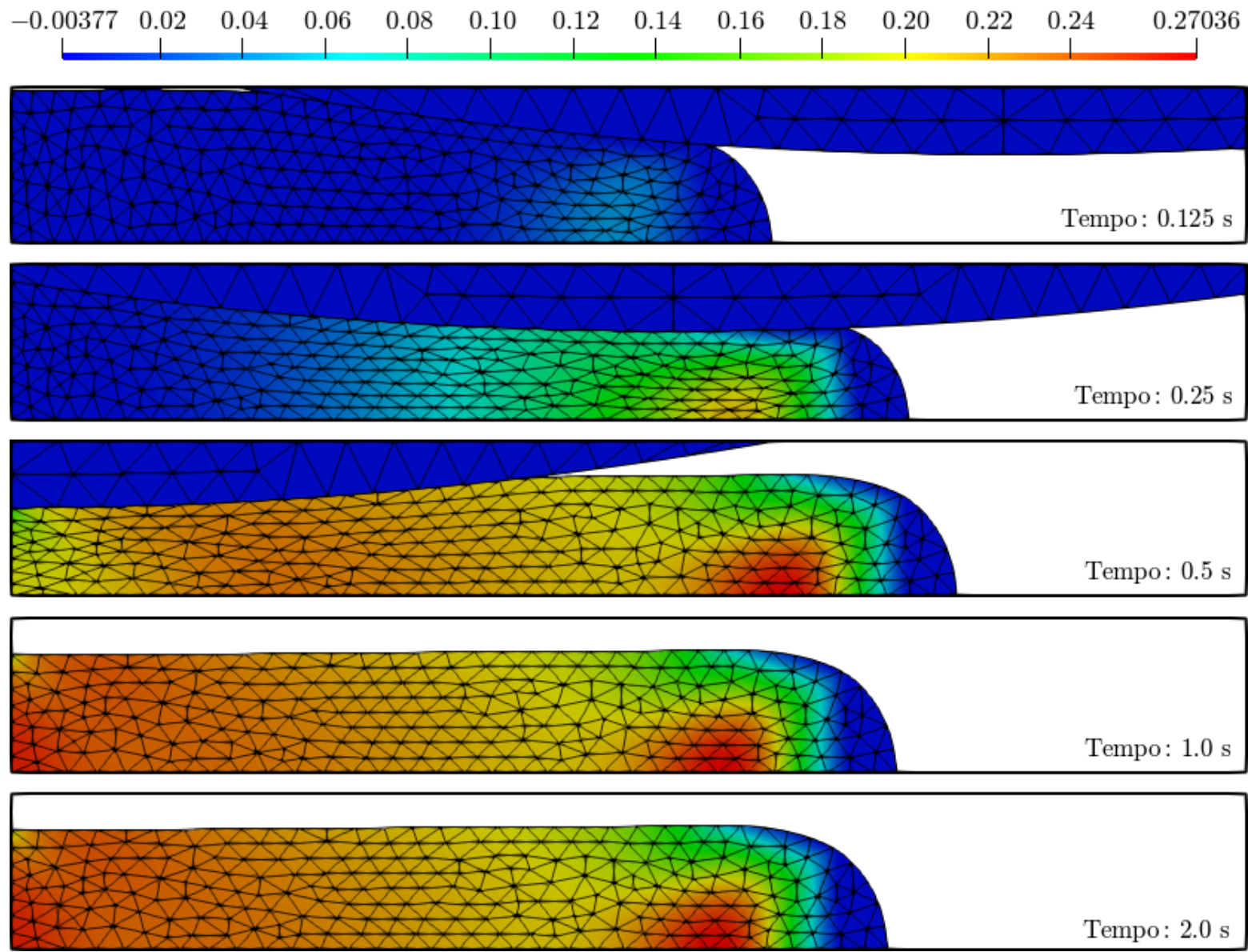

\subsection{Impacto entre corpos deformáveis}

Considera-se neste exemplo o problema dinâmico de impacto entre dois sólidos com formatos circular e retangular, respectivamente. A geometria e malha são apresentadas na Figura 88, onde, devido à simetria, apenas metade do problema é discretizado. Considerouse estado plano de tensões, e massa $\rho=0,25 \mathrm{~kg} / \mathrm{m}^{3}$ para ambos os sólidos, sendo que apenas no sólido circular considera-se o peso próprio do corpo. A aceleração da gravidade adotada nesse caso é $g=10 \mathrm{~m} / \mathrm{s}^{2}$, o que implica em um peso resultante de $-2,5 \mathrm{~N} / \mathrm{m}^{2}$. 
Figura 88 - Geometria e malha para exemplo de impacto entre corpos deformáveis
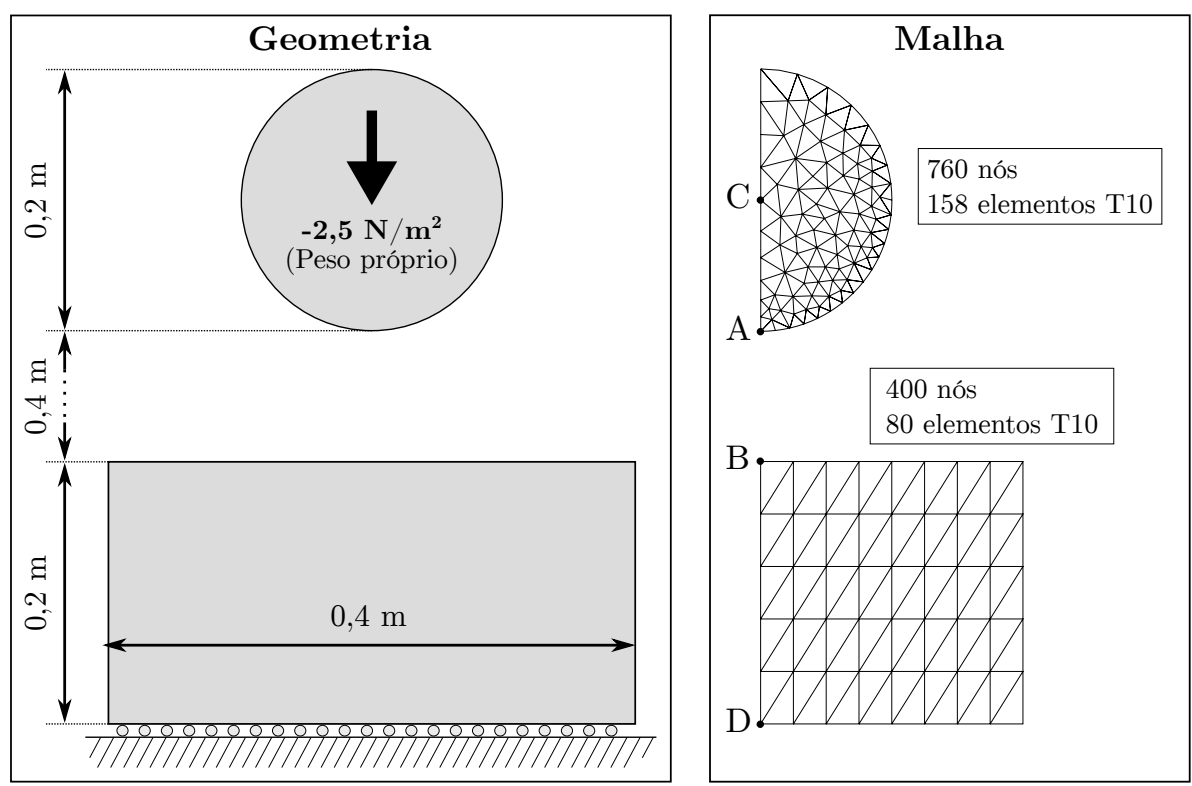

É importante ser observado que problemas de impacto enfrentam dificuldades de convergência quando utilizado o método de Newmark- $\beta$ com os parâmetros tradicionais $(\beta=1 / 4$ e $\gamma=1 / 2)$. Esse problema pode ser contornado com o aumento do valor de $\Delta t$, uma vez que isso impede o método numérico de capturar campos oscilatórios de alta frequência, os quais impedem a convergência. Tal artifício, entretanto, pode comprometer os resultados obtidos, especialmente em problemas que dependam de uma boa discretização temporal. Dessa forma, Hu (1997) propôs um método de Newmark modificado utilizando os parâmetros $\beta=1$ e $\gamma=3 / 2$, que se mostrou bastante eficiente para tratar problemas de impacto. Tal modificação, entretanto, deve ser utilizada com cautela, pois introduz na integração temporal um amortecimento numérico altamente sensível à discretização de tempo adotada. Em geral, é necessário que sejam utilizados valores de $\Delta t$ muito menores que os habituais para diminuir as discrepâncias causadas por tal fenômeno dissipativo.

\subsubsection{Modelo hiperelástico}

Este primeiro caso é utilizado para comparar as respostas dos dois integradores temporais considerados, e observar a eficiência do método de $\mathrm{Hu}$ (1997) quando utilizados valores de $\Delta t$ suficientemente pequenos. Considera-se um modelo hiperelástico neo-Hookeano em ambos os sólidos com $\Lambda=200 \mathrm{~N} / \mathrm{m}^{2}$ e $\mu=15 \mathrm{~N} / \mathrm{m}^{2}$. O tempo máximo de análise neste caso é $t_{\max }=0,65625 \mathrm{~s}$, discretizado, para o integrador temporal de $\mathrm{Hu}$, em seis números de passos diferentes: 3500, 7000, 14000, 28 000, 56000 e 112000 . Para o método de Newmark- $\beta \operatorname{com} \beta=1 / 4$ e $\gamma=1 / 2$, apenas foi possível obter convergência com 1000 passos de tempo, isto é, $\Delta t=0,65625 \cdot 10^{-3} \mathrm{~s}$.

Na Figura 89 são mostrados os gráficos de deslocamento ao longo do tempo nos pontos A (nó inferior do sólido circular) e B (nó superior do sólido retangular). Devido ao efeito de amortecimento numérico previamente mencionado, as amplitudes no integrador 
de Hu tendem a diminuir à medida que o tempo avança, causando variações indesejáveis entre as discretizações temporais adotadas. Essa variação é notável, por exemplo, entre os casos com 3500 e 14000 passos, porém, esse último já se mostra bem próximo do resultado obtido no método de Newmark- $\beta$ com os parâmetros tradicionais. Pode-se presumir, portanto, que a resposta tende a convergir para números de passo suficientemente grandes. A fim de investigar essa convergência, apresentam-se nos gráficos da Figura 90 os erros em deslocamento relativos ao Newmark $\operatorname{com} \beta=1 / 4$ e $\gamma=1 / 2$ no instante $t=0,65625 \mathrm{~s}$, para cada $\Delta t$ adotado. As inclinações médias (ordens de convergência) das retas são de 0,63989429 e 0,690037326, respectivamente, sendo observado em ambas uma leve redução nos casos mais refinados. Para esses casos, o erro pode ser desprezado em aplicações práticas, mostrando que o integrador de $\mathrm{Hu}$ fornece resultados satisfatórios sem a necessidade de limitar o refinamento adotado para a discretização temporal.

Figura 89 - Gráficos de deslocamento para o exemplo de impacto com modelo hiperelástico

(a) Deslocamento vertical em A

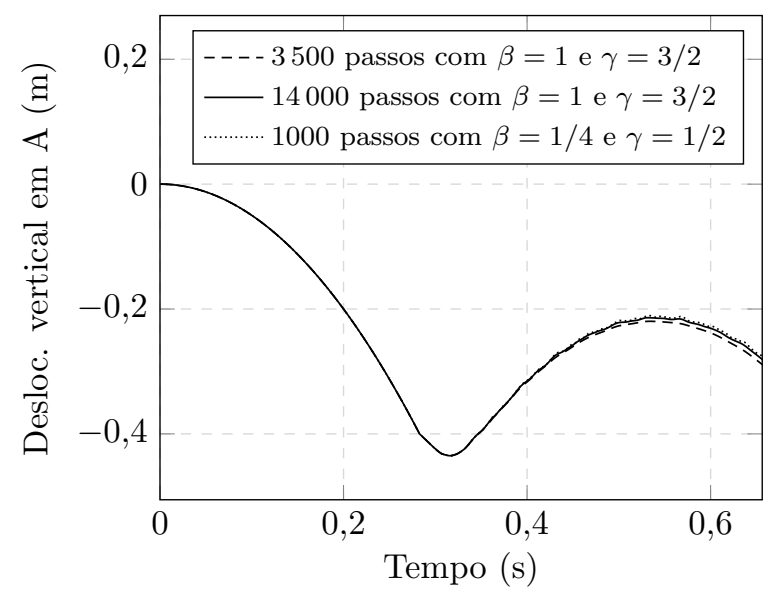

(b) Deslocamento vertical em B

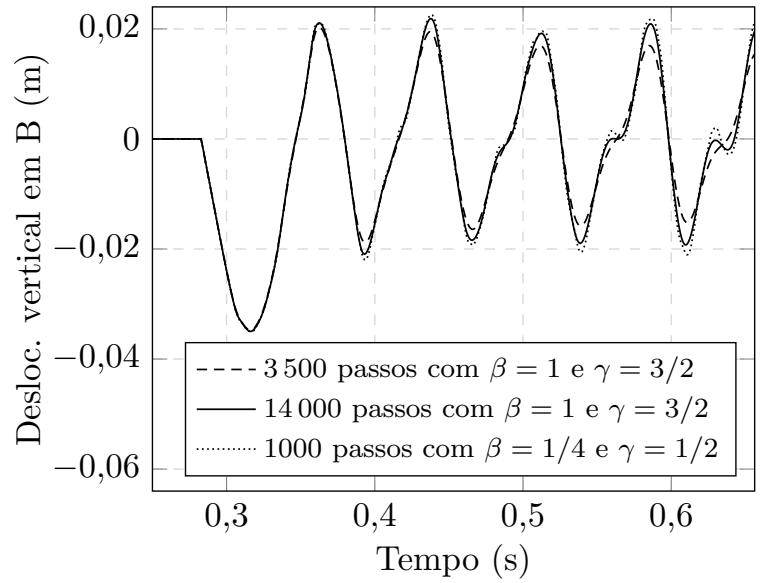

Figura 90 - Análise de convergência para deslocamentos finais no exemplo de impacto com modelo hiperelástico, em escala logarítmica na base 2

(a) Ponto A

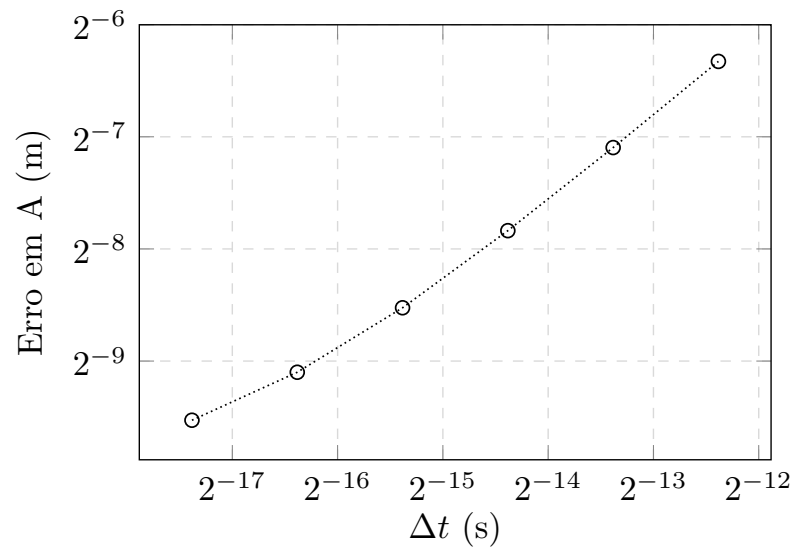

(b) Ponto B

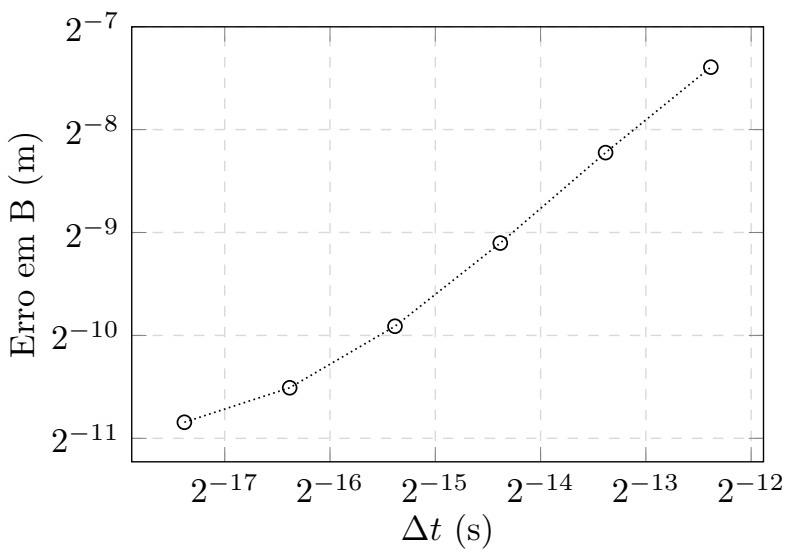

Na Figura 91 são mostrados os gráficos da componente $\sigma_{22}$ de tensão de Cauchy ao longo do tempo para ambos os integradores, medido no ponto C (centro do sólido circular). 
Mesmo com apenas 1000 passos de tempo, pode-se observar que o método de Newmark- $\beta$ $\operatorname{com} \beta=1 / 4$ e $\gamma=1 / 2$ captura um número maior de frequências quando comparado com o de $\mathrm{Hu}$, sendo essas mesmas frequências responsáveis pela instabilidade numérica que, neste caso, impediu a convergência quando adotado 2000 passos ou mais. Na Figura 92 são mostradas as configurações deformadas em determinados passos de tempo para o caso com integrador temporal de $\mathrm{Hu}$ e 112000 passos.

Figura 91 - Tensão de Cauchy $\left(\sigma_{22}\right)$ no ponto C para impacto com modelo hiperelástico

(a) $\beta=1$ e $\gamma=3 / 2$

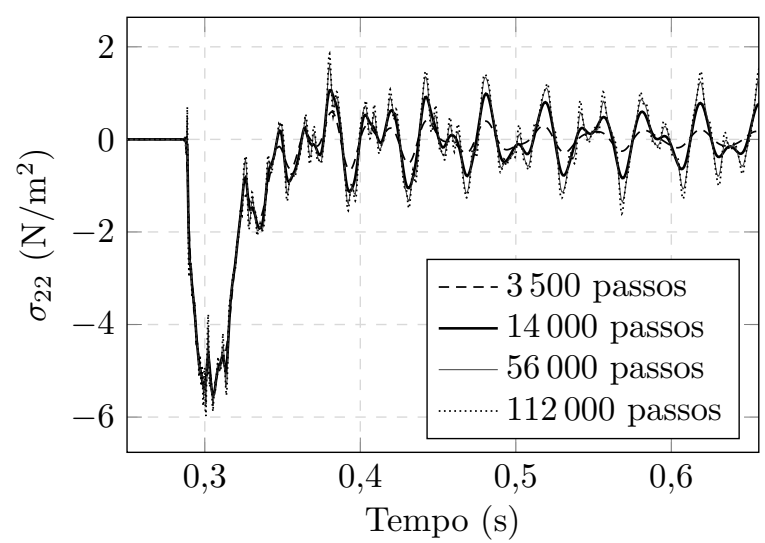

(b) $\beta=1 / 4$ e $\gamma=1 / 2$

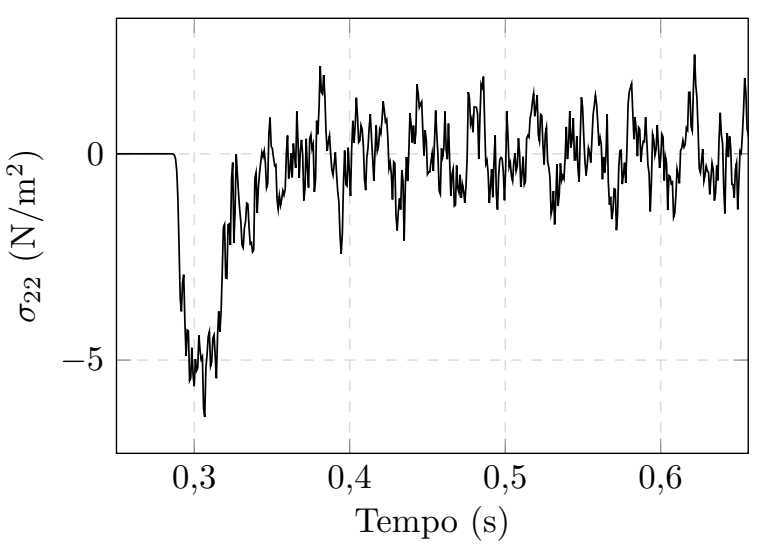

Figura 92 - Configurações deformadas de problema de impacto com 112000 passos, mostrando a componente $\sigma_{22}$ das tensões de Cauchy em mapas de cores

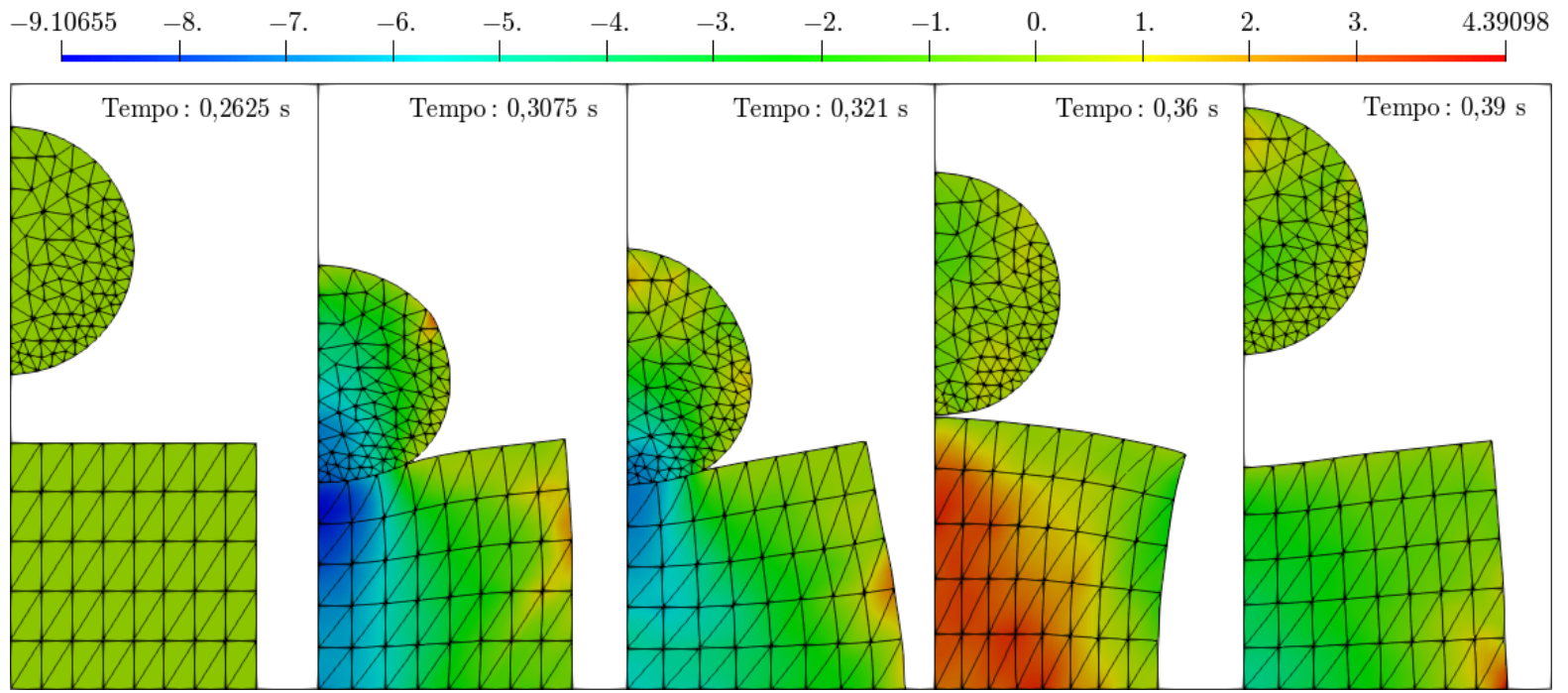

\subsubsection{Modelo visco-elasto-plástico}

Embora no caso anterior tenha sido viável utilizar o integrador temporal de Newmark- $\beta$ com os parâmetros tradicionais limitando o número de passos adotado, no caso visco-elasto-plástico isso não foi possível, devido à alta dependência do modelo sobre o refinamento da discretização temporal. De fato, a convergência é prejudicada para valores muito grandes de $\Delta t$, uma vez que até mesmo o operador tangente consistente do modelo depende da discretização (como pode ser observado na seção 6.5). Por outro lado, valores 
muito pequenos de $\Delta t$ provocam, em problemas de impacto, a instabilidade numérica já abordada anteriormente. Dessa forma, considera-se apenas o integrador de $\mathrm{Hu}$ neste caso, com uma discretização temporal suficientemente refinada.

O material adotado em ambos os sólidos possui as propriedades dadas na Tabela 15. Neste caso, considerou-se o tempo máximo $t_{\max }=3,5 \mathrm{~s}$, discretizado em 350000 passos (isto é, $\Delta t=10^{-5} \mathrm{~s}$ ).

Tabela 15 - Parâmetros de material visco-elasto-plástico para exemplo de impacto

\begin{tabular}{|c|c|c|c|c|}
\hline \multicolumn{5}{|c|}{ Parâmetros visco-elásticos } \\
\hline$\Lambda^{\infty}\left(\mathrm{N} / \mathrm{m}^{2}\right)$ & $\mu^{\infty}(\mathrm{N} /$ & $\Lambda^{e}\left(\mathrm{~N} / \mathrm{m}^{2}\right)$ & $\mu^{e}\left(\mathrm{~N} / \mathrm{m}^{2}\right)$ & $\eta\left(\mathrm{s} \cdot \mathrm{N} / \mathrm{m}^{2}\right)$ \\
\hline 10 & 2 & 10 & 4 & 1 \\
\hline \multicolumn{2}{|c|}{ Parâmetros de encruamento } & \multicolumn{3}{|c|}{ Parâmetros de Perzyna/Norton } \\
\hline$\sigma_{Y}\left(\mathrm{~N} / \mathrm{m}^{2}\right)$ & $c\left(\mathrm{~N} / \mathrm{m}^{2}\right)$ & $\eta_{p}(\mathrm{~s})$ & $\alpha_{p}\left(\mathrm{~N} / \mathrm{m}^{2}\right)$ & $m$ \\
\hline 0.4 & 1,5 & 2 & 0.4 & 1 \\
\hline
\end{tabular}

Os gráficos de deslocamento vertical, componente de tensão $\sigma_{22}$ e componente de deformação plástica $E_{22}^{p}$ ao longo do tempo são mostrados nas Figuras 93, 94 e 95, respectivamente. O primeiro é medido novamente nos pontos A e B. Já os dois últimos são medidos nos pontos C e D (ver Figura 88). Como esperado, as respostas tendem a se estabilizar, devido ao efeito dissipativo do modelo constitutivo. Na Figura 96 são mostradas as configurações deformadas para determinados instantes, incluindo a configuração "estabilizada".

Figura 93 - Deslocamentos para o exemplo de impacto com modelo visco-elasto-plástico

(a) Deslocamento vertical em A

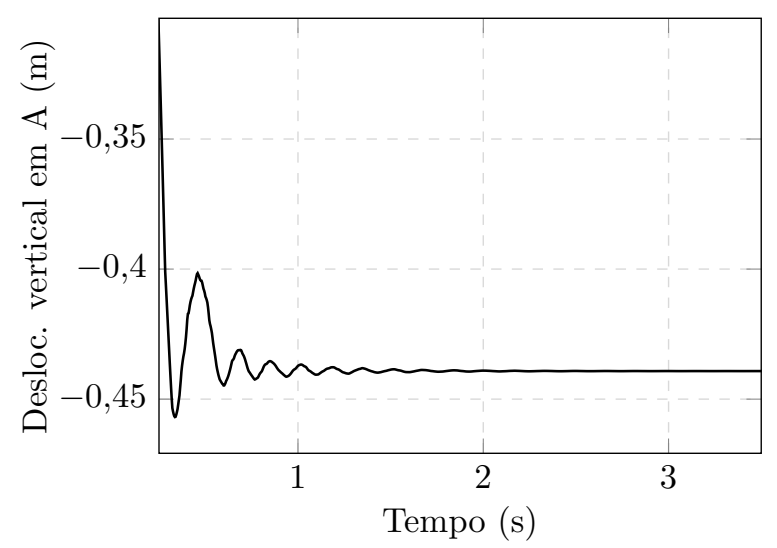

(b) Deslocamento vertical em B

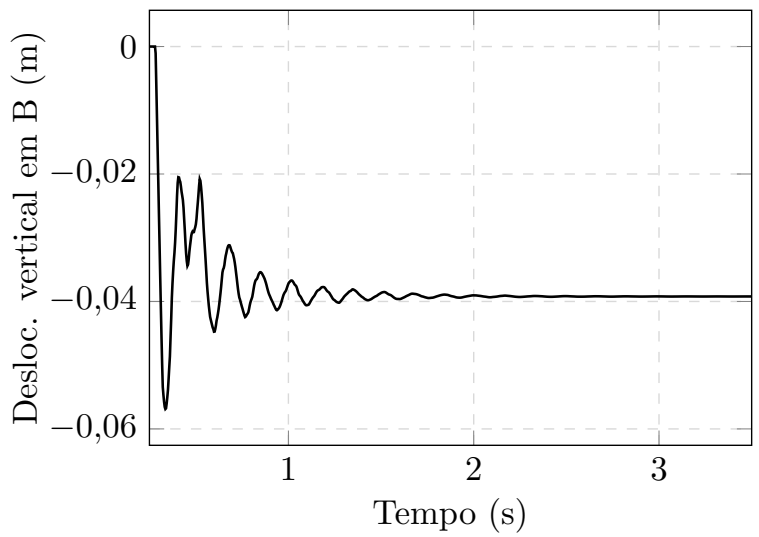


Figura 94 - Tensão de Cauchy para o exemplo de impacto com modelo visco-elasto-plástico

(a) Valores de $\sigma_{22}$ no ponto $\mathrm{C}$

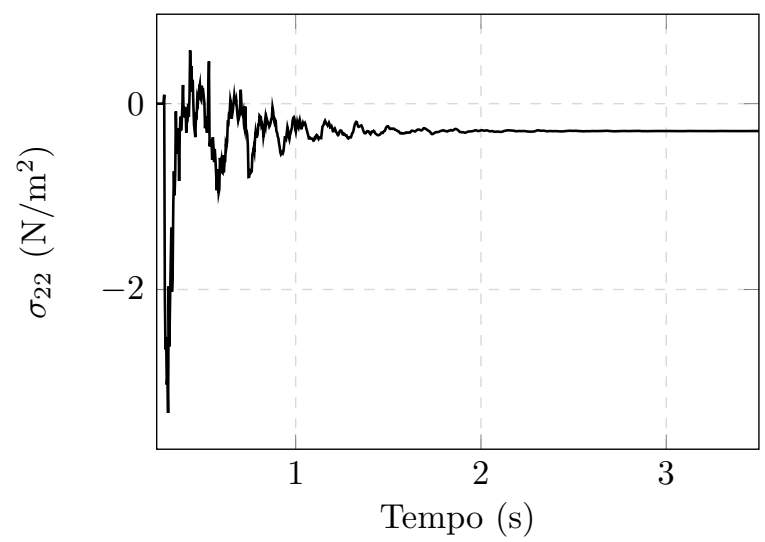

(b) Valores de $\sigma_{22}$ no ponto $\mathrm{D}$

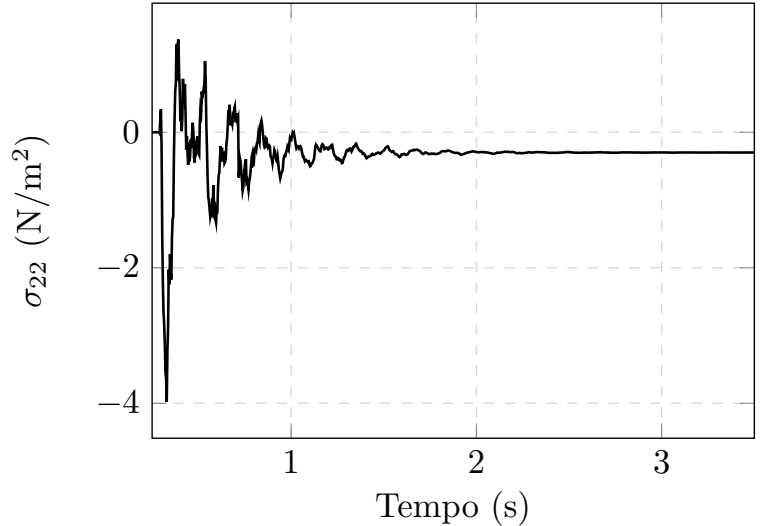

Figura 95 - Deformações plásticas para o exemplo de impacto com modelo visco-elasto-plástico

(a) Valores de $E_{22}^{p}$ no ponto C

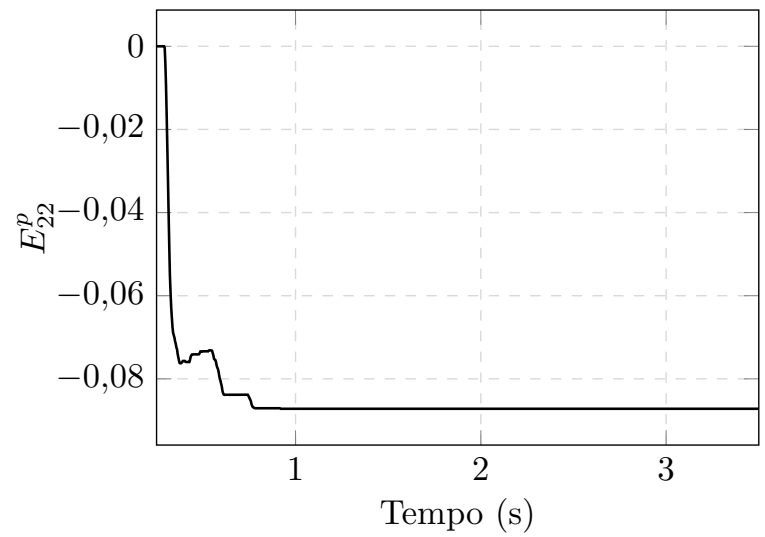

(b) Valores de $E_{22}^{p}$ no ponto D

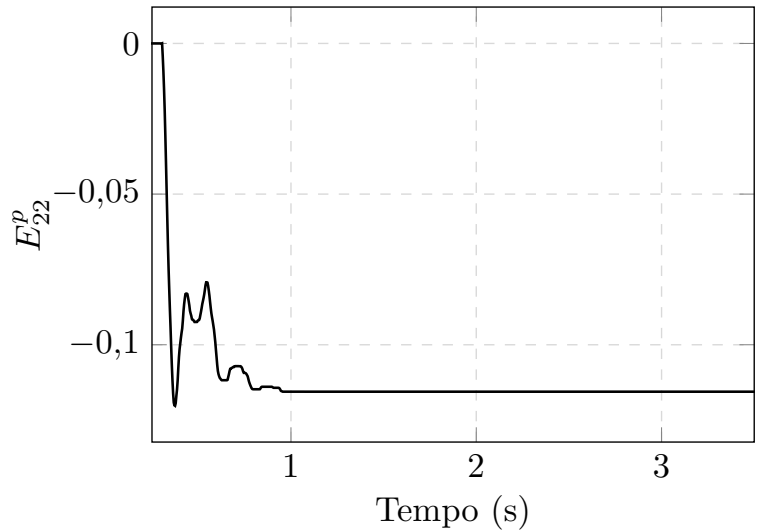

Figura 96 - Configurações deformadas de problema de impacto com material visco-elastoplástico, apresentando valores de $E_{22}^{p}$ em mapas de cores

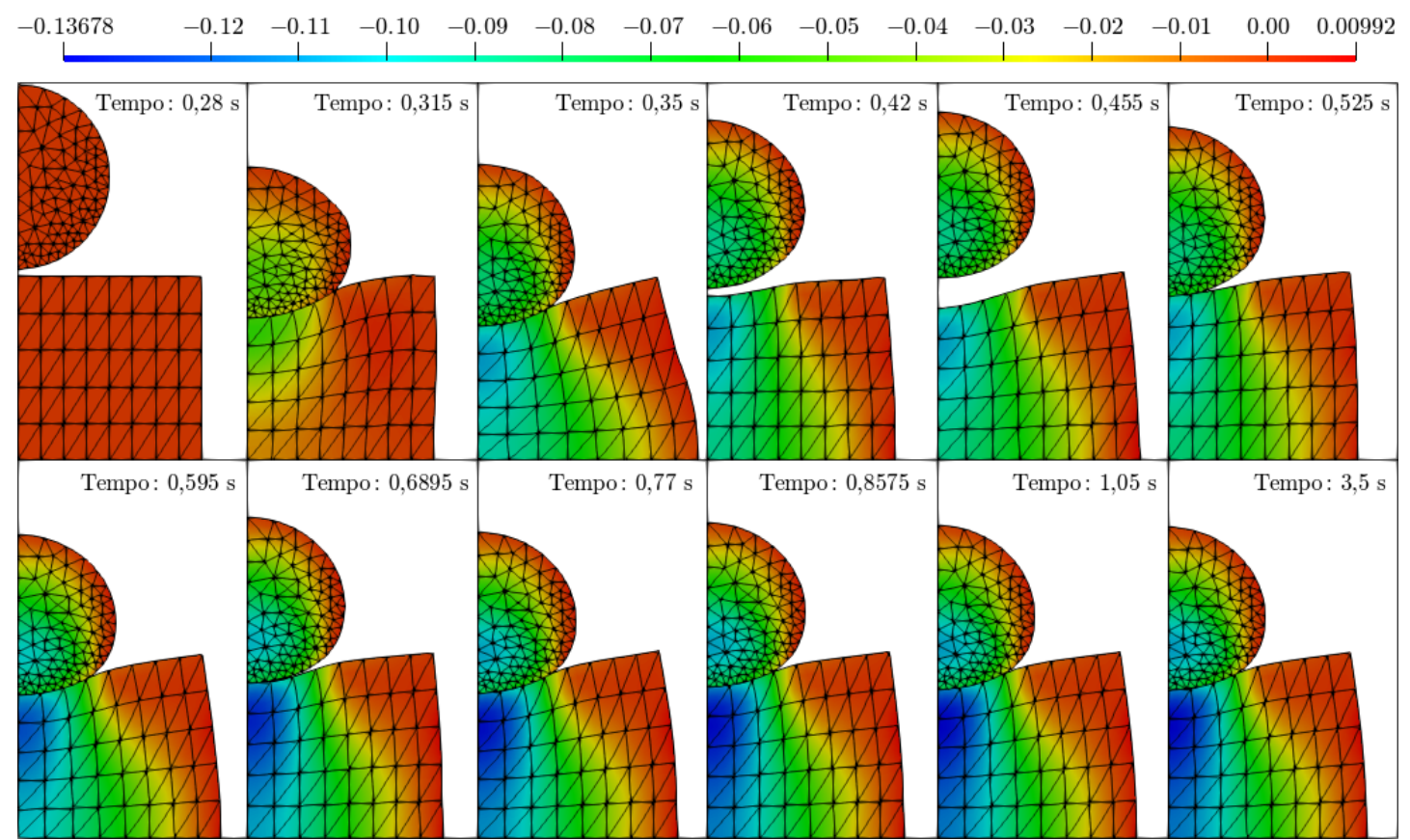




\subsection{Abatimento em material altamente viscoso}

O ensaio de abatimento de tronco de cone (slump test) é um procedimento realizado para medir a consistência do concreto em estado fresco. Nesse ensaio, o material é depositado em um molde com formato cônico, e em seguida liberado, de forma que se deforme sobre a ação do próprio peso. Não é o escopo deste exemplo simular um ensaio de abatimento real, devido a diversos fatores como: necessidade de uma análise tridimensional, e da consideração de atrito entre o sólido e o chão, alta complexidade do concreto em estado fresco, entre outros. Entretanto, para mostrar as possibilidades do código desenvolvido, apresenta-se um exemplo inspirado por esse processo.

Considera-se um sólido com formato trapezoidal e malha conforme a Figura 97 (onde foi aproveitada a simetria do problema). Adota-se um modelo constitutivo viscoelasto-plástico, com parâmetros dados na Tabela 16. Embora não tenha sido utilizado um material semelhante ao concreto fresco, esse foi definido de forma que apresentasse comportamento altamente viscoso, isto é, taxas de deformação relativamente baixas para os esforços considerados. Foi adotado estado plano de tensões, massa específica $\rho=0,15 \mathrm{~kg} / \mathrm{m}^{3}$ e tempo máximo $t_{\max }=20 \mathrm{~s}$, discretizado em 2000 passos $(\Delta t=0,01 \mathrm{~s})$.

Figura 97 - Geometria e malha para exemplo de abatimento em material altamente viscoso

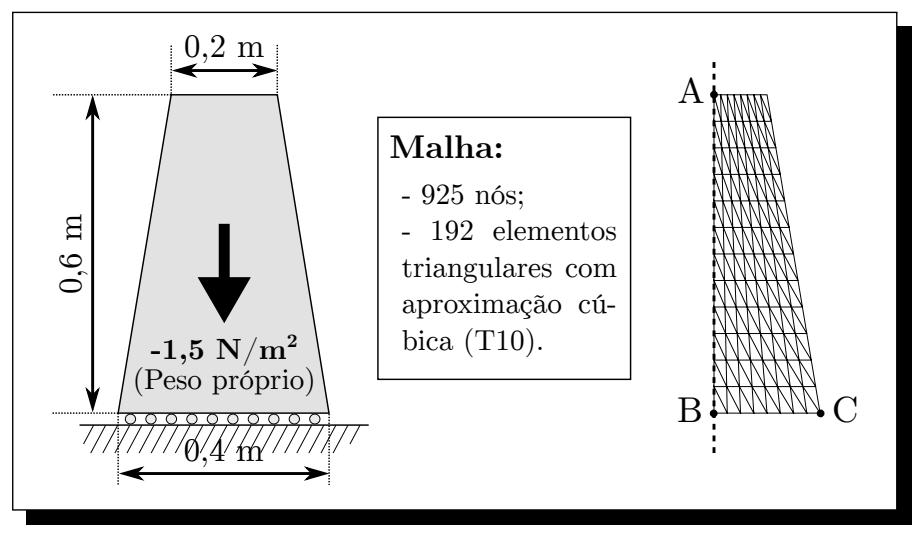

Tabela 16 - Parâmetros de material visco-elasto-plástico para exemplo de abatimento

\begin{tabular}{cccccc}
\hline \multicolumn{5}{c}{ Parâmetros visco-elásticos } \\
\hline$\Lambda^{\infty}(\mathrm{MPa})$ & $\mu^{\infty}(\mathrm{MPa})$ & $\Lambda^{e}(\mathrm{MPa})$ & $\mu^{e}(\mathrm{MPa})$ & $\eta(\mathrm{s} \cdot \mathrm{MPa})$ \\
50 & 0.8 & 200 & 150 & 5 \\
\hline \multicolumn{2}{c}{ Parâmetros de encruamento } & \multicolumn{2}{c}{ Parâmetros de Perzyna/Norton } \\
\hline$\sigma_{Y}(\mathrm{MPa})$ & $c(\mathrm{MPa})$ & $b$ & $\eta_{p}(\mathrm{~s})$ & $\alpha_{p}(\mathrm{MPa})$ & $m$ \\
0.5 & 10 & 1 & 0.5 & 0.5 & 1 \\
\hline
\end{tabular}

Este exemplo é simulado tanto considerando problema quase-estático (dispensando os efeitos inerciais) quanto dinâmico. Os gráficos de deslocamentos são dados na Figura 98 para os dois casos. Nesses, observa-se uma resposta dinâmica suficientemente próxima ao caso quase estático. $O$ mesmo pode ser visto no gráfico da componente $E_{22}$ de deformação de Green-Lagrange, apresentado na Figura 99a. Já o gráfico da componente $\sigma_{22}$ das tensões 
de Cauchy (Figura 99b), apresenta oscilações notáveis, embora ainda seja suficientemente próximo do caso quase estático. Na Figura 100 são apresentadas as configurações deformadas em diversos instantes.

Figura 98 - Deslocamentos para o exemplo de abatimento

(a) Deslocamento horizontal no ponto C

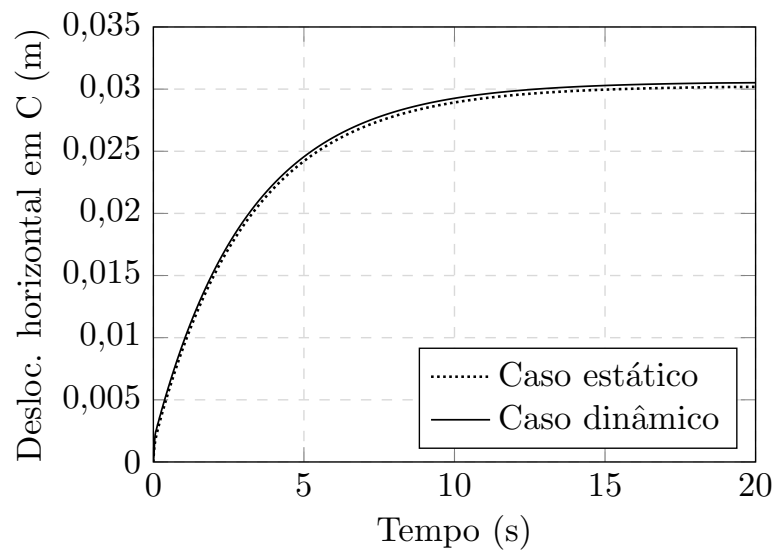

(b) Deslocamento vertical no ponto A

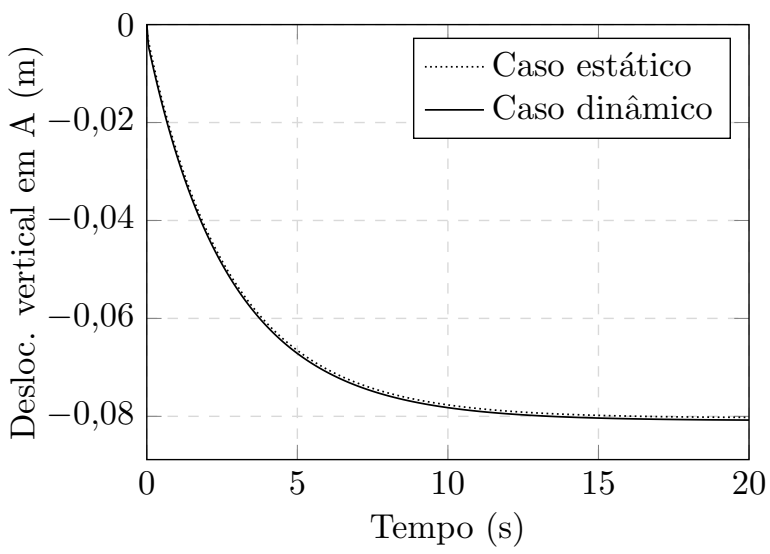

Figura 99 - Deformações e tensões para o exemplo de abatimento

(a) Valores de $E_{22}$ no ponto B

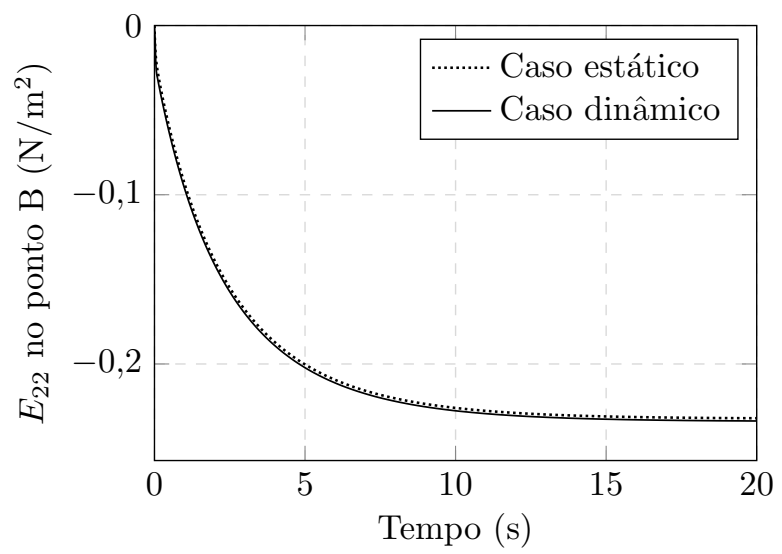

(b) Valores de $\sigma_{22}$ no ponto B

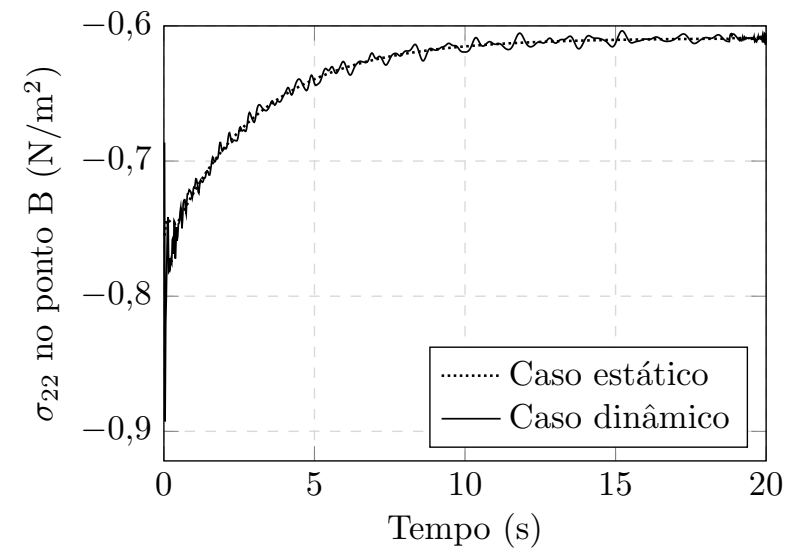

Figura 100 - Configurações deformadas de problema de abatimento, apresentando valores de $\sigma_{22}$ em mapas de cores

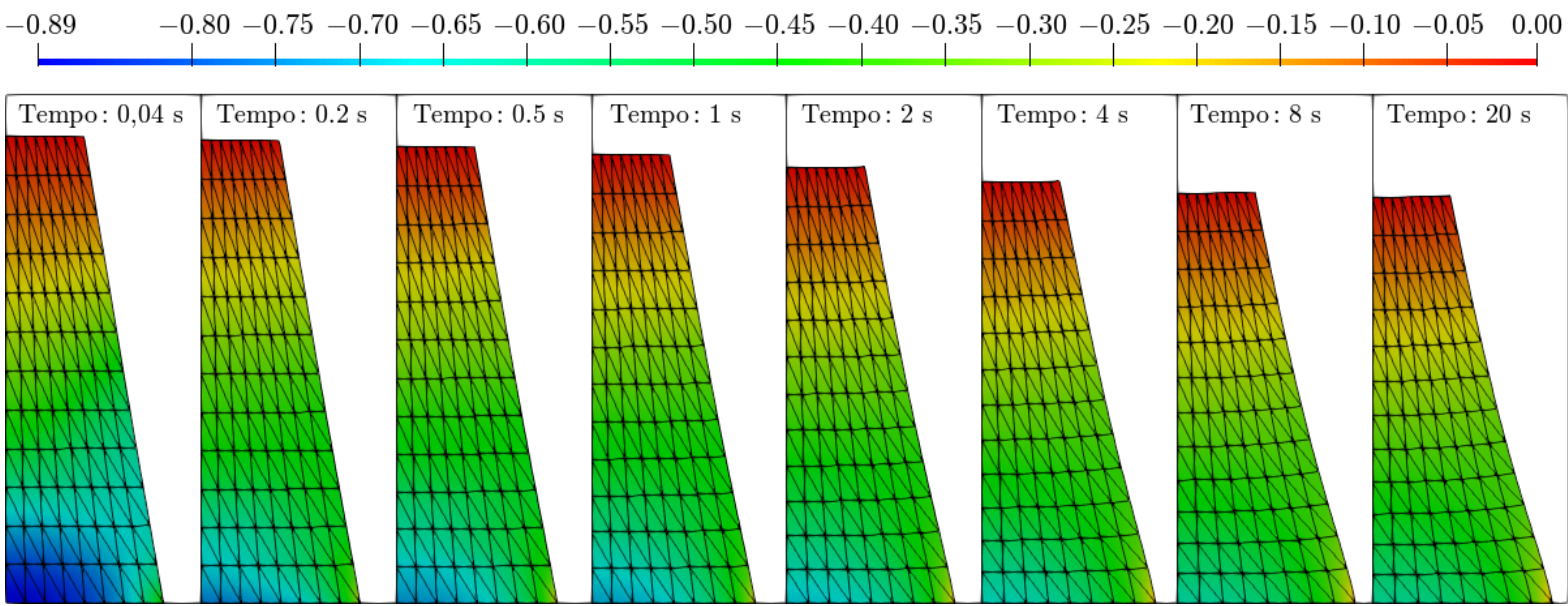




\subsection{Extrusão dinâmica em material altamente viscoso}

Neste exemplo, considera-se um sólido com formato circular e material visco-elastoplástico conforme a Tabela 16, lançado com a força do seu próprio peso contra uma cavidade com $70 \%$ de seu diâmetro, como mostra a Figura 101. Por se tratar de um problema de impacto, consideram-se novamente os parâmetros de Newmark modificados por $\mathrm{Hu}$ (1997), isto é, $\beta=1$ e $\gamma=3 / 2$. O tempo máximo adotado é de $t_{\max }=8,5 \mathrm{~s}$, discretizado em 320000 passos $\left(\Delta t=2,65625 \cdot 10^{-5} \mathrm{~s}\right)$. Considera-se estado plano de tensões e massa $\rho=0,15 \mathrm{~kg} / \mathrm{m}^{3}$.

Figura 101 - Geometria e malha para exemplo de extrusão dinâmica

Geometria:

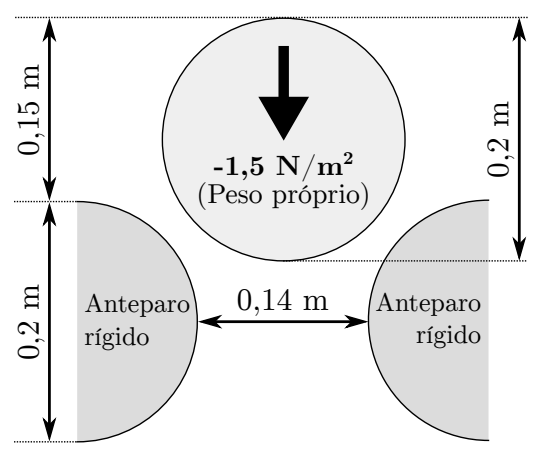

Malha 1:

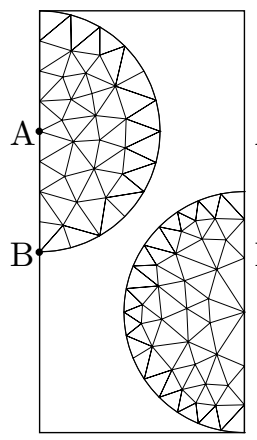

Malha 2:

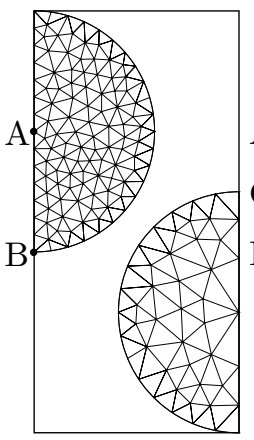

Malha 3:

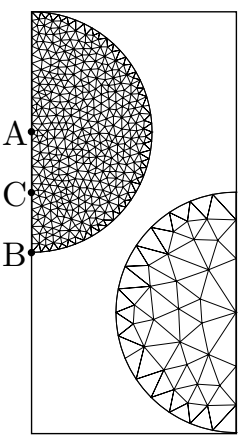

Malha do anteparo: - 358 nós

72 elementos T10 Malha do sólido:

Malha 1:

- 283 nós

- 56 elementos T10

Malha 2:

- 964 nós

202 elementos T10

Malha 3:

- 3625 nós

- 782 elementos T10

Foram utilizadas neste problema três malhas diferentes, conforme mostrado na Figura 101. Os gráficos de deslocamento e deformação plástica nos pontos B e A, respectivamente, são mostrados na Figura 102 para cada uma das malhas. No gráfico de deslocamentos (Figura 102a), as diferenças entre os resultados são praticamente desprezíveis nos instantes iniciais da análise, apresentando um sutil aumento após o período de contato entre o sólido e os anteparos rígidos. No gráfico de deformações plásticas (Figura 102b), é possível notar uma etapa relativamente grande de valores constantes, onde a resposta da Malha 1 difere-se levemente das demais. Após essa etapa, as divergências tornam-se visíveis entre os três casos.

Figura 102 - Deformações e tensões para o exemplo de abatimento

(a) Deslocamento vertical no ponto B

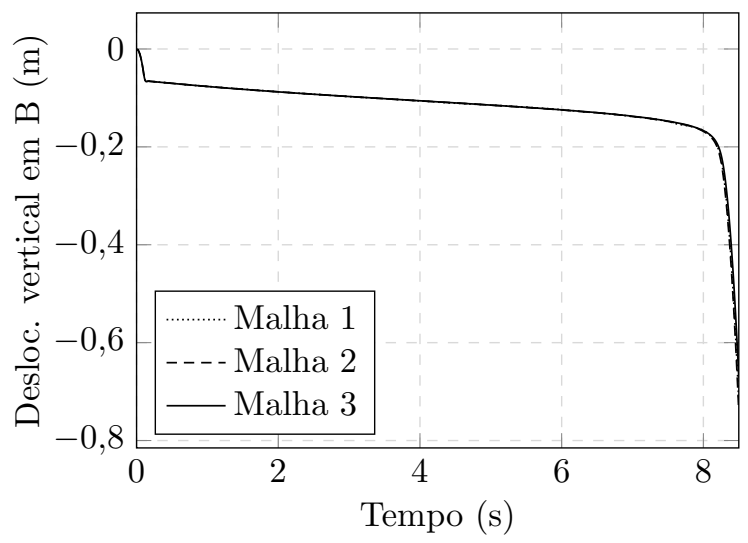

(b) Valores de $E_{22}^{p}$ no ponto A

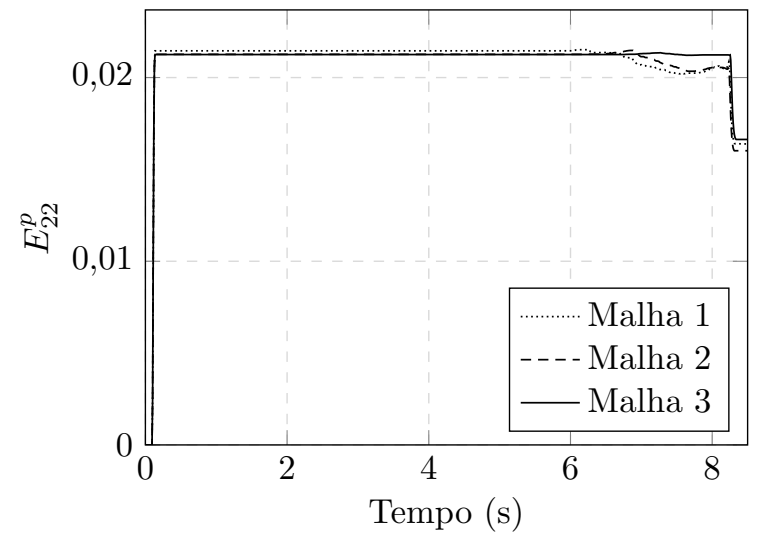


Vale ser dito que as deformações plásticas no ponto A são relativamente pequenas quando comparadas com as deformações totais. Para mostrar isso, apresenta-se na Figura 103a as evoluções das deformações totais, elásticas, plásticas e viscosas neste ponto, para a malha 3. O mesmo é apresentado na Figura 103b para o ponto C (ponto intermediário entre A e B), que pertence a uma região onde as deformações plásticas são mais acentuadas. Os campos de deformação plástica $E_{22}^{p}$ podem ser vistos na Figura 104, onde são mostradas as configurações deformadas do problema em alguns instantes.

Figura 103 - Deformações para o exemplo de extrusão dinâmica com a Malha 3

(a) Deformações em A

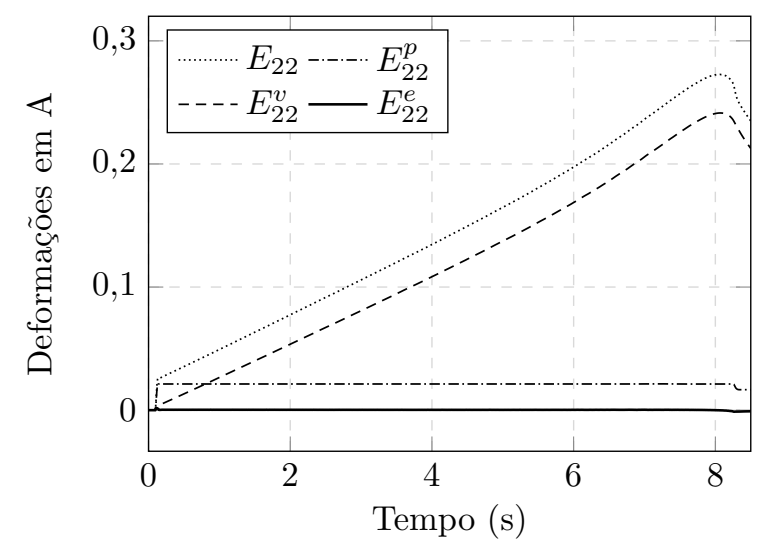

(b) Deformações em C

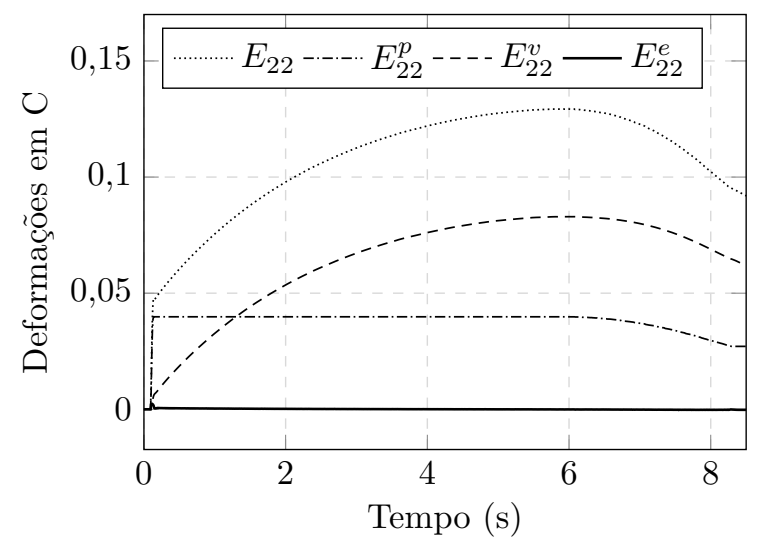

Figura 104 - Configurações deformadas de problema de extrusão dinâmica com a Malha 3, apresentando valores de $E_{22}^{p}$ em mapas de cores

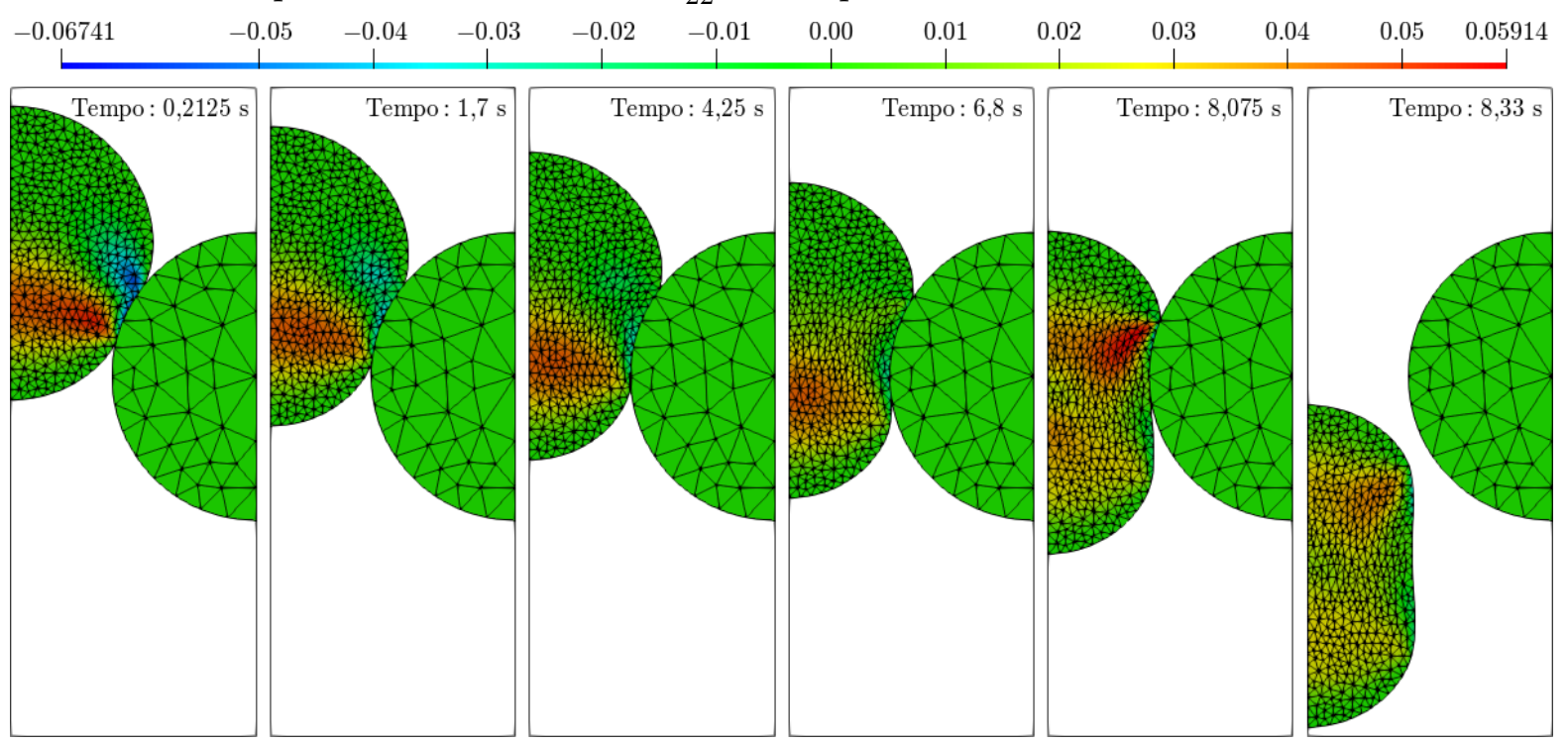




\section{Capítulo 9}

\section{Conclusões}

Neste trabalho, desenvolve-se uma ferramenta numérica para análise bidimensional estática, quase-estática e dinâmica de sólidos com modelos constitutivos visco-elástico, visco-plástico e visco-elasto-plástico, considerando situações de contato. Utiliza-se como base o método dos elementos finitos posicional, conforme descrito em Coda (2018). Para o problema de contato, adota-se estratégia Nó-a-segmento.

\subsection{Modelos constitutivos}

Inicialmente, desenvolve-se um código considerando o modelo constitutivo de SaintVenant Kirchhoff, sendo na sequência incorporados o modelo constitutivo visco-elástico baseado no trabalho de Pascon e Coda (2017), e elasto-plástico baseado nos trabalhos de Svendsen (1998), Dettmer e Reese (2004) e Pascon e Coda (2013a). Ambos se destacam por utilizar uma abordagem Lagrangeana, tomando como base a segunda lei da termodinâmica na forma da inequação de Clausius-Duhem, e partindo da decomposição multiplicativa do gradiente da função mudança de configuração. Uma das diferenças deste trabalho com relação aos previamente citados é a aplicação dos modelos ao caso bidimensional, sendo utilizadas as aproximações do estado plano de deformações e estado plano de tensões.

Nas Seções 4.2.7 e 5.6, verifica-se o código com os exemplos de tensão uniaxial e cisalhamento simples apresentados em Dettmer e Reese (2004) e Pascon e Coda (2017). Deve ser ressaltado que, embora nessas referências tenham sido utilizados elementos tridimensionais e lei constitutiva completa, foi possível obter neste trabalho resultados coincidentes mesmo utilizando elementos bidimensionais, atestando a eficiência das aproximações adotadas. Essa adequação ao caso $2 \mathrm{D}$ é vantajosa por permitir uma redução do custo computacional na modelagem de muitos problemas, fator particularmente importante nesse contexto, tendo em vista o já elevado custo devido à alta complexidade dos modelos descritos.

O modelo elasto-plástico foi, em seguida, generalizado para o caso visco-plástico, onde considera-se a viscosidade associada apenas à parcela plástica de deformações, 
tendo sido utilizado como base o clássico modelo de Perzyna em conjunto com a lei de Norton. Por fim, desenvolve-se um modelo visco-elasto-plástico acoplando os modelos viscoelástico e visco-plástico descritos, utilizando, novamente, a inequação de Clausius-Duhem e a decomposição multiplicativa. Embora leis constitutivas visco-elasto-plásticas sejam largamente aplicadas na literatura, este é o primeiro trabalho a acoplar esses modelos ${ }^{1}$, sendo essa, portanto, a principal contribuição original deste trabalho.

\subsection{Exemplos numéricos}

Além dos exemplos de tensão uniaxial e cisalhamento simples apresentados para verificar o comportamento dos modelos constitutivos, são propostos no Capítulo 8 exemplos numéricos para mostrar as diversas aplicações que o código desenvolvido permite.

No exemplo da seção 8.1 foi investigada a influência das taxas de carregamento para os diversos modelos constitutivos considerados em um caso quase-estático. Por apresentar grandes deformações, esse mesmo exemplo foi utilizado para verificar a influência da discretização temporal sobre a propriedade da conservação do volume inelástico. Para tal, foi realizada uma análise de convergência sobre os valores de $J^{p}$ e $J^{v}$. Os resultados obtidos foram promissores, uma vez que os erros podem ser considerados desprezíveis para aplicações práticas, e, além disso, são reduzidos aproximadamente pela metade à medida que o número de passos é dobrado.

Já no exemplo da seção 8.2 foi investigado o comportamento dissipativo dos diversos modelos constitutivos quando submetidos a uma análise dinâmica. Em cada caso, foram variados os parâmetros de viscosidade $\eta$ e $\eta_{p}$ de forma que pudesse ser obtida uma relação entre esses e a intensidade da dissipação (medida por meio do tempo de estabilização das respostas).

No contexto de conformação de metais, o programa desenvolvido foi aplicado a problemas de dobramento e extrusão. O primeiro caso é apresentado nos exemplos das seções 8.3 e 8.4, onde dois procedimentos diferentes foram realizados para impôr um formato de L em uma chapa metálica. Particularmente, os resultados mostraram que os dois processos resultam em comportamentos totalmente distintos para o Springback. No primeiro exemplo, a configuração final da chapa apresenta um ângulo maior que o desejado entre as extremidades, enquanto no segundo um ângulo menor foi obtido devido à influência do efeito de Bauschinger, mostrando que o modelo de encruamento adotado é capaz de representar o fenômeno adequadamente. O exemplo de extrusão, apresentado na seção 8.5, foi aplicado tanto em materiais do tipo metálico quanto polimérico, onde observou-se no primeiro caso um formato final mais próximo do formato imposto quando comparado ao caso polimérico.

Analisando-se os resultados dos problemas estudados no Capítulo 8 e nas seções 4.3.4 e 6.6, é possível compreender as características dos modelos visco-plástico e visco-

1 Até onde vai o conhecimento do autor. 
elasto-plástico descritos, auxiliando a calibração de seus parâmetros para aplicação em diferentes materiais a partir de dados experimentais. Observa-se que, embora não tenha sido feita uma validação dos modelos (comparação com resultados experimentais), esses apresentam respostas condizentes com o comportamento esperado para esses tipos de materiais.

Para os problemas de impacto, foi observada uma dificuldade de convergência quando utilizado o integrador de Newmark- $\beta$ tradicional, devido às altas frequências presentes nesse tipo de situação, o que já era esperado conforme reportado por outros autores (CHAUDHARY; BATHE, 1986; HU, 1997). Dessa forma, considera-se nos exemplos das seções 8.6 e 8.8 o integrador temporal proposto por Hu (1997), que mostrou-se eficiente para os casos considerados. Tal integrador, entretanto, provoca um amortecimento numérico altamente sensível à discretização temporal adotada, de forma que, para diminuir o erro, deve-se adotar valores de $\Delta t$ muito menores que os normalmente utilizados no integrador de Newmark- $\beta$, conforme constatado pela análise de convergência realizada na seção 8.6.

\subsection{Continuidade da pesquisa e sugestões para trabalhos futuros}

Embora as aproximações de estado plano de deformações e tensões permitam uma resposta mais rápida para problemas que podem ser representados em duas dimensões, são muitos os casos onde tal representação não é possível. Dessa forma, um avanço prioritário na continuidade desta pesquisa é a passagem para o caso tridimensional. Nesse caso, os maiores desafios a serem enfrentados são o custo computacional elevado e o aprimoramento do modelo de contato, que assume particular complexidade no caso 3D.

No que diz respeito ao contato, sugere-se que sejam considerados métodos alternativos, como o Mortar, técnicas baseadas nas trocas de condição de contorno DirichletNeumann ou introdução de elementos de contato. Além disso, é importante a implementação de modelos de atrito e adesão, de forma que os exemplos simulados sejam mais próximos da realidade.

Com relação ao modelo visco-elasto-plástico, sugere-se ainda a validação do mesmo com base em resultados experimentais de modo a se ter uma compreensão real de quais classes de materiais e quais situações são bem representadas por esse modelo, bem como identificar possíveis aspectos que possam ser melhorados.

Ainda que seja possível simular problemas mais complexos envolvendo principalmente processos de manufatura, sugere-se o aperfeiçoamento do modelo constitutivo adotado com a introdução de efeitos térmicos (termo-visco-elasto-plasticidade), considerando ainda a possibilidade de geração de calor devido à dissipação, e transferência por contato. Outro problema que esteve fora do escopo deste trabalho, e que dentre muitas aplicações também ocorre em decorrência de processos de manufatura, é o comportamento anisotrópico dos materiais, cabendo a sugestão da introdução de anisotropia no modelo proposto. 



\section{Referências}

ABDUL-HAMEED, H.; MESSAGER, T.; ZAïRI, F.; NAïT-ABDELAZIZ, M. Large-strain viscoelastic-viscoplastic constitutive modeling of semi-crystalline polymers and model identification by deterministic/evolutionary approach. Computational Materials Science, v. 90, p. 241 - 252, 2014. ISSN 0927-0256.

ARGYRIS, J.; BALMER, H.; DOLTSINIS, J.; DUNNE, P.; HAASE, M.; KLEIBER, M.; MALEJANNAKIS, G.; MLEJNEK, H.-P.; MüLLER, M.; SCHARPF, D. Finite element method - the natural approach. Computer Methods in Applied Mechanics and Engineering, v. 17, n. Part 1, p. 1 - 106, 1979. ISSN 0045-7825.

ARMSTRONG, P. J.; FREDERICK, C. A mathematical representation of the multiaxial Bauschinger effect. Berkeley Nuclear Laboratories, Research \& Development Department Berkeley: Central Electricity Generating Board, 1966. v. 731.

AVANCINI, G. Análise numérica bidimensional de interação fluido-estrutura: Uma formulação posicional baseada em elementos finitos e partículas. 2018. 138 f. Dissertação (Mestrado) - Departamento de Engenharia de Estruturas, Escola de Engenharia de São Carlos, Universidade de São Paulo, São Carlos, 2018.

AYACHIT, U. The ParaView Guide: A Parallel Visualization Application. USA: Kitware, Inc., 2015. ISBN 1930934300, 9781930934306.

BAKER, G. A. Error estimates for finite element methods for second order hyperbolic equations. Siam Journal on Numerical Analysis - SIAM J NUMER ANAL, v. 13, p. 564-576, 091976.

BATHE, K. Finite Element Procedures. [S.l.]: Prentice Hall, 2006. ISBN 9780979004902.

BATHE, K. J.; CHAUDHARY, A. A solution method for planar and axisymmetric contact problems. International Journal for Numerical Methods in Engineering, John Wiley and Sons, Ltd, v. 21, n. 1, p. 65-88, 1985. ISSN 1097-0207.

BATHE, K.-J.; RAMM, E.; WILSON, E. L. Finite element formulations for large deformation dynamic analysis. International Journal for Numerical Methods in Engineering, v. 9, n. 2, p. 353-386, 1975.

BAZILEVS, Y.; TAKIZAWA, K.; TEZDUYAR, T. Computational Fluid-Structure Interaction: Methods and Applications. [S.1.]: Wiley, 2013. (Wiley Series in Computational Mechanics). 
BELGACEM, F.; HILD, P.; LABORDE, P. The mortar finite element method for contact problems. Mathematical and Computer Modelling, v. 28, n. 4-8, p. 263-271, 1998. ISSN 08957177.

BELYTSCHKO, T.; NEAL, M. O. Contact-impact by the pinball algorithm with penalty and lagrangian methods. International Journal for Numerical Methods in Engineering, John Wiley \& Sons, Ltd, v. 31, n. 3, p. 547-572, 1991. ISSN 1097-0207.

BELYTSCHKO, T.; YEH, I. The splitting pinball method for contact-impact problems. Computer Methods in Applied Mechanics and Engineering, v. 105, n. 3, p. 375 393, 1993. ISSN 0045-7825.

BENAARBIA, A.; ROUSE, J.; SUN, W. A thermodynamically-based viscoelasticviscoplastic model for the high temperature cyclic behaviour of 9-12\% cr steels. International Journal of Plasticity, v. 107, p. 100 - 121, 2018. ISSN 0749-6419.

BENSON, D. J.; HALLQUIST, J. O. A single surface contact algorithm for the post-buckling analysis of shell structures. Computer Methods in Applied Mechanics and Engineering, v. 78, n. 2, p. $141-163$, 1990. ISSN 0045-7825.

BERNARDI, C.; DEBIT, N.; MADAY, Y. Coupling finite element and spectral methods: First results. Mathematics of Computation, American Mathematical Society, v. 54, n. 189, p. 21-39, 1990. ISSN 00255718, 10886842.

BERNARDI, C.; MADAY, Y.; PATERA, A. T. A new nonconforming approach to domain decomposition: The mortar element method. In: Nonlinear partial differential equations and their applications. Collège de France Seminar, volume XI. Lectures presented at the weekly seminar on applied mathematics. [S.l.: s.n.], 1994. p. 13-51. ISBN 0-582-23800-5.

BONET, J.; WOOD, R. Nonlinear Continuum Mechanics for Finite Element Analysis. [S.l.]: Cambridge University Press, 1997. ISBN 9780521572729.

BONET, J.; WOOD, R.; MAHANEY, J.; HEYWOOD, P. Finite element analysis of air supported membrane structures. Computer Methods in Applied Mechanics and Engineering, v. 190, n. 5, p. 579 - 595, 2000. ISSN 0045-7825.

BREPOLS, T.; VLADIMIROV, I. N.; REESE, S. Numerical comparison of isotropic hypo- and hyperelastic-based plasticity models with application to industrial forming processes. International Journal of Plasticity, v. 63, p. 18 - 48, 2014. ISSN 0749-6419. Deformation Tensors in Material Modeling in Honor of Prof. Otto T. Bruhns.

CAREGliO, C.; CAnAles, C.; GARINO, C. G.; Mirasso, A.; PONTHOT, J. P. A numerical study of hypoelastic and hyperelastic large strain viscoplastic perzyna type models. Acta Mechanica, v. 227, n. 11, p. 3177-3190, Nov 2016. ISSN 1619-6937.

CARPENTER, N. J.; TAYLOR, R. L.; KATONA, M. G. Lagrange constraints for transient finite element surface contact. International Journal for Numerical Methods in Engineering, John Wiley \& Sons, Ltd, v. 32, n. 1, p. 103-128, 1991. ISSN 1097-0207.

CHABOCHE, J. Time-independent constitutive theories for cyclic plasticity.

International Journal of Plasticity, v. 2, n. 2, p. 149 - 188, 1986. 
CHABOCHE, J. A review of some plasticity and viscoplasticity constitutive theories. International Journal of Plasticity, v. 24, n. 10, p. 1642 - 1693, 2008. ISSN 0749-6419. Special Issue in Honor of Jean-Louis Chaboche.

CHAKRABARTY, J. Theory of plasticity. [S.l.]: McGraw-Hill Ryerson, Limited, 1987. (Engineering mechanics series). ISBN 9780070103924.

CHAUDHARY, A. B.; BATHE, K.-J. A solution method for static and dynamic analysis of three-dimensional contact problems with friction. Computers and Structures, v. 24, n. 6 , p. $855-873,1986$. ISSN 0045-7949.

CHRISTENSEN, R. Theory of Viscoelasticity: Second Edition. [S.l.]: Dover Publications, 2013. (Dover Civil and Mechanical Engineering). ISBN 9780486318967.

CLOUGH, R. The finite element method in plane stress analysis. In: SECOND ASCE CONFERENCE ON ELECTRONIC COMPUTATION. Pittsburgh, PA, 1960. p. 345-378.

CODA, H. B. Análise não linear geométrica de sólidos e estruturas: Uma formulação posicional baseada no MEF. 2003. Tese (Tese para concurso de professor titular) - Departamento de Engenharia de Estruturas, Escola de Engenharia de São Carlos, Universidade de São Paulo, São Carlos, 2003.

CODA, H. B. O Método dos elementos finitos posicional: sólidos e estruturas não linearidade geométrica e dinâmica. São Carlos: EESC/USP, 2018. 284 p. ISBN 978-85-8023-068-0.

CODA, H. B.; GRECO, M. A simple FEM formulation for large deflection 2D frame analysis based on position description. Computer Methods in Applied Mechanics and Engineering, v. 193, n. 33-35, p. 3541-3557, 2004. ISSN 00457825.

CODA, H. B.; PACCOLA, R. R. An alternative positional FEM formulation for geometrically non-linear analysis of shells: Curved triangular isoparametric elements. Computational Mechanics, v. 40, n. 1, p. 185-200, 2007. ISSN 01787675.

CODA, H. B.; PACCOLA, R. R. Unconstrained finite element for geometrical nonlinear dynamics of shells. Mathematical Problems in Engineering, 2009. ISSN 1024123X.

CODA, H. B.; PACCOLA, R. R. A total-Lagrangian position-based FEM applied to physical and geometrical nonlinear dynamics of plane frames including semi-rigid connections and progressive collapse. Finite Elements in Analysis and Design, v. 91, p. 1-15, 2014. ISSN 0168874X.

CODA, H. B.; SAMPAIO, M. S.; PACCOLA, R. R. A FEM continuous transverse stress distribution for the analysis of geometrically nonlinear elastoplastic laminated plates and shells. Finite Elements in Analysis and Design, v. 101, p. 15-33, 2015. ISSN 0168874X.

COLEMAN, B. D.; NOLL, W. The thermodynamics of elastic materials with heat conduction and viscosity. Archive for Rational Mechanics and Analysis, v. 13, n. 1, p. 167-178, Dec 1963. ISSN 1432-0673.

CRISFIELD, M. A. Non-Linear Finite Element Analysis of Solids and Structures: Advanced Topics. 1st. ed. New York, NY, USA: John Wiley and Sons, Inc., 1997. ISBN 047195649X. 
DAGUM, L.; MENON, R. OpenMP: An industry-standard API for shared-memory programming. IEEE Comput. Sci. Eng., IEEE Computer Society Press, Los Alamitos, CA, USA, v. 5, n. 1, p. 46-55, jan. 1998. ISSN 1070-9924.

DARABI, M. K.; AL-RUB, R. K. A.; MASAD, E. A.; HUANG, C.-W.; LITTLE, D. N. A thermo-viscoelastic--viscoplastic-viscodamage constitutive model for asphaltic materials. International Journal of Solids and Structures, v. 48, n. 1, p. $191-207,2011$. ISSN 0020-7683.

DETTMER, W.; REESE, S. On the theoretical and numerical modelling of armstrongfrederick kinematic hardening in the finite strain regime. Computer Methods in Applied Mechanics and Engineering, v. 193, n. 1, p. 87 - 116, 2004.

DOGUI, A.; SIDOROFF, F. Kinematic hardening in large elastoplastic strain. Engineering Fracture Mechanics, v. 21, n. 4, p. 685 - 695, 1985.

DRESCHER, A.; KRINGOS, N.; SCARPAS, T. On the behavior of a parallel elasto-visco-plastic model for asphaltic materials. Mechanics of Materials, v. 42, n. 2, p. 109 - 117, 2010. ISSN 0167-6636.

DUNAVANT, D. A. High degree efficient symmetrical Gaussian quadrature rules for the triangle. International Journal for Numerical Methods in Engineering, v. 21, p. 1129-1148, 1985.

DUVAUT, G.; LIONS, J. Inequalities in mechanics and physics. [S.l.]:

Springer-Verlag, 1976. (Grundlehren der mathematischen Wissenschaften). ISBN 9783540073277.

ETEROVIC, A. L.; BATHE, K. J. A note on the use of the additive decomposition of the strain tensor in finite deformation inelasticity. Computer Methods in Applied Mechanics and Engineering, v. 93, n. 1, p. 31 - 38, 1991. ISSN 0045-7825.

FERNANDES, J. W. D.; CODA, H. B.; SANCHES, R. A. K. Ale incompressible fluid-shell coupling based on a higher-order auxiliary mesh and positional shell finite element. Computational Mechanics, Aug 2018. ISSN 1432-0924.

FINDLEY, W.; LAI, J.; ONARAN, K. Creep and Relaxation of Nonlinear Viscoelastic Materials: With an Introduction to Linear Viscoelasticity. [S.1.]: Dover, 1989. (Dover Civil and Mechanical Engineering). ISBN 9780486660165.

FISCHER, K. A.; WRIGGERS, P. Frictionless 2D contact formulations for finite deformations based on the mortar method. Computational Mechanics, v. 36, n. 3, p. 226-244, Aug 2005. ISSN 1432-0924.

FISCHER, K. A.; WRIGGERS, P. Mortar based frictional contact formulation for higher order interpolations using the moving friction cone. Computer Methods in Applied Mechanics and Engineering, v. 195, n. 37-40, p. 5020-5036, 2006. ISSN 00457825.

GARINO, C. G.; VAIRO, M. R.; FAGéS, S. A.; MIRASSO, A.; PONTHOT, J.-P. Numerical simulation of finite strain viscoplastic problems. Journal of Computational and Applied Mathematics, v. 246, p. 174 - 184, 2013. ISSN 0377-0427. Fifth International Conference on Advanced COmputational Methods in ENgineering (ACOMEN 2011). 
GEUZAINE, C.; REMACLE, J.-F. Gmsh: a three-dimensional finite element mesh generator with built-in pre- and post-processing facilities. International Journal for Numerical Methods in Engineering, v. 79, p. 1309 - 1331, 2009.

GIBSON, I.; ROSEN, D.; STUCKER, B. Additive Manufacturing Technologies: 3D Printing, Rapid Prototyping, and Direct Digital Manufacturing. [S.l.]: Springer New York, 2014. ISBN 9781493921133.

GRECO, M. Análise de problemas de contato/impacto em estruturas de comportamento não linear pelo método dos elementos finitos. 2004. 153 f. Tese (Doutorado) - Departamento de Engenharia de Estruturas, Escola de Engenharia de São Carlos, Universidade de São Paulo, São Carlos, 2004.

GRECO, M.; CODA, H. B. Positional FEM formulation for flexible multi-body dynamic analysis. Journal of Sound and Vibration, v. 290, n. 3-5, p. 1141-1174, 2006. ISSN 10958568.

GRECO, M.; GESUALDO, F.; VENTURINI, W.; CODA, H. Nonlinear positional formulation for space truss analysis. Finite Elements in Analysis and Design, v. 42, n. 12 , p. 1079 - 1086, 2006. ISSN 0168-874X.

GREEN, M. S.; TOBOLSKY, A. V. A new approach to the theory of relaxing polymeric media. The Journal of Chemical Physics, v. 14, n. 2, p. 80-92, 1946.

GUDIMETLA, M. R.; DOGHRI, I. A finite strain thermodynamically-based constitutive framework coupling viscoelasticity and viscoplasticity with application to glassy polymers. International Journal of Plasticity, v. 98, p. 197 - 216, 2017. ISSN 0749-6419.

HALLQUIST, J.; GOUDREAU, G.; BENSON, D. Sliding interfaces with contact-impact in large-scale lagrangian computations. Computer Methods in Applied Mechanics and Engineering, v. 51, n. 1, p. $107-137,1985$. ISSN 0045-7825.

HALLQUIST, J. O. NIKE2D - an implicit, finite-deformation finite-element code for analyzing the static and dynamic response of two-dimensional solids. Berkeley, CA: Lawrence Berkeley Nat. Lab., 1979.

HARTMANN, S.; RAMM, E. A mortar based contact formulation for non-linear dynamics using dual Lagrange multipliers. Finite Elements in Analysis and Design, v. 44, n. 5, p. 245-258, 2008. ISSN 0168874X.

HASANPOUR, K.; ZIAEI-RAD, S.; MAHZOON, M. A large deformation framework for compressible viscoelastic materials: Constitutive equations and finite element implementation. International Journal of Plasticity, v. 25, n. 6, p. $1154-1176,2009$. ISSN 0749-6419.

HAUPT, P. On the concept of an intermediate configuration and its application to a representation of viscoelastic-plastic material behavior. International Journal of Plasticity, v. 1, n. 4, p. 303 - 316, 1985.

HEERES, O. M.; SUIKER, A. S.; BORST, R. de. A comparison between the perzyna viscoplastic model and the consistency viscoplastic model. European Journal of Mechanics - A/Solids, v. 21, n. 1, p. 1 - 12, 2002. ISSN 0997-7538. 
HILL, R. The Mathematical Theory of Plasticity. [S.l.]: Clarendon Press, 1998. (Oxford classic texts in the physical sciences). ISBN 9780198503675.

HOLMES, D. W.; LOUGHRAN, J. G.; SUEHRCKE, H. Constitutive model for large strain deformation of semicrystalline polymers. Mechanics of Time-Dependent Materials, v. 10, n. 4, p. 281-313, Dec 2006. ISSN 1573-2738.

HOLZAPFEL, G. Nonlinear Solid Mechanics: A Continuum Approach for Engineering. [S.1.]: Wiley, 2000.

HOLZAPFEL, G. a. On large strain viscoelasticity: continuum formulation and finite element applications to elastomeric structures. International Journal for Numerical Methods in Engineering, v. 39, n. 22, p. 3903-3926, 1996. ISSN 0029-5981.

HOLZAPFEL, G. A.; SIMO, J. C. A new viscoelastic constitutive model for continuous media at finite thermomechanical changes. International Journal of Solids and Structures, v. 33, n. 20, p. 3019 - 3034, 1996. ISSN 0020-7683.

HSL. A collection of fortran codes for large scale scientific computation. 2013. Disponível em: <http://www.hsl.rl.ac.uk/>.

HU, N. A solution method for dynamic contact problems. v. 63, p. 1053-1063, 061997.

HUBER, N.; TSAKMAKIS, C. Finite deformation viscoelasticity laws. Mechanics of Materials, v. 32, p. 1-18, 012000.

HUGHES, T.; TAYLOR, R.; SACKMAN, J.; CURNIER, A.; KANOKNUKULCHAI, W. A finite element method for a class of contact-impact problems. Computer Methods in Applied Mechanics and Engineering, Elsevier, v. 8, n. 3, p. 249-276, 1976. ISSN 0045-7825.

IBRAHIMBEGOVIć, A.; CHORFI, L. Viscoplastic model at finite deformations with combined isotropic and kinematic hardening. Computers \& Structures - COMPUT STRUCT, v. 77, p. 509-525, 072000.

JOHNSON, K. Contact Mechanics. [S.l.]: Cambridge University Press, 1987.

JOHNSON, W.; MELLOR, P. Engineering Plasticity. [S.l.]: Ellis Horwood Limited, 1983. (Ellis Horwood series in engineering science). ISBN 9780853123460.

KHAN, a. S.; HUANG, S. Continuum Theory of Plasticity. New York WileyInterscience 1995, p. 440, 1995. ISSN 1520-5207.

KIM, J. S.; MULIANA, A. H. A time-integration method for the viscoelastic-viscoplastic analyses of polymers and finite element implementation. International Journal for Numerical Methods in Engineering, v. 79, n. 5, p. 550-575, 2009.

KIRCHNER, E.; REESE, S.; WRIGGERS, P. A finite element method for plane stress problems with large elastic and plastic deformations. Communications in Numerical Methods in Engineering, v. 13, n. 12, p. 963-976, 1997.

KRÖNER, E. Allgemeine kontinuumstheorie der versetzungen und eigenspannungen.

Archive for Rational Mechanics and Analysis, v. 4, n. 4, p. 273-334, 1960. 
KROON, M. A constitutive framework for modelling thin incompressible viscoelastic materials under plane stress in the finite strain regime. Mechanics of Time-Dependent Materials, v. 15, n. 4, p. 389-406, Nov 2011. ISSN 1573-2738.

LAI, J.; BAKKER, A. An integral constitutive equation for nonlinear plasto-viscoelastic behavior of high-density polyethylene. Polymer Engineering \& Science, v. 35, n. 17, p. $1339-1347,1995$.

LAMMENS, N.; KERSEMANS, M.; BAERE, I. D.; PAEPEGEM, W. V. On the visco-elasto-plastic response of additively manufactured polyamide-12 (pa-12) through selective laser sintering. Polymer Testing, v. 57, p. 149 - 155, 2017. ISSN 0142-9418.

LEE, E. H. Elastic-plastic deformation at finite strains. Journal of Applied Mechanics, v. 36, n. 1, p. 1-6, 1969.

LEMAITRE, J.; CHABOCHE, J. Mechanics of Solid Materials. [S.l.]: Cambridge University Press, 1985.

LEMAITRE, J. J. Handbook of materials behavior models. [S.l.]: Academic Press, 2001. ISBN 9780124433410.

LION, A. A physically based method to represent the thermo-mechanical behaviour of elastomers. Acta Mechanica, v. 123, n. 1, p. 1-25, Mar 1997. ISSN 1619-6937.

LION, A. Constitutive modelling in finite thermoviscoplasticity: a physical approach based on nonlinear rheological models. International Journal of Plasticity, v. 16, n. 5, p. $469-494,2000$. ISSN 0749-6419.

LUBLINER, J. A model of rubber viscoelasticity. Mechanics Research Communications, v. 12, n. 2, p. 93 - 99, 1985. ISSN 0093-6413.

LÜHRS, G.; HARTMANN, S.; HAUPT, P. On the numerical treatment of finite deformations in elastoviscoplasticity. Computer Methods in Applied Mechanics and Engineering, v. 144, n. 1, p. 1-21, 1997.

MACIEL, D. N. Análise de problemas elásticos não-lineares geométricos empregando o método dos elementos finitos posicional. 2008. 162 f. Tese (Doutorado) - Departamento de Engenharia de Estruturas, Escola de Engenharia de São Carlos, Universidade de São Paulo, São Carlos, 2008.

MADEIRA, R. H.; CODA, H. B. Kelvin viscoelasticity and lagrange multipliers applied to the simulation of nonlinear structural vibration control. Latin American Journal of Solids and Structures, v. 13, n. 5, p. 964-991, 2016. ISSN 16797825.

MANDEL, J. Thermodynamics and plasticity. In: DOMINGOS, J. J. D.; NINA, M. N. R.; WHITELAW, J. H. (Ed.). Foundations of Continuum Thermodynamics. London: Macmillan Education UK, 1973. p. 283-304. ISBN 978-1-349-02235-9.

MARQUES, G. C. S. C. Estudo e desenvolvimento de código computacional baseado no MEF para análise dinâmica não linear geométrica de sólidos bidimensionais. 2006. 93 f. Dissertação (Mestrado) — Departamento de Engenharia de Estruturas, Escola de Engenharia de São Carlos, Universidade de São Paulo, São Carlos, 2006. 
MESQUITA, A. D.; CODA, H. B. Alternative Kelvin viscoelastic procedure for finite elements. Applied Mathematical Modelling, v. 26, n. 4, p. 501-516, 2002. ISSN $0307904 X$.

MESQUITA, A. D.; CODA, H. B. New methodology for the treatment of two dimensional viscoelastic coupling problems. Computer Methods in Applied Mechanics and Engineering, v. 192, n. 16-18, p. 1911-1927, 2003. ISSN 00457825.

MESQUITA, A. D.; CODA, H. B. A boundary element methodology for viscoelastic analysis: Part II without cells. Applied Mathematical Modelling, v. 31, n. 6, p. 1171-1185, 2007. ISSN 0307904X.

MäHLER, L.; EKH, M.; RUNESSON, K. A class of thermo-hyperelastic-viscoplastic models for porous materials: theory and numerics. International Journal of Plasticity, v. 17, n. 7, p. 943 - 969, 2001. ISSN 0749-6419.

MILED, B.; DOGHRI, I.; DELANNAY, L. Coupled viscoelastic-viscoplastic modeling of homogeneous and isotropic polymers: Numerical algorithm and analytical solutions. Computer Methods in Applied Mechanics and Engineering, v. 200, n. 47, p. 3381 - 3394, 2011. ISSN 0045-7825.

MINSKI, R. L. Aprimoramento de formulação de identificação e solução do impacto bidimensional entre estrutura e anteparo rígido. 2008. 126 f. Dissertação (Mestrado) - Departamento de Engenharia de Estruturas, Escola de Engenharia de São Carlos, Universidade de São Paulo, São Carlos, 2008.

MOLLICA, F.; RAJAGOPAL, K.; SRINIVASA, A. The inelastic behavior of metals subject to loading reversal. International Journal of Plasticity, v. 17, n. 8, p. 1119 $1146,2001$.

NEWMARK, N. A Method of Computation for Structural Dynamics. [S.l.]: American Society of Civil Engineers, 1959. (A Method of Computation for Structural Dynamics, № $179-181)$.

NGUYEN, V. D.; LANI, F.; PARDOEN, T.; MORELlE, X.; NOELS, L. A large strain hyperelastic viscoelastic-viscoplastic-damage constitutive model based on a multi-mechanism non-local damage continuum for amorphous glassy polymers. International Journal of Solids and Structures, v. 96, 062016.

OGDEN, R. Non-linear Elastic Deformations. [S.l.]: Dover Publications, 1997. (Dover Civil and Mechanical Engineering). ISBN 9780486696485.

OLIVEIRA, M.; ALVES, J.; CHAPARRO, B.; MENEZES, L. Study on the influence of work-hardening modeling in springback prediction. International Journal of Plasticity, v. 23, n. 3, p. 516 - 543, 2007.

OTtOSEN, N.; RISTINMAA, M. The Mechanics of Constitutive Modeling. [S.l.]: Elsevier Science, 2005. (The Mechanics of Constitutive Modeling). ISBN 9780080525693.

PAPADOPOULOS, P.; TAYLOR, R. L. A mixed formulation for the finite element solution of contact problems. Computer Methods in Applied Mechanics and Engineering, v. 94, n. 3, p. 373 - 389, 1992. ISSN 0045-7825. 
PASCON, J. High-order triangular finite elements applied to visco-hyperelastic materials under plane stress. Journal of the Brazilian Society of Mechanical Sciences and Engineering, v. 40, p. 40:535, 112018.

PASCON, J.; CODA, H. Large deformation analysis of elastoplastic homogeneous materials via high order tetrahedral finite elements. Finite Elements in Analysis and Design, v. 76, p. $21-38,2013 a$.

PASCON, J. P. Modelos constitutivos para materiais hiperelásticos: estudo e implementação computacional. 2008. 230 f. Dissertação (Mestrado) — Departamento de Engenharia de Estruturas, Escola de Engenharia de São Carlos, Universidade de São Paulo, São Carlos, 2008.

PASCON, J. P. Sobre modelos constitutivos não lineares para materiais com gradação funcional exibindo grandes deformações: implementação numérica em formulação não linear geométrica. 2012. 480 f. Tese (Doutorado) — Departamento de Engenharia de Estruturas, Escola de Engenharia de São Carlos, Universidade de São Paulo, São Carlos, 2012.

PASCON, J. P.; CODA, H. B. High-order tetrahedral finite elements applied to large deformation analysis of functionally graded rubber-like materials. Applied Mathematical Modelling, v. 37, n. 20-21, p. 8757-8775, 2013b. ISSN 0307904X.

PASCON, J. P.; CODA, H. B. Large deformation analysis of functionally graded elastoplastic materials via solid tetrahedral finite elements. Computers \& Structures, v. 146, p. 59-75, 2015. ISSN 00457949.

PASCON, J. P.; CODA, H. B. Finite deformation analysis of visco-hyperelastic materials via solid tetrahedral finite elements. Finite Elements in Analysis and Design, v. 133, p. $25-41,2017$. ISSN 0168-874X.

PERZYNA, P. Fundamental problems in viscoplasticity. In: CHERNYI, G.; DRYDEN, H.; GERMAIN, P.; HOWARTH, L.; OLSZAK, W.; PRAGER, W.; PROBSTEIN, R.; ZIEGLER, H. (Ed.). [S.l.]: Elsevier, 1966, (Advances in Applied Mechanics, v. 9). p. 243 377.

PETITEAU, J.-C.; VERRON, E.; OTHMAN, R.; SOURNE, H. L.; SIGRIST, J.-F.; BARRAS, G. Large strain rate-dependent response of elastomers at different strain rates: convolution integral vs. internal variable formulations. Mechanics of Time-Dependent Materials, v. 17, n. 3, p. 349-367, Aug 2013. ISSN 1573-2738.

PIEDADE NETO, D. Sobre estratégias de resolução numérica de problemas de contato. 2009. 149 f. Dissertação (Mestrado) - Departamento de Engenharia de Estruturas, Escola de Engenharia de São Carlos, Universidade de São Paulo, São Carlos, 2009 .

PIEDADE NETO, D. Sobre o Método dos Elementos Finitos Generalizados em análises da Mecânica dos Sólidos não-linear. 2013. 212 f. Tese (Doutorado) - Departamento de Engenharia de Estruturas, Escola de Engenharia de São Carlos, Universidade de São Paulo, São Carlos, 2013. 
POLIZZOTTO, C. A maximum reduced dissipation principle for nonassociative plasticity. Atti della Accademia Nazionale dei Lincei. Classe di Scienze Fisiche, Matematiche e Naturali. Rendiconti Lincei. Matematica e Applicazioni, Accademia Nazionale dei Lincei, v. 9, n. 2, p. 115-129, 61998.

PONTHOT, J. Unified stress update algorithms for the numerical simulation of large deformation elasto-plastic and elasto-viscoplastic processes. International Journal of Plasticity, v. 18, n. 1, p. 91 - 126, 2002. ISSN 0749-6419.

POPP, A.; GEE, M. W.; WALL, W. A. A finite deformation mortar contact formulation using a primal-dual active set strategy. International Journal for Numerical Methods in Engineering, v. 79, n. 11, p. 1354-1391, 2009. ISSN 00295981.

PRAUD, F.; CHATZIGEORGIOU, G.; BIKARD, J.; MERAGHNI, F. Phenomenological multi-mechanisms constitutive modelling for thermoplastic polymers, implicit implementation and experimental validation. Mechanics of Materials, v. 114, p. 9 - 29, 2017. ISSN 0167-6636.

PUSO, M. A.; LAURSEN, T. A. A mortar segment-to-segment contact method for large deformation solid mechanics. Computer Methods in Applied Mechanics and Engineering, v. 193, n. 6, p. $601-629,2004$. ISSN 0045-7825.

REESE, S.; GOVINDJEE, S. Theoretical and numerical aspects in the thermo-viscoelastic material behaviour of rubber-like polymers. Mechanics of Time-Dependent Materials, v. 1, n. 4, p. 357-396, Dec 1997. ISSN 1573-2738.

RIGOBELLO, R. Desenvolvimento e aplicação de código computacional para análise de estruturas de aço aporticadas em situação de incêndio. 2011. 272 f. Tese (Doutorado) - Departamento de Engenharia de Estruturas, Escola de Engenharia de São Carlos, Universidade de São Paulo, São Carlos, 2011.

RUBERT, J. B. Grandes deformações e anisotropia por tensores de mapeamento aplicados a problemas de materiais compostos e na conformação de metais. 1997. 237 f. Tese (Doutorado) — Departamento de Engenharia de Estruturas, Escola de Engenharia de São Carlos, Universidade de São Paulo, São Carlos, 1997.

SANCHES, R. A.; CODA, H. B. Unconstrained vector nonlinear dynamic shell formulation applied to fluid structure interaction. Computer Methods in Applied Mechanics and Engineering, v. 259, p. 177 - 196, 2013. ISSN 0045-7825.

SANCHES, R. A. A. K.; CODA, H. B. Flexible Multibody Dynamics Finite Element Formulation Applied to Structural Progressive Collapse Analysis. Latin American Journal of Solids and Structures, scielo, v. 14, p. 52 - 71, 01 2017. ISSN 1679-7825.

SANCHES, R. A. K.; CODA, H. B. On fluid-shell coupling using an arbitrary lagrangian-eulerian fluid solver coupled to a positional lagrangian shell solver. Applied Mathematical Modelling, v. 38, n. 14, p. 3401 - 3418, 2014. ISSN 0307-904X.

SHAHSAVARI, H.; NAGHDABADI, R.; BAGHANI, M.; SOHRABPOUR, S. A finite deformation viscoelastic-viscoplastic constitutive model for self-healing materials. Smart Materials and Structures, IOP Publishing, v. 25, n. 12, p. 125027, nov 2016. 
SIDOROFF, F. Nonlinear viscoelastic model with an intermediate configuration. [un modele viscoelastique non lineaire avec configuration intermediaire.]. J Mec, v. 13, n. 4, p. 679-713, 1974. Cited By 103.

SIMO, J. Algorithms for static and dynamic multiplicative plasticity that preserve the classical return mapping schemes of the infinitesimal theory. Computer Methods in Applied Mechanics and Engineering, v. 99, n. 1, p. $61-112$, 1992. ISSN 0045-7825.

SIMO, J.; HUGHES, T. Computational Inelasticity. [S.l.]: Springer New York, 2000. (Interdisciplinary Applied Mathematics). ISBN 9780387975207.

SIMO, J. C. On a fully three-dimensional finite-strain viscoelastic damage model: Formulation and computational aspects. Computer Methods in Applied Mechanics and Engineering, v. 60, n. 2, p. 153-173, 1987. ISSN 00457825.

SIMO, J. C.; WRIGGERS, P.; TAYLOR, R. L. A perturbed lagrangian formulation for the finite element solution of contact problems. Computer Methods in Applied Mechanics and Engineering, v. 50, n. 2, p. 163 - 180, 1985. ISSN 0045-7825.

SOLBERG, J. M.; PAPADOPOULOS, P. A finite element method for contact/impact. Finite Elements in Analysis and Design, v. 30, n. 4, p. $297-311,1998$. ISSN 0168-874X.

SVENDSEN, B. A thermodynamic formulation of finite-deformation elastoplasticity with hardening based on the concept of material isomorphism. International Journal of Plasticity, v. 14, n. 6, p. $473-488,1998$.

SVENDSEN, B.; ARNDT, S.; KLINGBEIL, D.; SIEVERT, R. Hyperelastic models for elastoplasticity with non-linear isotropic and kinematic hardening at large deformation. International Journal of Solids and Structures, v. 35, n. 25, p. 3363 - 3389, 1998.

TALLEC, P. L.; RAHIER, C.; KAISS, A. Three-dimensional incompressible viscoelasticity in large strains: Formulation and numerical approximation. Computer Methods in Applied Mechanics and Engineering, v. 109, n. 3, p. 233 - 258, 1993. ISSN 0045-7825.

TAYLOR, R. L.; PAPADOPOULOS, P. On a finite element method for dynamic contact/impact problems. International Journal for Numerical Methods in Engineering, John Wiley \& Sons, Ltd, v. 36, n. 12, p. 2123-2140, 1993. ISSN 1097-0207.

TSAKMAKIS, C.; WILLUWEIT, A. Use of the elastic predictor-plastic corrector method for integrating finite deformation plasticity laws. In: HUTTER, K.; BAASER, H. (Ed.). Deformation and Failure in Metallic Materials. Berlin, Heidelberg: Springer Berlin Heidelberg, 2003. p. 79-106.

TUR, M.; GINER, E.; FUENMAYOR, F.; WRIGGERS, P. 2D contact smooth formulation based on the mortar method. Computer Methods in Applied Mechanics and Engineering, v. 247-248, p. 1 - 14, 2012.

TURNER, M.; CLOUGH, R.; MARTIN, H.; TOPP, L. Stiffness and deflection analysis of complex structures. Journal of Aeronautical Sciences, v. 23, n. 9, p. 805-823, 1956.

TURNER, M.; DILL, E.; MARTIN, H. Large deflections of structures subjected to heating and external loads. Journal of the Aerospace Sciences, v. 26, n. 2, p. 97-106, 1960. 
VLADIMIROV, I. N.; PIETRYGA, M. P.; REESE, S. On the modelling of non-linear kinematic hardening at finite strains with application to springback - comparison of time integration algorithms. International Journal for Numerical Methods in Engineering, v. 75, n. 1, p. 1-28, 2007.

VLADIMIROV, I. N.; PIETRYGA, M. P.; REESE, S. Anisotropic finite elastoplasticity with nonlinear kinematic and isotropic hardening and application to sheet metal forming. International Journal of Plasticity, v. 26, n. 5, p. 659 - 687, 2010. ISSN 0749-6419.

WANG, W. M.; SLUYS, L. J.; BORST, R. de. Viscoplasticity for instabilities due to strain softening and strain-rate softening. International Journal for Numerical Methods in Engineering, v. 40, n. 20, p. 3839-3864, 1997.

WILLIAMS, T.; KELLEY, C.; many others. Gnuplot 4.6: an interactive plotting program. 2013. <http://gnuplot.sourceforge.net/>.

WRIGGERS, P. Computational Contact Mechanics. [S.l.]: Springer Berlin Heidelberg, 2006. ISBN 9783540326090.

WRIGGERS, P.; SIMO, J. C. A note on tangent stiffness for fully nonlinear contact problems. Communications in Applied Numerical Methods, John Wiley \& Sons, Ltd, v. 1, n. 5, p. 199-203, 1985. ISSN 1555-2047.

WRIGGERS, P.; VAN, T. V.; STEIN, E. Finite element formulation of large deformation impact-contact problems with friction. Computers and Structures, v. 37, n. 3, p. 319 331, 1990. ISSN 0045-7949.

YANG, B.; LAURSEN, T. A.; MENG, X. Two dimensional mortar contact methods for large deformation frictional sliding. International Journal for Numerical Methods in Engineering, v. 62, n. 9, p. 1183-1225, 2005.

YASTREBOV, V. Numerical Methods in Contact Mechanics. [S.l.]: Wiley, 2013. ISBN 9781848215191.

ZAVARISE, G.; LORENZIS, L. D. A modified node-to-segment algorithm passing the contact patch test. International Journal for Numerical Methods in Engineering, John Wiley \& Sons, Ltd., v. 79, n. 4, p. 379-416, 2009. ISSN 1097-0207.

ZHONG, Z.-H. Finite element procedures for contact-impact problems /

Zhi-Hua Zhong. [S.l.]: Oxford University Press Oxford ; New York, 1993. xi, 371 p. : p. ISBN 0198563833.

ZHONG, Z.-H.; NILSSON, L. A unified contact algorithm based on the territory concept. Computer Methods in Applied Mechanics and Engineering, v. 130, n. 1, p. 1 16, 1996. ISSN 0045-7825.

ZIENKIEWICZ, O. C.; TAYLOR, R. L. The finite element method for solid and structural mechanics. [S.l.]: Elsevier Butterworth-Heinemann, 2005. 733 p. ISBN 9780750664318. 


\section{Apêndice A}

\section{Álgebra tensorial}

Este apêndice tem como intuito apresentar, sem muito aprofundamento, alguns conceitos de álgebra tensorial fundamentais para o desenvolvimento do trabalho. Em geral, ao longo do texto, tenta-se ater à seguinte notação: escalares são simbolizados por letras em itálico (por exemplo, $t$ ou $T$ ); vetores ou tensores de primeira ordem por letras minúsculas em negrito (por exemplo, t); matrizes ou tensores de segunda ordem por letras maiúsculas em negrito (por exemplo, T); tensores de quarta ordem por letras em formato fraktur (por exemplo, T), com exceção do tensor identidade de quarta ordem, denotado por II. Quando utilizada a notação indicial para se referir a algum termo escalar dos tensores, este é alterado de negrito para itálico (por exemplo, $t_{i}$ ou $T_{i j}$ ).

\section{A.1 Contrações e produtos tensoriais}

A seguir são apresentadas algumas operações entre tensores comumente utilizadas ao longo do texto. Cada operação indicada é representada à direita em sua forma indicial, onde fica subentendido o somatório sobre os índices que não aparecem no outro lado da equação (chamados de índices mudos). Quando a forma indicial utiliza apenas um índice mudo, a operação é chamada de contração simples. Quando utilizam-se dois índices mudos, essa é chamada de contração dupla (denotada por :). Alguns exemplos de contrações são

$$
\begin{aligned}
a=\mathbf{b c} & \Longleftrightarrow a=b_{i} c_{i}, \\
\mathbf{C}=\mathbf{A B} & \Longleftrightarrow C_{i j}=A_{i k} B_{k j}, \\
\mathfrak{C}=\mathfrak{A B} & \Longleftrightarrow \mathfrak{C}_{i j k l}=\mathfrak{A}_{i m k l} B_{m j}, \\
\mathfrak{C}=\mathbf{A} \mathfrak{B} & \Longleftrightarrow \mathfrak{C}_{i j k l}=A_{i m} \mathfrak{B}_{m j k l}, \\
\mathfrak{D}=\mathbf{A} \mathfrak{B C} & \Longleftrightarrow \mathfrak{D}_{i j k l}=A_{i m} \mathfrak{B}_{m n k l} C_{n j}, \\
a=\mathbf{B}: \mathbf{C} & \Longleftrightarrow a=B_{i j} C_{i j}, \\
\mathbf{C}=\mathfrak{A}: \mathbf{B} & \Longleftrightarrow C_{i j}=\mathfrak{A}_{i j k l} B_{k l} \mathrm{e} \\
\mathfrak{C}=\mathfrak{A}: \mathfrak{B} & \Longleftrightarrow \mathfrak{C}_{i j k l}=\mathfrak{A}_{i j m n} \mathfrak{B}_{m n k l} .
\end{aligned}
$$

Devido ao elevado número de combinações de índices possíveis, as operações de 
contração podem variar a depender do caso, sendo assim preferível, sempre que possível, utilizar a notação indicial para indicar a operação que está sendo realizada.

Já a operação $\otimes$ denota o produto tensorial, e em geral não utiliza índices mudos. Alguns exemplos são

$$
\begin{aligned}
\mathbf{C}=\mathbf{a} \otimes \mathbf{b} & \Longleftrightarrow C_{i j}=a_{i} b_{j} \mathrm{e} \\
\mathfrak{C}=\mathbf{A} \otimes \mathbf{B} & \Longleftrightarrow \mathfrak{C}_{i j k l}=A_{i j} B_{k l} .
\end{aligned}
$$

\section{A.2 Tensores identidade}

Os tensores identidade de segunda e quarta ordem, denotados respectivamente por I e II, são definidos por

$$
\begin{aligned}
& I_{i j}=\delta_{i j}, \quad \mathrm{e} \\
& I I_{i j k l}=\delta_{i k} \delta_{j l},
\end{aligned}
$$

onde $\delta_{i j}$ é a função delta de Kronecker:

$$
\delta_{i j}=\left\{\begin{array}{l}
1, \text { se } i=j \\
0, \text { se } i \neq j .
\end{array}\right.
$$

\section{A.3 Tensor inverso de quarta ordem}

Seja $\mathbf{T}$ um tensor de segunda ordem. Sabe-se que o seu inverso é o tensor $\mathbf{T}^{-1}$ tal que

$$
\mathbf{T}^{-1} \mathbf{T}=\mathbf{T} \mathbf{T}^{-1}=\mathbf{I}
$$

Generalizando esse conceito para tensores de quarta ordem, temos que o inverso de $\mathfrak{T}$ é o tensor $\mathfrak{T}^{-1}$ tal que

$$
\mathfrak{T}^{-1}: \mathfrak{T}=\mathfrak{T}: \mathfrak{T}^{-1}=\mathbf{I I}
$$

Determinar o tensor inverso de quarta ordem é necessário em diversas passagens da formulação descrita, em especial no cálculo dos operadores tangentes consistentes visco-elástico e visco-elasto-plástico. Uma vez que a maioria dos pacotes computacionais básicos não realizam essa operação, utiliza-se neste trabalho um procedimento alternativo que consiste em representar o tensor $\mathfrak{T}$ de dimensões $n \times n \times n \times n$ em um tensor de segunda ordem com dimensões $n^{2} \times n^{2}$, e calcular a inversa desse.

\section{A.4 Derivadas tensoriais}

Nesta seção são enumeradas algumas derivadas de tensores recorrentes ao longo do texto, onde $\mathbf{T}$ é um tensor genérico de segunda ordem: 
- Derivada do quadrado de um tensor:

$$
\frac{\partial\left(T^{2}\right)_{i j}}{\partial T_{k l}}=\delta_{i k} T_{l j}+T_{i k} \delta_{j l}
$$

- Derivada da inversa de um tensor assimétrico:

$$
\frac{\partial T_{i j}^{-1}}{\partial T_{k l}}=-T_{i k}^{-1} T_{l j}^{-1}
$$

- Derivada da inversa de um tensor simétrico ${ }^{1}$ :

$$
\frac{\partial T_{i j}^{-1}}{\partial T_{k l}}=-\frac{1}{2}\left(T_{i k}^{-1} T_{j l}^{-1}+T_{i l}^{-1} T_{j k}^{-1}\right) .
$$

- Traço de um tensor (seção 2.3):

$$
\begin{gathered}
\operatorname{tr}(\mathbf{T})=\mathbf{I}: \mathbf{T}=T_{i i}, \\
\frac{\partial \operatorname{tr}(\mathbf{T})}{\partial T_{i j}}=\delta_{i j} .
\end{gathered}
$$

- Tensor desviador (seção 2.4):

$$
\begin{aligned}
& \mathbf{T}^{D}=\mathbf{T}-\frac{1}{3} \operatorname{tr}(\mathbf{T}) \mathbf{I}, \\
& \frac{\partial \mathbf{T}^{D}}{\partial \mathbf{T}}=\mathbf{I I}-\frac{1}{3} \mathbf{I} \otimes \mathbf{I} .
\end{aligned}
$$

- Derivada do determinante de um tensor:

$$
\begin{gathered}
\frac{\partial \operatorname{det}(\mathbf{T})}{\partial \mathbf{T}}=\operatorname{det}(\mathbf{T}) \mathbf{T}^{-T} \\
\frac{\partial \sqrt{\operatorname{det}(\mathbf{T})}}{\partial \mathbf{T}}=\frac{1}{2} \sqrt{\operatorname{det}(\mathbf{T})} \mathbf{T}^{-T}
\end{gathered}
$$

- Parcela simétrica de um tensor:

$$
\begin{aligned}
\operatorname{sim}(\mathbf{T}) & =\frac{1}{2}\left(\mathbf{T}+\mathbf{T}^{T}\right), \\
\frac{\partial \operatorname{sim}(\mathbf{T})}{\partial \mathbf{T}} & =\frac{1}{2}\left(\mathbf{I I}+\mathbf{I I}^{T}\right) .
\end{aligned}
$$

- Parcela antissimétrica de um tensor:

$$
\begin{gathered}
\operatorname{ant}(\mathbf{T})=\frac{1}{2}\left(\mathbf{T}-\mathbf{T}^{T}\right), \\
\frac{\partial \operatorname{ant}(\mathbf{T})}{\partial \mathbf{T}}=\frac{1}{2}\left(\mathbf{I I}-\mathbf{I I}^{T}\right) .
\end{gathered}
$$

- Norma de um tensor:

$$
\begin{aligned}
& \|\mathbf{T}\|=\sqrt{\mathbf{T}: \mathbf{T}}, \\
& \frac{\partial\|\mathbf{T}\|}{\partial \mathbf{T}}=\frac{\mathbf{T}}{\|\mathbf{T}\|} .
\end{aligned}
$$

$\overline{1}$ Essa expressão deve ser aplicada caso $\mathbf{T}$ seja representado apenas pela sua parte simétrica. 


\section{A.5 Relações tensoriais}

A seguir são apresentadas algumas relações tensoriais relevantes ao longo do texto, sendo a maioria dessas facilmente demonstradas utilizando a notação indicial:

$$
\begin{gathered}
(\mathbf{A}: \mathbf{B}) \mathbf{C}=(\mathbf{C} \otimes \mathbf{A}): \mathbf{B}=(\mathbf{C} \otimes \mathbf{B}): \mathbf{A}, \\
(\mathbf{A B}): \mathbf{C}=\mathbf{A}:\left(\mathbf{B}^{T} \mathbf{C}\right)=\mathbf{B}:\left(\mathbf{A}^{T} \mathbf{C}\right) \\
\mathbf{A}:(\mathbf{B C D})=\left(\mathbf{B}^{T} \mathbf{A D}^{T}\right): \mathbf{C} \\
\mathbf{A}:\left(\mathbf{B} \mathbf{C}^{T} \mathbf{D}\right)=\left(\mathbf{D} \mathbf{A}^{T} \mathbf{B}\right): \mathbf{C} \\
\mathbf{A}^{-T}:(\mathbf{B A})=\mathbf{I}: \mathbf{B}=\operatorname{tr}(\mathbf{B}) \\
\left(\mathbf{A}^{-1} \mathbf{B A}\right):\left(\mathbf{A}^{-1} \mathbf{B} \mathbf{A}\right)^{T}=\mathbf{B}: \mathbf{B}^{T} \\
(\mathbf{A}-\mathbf{B} \mathbf{A})^{D}=\mathbf{A}{ }^{-1} \mathbf{B}^{D} \mathbf{A} \\
\frac{\partial \mathbf{A}^{D}}{\partial \mathbf{A}}: \mathbf{B}=\mathbf{B}^{D} \\
\mathbf{A}: \mathbf{A}^{D}=\mathbf{A}^{D}: \mathbf{A}^{D} \\
\operatorname{sim}(\mathbf{A}): \mathbf{B}=\operatorname{sim}(\mathbf{A}): \operatorname{sim}(\mathbf{B})
\end{gathered}
$$




\section{Apêndice B}

\section{Solução dos algoritmos de retorno}

Este apêndice tem o intuito de detalhar o processo para resolução dos algoritmos de retorno dos modelos constitutivos apresentados, utilizando o método de Newton-Raphson ${ }^{1}$. Para facilitar a implementação computacional, as expressões são apresentadas em notação indicial na ordem em que devem ser calculadas, levando em conta as respectivas dependências. Além disso, para simplificar a notação, os índices $(\cdot)_{s+1}$ foram omitidos. Sendo assim, as variáveis sem índices devem ser consideradas no passo atual.

\section{B.1 Modelo elasto-plástico e visco-plástico}

Nos casos elasto-plástico e visco-plástico, deve-se resolver, respectivamente, os sistemas não-lineares (4.105) e (4.117). Seja $\{\mathbf{r}\}$ o vetor resíduo do sistema e $\{\mathbf{s}\}$ o vetor das variáveis do problema, arranjados na forma

$$
\{\mathbf{r}\}=\left\{\begin{array}{c}
\left\{\mathbf{R}_{1}\right\} \\
r_{2} \\
\left\{\mathbf{R}_{3}\right\} \\
r_{4}
\end{array}\right\} \quad \text { e } \quad\{\mathbf{s}\}=\left\{\begin{array}{c}
\left\{\mathbf{A}^{p}\right\} \\
\kappa \\
\{\mathbf{X}\} \\
\Delta \gamma
\end{array}\right\},
$$

onde $\left\{\mathbf{R}_{1}\right\},\left\{\mathbf{R}_{3}\right\},\left\{\mathbf{A}^{p}\right\}$ e $\{\mathbf{X}\}$ são representações vetoriais dos tensores de segunda ordem $\mathbf{R}_{1}, \mathbf{R}_{4}, \mathbf{A}^{p}$ e $\mathbf{X}$. Nesses, a seguinte ordenação dos índices pode ser adotada:

$$
\{(\cdot)\}^{T}=\left\{(\cdot)_{11} \quad(\cdot)_{12} \quad(\cdot)_{13} \quad(\cdot)_{21} \quad(\cdot)_{22} \quad(\cdot)_{23} \quad(\cdot)_{31} \quad(\cdot)_{32} \quad(\cdot)_{33}\right\} \cdot
$$

A linearização do sistema é realizada por meio da matriz Jacobiana $[\mathbf{J}]$, definida como

$$
[\mathbf{J}]=\frac{\partial\{\mathbf{r}\}}{\partial\{\mathbf{s}\}} \quad \text { ou } \quad[J]_{i j}=\frac{\partial\{r\}_{i}}{\partial\{s\}_{j}},
$$

e o erro é calculado por $\|\{\Delta \mathbf{s}\}\| /\|\{\mathbf{s}\}\|$, onde $\{\Delta \mathbf{s}\}$ é o vetor de correção aplicado em cada iteração.

1 Como o Método de Newton-Raphson já foi descrito com detalhes na seção 3.2, este apêndice usará uma abordagem direta, sem muitas explicações sobre a técnica propriamente dita. 
Parte-se das seguintes derivadas auxiliares:

$$
\begin{aligned}
& \frac{\partial C_{i j}^{e}}{\partial A_{k l}^{p}}=\underbrace{\frac{\partial\left(A^{p}\right)_{m i}^{-1}}{\partial A_{k l}^{p}}}_{\text {Eq.(A.17) }} C_{m n}\left(A^{p}\right)_{n j}^{-1}+\left(A^{p}\right)_{m i}^{-1} C_{m n} \underbrace{\frac{\left(A^{p}\right)_{n j}^{-1}}{\partial A_{k l}^{p}}}_{\text {Eq.(A.17) }}, \\
& \frac{\partial M_{i j}^{e}}{\partial A_{k l}^{p}}=\underbrace{\frac{\partial C_{i m}^{e}}{\partial A_{k l}^{p}}}_{\text {Eq.(B.4) }} S_{m j}^{e}+\frac{1}{2} C_{i m}^{e} \mathfrak{C}_{m j p q}^{e} \underbrace{\frac{\partial C_{p q}^{e}}{\partial A_{k l}^{p}}}_{\text {Eq.(B.4) }}, \\
& \frac{\partial\left(C_{e}^{p}\right)_{i j}}{\partial A_{k l}^{p}}=\left(A_{i}^{p}\right)_{l i}^{-1} A_{k m}^{p}\left(A_{i}^{p}\right)_{m j}^{-1}+\left(A_{i}^{p}\right)_{m i}^{-1} A_{k m}^{p}\left(A_{i}^{p}\right)_{l j}^{-1}, \\
& \frac{\partial\left(C_{e}^{p}\right)_{i j}}{\partial\left(A_{i}^{p}\right)_{k l}}=\underbrace{\frac{\partial\left(A_{i}^{p}\right)_{m i}^{-1}}{\partial\left(A_{i}^{p}\right)_{k l}}}_{\text {Eq.(A.17) }} A_{p m}^{p} A_{p n}^{p}\left(A_{i}^{p}\right)_{n j}^{-1}+\left(A_{i}^{p}\right)_{m i}^{-1} A_{p m}^{p} A_{p n}^{p} \underbrace{\frac{\left(A_{i}^{p}\right)_{n j}^{-1}}{\partial\left(A_{i}^{p}\right)_{k l}}}_{\text {Eq.(A.17) }}, \\
& \frac{\partial X_{i j}}{\partial A_{k l}^{p}}=2\left(\mathfrak{C}_{e}^{p}\right)_{i j m n} \underbrace{\frac{\partial\left(C_{e}^{p}\right)_{m n}}{\partial A_{k l}^{p}}}_{\text {Eq.(B.6) }}, \\
& \frac{\partial X_{i j}}{\partial\left(A_{i}^{p}\right)_{k l}}=2\left(\mathfrak{C}_{e}^{p}\right)_{i j m n} \underbrace{\frac{\partial\left(C_{e}^{p}\right)_{m n}}{\partial\left(A_{i}^{p}\right)_{k l}}}_{\text {Eq.(B.7) }}, \\
& \frac{\partial \chi_{i j}}{\partial A_{k l}^{p}}=\delta_{i k}\left(A_{i}^{p}\right)_{l m}^{-1} X_{m n}\left(A_{i}^{p}\right)_{p n}^{-1} A_{j p}^{p}+A_{i m}^{p}\left(A_{i}^{p}\right)_{m n}^{-1} X_{n p}\left(A_{i}^{p}\right)_{l p}^{-1} \delta_{j k} \\
& +A_{i m}^{p}\left(A_{i}^{p}\right)_{m n}^{-1} \underbrace{\frac{\partial X_{n p}}{\partial A_{k l}^{p}}}_{\text {Eq.(B.8) }}\left(A_{i}^{p}\right)_{q p}^{-1} A_{j q}^{p} \\
& \frac{\partial \chi_{i j}}{\partial\left(A_{i}^{p}\right)_{k l}}=A_{i m}^{p} \overbrace{\frac{\partial\left(A_{i}^{p}\right)_{m n}^{-1}}{\partial\left(A_{i}^{p}\right)_{k l}}}^{\text {Eq.(A.17) }} X_{n p}\left(A_{i}^{p}\right)_{q p}^{-1} A_{j q}^{p}+A_{i m}^{p}\left(A_{i}^{p}\right)_{m n}^{-1} X_{n p} \overbrace{\frac{\partial\left(A_{i}^{p}\right)_{q p}^{-1}}{\partial\left(A_{i}^{p}\right)_{k l}} A_{j q}^{p}}^{\text {Eq.(A.17) }} \\
& +A_{i m}^{p}\left(A_{i}^{p}\right)_{m n}^{-1} \underbrace{\frac{\partial(X)_{n p}}{\partial\left(A_{i}^{p}\right)_{k l}}}_{\text {Eq.(B.9) }}\left(A_{i}^{p}\right)_{q p}^{-1} A_{j q}^{p}, \\
& \frac{\partial\left(M_{e}^{p}\right)_{i j}}{\partial A_{k l}^{p}}=\underbrace{\frac{\partial\left(C_{e}^{p}\right)_{i m}}{\partial A_{k l}^{p}}}_{\text {Eq.(B.6) }} X_{m j}+\left(C_{e}^{p}\right)_{i m} \underbrace{\frac{\partial X_{m j}}{\partial A_{k l}^{p}}}_{\text {Eq.(B.8) }}, \\
& \frac{\partial\left(M_{e}^{p}\right)_{i j}}{\partial\left(A_{i}^{p}\right)_{k l}}=\underbrace{\frac{\partial\left(C_{e}^{p}\right)_{i m}}{\partial\left(A_{i}^{p}\right)_{k l}}}_{\text {Eq.(B.7) }} X_{m j}+\left(C_{e}^{p}\right)_{i m} \underbrace{\frac{\partial X_{m j}}{\partial\left(A_{i}^{p}\right)_{k l}}}_{\text {Eq.(B.9) }},
\end{aligned}
$$




$$
\begin{aligned}
& \frac{\partial N_{i j}^{p}}{\partial A_{k l}^{p}}=\frac{1}{\left\|\left(\mathbf{M}^{e}-\boldsymbol{\chi}\right)^{D}\right\|} \overbrace{\frac{\partial\left(M^{e}-\chi\right)_{i j}^{D}}{\partial\left(M^{e}-\chi\right)_{m n}}}^{\text {Eq.(A.22) }}(\overbrace{\frac{\partial M_{m n}^{e}}{\partial A_{k l}^{p}}}^{\text {Eq.(B.5) }}-\overbrace{\frac{\partial \chi_{m n}}{\partial A_{k l}^{p}}}^{\text {Eq.(B.10) }}) \\
& -\frac{\left(M^{e}-\chi\right)_{i j}^{D}}{\left\|\left(\mathbf{M}^{e}-\chi\right)^{D}\right\|^{2}} \frac{\left(M^{e}-\chi\right)_{m n}^{D}}{\left\|\left(\mathbf{M}^{e}-\chi\right)^{D}\right\|} \underbrace{\frac{\partial\left(M^{e}-\chi\right)_{m n}^{D}}{\partial\left(M^{e}-\chi\right)_{p q}}}_{\text {Eq.(A.22) }}(\underbrace{\frac{\partial M_{p q}^{e}}{\partial A_{k l}^{p}}}_{\text {Eq.(B.5) }}-\underbrace{\frac{\partial \chi_{p q}}{\partial A_{k l}^{p}}}_{\text {Eq.(B.10) }}), \\
& \frac{\partial N_{i j}^{p}}{\partial\left(A_{i}^{p}\right)_{k l}}=-\frac{1}{\left\|\left(\mathbf{M}^{e}-\boldsymbol{\chi}\right)^{D}\right\|} \overbrace{\frac{\partial\left(M^{e}-\chi\right)_{i j}^{D}}{\partial\left(M^{e}-\chi\right)_{m n}}}^{\text {Eq.(A.22) }} \frac{\overbrace{\frac{\partial \chi_{m n}}{\partial\left(A_{i}^{p}\right)_{k l}}}^{\text {Eq.(B.11) }}}{\partial M^{e}} \\
& +\frac{\left(M^{e}-\chi\right)_{i j}^{D}}{\left\|\left(\mathbf{M}^{e}-\chi\right)^{D}\right\|^{2}} \frac{\left(M^{e}-\chi\right)_{m n}^{D}}{\left\|\left(\mathbf{M}^{e}-\chi\right)^{D}\right\|} \underbrace{\frac{\partial\left(M^{e}-\chi\right)_{m n}^{D}}{\partial\left(M^{e}-\chi\right)_{p q}}}_{\text {Eq.(A.22) }} \underbrace{\frac{\partial \chi_{p q}}{\partial\left(A_{i}^{p}\right)_{k l}}}_{\text {Eq.(B.11) }},
\end{aligned}
$$

de onde calculam-se os termos da matriz Jacobiana $[\mathbf{J}]$ diretamente pelas expressões:

$$
\begin{aligned}
& \frac{\partial\left(R_{1}\right)_{i j}}{\partial A_{k l}^{p}}=I I_{i j k l}-\Delta \gamma(\underbrace{\frac{\partial N_{i m}^{p}}{\partial A_{k l}^{p}}}_{\text {Eq.(B.14) }} A_{m j}^{p}+N_{i k}^{p} \delta_{j l}), \\
& \frac{\partial\left(R_{1}\right)_{i j}}{\partial\left(A_{i}^{p}\right)_{k l}}=-\Delta \gamma \underbrace{\frac{\partial N_{i m}^{p}}{\partial\left(A_{i}^{p}\right)_{k l}}}_{\text {Eq.(B.15) }} A_{m j}^{p}, \\
& \frac{\partial\left(R_{1}\right)_{i j}}{\partial \kappa}=0, \\
& \frac{\partial\left(R_{1}\right)_{i j}}{\partial \Delta \gamma}=-N_{i m}^{p} A_{m j}^{p}, \\
& \frac{\partial r_{2}}{\partial A_{i j}^{p}}=0 \\
& \frac{\partial r_{2}}{\partial\left(A_{i}^{p}\right)_{i j}}=0, \\
& \frac{\partial r_{2}}{\partial \kappa}=1 \text {, } \\
& \frac{\partial r_{2}}{\partial \Delta \gamma}=-\sqrt{\frac{2}{3}}, \\
& \frac{\partial\left(R_{3}\right)_{i j}}{\partial A_{k l}^{p}}=-\Delta \gamma-\frac{b}{c} \underbrace{\frac{\partial\left(M_{e}^{p}\right)_{i m}^{D}}{\partial\left(M_{e}^{p}\right)_{p q}}}_{\text {Eq.(A.22) }} \underbrace{\frac{\partial\left(M_{e}^{p}\right)_{p q}}{\partial A_{k l}^{p}}}_{\text {Eq.(B.12) }}\left(A_{i}^{p}\right)_{m j}, \\
& \frac{\partial\left(R_{3}\right)_{i j}}{\partial\left(A_{i}^{p}\right)_{k l}}=I I_{i j k l}-\Delta \gamma \frac{b}{c} \underbrace{\frac{\partial\left(M_{e}^{p}\right)_{i m}^{D}}{\partial\left(M_{e}^{p}\right)_{p q}}}_{\text {Eq.(A.22) }} \underbrace{\frac{\partial\left(M_{e}^{p}\right)_{p q}}{\partial\left(A_{i}^{p}\right)_{k l}}}_{\text {Eq.(B.13) }}\left(A_{i}^{p}\right)_{m j}-\Delta \gamma \frac{b}{c}\left(M_{e}^{p}\right)_{i k}^{D} \delta_{j l}, \\
& \frac{\partial\left(R_{3}\right)_{i j}}{\partial \kappa}=0, \\
& \frac{\partial\left(R_{3}\right)_{i j}}{\partial \Delta \gamma}=-\frac{b}{c}\left(M_{e}^{p}\right)_{i m}^{D}\left(A_{i}^{p}\right)_{m j} .
\end{aligned}
$$


Para o caso elasto-plástico:

$$
\begin{aligned}
& \frac{\partial r_{4}}{\partial A_{i j}^{p}}=\frac{\partial \Phi}{\partial A_{i j}^{p}}=\frac{\left(M^{e}-\chi\right)_{m n}^{D}}{\left\|\left(\mathbf{M}^{e}-\chi\right)^{D}\right\|} \underbrace{\frac{\partial\left(M^{e}-\chi\right)_{m n}^{D}}{\partial\left(M^{e}-\chi\right)_{k l}}}_{\text {Eq.(A.22) }}(\underbrace{\frac{\partial M_{k l}^{e}}{\partial A_{i j}^{p}}}_{\text {Eq.(B.5) }}-\underbrace{\frac{\partial \chi_{k l}}{\partial A_{i j}^{p}}}_{\text {Eq.(B.10) }}), \\
& \frac{\partial r_{4}}{\partial \kappa}=\frac{\partial \Phi}{\partial \kappa}=-\sqrt{\frac{2}{3}} \underbrace{\frac{\partial \sigma_{\kappa}(\kappa)}{\partial \kappa}}_{\text {Tab.1 }}, \\
& \frac{\partial r_{4}}{\partial\left(A_{i}^{p}\right)_{i j}}=\frac{\partial \Phi}{\partial\left(A_{i}^{p}\right)_{i j}}=-\frac{\left(M^{e}-\chi\right)_{m n}^{D}}{\left\|\left(\mathbf{M}^{e}-\chi\right)^{D}\right\|} \underbrace{\frac{\partial\left(M^{e}-\chi\right)_{m n}^{D}}{\partial\left(M^{e}-\chi\right)_{k l}}}_{\text {Eq.(A.22) }} \underbrace{\frac{\partial\left(A_{i}^{p}\right)_{i j}}{\partial\left(\chi_{k l}\right.}}_{\text {Eq.(B.11) }}, \\
& \frac{\partial r_{4}}{\partial \Delta \gamma}=0 .
\end{aligned}
$$

Para o caso visco-plástico com modelo de Perzyna e lei de Norton:

$$
\begin{aligned}
\frac{\partial r_{4}}{\partial A_{i j}^{p}} & =-\frac{m}{\alpha} \frac{\Delta t}{\eta}\left(\frac{\Phi}{\alpha}\right)^{m-1} \underbrace{\frac{\partial \Phi}{\partial A_{i j}^{p}}}_{\text {Eq.(B.28) }}, \\
\frac{\partial r_{4}}{\partial \kappa} & =-\frac{m}{\alpha} \frac{\Delta t}{\eta}\left(\frac{\Phi}{\alpha}\right)^{m-1} \underbrace{\frac{\partial \Phi}{\partial \kappa}}_{\text {Eq.(B.29) }}, \\
\frac{\partial r_{4}}{\partial X_{i j}} & =-\frac{m}{\alpha} \frac{\Delta t}{\eta}\left(\frac{\Phi}{\alpha}\right)^{m-1} \underbrace{\frac{\partial \Phi}{\partial X_{i j}}}_{\text {Eq.(B.30) }}, \\
\frac{\partial r_{4}}{\partial \Delta \gamma} & =1 .
\end{aligned}
$$

\section{B.2 Modelo visco-elástico}

No caso visco-elástico, o sistema não-linear é dado pela Equação (5.29). Dessa forma, o vetor resíduo é o arranjo $\left\{\mathbf{R}^{v}\right\}$, e o vetor de variáveis do problema é $\left\{\mathbf{A}^{v}\right\}$. Os passos para calcular a matriz Jacobiana são dados pelas seguintes Equações:

$$
\begin{aligned}
\frac{\partial C_{i j}^{e}}{\partial A_{k l}^{v}} & =\underbrace{\frac{\partial\left(A^{v}\right)_{m i}^{-1}}{\partial A_{k l}^{v}}}_{\text {Eq.(A.17) }} C_{m n}\left(A^{v}\right)_{n j}^{-1}+\left(A^{v}\right)_{m i}^{-1} C_{m n} \underbrace{\frac{\partial\left(A^{v}\right)_{n j}^{-1}}{\partial A_{k l}^{v}}}_{\text {Eq.(A.17) }} \\
\frac{\partial M_{i j}^{e}}{\partial A_{k l}^{v}} & =\underbrace{\frac{\partial C_{i m}^{e}}{\partial A_{k l}^{v}}}_{\text {Eq.(B.36) }} S_{m j}^{e}+\frac{1}{2} C_{i m}^{e} \mathfrak{C}_{m j p q}^{e} \underbrace{\frac{\partial C_{p q}^{e}}{\partial A_{k l}^{v}}}_{\text {Eq.(B.36) }}, \\
\frac{\partial R_{i j}^{v}}{\partial A_{k l}^{v}} & =I I_{i j k l}-\frac{\Delta t}{\eta} \underbrace{\frac{\partial\left(M^{e}\right)_{i m}^{D}}{\partial M_{n p}^{e}}}_{\text {Eq.(A.22) }} \underbrace{\frac{\partial M_{n p}^{e}}{\partial A_{k l}^{v}}}_{\text {Eq.(B.37) }} A_{m j}^{v}-\frac{\Delta t}{\eta}\left(M^{e}\right)_{i k}^{D} \delta_{j l} .
\end{aligned}
$$




\section{B.3 Modelo visco-elasto-plástico}

O modelo visco-elasto-plástico possui dois algoritmos de retorno: o primeiro para calcular a previsão visco-elástica da deformação, e o segundo para realizar a correção plástica.

\section{B.3.1 Previsão visco-elástica}

Neste caso, o sistema não-linear será igual ao apresentado na seção B.2, com a diferença que o valor de $\mathbf{C}^{e}$ será função também das deformações plásticas decorrentes de passos anteriores, conforme a Equação (6.8). Sendo assim, a Equação (B.36) deve ser substituída por

$$
\frac{\partial C_{i j}^{e}}{\partial A_{k l}^{v}}=\underbrace{\frac{\partial\left(A^{v}\right)_{m i}^{-1}}{\partial A_{k l}^{v}}}_{\text {Eq.(A.17) }}\left(A^{p}\right)_{n m}^{-1} C_{n p}\left(A^{p}\right)_{p q}^{-1}\left(A^{v}\right)_{q j}^{-1}+\left(A^{v}\right)_{m i}^{-1}\left(A^{p}\right)_{n m}^{-1} C_{n p}\left(A^{p}\right)_{p q}^{-1} \underbrace{\frac{\partial\left(A^{v}\right)_{q j}^{-1}}{\partial A_{k l}^{v}}}_{\text {Eq.(A.17) }} .
$$

Já as derivadas $\partial \mathbf{M}^{e} / \partial \mathbf{A}^{v}$ e $\partial \mathbf{R}^{v} / \partial \mathbf{A}^{v}$ podem ser calculadas ainda pelas Equações (B.37) e (B.38), respectivamente.

\section{B.3.2 Correção plástica}

Neste caso, o sistema não linear é dado por (6.39). O vetor resíduo do problema e o vetor de variáveis são, respectivamente,

$$
\{\mathbf{r}\}=\left\{\begin{array}{c}
\left\{\mathbf{R}^{v}\right\} \\
\left\{\mathbf{R}_{1}\right\} \\
r_{2} \\
\left\{\mathbf{R}_{3}\right\} \\
r_{4}
\end{array}\right\} \quad \text { e } \quad\{\mathbf{s}\}=\left\{\begin{array}{c}
\left\{\mathbf{A}^{v}\right\} \\
\left\{\mathbf{A}^{p}\right\} \\
\kappa \\
\{\mathbf{X}\} \\
\Delta \gamma
\end{array}\right\} .
$$

Parte-se das derivadas auxiliares:

$$
\begin{aligned}
& \frac{\partial C_{i j}^{v e}}{\partial A_{k l}^{p}}=\underbrace{\frac{\partial\left(A^{p}\right)_{m i}^{-1}}{\partial A_{k l}^{p}}}_{\text {Eq.(A.17) }} C_{m n}\left(A^{p}\right)_{n j}^{-1}+\left(A^{p}\right)_{m i}^{-1} C_{m n} \underbrace{\frac{\left(A^{p}\right)_{n j}^{-1}}{\partial A_{k l}^{p}}}_{\text {Eq.(A.17) }}, \\
& \frac{\partial M_{i j}^{\infty}}{\partial A_{k l}^{p}}=\underbrace{\frac{\partial C_{i m}^{v e}}{\partial A_{k l}^{p}}}_{\text {Eq.(B.41) }} S_{m j}^{\infty}+\frac{1}{2} C_{i p}^{v e} \mathfrak{C}_{p j m n}^{\infty} \underbrace{\frac{\partial C_{m n}^{v e}}{\partial A_{k l}^{p}}}_{\text {Eq.(B.41) }}, \\
& \frac{\partial C_{i j}^{e}}{\partial A_{k l}^{p}}=\left(A^{v}\right)_{m i}^{-1} \underbrace{\frac{\partial\left(A^{p}\right)_{n m}^{-1}}{\partial A_{k l}^{p}}}_{\text {Eq.(A.17) }} C_{n p}\left(A^{p}\right)_{p q}^{-1}\left(A^{v}\right)_{q j}^{-1}+\left(A^{v}\right)_{m i}^{-1}\left(A^{p}\right)_{n m}^{-1} C_{n p} \underbrace{\frac{\partial\left(A^{p}\right)_{p q}^{-1}}{\partial A_{k l}^{p}}}_{\text {Eq.(A.17) }}\left(A^{v}\right)_{q j}^{-1}, \\
& \frac{\partial C_{i j}^{e}}{\partial A_{k l}^{v}}=\underbrace{\frac{\partial\left(A^{v}\right)_{m i}^{-1}}{\partial A_{k l}^{v}}}_{\text {Eq.(A.17) }}\left(A^{p}\right)_{n m}^{-1} C_{n p}\left(A^{p}\right)_{p q}^{-1}\left(A^{v}\right)_{q j}^{-1}+\left(A^{v}\right)_{m i}^{-1}\left(A^{p}\right)_{n m}^{-1} C_{n p}\left(A^{p}\right)_{p q}^{-1} \underbrace{\frac{\partial\left(A^{v}\right)_{q j}^{-1}}{\partial A_{k l}^{v}}}_{\text {Eq.(A.17) }},
\end{aligned}
$$




$$
\begin{aligned}
& \frac{\partial M_{i j}^{e}}{\partial A_{k l}^{p}}=\underbrace{\frac{\partial C_{i m}^{e}}{\partial A_{k l}^{p}}}_{\text {Eq.(B.43) }} S_{m j}^{e}+\frac{1}{2} C_{i m}^{e} \mathfrak{C}_{m j p q}^{e} \underbrace{\frac{\partial C_{p q}^{e}}{\partial A_{k l}^{p}}}_{\text {Eq.(B.43) }}, \\
& \frac{\partial M_{i j}^{e}}{\partial A_{k l}^{v}}=\underbrace{\frac{\partial C_{i m}^{e}}{\partial A_{k l}^{v}}}_{\text {Eq.(B.44) }} S_{m j}^{e}+\frac{1}{2} C_{i m}^{e} \mathfrak{C}_{m j p q}^{e} \underbrace{\frac{\partial C_{p q}^{e}}{\partial A_{k l}^{v}}}_{\text {Eq.(B.44) }}, \\
& \frac{\partial M_{i j}^{q}}{\partial A_{k l}^{p}}=A_{m i}^{v} \underbrace{\frac{\partial M_{m n}^{e}}{\partial A_{k l}^{p}}}_{\text {Eq.(B.45) }}\left(A^{v}\right)_{j n}^{-1}, \\
& \frac{\partial M_{i j}^{q}}{\partial A_{k l}^{v}}=\delta_{i l} M_{k m}^{e}\left(A^{v}\right)_{j m}^{-1}+A_{m i}^{v} \underbrace{\frac{\partial M_{m n}^{e}}{\partial A_{k l}^{v}}}_{\text {Eq.(B.46) }}\left(A^{v}\right)_{j n}^{-1}+A_{m i}^{v} M_{m n}^{e} \underbrace{\frac{\partial\left(A^{v}\right)_{j n}^{-1}}{\partial A_{k l}^{v}}}_{\text {Eq.(A.17) }}, \\
& \frac{\partial\left(C_{e}^{p}\right)_{i j}}{\partial A_{k l}^{p}}=\left(A_{i}^{p}\right)_{l i}^{-1} A_{k m}^{p}\left(A_{i}^{p}\right)_{m j}^{-1}+\left(A_{i}^{p}\right)_{m i}^{-1} A_{k m}^{p}\left(A_{i}^{p}\right)_{l j}^{-1}, \\
& \frac{\partial\left(C_{e}^{p}\right)_{i j}}{\partial\left(A_{i}^{p}\right)_{k l}}=\underbrace{\frac{\partial\left(A_{i}^{p}\right)_{m i}^{-1}}{\partial\left(A_{i}^{p}\right)_{k l}}}_{\text {Eq.(A.17) }} A_{p m}^{p} A_{p n}^{p}\left(A_{i}^{p}\right)_{n j}^{-1}+\left(A_{i}^{p}\right)_{m i}^{-1} A_{p m}^{p} A_{p n}^{p} \underbrace{\frac{\left(A_{i}^{p}\right)_{n j}^{-1}}{\partial\left(A_{i}^{p}\right)_{k l}}}_{\text {Eq.(A.17) }}, \\
& \frac{\partial X_{i j}}{\partial A_{k l}^{p}}=2\left(\mathfrak{C}_{e}^{p}\right)_{i j m n} \underbrace{\frac{\partial\left(C_{e}^{p}\right)_{m n}}{\partial A_{k l}^{p}}}_{\text {Eq.(B.49) }}, \\
& \frac{\partial X_{i j}}{\partial\left(A_{i}^{p}\right)_{k l}}=2\left(\mathfrak{C}_{e}^{p}\right)_{i j m n} \underbrace{\frac{\partial\left(C_{e}^{p}\right)_{m n}}{\partial\left(A_{i}^{p}\right)_{k l}}}_{\text {Eq. (B.50) }}, \\
& \frac{\partial \chi_{i j}}{\partial A_{k l}^{p}}=\delta_{i k}\left(A_{i}^{p}\right)_{l m}^{-1} X_{m n}\left(A_{i}^{p}\right)_{p n}^{-1} A_{j p}^{p}+A_{i m}^{p}\left(A_{i}^{p}\right)_{m n}^{-1} X_{n p}\left(A_{i}^{p}\right)_{l p}^{-1} \delta_{j k} \\
& +A_{i m}^{p}\left(A_{i}^{p}\right)_{m n}^{-1} \underbrace{\frac{\partial X_{n p}}{\partial A_{k l}^{p}}}_{\text {Eq.(B.51) }}\left(A_{i}^{p}\right)_{q p}^{-1} A_{j q}^{p} \\
& \frac{\partial \chi_{i j}}{\partial\left(A_{i}^{p}\right)_{k l}}=A_{i m}^{p} \overbrace{\frac{\partial\left(A_{i}^{p}\right)_{m n}^{-1}}{\partial\left(A_{i}^{p}\right)_{k l}}}^{\text {Eq.(A.17) }} X_{n p}\left(A_{i}^{p}\right)_{q p}^{-1} A_{j q}^{p}+A_{i m}^{p}\left(A_{i}^{p}\right)_{m n}^{-1} X_{n p} \overbrace{\frac{\partial\left(A_{i}^{p}\right)_{q p}^{-1}}{\partial\left(A_{i}^{p}\right)_{k l}}}^{\text {Eq.(A.17) }} A_{j q}^{p} \\
& +A_{i m}^{p}\left(A_{i}^{p}\right)_{m n}^{-1} \underbrace{\frac{\partial(X)_{n p}}{\partial\left(A_{i}^{p}\right)_{k l}}}_{\text {Eq. (B.52) }}\left(A_{i}^{p}\right)_{q p}^{-1} A_{j q}^{p}, \\
& \frac{\partial\left(M_{e}^{p}\right)_{i j}}{\partial A_{k l}^{p}}=\underbrace{\frac{\partial\left(C_{e}^{p}\right)_{i m}}{\partial A_{k l}^{p}}}_{\text {Eq.(B.49) }} X_{m j}+\left(C_{e}^{p}\right)_{i m} \underbrace{\frac{\partial X_{m j}}{\partial A_{k l}^{p}}}_{\text {Eq.(B.51) }}, \\
& \frac{\partial\left(M_{e}^{p}\right)_{i j}}{\partial\left(A_{i}^{p}\right)_{k l}}=\underbrace{\frac{\partial\left(C_{e}^{p}\right)_{i m}}{\partial\left(A_{i}^{p}\right)_{k l}}}_{\text {Eq.(B.50) }} X_{m j}+\left(C_{e}^{p}\right)_{i m} \underbrace{\frac{\partial X_{m j}}{\partial\left(A_{i}^{p}\right)_{k l}}}_{\text {Eq.(B.52) }},
\end{aligned}
$$




$$
\begin{aligned}
& \frac{\partial N_{i j}^{p}}{\partial A_{k l}^{p}}=\frac{1}{\left\|\Sigma^{D}\right\|} \overbrace{\frac{\partial \Sigma_{i j}^{D}}{\partial \Sigma_{m n}}}^{\text {Eq.(A.22) }}(\overbrace{\frac{\partial M_{m n}^{\infty}}{\partial A_{k l}^{p}}}^{\text {Eq.(B.42) }}+\overbrace{\frac{\partial M_{m n}^{q}}{\partial A_{k l}^{p}}}^{\text {Eq.(B.47) }}-\overbrace{\frac{\partial \chi_{m n}}{\partial A_{k l}^{p}}}^{\text {Eq.(B.53) }}) \\
& -\frac{\Sigma_{i j}^{D}}{\left\|\boldsymbol{\Sigma}^{D}\right\|^{2}} \frac{\Sigma_{m n}^{D}}{\left\|\boldsymbol{\Sigma}^{D}\right\|} \underbrace{\frac{\partial \Sigma_{m n}^{D}}{\partial \Sigma_{p q}}}_{\text {Eq.(A.22) }}(\underbrace{\frac{\partial M_{p q}^{\infty}}{\partial A_{k l}^{p}}}_{\text {Eq.(B.42) }}+\underbrace{\frac{\partial M_{p q}^{q}}{\partial A_{k l}^{p}}}_{\text {Eq.(B.47) }}-\underbrace{\frac{\partial \chi_{p q}}{\partial A_{k l}^{p}}}_{\text {Eq.(B.53) }}), \\
& \frac{\partial N_{i j}^{p}}{\partial A_{k l}^{v}}=\frac{1}{\left\|\boldsymbol{\Sigma}^{D}\right\|} \underbrace{\frac{\partial \Sigma_{i j}^{D}}{\partial \Sigma_{m n}}}_{\text {Eq.(A.22) }} \underbrace{\frac{\partial M_{m n}^{q}}{\partial A_{k l}^{v}}}_{\text {Eq.(B.48) }}-\frac{\Sigma_{i j}^{D}}{\left\|\Sigma^{D}\right\|^{2}} \frac{\Sigma_{m n}^{D}}{\left\|\boldsymbol{\Sigma}^{D}\right\|} \underbrace{\frac{\partial \Sigma_{m n}^{D}}{\partial \Sigma_{p q}}}_{\text {Eq.(A.22) }} \underbrace{\frac{\partial M_{p q}^{q}}{\partial A_{k l}^{v}}}_{\text {Eq.(B.48) }}, \\
& \frac{\partial N_{i j}^{p}}{\partial\left(A_{i}^{p}\right)_{k l}}=-\frac{1}{\left\|\boldsymbol{\Sigma}^{D}\right\|} \underbrace{\frac{\partial \Sigma_{i j}^{D}}{\partial \Sigma_{m n}}}_{\text {Eq.(A.22) }} \underbrace{\frac{\partial \chi_{m n}}{\partial\left(A_{i}^{p}\right)_{k l}}}_{\text {Eq.(B.54) }}+\frac{\Sigma_{i j}^{D}}{\left\|\boldsymbol{\Sigma}^{D}\right\|^{2}} \frac{\Sigma_{m n}^{D}}{\left\|\boldsymbol{\Sigma}^{D}\right\|} \underbrace{\frac{\partial \Sigma_{m n}^{D}}{\partial \Sigma_{p q}}}_{\text {Eq.(A.22) }} \underbrace{\frac{\partial \chi_{p q}}{\partial\left(A_{i}^{p}\right)_{k l}}}_{\text {Eq.(B.54) }},
\end{aligned}
$$

de onde calculam-se os termos da matriz Jacobiana $[\mathbf{J}]$ diretamente pelas expressões:

$$
\begin{aligned}
& \frac{\partial R_{i j}^{v}}{\partial A_{k l}^{v}}=I I_{i j k l}-\frac{\Delta t}{\eta} \underbrace{\frac{\partial\left(M^{e}\right)_{i m}^{D}}{\partial M_{n p}^{e}}}_{\text {Eq.(A.22) }} \underbrace{\frac{\partial M_{n p}^{e}}{\partial A_{k l}^{v}}}_{\text {Eq.(B.37) }} A_{m j}^{v}-\frac{\Delta t}{\eta}\left(M^{e}\right)_{i k}^{D} \delta_{j l}, \\
& \frac{\partial R_{i j}^{v}}{\partial A_{k l}^{p}}=-\frac{\Delta t}{\eta} \underbrace{\frac{\partial\left(M^{e}\right)_{i p}^{D}}{\partial M_{m n}^{e}}}_{\text {Eq.(A.22) }} \underbrace{\frac{\partial M_{m n}^{e}}{\partial A_{k l}^{p}}}_{\text {Eq.(B.45) }} A_{p j}^{v}, \\
& \frac{\partial R_{i j}^{v}}{\partial \kappa}=\frac{\partial R_{i j}^{v}}{\partial\left(A_{i}^{p}\right)_{k l}}=\frac{\partial R_{i j}^{v}}{\partial \Delta \gamma}=0, \\
& \frac{\partial\left(R_{1}\right)_{i j}}{\partial A_{k l}^{v}}=-\Delta \gamma \underbrace{\frac{\partial N_{i m}^{p}}{\partial A_{k l}^{v}}}_{\text {Eq.(B.58) }} A_{m j}^{p}, \\
& \frac{\partial\left(R_{1}\right)_{i j}}{\partial A_{k l}^{p}}=I I_{i j k l}-\Delta \gamma(\underbrace{\frac{\partial N_{i m}^{p}}{\partial A_{k l}^{p}}}_{\text {Eq.(B.14) }} A_{m j}^{p}+N_{i k}^{p} \delta_{j l}), \\
& \frac{\partial\left(R_{1}\right)_{i j}}{\partial\left(A_{i}^{p}\right)_{k l}}=-\Delta \gamma \underbrace{\frac{\partial N_{i m}^{p}}{\partial\left(A_{i}^{p}\right)_{k l}}}_{\text {Eq.(B.15) }} A_{m j}^{p}, \\
& \frac{\partial\left(R_{1}\right)_{i j}}{\partial \kappa}=0, \\
& \frac{\partial\left(R_{1}\right)_{i j}}{\partial \Delta \gamma}=-N_{i m}^{p} A_{m j}^{p}, \\
& \frac{\partial r_{2}}{\partial A_{i j}^{v}}=\frac{\partial r_{2}}{\partial A_{i j}^{p}}=\frac{\partial r_{2}}{\partial\left(A_{i}^{p}\right)_{i j}}=0, \\
& \frac{\partial r_{2}}{\partial \kappa}=1 \text {, } \\
& \frac{\partial r_{2}}{\partial \Delta \gamma}=-\sqrt{\frac{2}{3}},
\end{aligned}
$$




$$
\begin{aligned}
& \frac{\partial\left(R_{3}\right)_{i j}}{\partial A_{k l}^{v}}=\frac{\partial\left(R_{3}\right)_{i j}}{\partial \kappa}=0, \\
& \frac{\partial\left(R_{3}\right)_{i j}}{\partial A_{k l}^{p}}=-\Delta \gamma-\frac{b}{c} \underbrace{\frac{\partial\left(M_{e}^{p}\right)_{i m}^{D}}{\partial\left(M_{e}^{p}\right)_{p q}} \underbrace{\frac{\partial\left(M_{e}^{p}\right)_{p q}}{\partial A_{k l}^{p}}}_{\text {Eq.(B.12) }}\left(A_{i}^{p}\right)_{m j},}_{\text {Eq.(A.22) }} \\
& \frac{\partial\left(R_{3}\right)_{i j}}{\partial\left(A_{i}^{p}\right)_{k l}}=I I_{i j k l}-\Delta \gamma \frac{b}{c} \underbrace{\frac{\partial\left(M_{e}^{p}\right)_{i m}^{D}}{\partial\left(M_{e}^{p}\right)_{p q}}}_{\text {Eq.(A.22) }} \underbrace{\frac{\partial\left(M_{e}^{p}\right)_{p q}}{\partial\left(A_{i}^{p}\right)_{k l}}}_{\text {Eq.(B.13) }}\left(A_{i}^{p}\right)_{m j}-\Delta \gamma-\left(M_{e}^{p}\right)_{i k}^{D} \delta_{j l}, \\
& \frac{\partial\left(R_{3}\right)_{i j}}{\partial \Delta \gamma}=-\frac{b}{c}\left(M_{e}^{p}\right)_{i m}^{D}\left(A_{i}^{p}\right)_{m j} .
\end{aligned}
$$

Para o caso visco-elasto-plástico:

$$
\begin{aligned}
& \frac{\partial r_{4}}{\partial A_{i j}^{v}}=\frac{\partial \Phi}{\partial A_{i j}^{v}}=\frac{\Sigma_{m n}^{D}}{\left\|\Sigma^{D}\right\|} \underbrace{\frac{\partial \Sigma_{m n}^{D}}{\partial \Sigma_{k l}}}_{\text {Eq.(A.22) }} \underbrace{\frac{\partial M_{k l}^{q}}{\partial A_{i j}^{v}}}_{\text {Eq.(B.48) }}, \\
& \frac{\partial r_{4}}{\partial A_{i j}^{p}}=\frac{\partial \Phi}{\partial A_{i j}^{p}}=\frac{\Sigma_{m n}^{D}}{\left\|\Sigma^{D}\right\|} \underbrace{\frac{\partial \Sigma_{m n}^{D}}{\partial \Sigma_{k l}}}_{\text {Eq.(A.22) }}(\underbrace{\frac{\partial M_{k l}^{\infty}}{\partial A_{i j}^{p}}}_{\text {Eq.(B.42) }}+\underbrace{\frac{\partial M_{k l}^{q}}{\partial A_{i j}^{p}}}_{\text {Eq.(B.47) }}-\underbrace{\frac{\partial \chi_{k l}}{\partial A_{i j}^{p}}}_{\text {Eq.(B.53) }}), \\
& \frac{\partial r_{4}}{\partial \kappa}=\frac{\partial \Phi}{\partial \kappa}=-\sqrt{\frac{2}{3}} \underbrace{\frac{\partial \sigma_{\kappa}(\kappa)}{\partial \kappa}}_{\text {Tab.1 }}, \\
& \frac{\partial r_{4}}{\partial\left(A_{i}^{p}\right)_{i j}}=\frac{\partial \Phi}{\partial\left(A_{i}^{p}\right)_{i j}}=-\frac{\Sigma_{m n}^{D}}{\left\|\Sigma^{D}\right\|} \underbrace{\frac{\partial \Sigma_{m n}^{D}}{\partial \Sigma_{k l}}}_{\text {Eq.(A.22) }} \underbrace{\frac{\partial \chi_{k l}}{\partial\left(A_{i}^{p}\right)_{i j}}}_{\text {Eq.(B.54) }}, \\
& \frac{\partial r_{4}}{\partial \Delta \gamma}=0 .
\end{aligned}
$$

Para o caso visco-elasto-visco-plástico com modelo de Perzyna e lei de Norton:

$$
\begin{aligned}
& \frac{\partial r_{4}}{\partial A_{i j}^{v}}=-\frac{m}{\alpha} \frac{\Delta t}{\eta}\left(\frac{\Phi}{\alpha}\right)^{m-1} \underbrace{\frac{\partial \Phi}{\partial A_{i j}^{v}}}_{\text {Eq.(B.75) }}, \\
& \frac{\partial r_{4}}{\partial A_{i j}^{p}}=-\frac{m}{\alpha} \frac{\Delta t}{\eta}\left(\frac{\Phi}{\alpha}\right)^{m-1} \underbrace{\frac{\partial \Phi}{\partial A_{i j}^{p}}}_{\text {Eq.(B.76) }}, \\
& \frac{\partial r_{4}}{\partial \kappa}=-\frac{m}{\alpha} \frac{\Delta t}{\eta}\left(\frac{\Phi}{\alpha}\right)^{m-1} \underbrace{\frac{\partial \Phi}{\partial \kappa}}_{\text {Eq.(B.77) }}, \\
& \frac{\partial r_{4}}{\partial\left(A_{i}^{p}\right)_{i j}}=-\frac{m}{\alpha} \frac{\Delta t}{\eta}\left(\frac{\Phi}{\alpha}\right)^{m-1} \underbrace{\frac{\partial \Phi}{\partial\left(A_{i}^{p}\right)_{i j}}}_{\text {Eq.(B.78) }} \\
& \frac{\partial r_{4}}{\partial \Delta \gamma}=1 .
\end{aligned}
$$




\section{Apêndice C}

\section{Cálculo do operador tangente consistente}

Para facilitar a implementação computacional, as expressões a seguir são apresentadas em notação indicial na ordem em que devem ser calculadas, levando em conta as respectivas dependências.

\section{C.1 Modelo elasto-plástico e visco-plástico}

No modelo elasto-plástico/visco-plástico, deve-se distinguir o operador tangente consistente entre os casos onde ocorre plastificação $(\Delta \gamma>0)$ e onde não ocorre $(\Delta \gamma=0)$. Nesta seção apresenta-se o cálculo de ambos os casos.

\section{C.1.1 Caso não ocorra plastificação}

Neste caso, segue de imediato

$\frac{d \mathbf{A}^{p}}{d \mathbf{E}}=\frac{d\left(\mathbf{A}^{p}\right)^{-1}}{d \mathbf{E}}=\mathbf{0}$

Logo:

$\frac{d S_{i j}^{e}}{d E_{k l}}=\mathfrak{C}_{i j m n}^{e} \frac{d C_{m n}^{e}}{d C_{k l}}=\mathfrak{C}_{i j m n}^{e}\left(A^{p}\right)_{k m}^{-1}\left(A^{p}\right)_{l n}^{-1}$,

e a Equação (4.95) pode ser simplificada para

$\mathfrak{C}_{i j k l}^{e p}=\left(A^{p}\right)_{i m}^{-1} \frac{d S_{m n}^{e}}{d E_{k l}}\left(A^{p}\right)_{j n}^{-1}=\left(A^{p}\right)_{i m}^{-1} \mathfrak{C}_{m n p q}^{e}\left(A^{p}\right)_{k p}^{-1}\left(A^{p}\right)_{l q}^{-1}\left(A^{p}\right)_{j n}^{-1}$.

Observa-se que, caso não tenha ocorrido plastificação em nenhum passo anterior (ou seja, $\mathbf{A}^{p}=\mathbf{I}$ ), a expressão (C.3) reduz-se a $\mathfrak{C}^{e p}=\mathfrak{C}^{e}$, como esperado.

\section{C.1.2 Caso ocorra plastificação}

Neste caso, temos:

$\frac{\partial C_{i j}^{e}}{\partial E_{k l}}=2\left(A^{p}\right)_{k i}^{-1}\left(A^{p}\right)_{l j}^{-1}$ 


$$
\begin{aligned}
& \frac{\partial M_{i j}^{e}}{\partial E_{k l}}=\underbrace{\frac{\partial C_{i p}^{e}}{\partial E_{k l}}}_{\text {Eq.(C.4) }} S_{p j}^{e}+\frac{1}{2} C_{i p}^{e} \mathfrak{C}_{p j m n}^{e} \underbrace{\frac{\partial C_{m n}^{e}}{\partial E_{k l}}}_{\text {Eq.(C.4) }}, \\
& \frac{\partial \Phi}{\partial E_{i j}}=\frac{\left(M^{e}-\chi\right)_{k l}^{D}}{\left\|\left(\mathbf{M}^{e}-\chi\right)^{D}\right\|} \underbrace{\frac{\partial\left(M^{e}-\chi\right)_{k l}^{D}}{\partial\left(M^{e}-\chi\right)_{m n}}}_{\text {Eq.(A.22) }} \underbrace{\frac{\partial M_{m n}^{e}}{\partial E_{i j}}}_{\text {Eq.(C.5) }},
\end{aligned}
$$

No caso elasto-plástico, utiliza-se a Equação (4.98) para o cálculo de $\mathbf{D}^{\gamma}$ :

$$
D_{i j}^{\gamma}=-\frac{\overbrace{\partial \Phi / \partial E_{i j}}^{\text {Eq.(C.6) }}}{\underbrace{\frac{\partial \Phi}{\partial A_{k l}^{p}}}_{\text {Eq.(B.28) }} N_{k m}^{p} A_{m l}^{p}+\underbrace{\frac{\partial \Phi}{\partial\left(A_{i}^{p}\right)_{k l}}}_{\text {Eq.(B.30) }}\left(N_{i}^{p}\right)_{k m}\left(A_{i}^{p}\right)_{m l}+\underbrace{\frac{\partial \Phi}{\partial \kappa}}_{\text {Eq.(B.29) }} \sqrt{\frac{2}{3}}},
$$

onde os índices $k, l$ e $m$ devem ser somados dentro do denominador. Já no caso visco-plástico com modelo de Perzyna, utiliza-se a Equação (4.120):

$D_{i j}^{\gamma}=-\frac{\partial r_{\gamma} / \partial E_{i j}}{\frac{\partial r_{\gamma}}{\partial A_{k l}^{p}} N_{k m}^{p} A_{m l}^{p}+\frac{\partial r_{\gamma}}{\partial\left(A_{i}^{p}\right)_{k l}}\left(N_{i}^{p}\right)_{k m}\left(A_{i}^{p}\right)_{m l}+\frac{\partial r_{\gamma}}{\partial \kappa} \sqrt{\frac{2}{3}}+1}$,

onde $\partial r_{\gamma} / \partial \mathbf{E}, \partial r_{\gamma} / \partial \mathbf{A}^{p}, \partial r_{\gamma} / \partial \mathbf{A}_{i}^{p}$ e $\partial r_{\gamma} / \partial \kappa$ podem ser calculados conforme a Equação (4.121) quando utilizada a lei de Norton. Em seguida, pela Equação (4.100),

$\frac{d A_{i j}^{p}}{d E_{k l}}=N_{i m}^{p} A_{m j}^{p} D_{k l}^{\gamma}$

de onde resulta

$\frac{d\left(A^{p}\right)_{i j}^{-1}}{d E_{k l}}=\underbrace{\frac{d\left(A^{p}\right)_{i j}^{-1}}{d A_{m n}^{p}}}_{\text {Eq.(A.17) }} \underbrace{\frac{d A_{m n}^{p}}{d E_{k l}}}_{\text {Eq.(C.9) }}$,

$\frac{d S_{i j}^{e}}{d E_{k l}}=\frac{1}{2} \mathfrak{C}_{i j m n}^{e}(\underbrace{\frac{\partial C_{m n}^{e}}{\partial E_{k l}}}_{\text {Eq.(C.4) }}+\underbrace{\frac{\partial C_{m n}^{e}}{\partial A_{p q}^{p}}}_{\text {Eq.(B.4) Eq.(C.9) }} \underbrace{\frac{d A_{p q}^{p}}{d E_{k l}}}_{\text {E }})$

Finalmente, o operador tangente consistente pode ser calculado pela Equação (4.95):

$\mathfrak{C}_{i j k l}^{e p}=\underbrace{\frac{d\left(A^{p}\right)_{i m}^{-1}}{d E_{k l}}}_{\text {Eq.(C.10) }} S_{m n}^{e}\left(A^{p}\right)_{j n}^{-1}+\left(A^{p}\right)_{i m}^{-1} \underbrace{\frac{d S_{m n}^{e}}{d E_{k l}}}_{\text {Eq.(C.11) }}\left(A^{p}\right)_{j n}^{-1}+\left(A^{p}\right)_{i m}^{-1} S_{m n}^{e} \underbrace{\frac{d\left(A^{p}\right)_{j n}^{-1}}{d E_{k l}}}_{\text {Eq.(C.10) }}$.

\section{C.2 Modelo visco-elástico}

Neste caso, calculam-se inicialmente as derivadas auxiliares:

$$
\begin{aligned}
\frac{\partial C_{i j}^{e}}{\partial E_{k l}} & =2\left(A^{v}\right)_{k m}^{-1}\left(A^{v}\right)_{l n}^{-1}, \\
\frac{\partial M_{i j}^{e}}{\partial E_{k l}} & =\underbrace{\frac{\partial C_{i p}^{e}}{\partial E_{k l}}}_{\text {Eq.(C.13) }} S_{p j}^{e}+\frac{1}{2} C_{i p}^{e} \mathfrak{C}_{p j m n}^{e} \underbrace{\frac{\partial C_{m n}^{e}}{\partial E_{k l}}}_{\text {Eq.(C.13) }},
\end{aligned}
$$




$$
\frac{\partial R_{i j}^{v}}{\partial E_{k l}}=-\frac{\Delta t}{\eta} \underbrace{\frac{\partial\left(M^{e}\right)_{i p}^{D}}{\partial\left(M^{e}\right)_{m n}}}_{\text {Eq.(A.22) }} \underbrace{\frac{\partial M_{m n}^{e}}{\partial E_{k l}}}_{\text {Eq.(C.14) }} A_{p j}^{v} .
$$

Usando a equação (5.33), temos:

$$
\frac{d A_{i j}^{v}}{d E_{k l}}=-(\underbrace{\frac{\partial R^{v}}{\partial A^{v}}}_{\text {Eq.(B.38) }})_{i j m n}^{-1} \underbrace{\frac{\partial R_{m n}^{v}}{\partial E_{k l}}}_{\text {Eq.(C.15) }},
$$

de onde resulta

$$
\frac{d\left(A^{v}\right)_{i j}^{-1}}{d E_{k l}}=\underbrace{\frac{d\left(A^{v}\right)_{i j}^{-1}}{d A_{m n}^{v}}}_{\text {Eq.(A.17) }} \underbrace{\frac{d A_{m n}^{v}}{d E_{k l}}}_{\text {Eq.(C.16) }}
$$

e, finalmente, pela equação (5.30):

$$
\mathfrak{C}^{v e}=\mathfrak{C}^{\infty}+\underbrace{\frac{d\left(A^{v}\right)_{i m}^{-1}}{d E_{k l}}}_{\text {Eq.(C.17) }} S_{m n}^{e}\left(A^{v}\right)_{j n}^{-1}+\left(A^{v}\right)_{i m}^{-1} \underbrace{\frac{d S_{m n}^{e}}{d E_{k l}}}_{\text {Eq.(C.11) }}\left(A^{v}\right)_{j n}^{-1}+\left(A^{v}\right)_{i m}^{-1} S_{m n}^{e} \underbrace{\frac{d\left(A^{v}\right)_{j n}^{-1}}{d E_{k l}}}_{\text {Eq.(C.17) }} .
$$

\section{C.3 Modelo visco-elasto-plástico}

Novamente, o modelo terá operadores tangentes diferentes para os casos de haver plastificação ou não. No entanto, em ambos as seguintes derivadas devem ser calculadas:

$$
\begin{aligned}
& \frac{\partial C_{i j}^{e}}{\partial E_{k l}}=2\left(A^{v}\right)_{m i}^{-1}\left(A^{p}\right)_{k m}^{-1}\left(A^{p}\right)_{l n}^{-1}\left(A^{v}\right)_{n j}^{-1}, \\
& \frac{\partial M_{i j}^{e}}{\partial E_{k l}}=\underbrace{\frac{\partial C_{i p}^{e}}{\partial E_{k l}}}_{\text {Eq.(C.19) }} S_{p j}^{e}+\frac{1}{2} C_{i p}^{e} \mathfrak{C}_{p j m n}^{e} \underbrace{\frac{\partial C_{m n}^{e}}{\partial E_{k l}}}_{\text {Eq.(C.19) }}, \\
& \frac{\partial R_{i j}^{v}}{\partial E_{k l}}=-\frac{\Delta t}{\eta} \underbrace{\frac{\partial\left(M^{e}\right)_{i p}^{D}}{\partial\left(M^{e}\right)_{m n}}}_{\text {Eq.(A.22) }} \underbrace{\frac{\partial M_{m n}^{e}}{\partial E_{k l}}}_{\text {Eq.(C.20) }} A_{p j}^{v} .
\end{aligned}
$$

\section{C.3.1 Caso não ocorra plastificação}

Neste caso, segue de imediato que

$$
\frac{d \mathbf{A}^{p}}{d \mathbf{E}}=\frac{d\left(\mathbf{A}^{p}\right)^{-1}}{d \mathbf{E}}=\mathbf{0} \quad \text { e } \quad \mathbf{D}^{\gamma}=\mathbf{0} .
$$

Logo,

$$
\begin{aligned}
& \frac{d C_{i j}^{v e}}{d E_{k l}}=2\left(A^{p}\right)_{k i}^{-1}\left(A^{p}\right)_{l j}^{-1}, \\
& \frac{d S_{i j}^{\infty}}{d E_{k l}}=\frac{1}{2} \mathfrak{C}_{i j m n}^{\infty} \underbrace{\frac{d C_{m n}^{v e}}{d E_{k l}}}_{\text {Eq.(C.23) }}=\mathfrak{C}_{i j m n}^{\infty}\left(A^{p}\right)_{k m}^{-1}\left(A^{p}\right)_{l n}^{-1},
\end{aligned}
$$


e a Equação (C.38) é simplificada para

$$
\frac{d A_{i j}^{v}}{d E_{k l}}=-(\underbrace{\frac{\partial R^{v}}{\partial A^{v}}}_{\text {Eq.(B.38) }})^{-1} \underbrace{\frac{\partial R_{m n}^{v}}{\partial E_{k l}}}_{\text {Eq.(C.21) }},
$$

permitindo calcular:

$$
\begin{aligned}
& \frac{d\left(A^{v}\right)_{i j}^{-1}}{d E_{k l}}=\underbrace{\frac{d\left(A^{v}\right)_{i j}^{-1}}{d A_{m n}^{v}}}_{\text {Eq.(A.17) }} \underbrace{\frac{d A_{m n}^{v}}{d E_{k l}}}_{\text {Eq.(C.25) }} \\
& \frac{d S_{i j}^{e}}{d E_{k l}}=\frac{1}{2} \mathfrak{C}_{i j m n}^{e}(\underbrace{\frac{\partial C_{m n}^{e}}{\partial E_{k l}}}_{\text {Eq.(C.19) }}+\underbrace{\frac{\partial C_{m n}^{e}}{\partial A_{p q}^{v}}}_{\text {Eq.(B.39) }} \underbrace{\frac{d A_{p q}^{v}}{d E_{k l}}}_{\text {Eq.(C.26) }}) \\
& \frac{d S_{i j}}{d E_{k l}}=\left(A^{p}\right)_{i m}^{-1} \mathfrak{C}_{m n p q}^{\infty}\left(A^{p}\right)_{k p}^{-1}\left(A^{p}\right)_{l q}^{-1}\left(A^{p}\right)_{j n}^{-1}+\left(A^{p}\right)_{i m}^{-1} \underbrace{\underbrace{d E_{k l}}_{d\left(A^{v}\right)_{m n}^{-1}}}_{\text {Eq.(C.26) }} S_{n p}^{e}\left(A^{v}\right)_{q p}^{-1}\left(A^{p}\right)_{j q}^{-1} \\
& +\left(A^{p}\right)_{i m}^{-1}\left(A^{v}\right)_{m n}^{-1} S_{n p}^{e} \underbrace{\frac{d\left(A^{v}\right)_{q p}^{-1}}{d E_{k l}}}_{\text {Eq.(C.26) }}\left(A^{p}\right)_{j q}^{-1}+\left(A^{p}\right)_{i m}^{-1}\left(A^{v}\right)_{m n}^{-1} \underbrace{\frac{d S_{n p}^{e}}{d E_{k l}}}_{\text {Eq.(C.27) }}\left(A^{v}\right)_{q p}^{-1}\left(A^{p}\right)_{j q}^{-1}
\end{aligned}
$$

\section{C.3.2 Caso ocorra plastificação}

Neste caso, temos:

$$
\begin{aligned}
& \frac{\partial C_{i j}^{v e}}{\partial E_{k l}}=2\left(A^{p}\right)_{k i}^{-1}\left(A^{p}\right)_{l j}^{-1}, \\
& \frac{\partial M_{i j}^{\infty}}{\partial E_{k l}}=\underbrace{\frac{\partial C_{i p}^{v e}}{\partial E_{k l}}}_{\text {Eq.(C.29) }} S_{p j}^{\infty}+\frac{1}{2} C_{i p}^{v e} \mathfrak{C}_{p j m n}^{\infty} \underbrace{\frac{\partial C_{m n}^{v e}}{\partial E_{k l}}}_{\text {Eq.(C.29) }} \\
& \frac{\partial M_{i j}^{q}}{\partial E_{k l}}=A_{m i}^{v} \underbrace{\frac{\partial M_{m n}^{e}}{\partial E_{k l}}}_{\text {Eq.(C.20) }}\left(A^{v}\right)_{j n}^{-1}, \\
& \frac{\partial \Phi}{\partial E_{i j}}=\frac{\Sigma_{k l}^{D}}{\left\|\Sigma^{D}\right\|} \underbrace{\frac{\partial \Sigma_{k l}^{D}}{\partial \Sigma_{m n}}}_{\text {Eq.(A.22) }}(\underbrace{\frac{\partial M_{m n}^{\infty}}{\partial E_{i j}}}_{\text {Eq.(C.30) }}+\underbrace{\frac{\partial M_{m n}^{q}}{\partial E_{i j}}}_{\text {Eq.(C.31) }}),
\end{aligned}
$$

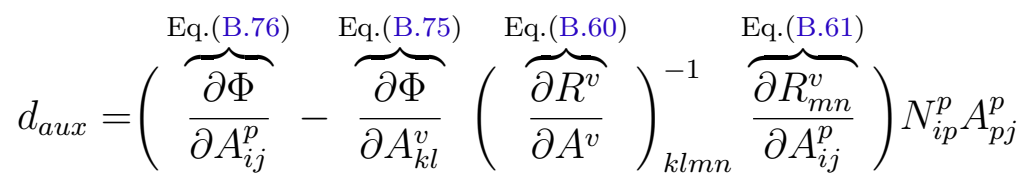

$$
\begin{aligned}
& +\underbrace{\frac{\partial \Phi}{\partial \kappa}}_{\text {Eq.(B.77) }} \sqrt{\frac{2}{3}}+\underbrace{\frac{\partial \Phi}{\partial\left(A_{i}^{p}\right)_{i j}}}_{\text {Eq.(B.78) }}\left(N_{i}^{p}\right)_{i p}\left(A_{i}^{p}\right)_{p j} \\
& D_{i j}^{\gamma}=-\frac{1}{d_{a u x}}(\underbrace{\frac{\partial \Phi}{\partial E_{i j}}}_{\text {Eq.(C.32) }}-\underbrace{\frac{\partial \Phi}{\partial A_{k l}^{v}}}_{\text {Eq.(B.75) }}(\underbrace{\frac{\partial R^{v}}{\partial A^{v}}}_{\text {Eq.(B.60) }})_{k l m n}^{-1} \underbrace{\frac{\partial R_{m n}^{v}}{\partial E_{i j}}}_{\text {Eq.(C.21) }}) .
\end{aligned}
$$




$$
\begin{aligned}
& \frac{d A_{i j}^{p}}{d E_{k l}}=N_{i m}^{p} A_{m j}^{p} D_{k l}^{\gamma}, \\
& \frac{d\left(A^{p}\right)_{i j}^{-1}}{d E_{k l}}=\underbrace{\frac{d\left(A^{p}\right)_{i j}^{-1}}{d A_{m n}^{p}}}_{\text {Eq.(A.17) }} \underbrace{\frac{d A_{m n}^{p}}{d E_{k l}}}_{\text {Eq.(C.36) }},
\end{aligned}
$$

$$
\frac{d A_{i j}^{v}}{d E_{k l}}=-(\underbrace{\frac{\partial R^{v}}{\partial A^{v}}}_{\text {Eq.(B.60) }})_{i j m n}^{-1}(\underbrace{\frac{\partial R_{m n}^{v}}{\partial E_{k l}}}_{\text {Eq.(C.21) }}+\underbrace{\frac{\partial R_{m n}^{v}}{\partial A_{p q}^{p}}}_{\text {Eq.(B.61) }} N_{p o}^{p} A_{o q}^{p} D_{k l}^{\gamma}) .
$$

$\frac{d\left(A^{v}\right)_{i j}^{-1}}{d E_{k l}}=\underbrace{\frac{d\left(A^{v}\right)_{i j}^{-1}}{d A_{m n}^{v}}}_{\text {Eq.(A.17) }} \underbrace{\frac{d A_{m n}^{v}}{d E_{k l}}}_{\text {Eq.(C.38) }}$,

$\frac{d S_{i j}^{\infty}}{d E_{k l}}=\frac{1}{2} \mathfrak{C}_{i j m n}^{\infty}(\underbrace{\frac{\partial C_{m n}^{v e}}{\partial E_{k l}}}_{\text {Eq.(C.29) }}+\underbrace{\frac{\partial C_{m n}^{v e}}{\partial A_{p q}^{p}}}_{\text {Eq.(B.41) Eq.(C.36) }} \underbrace{\frac{d A_{p q}^{p}}{d E_{k l}}}_{\text {E. }})$

$$
\frac{d S_{i j}^{e}}{d E_{k l}}=\frac{1}{2} \mathfrak{C}_{i j m n}^{e}(\underbrace{\frac{\partial C_{m n}^{e}}{\partial E_{k l}}}_{\text {Eq.(C.19) }}+\underbrace{\frac{\partial C_{m n}^{e}}{\partial A_{p q}^{p}}}_{\text {Eq.(B.43) }} \underbrace{\frac{d A_{p q}^{p}}{d E_{k l}}}_{\text {Eq.(C.36) }}+\underbrace{\frac{\partial C_{m n}^{e}}{\partial A_{p q}^{v}}}_{\text {Eq.(B.44) }} \underbrace{\frac{d A_{p q}^{v}}{d E_{k l}}}_{\text {Eq.(C.38) }})
$$

$$
\begin{aligned}
\mathfrak{C}_{i j k l}^{v e p}= & \underbrace{\frac{d\left(A^{p}\right)_{i m}^{-1}}{d E_{k l}}}_{\text {Eq.(C.37) }} S_{m n}^{\infty}\left(A^{p}\right)_{j n}^{-1}+\left(A^{p}\right)_{i m}^{-1} \underbrace{\frac{d S_{m n}^{\infty}}{d E_{k l}}}_{\text {Eq.(C.40) }}\left(A^{p}\right)_{j n}^{-1}+\left(A^{p}\right)_{i m}^{-1} S_{m n}^{\infty} \underbrace{\frac{d\left(A^{p}\right)_{j n}^{-1}}{d E_{k l}}}_{\text {Eq.(C.37) }} \\
& +\underbrace{\frac{d\left(A^{p}\right)_{i m}^{-1}}{d E_{k l}}}_{\text {Eq.(C.37) }}\left(A^{v}\right)_{m n}^{-1} S_{n p}^{e}\left(A^{v}\right)_{q p}^{-1}\left(A^{p}\right)_{j q}^{-1}+\left(A^{p}\right)_{i m}^{-1}\left(A^{v}\right)_{m n}^{-1} S_{n p}^{e}\left(A^{v}\right)_{q p}^{-1} \underbrace{\frac{d\left(A^{p}\right)_{j q}^{-1}}{d E_{k l}}}_{\text {Eq.(C.37) }} \\
& +\left(A^{p}\right)_{i m}^{-1} \underbrace{\frac{d\left(A^{v}\right)_{m n}^{-1}}{d E_{k l}}}_{\text {Eq.(C.39) }} S_{n p}^{e}\left(A^{v}\right)_{q p}^{-1}\left(A^{p}\right)_{j q}^{-1}+\left(A^{p}\right)_{i m}^{-1}\left(A^{v}\right)_{m n}^{-1} S_{n p}^{e} \underbrace{\frac{d\left(A^{v}\right)_{q p}^{-1}}{d E_{k l}}}_{\text {Eq.(C.39) }}\left(A^{p}\right)_{j q}^{-1} \\
& +\left(A^{p}\right)_{i m}^{-1}\left(A^{v}\right)_{m n}^{-1} \underbrace{\frac{d S_{n p}^{e}}{d E_{k l}}}_{\text {Eq.(C.41) }}\left(A^{v}\right)_{q p}^{-1}\left(A^{p}\right)_{j q}^{-1}
\end{aligned}
$$

